\title{
High-repetition-rate picosecond pump laser based on an Yb:YAG disk amplifier for optical parametric amplification
}

\author{
von Diplom-Ingenieur Thomas Metzger \\ aus Mutlangen
}

von der Fakultät V - Verkehrs- und Maschinensysteme der Technischen Universität Berlin zur Erlangung des akademischen Grades
Doktor der Ingenieurwissenschaften$$
\text { - Dr.-Ing. - }
$$

genehmigte Dissertation

Berlin 2009

D 83 


\section{Promotionsausschuss:}

Vorsitzender: Prof. Dr.-Ing. Henning Meyer

(TU Berlin)

1. Gutachter: Prof. Dr. rer. nat. Heinz Lehr

(TU Berlin)

2. Gutachter: Dr.-Ing. Alexander Binder

(Biotronik)

Tag der Eröffnung des Promotionsverfahrens

10.06.2009

Tag der wissenschaftlichen Aussprache:

31.07.2009 
Because I know there is consequence, it keeps me honest, it keeps me scared. Because I know I can't get away with slacking off, that the thing which happens after mediocrity is the ground. It's a game. It's a test. It is the way I live my life.

Mark F. Twight

We can't trust people who have such bad food.

Jacques Chirac 


\section{Contents}

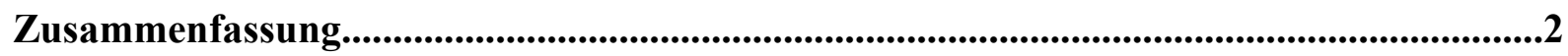

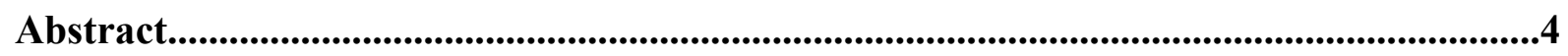

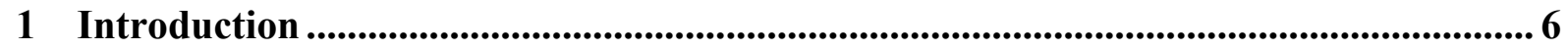

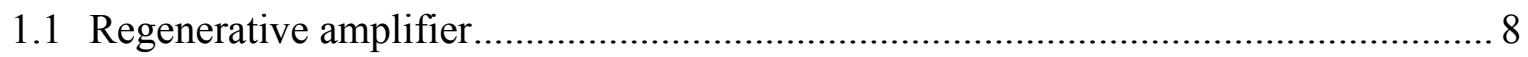

1.2 Optical parametric amplifier and high harmonic generation ................................ 10

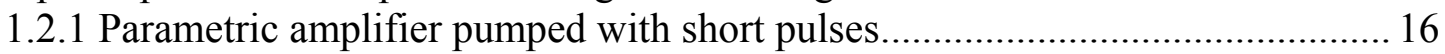

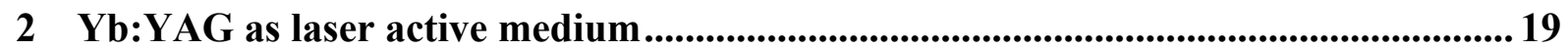

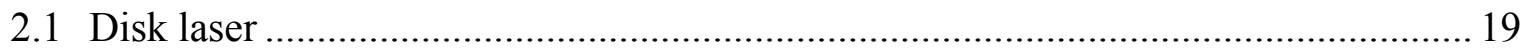

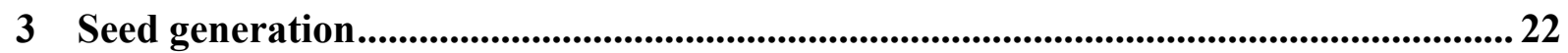

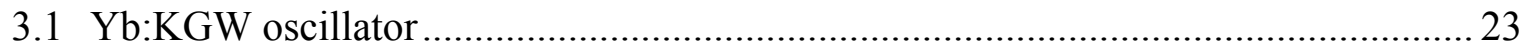

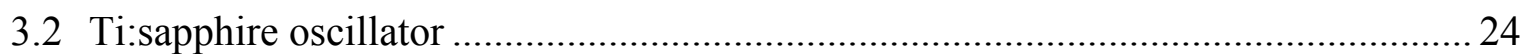

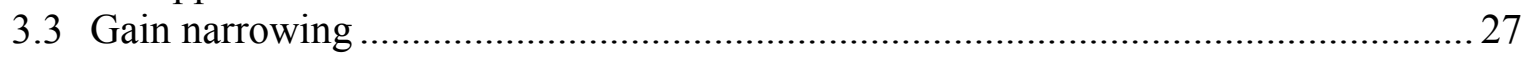

4 Preliminary considerations about the regenerative amplifier ...................................29

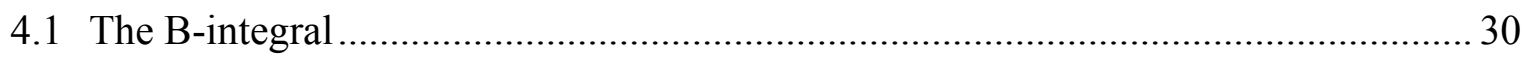

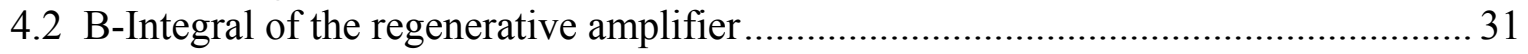

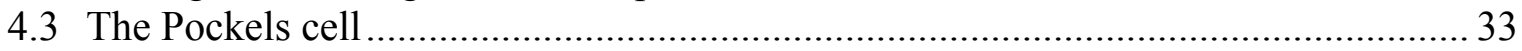

5 Regenerative amplifier without chirped pulses based on the IFSW disk laser head.36

6 Chirped pulse amplification .........................................................................................44

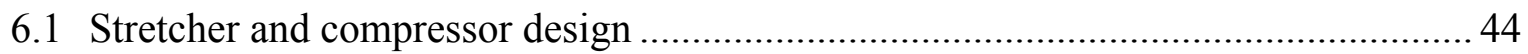

6.2 Results from the IFSW disk based regenerative amplifier with stretched seed

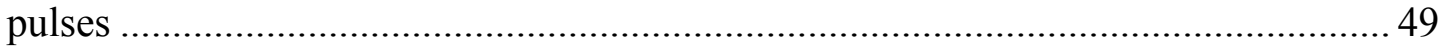

6.3 Results from the Trumpf Laser GmbH disk based regenerative chirped pulse

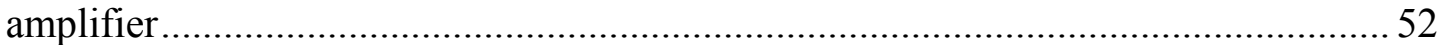

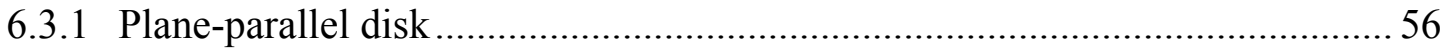

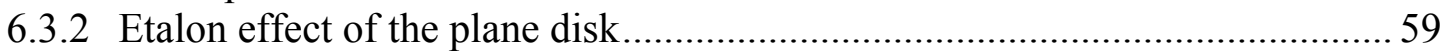

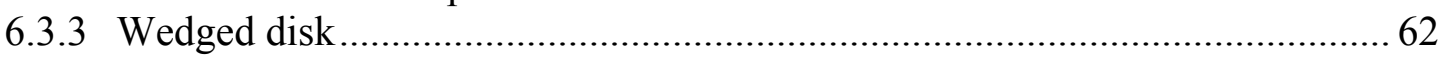

7 Regenerative amplifier based on the Trumpf Laser GmbH head with long stretcher and fiber preamplifier .........................................................................................68

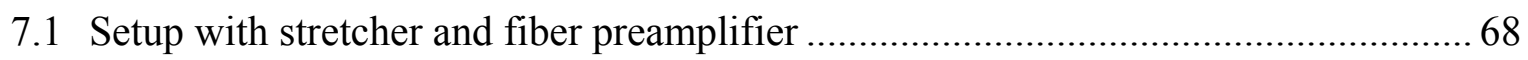

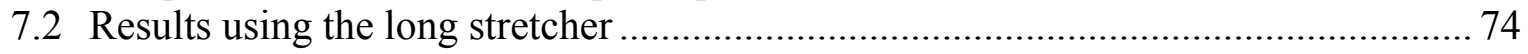

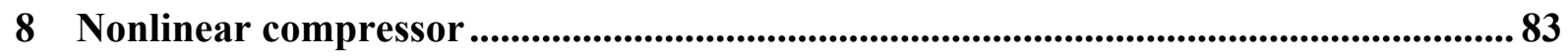

9 Multipass booster amplifier................................................................................................ 86

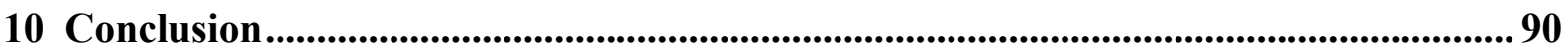

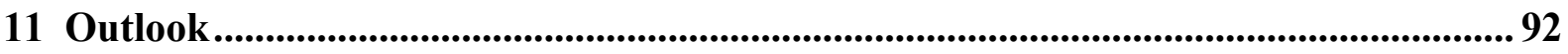

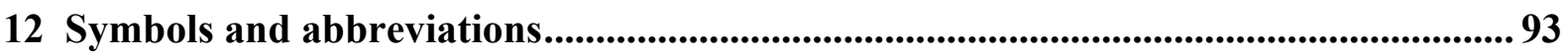

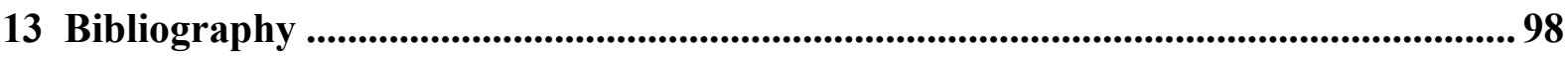




\section{Zusammenfassung}

Intensive ultrakurze Lichtpulse, vorzugsweise mit Pulswiederholraten im Kilohertzbereich, mit wenigen optischen Zyklen und vollständig kontrolliertem elektromagnetischen Feld, sind notwendig, um mittels Erzeugung von Harmonischen höherer Ordnung einzelne Attosekundenpulse zu produzieren, die auf atomarer Ebene Echtzeitbeobachtung, Manipulation und Aufzeichnung von Elektronenbewegungen ermöglichen. Seit einigen Jahren werden Pulse mit wenigen optischen Zyklen routinemäßig in Titan:Saphir-Lasern erzeugt. Ultrakurze Pulse im Bereich weniger Femtosekunden mit Kilohertzwiederholraten wurden unter Verwendung eines komplexen Aufbaus bestehend aus einem fs-Oszillator, einem Multipassverstärker für gechirpte Pulse und einer zusätzlichen, nichtlinearen Pulskompressionsstufe, demonstriert. Allerdings hat es sich als sehr schwierig erwiesen, diese Systeme in den Bereich mehrerer Millijoule Pulsenergie zu erweitern. Optische, parametrische Verstärker für gechirpte Puls haben sich als leistungsstarke Alternative erwiesen, um breitbandige Pulse mit wenigen optischen Zyklen zu generieren. Sie sind zurzeit die einzige Methode, mit der hochenergetische, kohärente Lichtpulse weniger optischer Zyklen im vielfachen Milli-Joulebereich hergestellt werden können.

Diese Dissertation konzentriert sich auf die Entwicklung eines Verstärkersystems, das speziell für das Pumpen eines effizienten, hochverstärkenden, optischen parametrischen Verstärkers mit vereinfachtem Pulsstrecker-Kompressor-Aufbau und hohem Pulskontrast konzipiert wurde. Nachfolgend ist die Entwicklung eines regenerativen Verstärkers auf Basis von dünnen Yb:YAG-Scheiben als Verstärkungsmedium beschrieben, die in verschiedenen Schritten vom einfachen Verstärker bis hin zu einem kompletten gechirpten Pulsverstärker mit Pulsspitzenleistungen von über $15 \mathrm{GW}$ durchgeführt wurde.

Die unten zusammengefasste Arbeit enthält einen einleitenden Teil, der kurz die Prinzipien der optischen parametrischen Verstärker und der Erzeugung Harmonischer höherer Ordnung beschreibt, gefolgt von Vorüberlegungen zur Seedgenerierung und nichtlinearen Effekten in Pumplasern für parametrische Verstärker. Anschließend sind die verschiedenen Schritte und Ergebnisse der einzelnen Entwicklungsstufen des regenerativen Verstärkers aufgeführt.

Kapitel 1.1 stellt das regenerative Verstärkungsprinzip dar, zusammen mit einigen wichtigen Aspekten, die bei einer regenerativen Pulsverstärkung beachtet werden müssen. Eine Einführung in die optische parametrische Verstärkung und die Erzeugung von Harmonischen höherer Ordnung wird in Kapitel 1.2 gegeben.

Das ausgewählte laseraktive Medium Yb:YAG und seine Verwendung in einer Dünnschichtkonfiguration werden in Kapitel 2 behandelt, gefolgt von der Beschreibung der Seederzeugung in Kapitel 3, die sich mit der vollständigen optischen Synchronisation zwischen der Seedquelle, dem parametrischen Verstärker und dem Pumplaser beschäftigt. Ebenfalls an dieser Stelle werden die verwendeten Yb:KGW- und Titan:Saphir-Oszillatoren charakterisiert und deren Verwendung als Seedlaser für Verstärker mit injiziertem optischen Feld dargestellt. 
Prinzipielle Überlegungen betreffend der nichtlinearen Effekte, wie Selbstfokussierung, Selbstphasenmodulation und B-Integral in einem regenerativen Verstärkersystem, sind in Kapitel 4 aufgeführt. Im Anschluss wird der speziell für das regenerative Verstärkersystem entwickelte elektro-optische Schalter zur Verringerung des kummulativen B-Integrals in Kapitel 4.3 erläutert.

In den anschließenden Kapiteln 5 bis 7 sind die entwickelten regenerativen Verstärkersysteme mit den unterschiedlichen Laserköpfen vom Institut für Strahlwerkzeuge der Universität Stuttgart und der Trumpf Laser GmbH \& Co. KG aus Schramberg beschrieben und charakterisiert. Zuerst wird der Ansatz der ungechirpten Verstärkung in Kapitel 5 präsentiert, gefolgt von den Verstärkersystemen in Kapitel 6 und 7, die in zwei unterschiedlichen dispersiven Verzögerungsstufen jeweils unterschiedliche Laufzeiten für verschiedene Frequenzen einführen, so zu längeren Pulsdauern während dem Verstärkungsprozess führen und dadurch die auftretenden Pulsspitzenintensitäten deutlich reduzieren.

Ein weiterer Ansatz zur zusätzlichen Verkürzung der Pulsdauer des fertig gestellten Pikosekunden-Systems aus Kapitel 7.2 mit Hilfe einer zusätzlichen nichtlinearen Kompressorstufe ist in Kapitel 8 erläutert und durch erste Messungen charakterisiert. Ein Vorschlag zur weiteren Verstärkung der Pulse in den Bereich einiger Joule Energien bei Kilohertzwiederholraten ist in Kapitel 9 ergänzend angefügt.

Diese Dissertation wird durch die Zusammenfassung und den Ausblick in den Kapiteln 10 beziehungsweise 11 beendet.

Die im Rahmen dieser Dissertation entstandenen Veröffentlichungen sind im Anhang aufgeführt. 


\begin{abstract}
Intense ultrashort light pulses with full control of the subcycle field evolution preferably with kilohertz repetition rates are required to produce via high-order harmonic generation controlled single attosecond pulses allowing real-time observation, manipulation and tracking of atomic-scale electron dynamics. By the turn of the millennium few-cycle light pulses are routinely generated in Ti:sapphire based lasers. Ultrashort pulses in the range of a few femtoseconds at kilohertz repetition rate have been demonstrated using a complex design consisting of an oscillator, a multipass chirped pulse amplifier and an additional nonlinear compression stage. However, it has been difficult to extend these systems to the multi-mJ level. Optical parametric chirped pulse amplifiers have emerged as a powerful alternative for creating broadband few-cycle pulses, and are the only method by which high energy multi-mJ fewcycle coherent light pulses have been generated.
\end{abstract}

This thesis focuses on the development of an amplifier laser system especially designed for use in pumping an efficient, high gain, high contrast, optical parametric chirped pulse amplifier with a simplified stretcher-compressor system. Consecutively described is the development of a regenerative amplifier based on an Yb:YAG thin disk as gain medium carried out in several evolutionary steps from a master oscillator power amplifier setup to a complete chirped pulse amplifier system with peak powers above $15 \mathrm{GW}$ marking the birth of a new generation of pump lasers for optical chirped pulse amplifiers.

This work summarized below is composed of an incipient part mentioning briefly the principle of optical parametric amplifiers and the generation of high-order harmonic generation followed by preliminary considerations such as seed generation and nonlinear effects for building a parametric amplifier pump source and finally the performed steps and results of the regenerative amplifier laser development.

Chapter 1.1 introduces the regenerative amplifier, its principle and aspects to be considered regarding regenerative amplification. An introduction into optical parametric amplification and high-order harmonic generation is given in chapter 1.2.

The chosen laser active medium Yb:YAG and its use in a thin disk laser configuration are mentioned in chapter 2 followed by the seed generation section in chapter 3 describing the alloptical synchronization between the seed laser, the parametric and regenerative amplifier and the characterization of the used $\mathrm{Yb}: \mathrm{KGW}$ and Ti:sapphire seed oscillators and their use as a seed source for amplifier with injected optical fields.

Presented in chapter 4 are the preliminary considerations about the regenerative amplifier regarding the nonlinear effects such as self focusing, self phase modulation and B-integral in the amplifier system and the especially designed electro-optical switch meeting the requirement of a low cumulative B-integral for a high peak power laser.

In the following three chapters 5 to 7 the developed regenerative amplifier systems using amplifier heads from the Institut für Strahlwerkzeuge at the University Stuttgart and Trumpf Laser GmbH \& Co. KG from Schramberg are pronounced and characterized. First mentioned is the non chirped pulse amplification approach in chapter 5 pursued by the amplifier systems 
seeded with temporally stretched seed pulses using two different dispersive delay lines introducing different velocities or path lengths to different frequency components of the pulses leading to much longer pulse durations with reduced peak intensities in chapter 6 and 7 respectively.

A further approach to shorten the pulse duration of the completed laser system characterized in chapter 7.2 is demonstrated in chapter 8 using a nonlinear compression scheme. To further boost the pulse energy into the multi-joule and multi-kilohertz regime a proposal is given in chapter 9 .

This work is finalized by the conclusion in chapter 10 and the outlook in chapter 11 .

The publications emerged in the framework of this thesis are listed in the addendum. 


\section{Introduction}

Light surrounds us everywhere and information transferred via light constitutes the main part of our perception. Since the first demonstration of a laser some 40 years ago [1], these sources of coherent light play an important role in science. Based on laser sources, a plurality of effects in atomic and molecular physics has been studied and light-matter interaction in general is a fast growing field with many important implications and applications. The discovery of the photoelectric effect by Einstein opened the field of research that deals with the interplay of matter and light.

After the advent of mode-locked laser sources 1964 [2,3], the attainable pulse duration decreased and approaches nowadays the ultimate limit of a single field oscillation, which corresponds to $\sim 2.5$ femtoseconds $\left(10^{-15} \mathrm{~s}\right)$ for visible light [4]. During this development, the advances in optical technology opened more and more fields in not only atomic, but also molecular and solid-state physics and, awarded with the Nobel prize to Zewail in 1999, realtime chemistry. Ultrashort laser pulses not only serve scientists as a fast shutter in terms of the sharp temporal confinement, but also feature another characteristic making them interesting for science: they offer unprecedented intensity. Focused short pulse lasers provide electric fields that rival the attractive electrostatic potential that binds electrons to nuclei, and are at the same time fully controllable making them an ideal tool for steering and controlling charge in atoms and molecules [5].

The exchange of elementary charges between atomic systems constitutes the basic process of any chemical reaction. The question, how molecules and finally the world surrounding us is formed based on this charges transfer, is a driving force since the beginning of science. Due to the enormous speed that characterizes those processes, the methods to investigate them so far where restricted to comparing the initial and the final state of the system and had to guess the interim evolution. For many intra-atomic processes, femtosecond temporal resolution is not yet satisfactory. Attosecond science holds the promise to push the available temporal resolution by at least an order of magnitude and allows extending conventional ultrafast spectroscopy and strong-field coherent control from the cycle-averaged to the sub-cycle domain of visible light [6]. Attosecond technology is based on the nonlinear response of atoms exposed to ultra-intense laser fields and is usually described as the absorption of many low-energetic (laser) photons followed by the emission of a single high-energetic photon.

Employing short-pulse lasers for such studies is advantageous for two reasons: First of all, the interaction of charged particles with electric fields is well understood, facilitating the analysis and interpretation of the acquired data. Secondly, modern short-pulse laser sources emit pulses that merely comprise 1.5 field cycles of visible light [7]. They are therefore sources for the routine generation of the shortest controllable signals and thus ideal tools for the investigation of ultrafast processes. Based on the frequency conversion of high-power laser pulses by high-order harmonic generation $(\mathrm{HHG})[8,9]$, the spectral extent of the light is shifted into the extreme ultraviolet (XUV). This shift is accompanied by an additional shortening of the pulse duration and enables a series of experiments regarding the charge transfer in matter. 
Producing such isolated attosecond XUV pulses via HHG requires high-energy, few cycle pulses preferably with kilohertz repetition rates for exploiting attosecond physics $[4,10,11]$. Ultrashort pulses in the range of a few femtoseconds at kilohertz repetition rate have been demonstrated using a complex design consisting of an oscillator, a multipass chirped pulse amplifier and an additional nonlinear compression stage $[12,13]$. However, it has been difficult to extend these systems to the multi-mJ level [14,15] but, nevertheless, due to the low conversion efficiency from the driving source to the produced X-ray photons especially of an infrared laser driven HHG, the driver source must have high pulse energy and pulse durations as short as possible preferably of a single optical cycle.

Optical parametric chirped pulse amplifiers (OPCPA) have emerged as a powerful alternative for creating broadband few-cycle pulses, and are the only method by which high energy mulit-mJ few-cycle coherent light pulses have been generated [16,17,18]. However, current OPCPA designs suffer from complex stretcher and compressor elements, necessitated by the pulse duration of pump laser pulses, which are currently several tens of picoseconds in duration or longer [19,20,21]. Stretching the seed pulse to a significant fraction of the pump pulse duration is required for efficient energy extraction, but extensive stretching to tens of picoseconds requires highly dispersive prisms or grating components and subsequently intricate adaptive dispersion management schemes for proper recompression [21,22]. The use of shorter pump pulses in the range of a few picoseconds [23] would eliminate the need for such a large stretching and compression ratio. In this case, for efficient energy extraction, the seed pulse can be stretched using only a few chirped mirrors [24], and recompressed in a highly efficient compressor consisting of only a few centimeter of bulk dispersive material. Furthermore, the damage threshold for nonlinear crystals decreases at a significantly lower rate than the longpulse $\tau^{1 / 2}$ scaling for incident pulse durations above $20 \mathrm{ps}$ [25], allowing more efficient energy conversion to the signal for OPCPAs pumped by shorter pulses, as the parametric gain is intensity dependent. As a consequence of higher pump intensities, the supported bandwidth in an OPA can be drastically increased due to simplified phase matching conditions by using shorter nonlinear crystals [26].

Recent experiments in the area of infrared (IR) multi-kilohertz few ps-amplifiers without chirped pulse amplification (CPA) [27] showed temporal pulse broadening due to self phase modulation (SPM) in the electro-optic modulator for energies below $1 \mathrm{~mJ}$ [28, 29]. While in principle, scaling of the Pockels cell (PC) dimensions allows for larger beam diameters and, hence, pulse energies at equal average output power, this scaling also requires increasingly inconvenient high voltages and larger required crystals that become difficult to fabricate. Since the SPM-induced spectral broadening at a given pulse energy is proportional to the square of the pulse duration, detrimental non-linear effects can be overcome utilizing CPA. Fortunately, at wavelengths around $1 \mu \mathrm{m}$ stretchers and compressors of insignificant loss are available utilizing transmission or reflection gratings with efficiencies as high as $97 \%$ and $99 \%$ respectively [30,31].

Consecutively described is the development of a regenerative amplifier based on an Yb:YAG thin disk as gain medium carried out in several evolutionary steps from a master oscillator power amplifier setup to a complete chirped pulse amplifier system with peak powers above 
$15 \mathrm{GW}$. To characterize the systems the following devices were used: A power meter head from Coherent Inc. LM-100 HTD together with the Fieldmaster read out. The pulse energy was determined via average power and repetition rate. Spectral measurements were done with a spectrometer HR4000 for a wavelength range from 950 to $1100 \mathrm{~nm}$ and a resolution of $0.05 \mathrm{~nm}$ from Ocean Optics Inc. together with the SpectraSuite Spectroscopy Operating Software. Pulse duration measurements were done with a home made second harmonic generation intensity autocorrelator [32] with a total scanning range of approximately $600 \mathrm{ps}$ and a dynamic range of approximately $10^{4}$.

\subsection{Regenerative amplifier}

Short optical pulses with high peak power have been generated by mode locked oscillators $[33,34]$ with pulse energies between a few $n J$ with durations as short as 6 fs [35] and up to $>25 \mu \mathrm{J}$ with pulse durations of nearly 1 ps [36]. To reach significant higher pulse energies while keeping the pulse duration short amplifier or amplifier chains are necessary. Regenerative amplifiers are commonly used to strongly amplify selected individual optical pulses from a train of low-energy pulses emitted by mode-locked oscillators and allow many passes through an inverted gain medium placed in an optical resonator. An optical switch such as a Pockels Cell (PC) injects the selected pulses into the cavity, controls the number of round trips and ejects the amplified pulse usually when the gain is saturated. The pulse width is typically given by the source of initial pulses and its final amplitude is determined by the amplifier's gain and loss.

\section{regenerative amplifier}

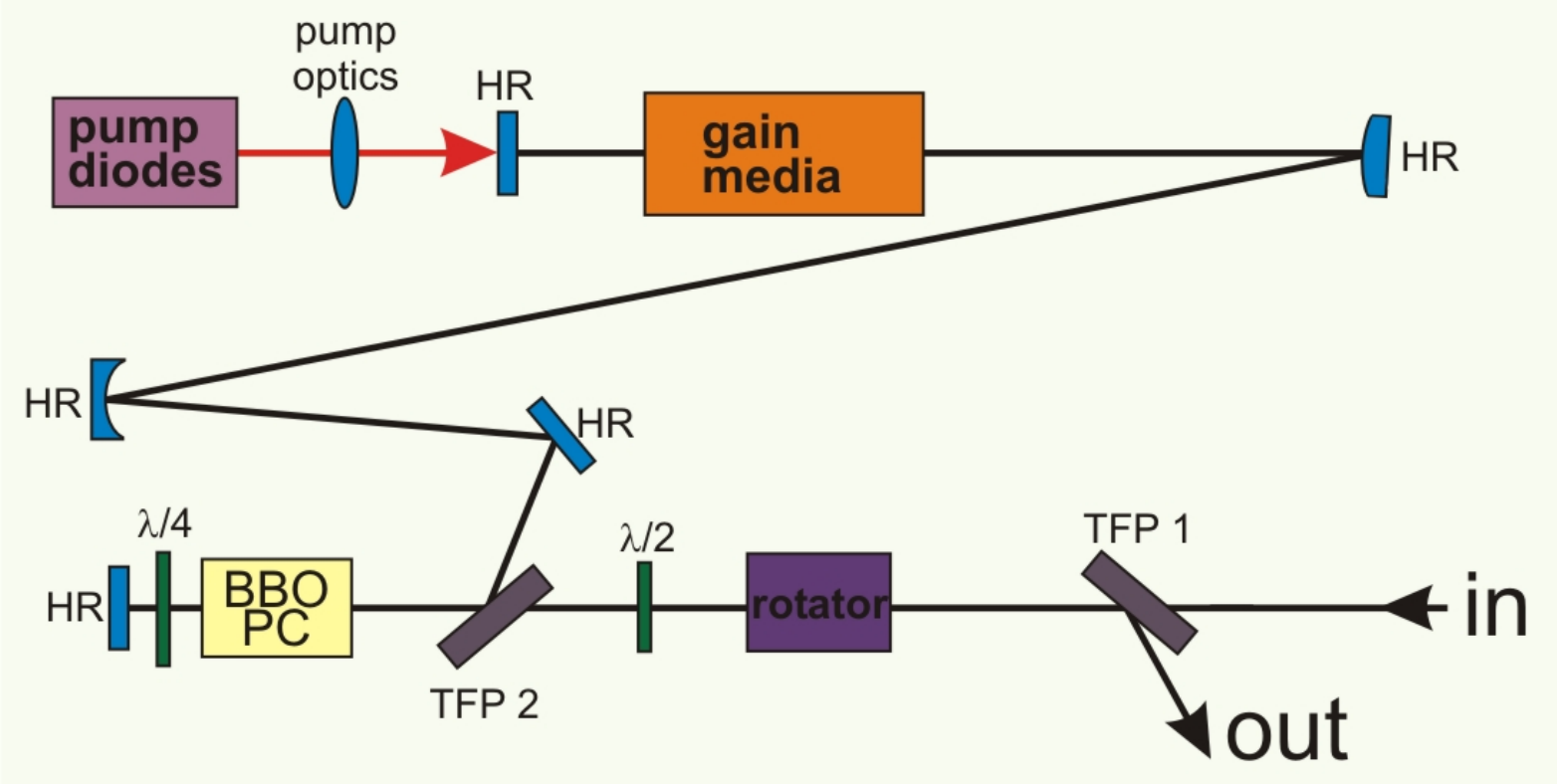

Fig. 1-1 Setup of a regenerative amplifier. HR, high reflecting mirror; TFP, thin film polarizer; $\lambda / 2$, half wave plate; $\lambda / 4$ quarter-wave plate.

Figure 1-1 shows the setup of a typical regenerative amplifier. The functionality is shortly described below. A more detailed description is given in [37]. In operation, the horizontally 
polarized incoming pulse passes through an optical isolator build by the two thin film polarizers (TFP) and the Faraday rotator, enters still horizontally polarized the cavity sector containing the PC and the intracavity quarter-wave plate $(\lambda / 4)$, hits the high reflecting mirror (HR) at the end of the cavity, is reflected back traveling a second time through the quarter-wave plate and is reflected via TFP 2 into the cavity due to the 90 degree polarization rotation by the double pass trough the quarter-wave plate. In this initial state, the PC is still switched off and has no influence on the polarization and the now vertical polarized pulse experiences a reflection at TFP 2 into the cavity. While the pulse is traveling towards the gain media a voltage is applied to the PC to compensate for the polarization rotation introduced by the intracavity quarter-wave plate keeping the pulse trapped inside the cavity as the polarization is now kept vertically. Once trapped inside the cavity as long as the voltage is applied to the PC the vertical polarized pulse is amplified while passing twice per roundtrip through the inverted gain media. After the amplification cycle containing often more than 100 round trips inside the resonator the pulse is ejected from the cavity via switching off the quarter-wave voltage of the PC. After switching off the PC the amplified pulse polarization is rotated again by 90 degrees through passing twice trough the intracavity quarter-wave-plate allowing the horizontally polarized pulse exiting the cavity via TFP 2. Finally the Faraday rotator and TFP 2 separate the incoming pulse train from the amplified pulse.

While no pulse is being amplified in the cavity, continuous wave (cw) laser action is prevented by the quarter-wave plate and the TFP 2 acting together as a $100 \%$ output coupler. After the first solid state regenerative amplifier in 1980 [38], regenerative amplifiers with repetition rates typically between a few $\mathrm{kHz}$ and several $100 \mathrm{kHz}$ and pulse energies in the range of a few $\mathrm{mJ}$ and $\mu \mathrm{J}$ respectively could be demonstrated [39-42].

The following aspects have to be considered regarding regenerative amplification:

- The usually limited bandwidth of the amplifier gain medium can increase the duration for an unchirped pulse via gain narrowing. In case of amplifying a chirped pulse so called pulse steepening leads to shorter amplified pulse durations. Both effects are elucidated in chapter 3 .

- Introduced by the optical intracavity components (PC, gain media, quarter-wave plate) dispersion can increase the pulse duration of the amplified pulse. In some special cases the positive dispersion can also be used to compress a previously negative chirped pulse [40].

- To keep the efficiency and the overall gain of the amplifier high, round trip losses inside the resonator should be kept as small as possible. The influence of gain, gain recovery due to pumping, losses, input and output energy are described in $[38,43]$.

- Nonlinear effects caused by bulk material inside the amplifier cavity as the PC and the gain media can lead to temporal pulse distortion due to self phase modulation (SPM) and sever optical damage by self focusing (SF). SPM and SF are described in chapter 4.1. 


\subsection{Optical parametric amplifier and high harmonic generation}

The regenerative amplifier presented in this work has been designed for creating high energy pulses for use in pumping an efficient, high gain, high contrast, infrared OPCPA with a simplified stretcher-compressor system designated as a driving source for high harmonic generation. Therefore a short review about OPCPAs and HHG is given at this point to emphasize the need of such a picosecond pump source.

Parametric amplification is a nonlinear process of second order used for converting a pump wave into signal photons of lower energies. A signal with the frequency $\omega_{1}$ is amplified in a nonlinear crystal in the presence of an intense so-called pump field of the frequency $\omega_{3}\left(\omega_{3}>\right.$ $\left.\omega_{1}\right)$. The pump wave is converted into the signal photon of lower energy $\omega_{1}$ and following the energy conservation the same numbers of so-called idler photons of the frequency $\omega_{2}=\omega_{3}$ $\omega_{1}$ are produced. The process is called parametric due to the modulation of the system parameter $\chi$, the nonlinear optical susceptibility, in consequence of the pump field $\omega_{3}$ [44]. One of the big advantages of the parametric amplification is the full conversion of the pump signal into the energy of the signal and idler beam and therefore the nonlinear crystal is not heated and thermal effects are totally absent.

Parametric amplification was predicted in the early 1960s and the concept was soon applied in optical parametric oscillators (OPO) $[45,46]$ and further proved to be a convenient way to extend the tunability of femtosecond lasers $[47,48]$ based on noncollinear parametric converters. In 1992, the first OPA based on chirped pulse amplification techniques (OPCPA) was realized [49] before in 1986, Piskarskas et al. [50] investigated phase phenomena in parametric amplifiers in a similar system. A substantial contribution to the success of parametric amplification was made by the discovery of new nonlinear optical crystals with nonlinear optical properties not seen before like BBO and LBO [51]. In 1997, Ross et al. [52] proposed the use of OPCPA-techniques to amplify laser pulses to Petawatt peak power levels with a "table-top" amplifier. Since then, remarkable progress has been made in the development of OPCPA for example to achieve high gain, with a $37 \%$ high conversion efficiency and high contrast a hybrid chirped-pulse amplification scheme that uses a single pump pulse and combines optical parametric amplification and laser amplification had been developed [53]. In 2007, Tavella et al. reached the 10 Terawatt regime with an OPCPA delivering few-cycle pulses with pulse durations below $10 \mathrm{fs}$. The progress of OPA/OPCPA development is summarized in the references [26,54]. The advantages, challenges and disadvantages of OPAs are hereinafter referred to as:

\section{Advantages:}

- Gain bandwidth: One of the most remarkable properties of parametric amplification is the possibility of large bandwidth amplification without substantial gain narrowing depending on geometry of interaction, crystal type, crystal thickness and wave vector phase-matching achieved mostly through noncollinear optical parametric interaction. 
- Single-pass gain: The single pass gain achievable in an OPCPA is extremely high. It can reach up to six orders of magnitude.

- Thermal load: The thermal load in an OPCPA is negligible till highest pulse repetition rates. The energy is not stored in the amplifier material as it is in a solid state gain medium, but the energy conversion from pump to idler and signal is an instantaneous parametric effect.

- Efficiency: High efficiencies can be reached with OPCPAs. Typical reached efficiencies are in the range of $20-25 \%$ conversion from the pump laser to the signal wavelength.

- Scalability to high energies: OPCPA can be scaled to reach even higher peak powers than with conventional CPA-amplifiers by reducing the pulse duration of the amplified signal pulses. The final compressed signal pulse duration can be much shorter due to the large amplification bandwidth.

- Versatility to achieve phase matching: There are several degrees of freedom that can be exploited to achieve broadband phase matching. Among them, and most important, the non-collinear angle between pump and signal, but also the pump beam bandwidth (chirped pump), multiple beam pumping with different noncollinear angles, or an angularly chirped signal or pump can be used.

- Idler wave: The idler wave can also be useful in some case for example to generate a seed beam in spectral regions difficult to reach with conventional techniques or to use the idler wave to stabilize the carrier envelope offset $[55,56]$.

\section{Challenges:}

- Pump-to-signal synchronization: Amplification takes only place in the nonlinear crystal when pump and signal are present at the same time. Therefore precisely synchronization in time either by electronic or optical synchronization is required.

- Stretching and compression: Stretching the seed pulse to a fraction of the pump pulse duration is required to extract energy out of the pump laser. In case of long pump pulses the recompression over four orders of magnitude of the amplified signal in the ps- or even ns-range to a few-cycle pulse is difficult to achieve.

- Strict phase matching conditions: Alignment of a parametric amplifier involves several issues including geometry of interaction, pointing stability, pump energy fluctuations, beam collimation, etc. and must all be considered in order to obtain reliable operation.

\section{Disadvantages:}

- Background emission \& temporal contrast: Background emission of an OPCPA has an entirely different mechanism of generation compared to amplified spontaneous emission (ASE) in a solid-state laser amplifier. The background emission in 
an OPA is created by optical parametric superfluorescence due to spontaneous decay of a pump photon into an idler and a signal photon which is further parametrically amplified.

- Pump beam requirements: The requirements for the pump in an OPCPA are demanding. The pump should deliver highest possible energy at a good beam quality. The amplified signal spatial beam profile is directly related to the optical properties of the pump beam. Even small imperfections in the pump beam profile will appear enhanced in the signal beam profile due to the high parametric gain.

- Energy stability: Near saturation, the OPCPA output can reach only a pulse to pulse stability close to the pump pulse energy stability.

- Spatial chirp: The non-collinear parametric amplification process is a directional process. If a poor choice of interaction geometry is made, spatial chirp and spatial dispersion of the amplified beam occur.

A further advantage of OPCPAs is the development of few-cycle pulses at different wavelengths than provided by Ti:sapphire based lasers. Isolated attosecond pulses of photon energies up to $\sim 100 \mathrm{eV}(13 \mathrm{~nm})$ can now be routinely generated and utilized in a host of interesting applications requiring atomic time resolution, creating a new entire field of attosecond physics [57]. Typically, such pulses are generated by high harmonic generation (HHG) in a noble gas, driven by intense few-cycle laser pulses provided by a Ti:sapphire laser amplifier system at a wavelength around $800 \mathrm{~nm}[58,59]$.

To understand reaction dynamics on atomic or molecular levels it is necessary to observe the inner electron shells. X-ray diffraction on the time base of an atomic unit of 24 as would allow 4-dimentional imaging of the electronic structure of matter with atomic resolution not only in space but also in time as shown in figure 1-1. The ultrashort electron or x-ray pulse allows recording snapshots of the dynamic evolution of the electron density distribution following excitation by a short pump pulse. Dynamic changes in the electron distribution may occur due to first the motion of the nuclei; the electron cloud virtually instantly adjusts to this motion; or second electronic excitation. The former process evolves on a multi-fs time scale and mirrors atomic rearrangement in molecules or solids. Electronic rearrangements may unfold within attoseconds [6]. A further application of an infrared driven HHG results from the created short wavelength in the so-called water window between 2.3 and $4.4 \mathrm{~nm}$. Within the water window carbon atoms strongly absorb while water is transparent. Therefore submicroscopic analysis of tissue is possible allowing new perspectives in the fields of biophysics, pharmaceutical research and chemical synthesis. Low-order nonlinear frequency conversion processes such as second-harmonic generation, i.e. wave-mixing of the fundamental with the nonlinear dipole response of the interacting medium, leads to the emission of frequency shifted light already at comparably low laser intensities. In contrast to that, high order harmonics result from the highly nonlinear interaction of ultra-intense laser pulses with typically a gaseous target medium [60]. Figure 1-2 shows an X-ray beam created by focusing a Ti:sapphire laser with $5 \mathrm{fs}$ short pulses and $1.5 \mathrm{~mJ}$ pulse energy into helium gas [9]. 


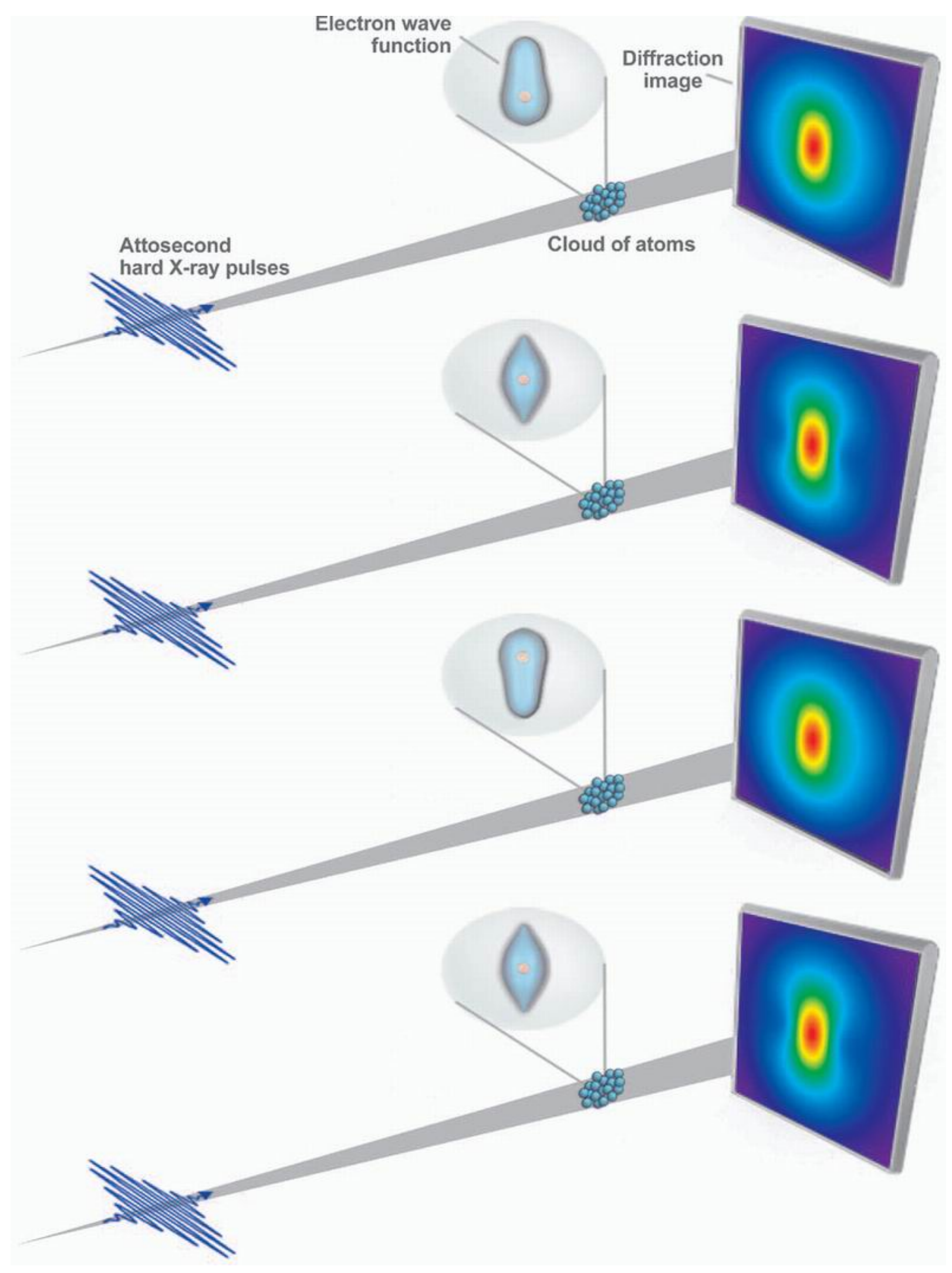

Fig. 1-1 Schematic of attosecond diffraction imaging of dynamic changes of atomic-scale electron distribution. As an example, hydrogen atoms excited into the 1S-2P coherent superposition state have been exposed to 100-as, 1- $\mathrm{\AA}$ x-ray pulses in a numerical experiment. From the recorded freeze-frame diffraction images shown on the screens, the instantaneous electron density distribution can be determined. From a series of such images electronic motion can be reconstructed with attosecond resolution in time and picometer resolution in space. Courtesy of $\mathrm{V}$. Yakovlev. 


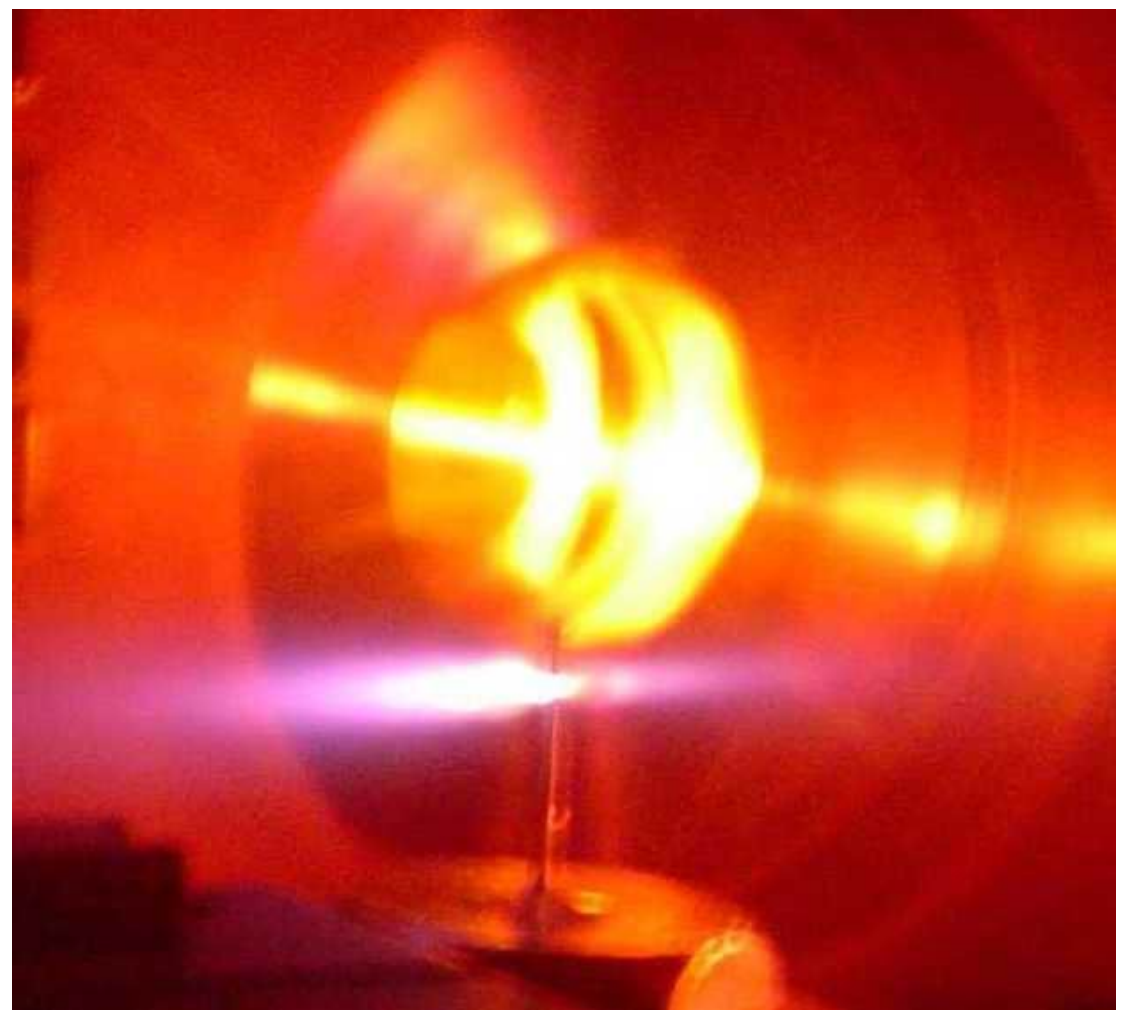

Fig. 1-2 Laser driven X-ray source in the water window (2.3-4.4 nm) produced in Helium with the following pump pulse parameters: $5 \mathrm{fs} ; 1.5 \mathrm{~mJ}, 750 \mathrm{~nm}, 1.0 \mathrm{kHz}$.

The basic generation mechanism for higher-order harmonics is successfully described by the "three-step-model" introduced by Corkum and Kulander [61] and summarized in figure 1-3. The three steps including tunnel ionization, the motion of the electron inside the laser electric field and the subsequent recombination with the parent ion are described below and in the figure caption.

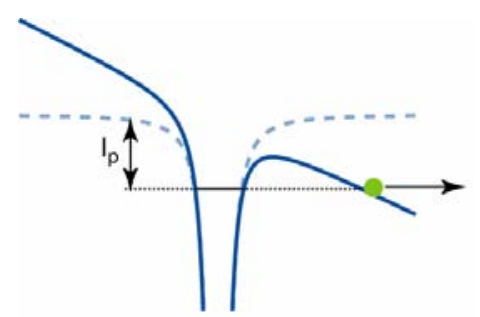

tunnel ionization

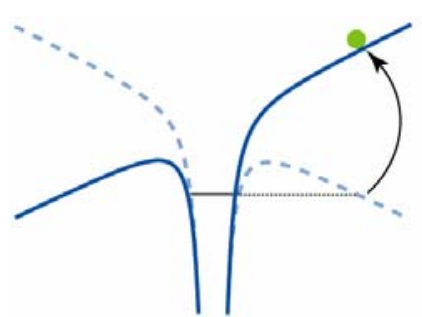

acceleration in the laser field

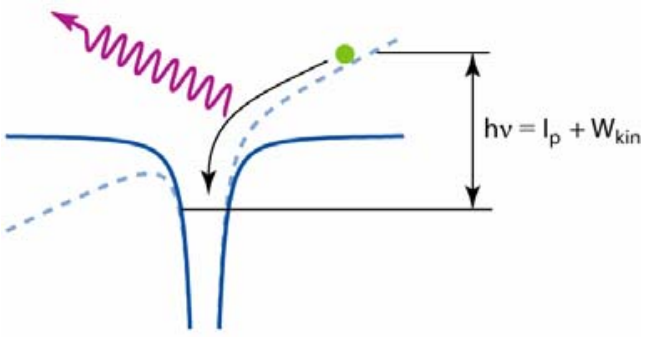

recombination and photoemission

Fig. 1-3 Illustration of the three-step-model for high-harmonic radiation. First (left side) the laser electric field releases the electron from the atomic attractive potential via tunnel ionization $\left(I_{p}\right.$ : binding energy of the electron). Then (center panel) changing its sign after half a laser period, the electric field accelerates the electron first away from the ion and then back. The right panel shows the recombination of the electron with the ion and the emission of a high-energy photon. The attainable energy of the emitted photon depends on the ionization potential and the kinetic energy $\left(W_{\text {kin }}\right)$ the electron gained during its acceleration in the laser electric field.

Higher laser intensities cause a dramatic distortion of the atomic potential such that a potential barrier is formed featuring a local maximum called the saddle point (figure 1.3 left side). If the laser field frequency is low enough, the electron can escape from the atom by tunneling 
through the potential barrier. The electron is set free via tunnel ionization due to the intense field by the optical few-cycle pulse with zero initial velocity and accelerated away from its parent ion by the laser electric field. It moves along a classical electron trajectory in the laser field and as soon as the electric field reverses, the electron is first decelerated and then accelerated back towards its parent ion. Recombination of the electron with its parent ion leads to the emission of a potentially extreme-ultraviolet (XUV) photon. The maximum cutoff energy $\hbar \omega$ in the high-harmonic spectrum is given in atomic units and can be calculated using classical [62] or quantum mechanics [63]. It is given by (the more accurate quantum mechanical treatment introduces a factor of $\sim 1.3$ to the ionization potential)

$$
(\hbar \omega)_{\max }=\mathrm{I}_{\mathrm{p}}+3.17 \mathrm{U}_{\mathrm{p}}
$$

where $\mathrm{I}_{\mathrm{p}}$ is the atom's ionization potential, and

$$
U_{p}=\frac{e^{2} E^{2}}{4 m \omega^{2}}
$$

is the ponderomotive potential and expresses the cycle averaged kinetic energy of a free electron oscillating in an electric field of intensity I, where E is the driver laser's field strength, e the charge and $m$ the mass of the electron and $\omega$ is the frequency [64]. It has been suggested and recently demonstrated [65] that using a long-wavelength driver, coherent $\mathrm{x}$-ray photon energy cutoff can be significantly extended. Equation 1-2 shows that the maximum cutoff energy scales with the quadratic driver wavelength. The wavelength-scaling law of the HHG yield remains an interest of theoretical investigation [66,67]. In 2006 a $1 \mathrm{kHz}$ carrierenvelope-phase (CEP) stable 20-fs 2.1 - $\mu$ m-carrier-wavelength system, generating $\sim 80 \mu \mathrm{J}$ pulses with a well-suppressed parametric superfluorescence background, pumped by a Nd:YLF laser system, using the concept of OPCPA was presented [68] and later in 2009 further developed to higher pulse energies of $740 \mu \mathrm{J}$ [19]. Such a system with higher output energy in the mJ-range will be an ideal driver for HHG of coherent x-ray photons in the keV regime and could for the first time produce single attosecond pulses of $<30$ as in a wavelength regime of $1 \mathrm{~nm}(1 \mathrm{keV})$ and would allow observations of inner electron shells with their faster dynamics.

One photon per electron is emitted carrying the sum of the ionization potential and the electron's kinetic energy. The recombination event itself is rather unlikely, the conversion efficiency therefore low $\left(\sim 10^{-6}\right.$ in the cut-off range). From the definition of the ponderomotive potential above and the estimation for the highest reachable recombination energies it is apparent, that both the laser frequency and intensity determine the cut-off energy. Concluding that a longer driving laser wavelength at the same peak intensity triggers the emission of higher energetic photons led to several attempts to confirm this experimentally [69-72]. Newer experimental results see the generation of high-harmonic-radiation with short driving wavelengths $\lambda$ in favor again $[73,74]$. As a rule of thumb, the efficiency scales as $1 / \lambda^{5}$, while the cut-off grows according to $\lambda^{2}$. The high photon energies that can be achieved in this process together with the preserved temporal confinement of the emission make high-harmonic generation the ideal source for ultrashort high-energetic XUV bursts. However, the large ex- 
cursion the electron undergoes to accumulate kinetic energy (exceeding the fundamental photon energy several times) leads to a substantial spread of the free electron wavepacket reducing the recombination probability drastically. This wavepacket dispersion is estimated by $[75,76]$ and justifies the little reabsorption probability of the electron as devoted to its quantum nature.

Due to the low conversion efficiency from the driving source to the produced X-ray photons especially of an infrared laser driven HHG, the driver source must have high pulse energy and pulse durations as short as possible preferably of a single optical cycle. The laser developed during this work was especially tailored to meet the requirements of a pump laser used for a few-cycle infrared driver source for HHG based on the concept of an IR-OPCPA mentioned above $[19,68]$.

\subsubsection{Parametric amplifier pumped with short pulses}

Optical parametric chirped pulse amplifiers (OPCPA) have emerged as a powerful alternative for creating broadband few-cycle pulses, and are the only method by which high energy multi-mJ few-cycle coherent light pulses have been generated [16-18]. However, current OPCPA designs suffer from complex stretcher and compressor elements, necessitated by the pulse duration of pump laser pulses, which are currently several tens of picoseconds in duration or longer $[19,20,34]$. Stretching the seed pulse to a significant fraction of the pump pulse duration is required for efficient energy extraction, but extensive stretching to tens of picoseconds introduces high losses to the seed energy and requires highly dispersive prisms or grating components and subsequently intricate adaptive dispersion management schemes for proper recompression [21,22]. The use of shorter pump pulses in the range of a few picoseconds [23] would eliminate the need for such a large stretching and compression ratio. In this case, the seed pulse can be stretched by passing it through a few-centimeter-long dispersive optical material and recompressed by a highly-efficient compressor made up of a few chirped multilayer mirrors [24].

Furthermore, the threshold intensity for optical damage of transparent materials increases even faster than the $1 / \tau^{1 / 2}$ scaling law for laser pulse durations $\tau$ decreasing below 20 ps [25]. For pulses longer than a few tens of picoseconds, the generally accepted picture of bulk damage to defect-free dielectrics involves the heating of conduction-band electrons by the incident radiation and transfer of this energy to the lattice. Damage occurs when the deposited heat is sufficient to melt, boil, or fracture the dielectric material. Because the controlling rate is that of thermal conduction through the lattice, this model predicts [77] a $\tau^{1 / 2}$ dependence of the threshold damage fluence upon pulse duration $\tau$. For large-band-gap materials, such as dielectric materials, a change in the damage mechanism and morphology for pulses shorter than 20 ps can be observed. For pulses shorter than $\sim 20 \mathrm{ps,} \mathrm{electrons} \mathrm{have} \mathrm{insufficient} \mathrm{time} \mathrm{to} \mathrm{couple}$ to the lattice during the laser pulse and a continuously decreasing threshold occurs, associated with a gradual transition from the long pulse, thermally dominated regime, to an ablative regime dominated by collisional and multiphoton ionization, and plasma formation [37]. 
As a consequence, exposing the nonlinear crystal to higher intensities allows the same OPA gain to be attained with a shorter crystal and hence over a broader bandwidth [26]. A simple gain calculation of an OPA can be derived from the analytical solution of the coupled-wave equations, assuming the slowly-varying-envelope approximation, flat top spatial and temporal profiles and neglecting pump depletion. The intensity gain G of an OPA can be expressed as

$$
\mathrm{G}=1+(\mathrm{gL})^{2}\left(\frac{\sinh (\alpha)}{\alpha}\right)^{2}
$$

with

$$
g=4 \pi d_{\text {eff }} \sqrt{\frac{I_{p}}{2 \varepsilon_{0} n_{p} n_{s} n_{i} c \lambda_{s} \lambda_{i}}}
$$

and

$$
\alpha=\sqrt{(\mathrm{gL})^{2}-\left(\frac{\Delta \mathrm{kL}}{2}\right)^{2}}
$$

where

$$
\Delta \mathrm{kL} \equiv\left(\mathrm{k}_{\mathrm{p}}-\mathrm{k}_{\mathrm{s}}-\mathrm{k}_{\mathrm{i}}\right) \mathrm{L}
$$

is the scalar phase mismatch of the wave vectors of the pump laser $\mathrm{k}_{\mathrm{p}}$, the signal $\mathrm{k}_{\mathrm{s}}$ and the idler wave $k_{i}$. $L$ is the interaction length in a nonlinear crystal, $d_{\text {eff }}$ is the effective second order nonlinear coefficient of the nonlinear crystal, $I_{p}$ is the pump intensity, $\varepsilon_{0}$ is the permittivity of free space, $n_{p}$ is the refractive index of the pump laser, $n_{s}$ is the refractive index of the signal, $\mathrm{n}_{\mathrm{i}}$ is the refractive index of the idler, $\mathrm{c}$ is the speed of light in vacuum, $\lambda_{\mathrm{p}}$ is the pump laser wavelength, $\lambda_{\mathrm{s}}$ the signal wavelength and $\lambda_{\mathrm{i}}$ the idler wavelength. $\mathrm{G}$ can be simplified to $\mathrm{G}^{\prime}$ assuming exact phase matching $(\Delta \mathrm{k}=0)$ as

$$
\mathrm{G}^{\prime} \approx(\mathrm{gL})^{2}\left(\frac{\sinh (\mathrm{gL})}{\mathrm{gL}}\right)^{2}=\sinh ^{2}(\mathrm{gL})
$$

and expresses directly the relation between the pump intensity and the length of the OPA crystal. As can be seen in equation 1-6, G' returns the same value if gL is constant.

$\mathrm{gL}$ can be related to the pump intensity $\mathrm{I}_{\mathrm{p}}$ and crystal length $\mathrm{L}$ as

$$
\mathrm{gL} \propto \sqrt{\mathrm{I}_{\mathrm{p}} \mathrm{L}}
$$

Therefore the OPA gain is constant for

$$
\sqrt{\mathrm{I}_{\mathrm{p}} \mathrm{L}}=\text { const. }
$$

and shows immediately that a ten times higher pump intensity leads to a 3.16 (= square root of 10) times shorter OPA crystal in order to achieve the same gain while supporting at the same time a larger bandwidth due to better phase matching conditions in the shorter OPA crystal. 
Figure 1-4 shows the calculated wavelength dependent gain in a bulk lithium niobate crystal for a $20 \mathrm{~mJ}$ pump pulse and different combinations of pump pulse durations, pump beam diameters and crystal lengths required to achieve the same gain in a bulk lithium niobate crystal. It can be seen that the supported bandwidth increases drastically for shorter crystals.

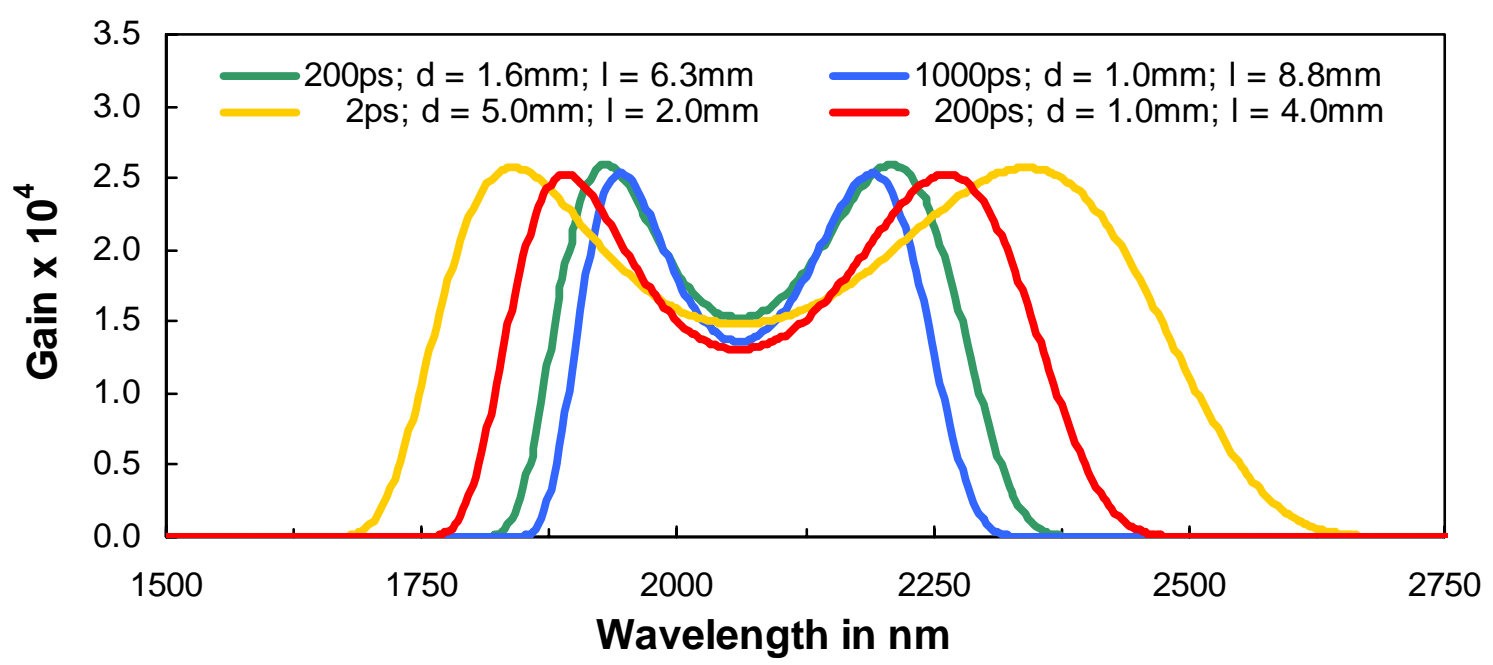

Fig. 1-4 Calculated gain over the wavelength for a $20 \mathrm{~mJ}$ pump pulse and different combinations of pump pulse durations, pump beam diameters and crystal lengths required to achieve the same gain in a bulk lithium niobate crystal. It can be seen that the supported bandwidth increases drastically for shorter crystals (d: beam diameter; l: crystal length). 


\section{Yb:YAG as laser active medium}

As a quasi-three-level system $\mathrm{Yb}$ :YAG requires high pump power densities $>1.5 \mathrm{~kW} / \mathrm{cm}^{2}$ but since high power pump diodes for the absorption bandwidth around $940 \mathrm{~nm}$ are available, $\mathrm{Yb}$ :YAG has become a popular laser medium for $\mathrm{cw}$ high power and even pulsed high energy lasers especially based on the thin disk technology. Currently Yb:YAG disk based laser demonstrated cw output powers of more than $8 \mathrm{~kW}$ [28], showed $280 \mathrm{~mJ}$ pulse energy in a cavity dumped system with $25 \mathrm{~ns}$ pulse durations at a repetition rate of $100 \mathrm{~Hz}$ [78] and are commercially available with pump powers of up to $12 \mathrm{~kW}$ at Dausinger \& Giesen GmbH. Yb:YAG exhibits a complete set of properties favorable for high-power diode-pumping [79]:

- very low quantum defect (91\% quantum efficiency)

- very low fractional heating

- broad absorption bands (10 nm@940 nm)

- high doping levels possible without quenching (>20\%)

- no excited-state absorption or upconversion

- $940 \mathrm{~nm}$ pump enables use of very reliable InGaAs diodes

- high thermal conductivity of YAG $\left(6.6 \mathrm{Wm}^{-1} \mathrm{~K}^{-1} @ 3 \% \mathrm{Yb}\right.$ doping $)$

- high tensile strength of the host material

- long lifetime of upper laser level $(\sim 1 \mathrm{~ms})$

- broad emission bands ( $\sim 10 \mathrm{~nm} ;<1$ ps pulses possible)

Other rather new laser materials as $\mathrm{Yb}$-doped sesquioxides ( $\left.\mathrm{Yb}: \mathrm{Sc}_{2} \mathrm{O}_{3} ; \mathrm{Yb}: \mathrm{Lu}_{2} \mathrm{O}_{3} ; \mathrm{Yb}: \mathrm{Y}_{2} \mathrm{O}_{3}\right)$ could also be considered as laser media in high gain thin disk amplifiers impressing through high quantum efficiency ( 95\%), high heat conduction $\left(\sim 11 \mathrm{Wm}^{-1} \mathrm{~K}^{-1} @ 3 \% \mathrm{Yb}\right.$ doping), large bandwidth (11.6 - $14.4 \mathrm{~nm})$ and higher emission cross section than Yb:YAG [80].

\subsection{Disk laser}

All these advantages are perfectly combined in the 1994 presented thin disk based laser and amplifier head [81] developed at the Institut für Strahlwerkzeuge (IFSW) at the Technical University Stuttgart and nowadays commercially available at the company Dausinger + Giesen $\mathrm{GmbH}$.

The general principle of the disk laser head is shown in figure 2-1. The pump radiation produced by laser diodes is first homogenized either by fiber coupling or by focusing into a quartz rod and imaged on the disk using a collimation optic and the parabolic mirror inside the laser head. The disk is coated on the backside for reflecting the pump and signal wavelength. Therefore the unabsorbed part of the pump radiation is recollimated at the opposite side of the parabolic mirror and redirected via two mirrors to another part of the parabolic mirror refocusing it again on the disk. This reimaging is repeated until the complete pump light is absorbed in the disk. This technically mature scheme can be used for small disks 100 to $200 \mu \mathrm{m}$ thin with a few millimeters in diameter cooled via a substrate or larger few millimeter thick free standing disks with $60 \mathrm{~mm}$ in diameter directly water cooled from the back- 
side. Figure 2-2 shows the commercial $5 \mathrm{~kW}$ laser head from Trumpf Laser GmbH \& Co. KG together with the pump radiation absorption scheme.

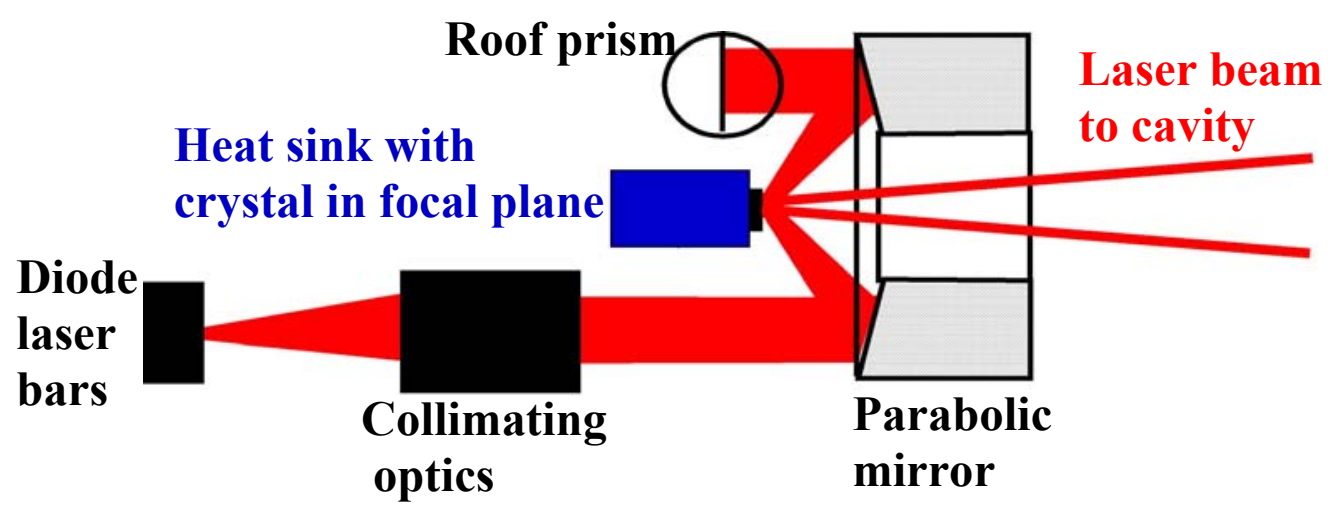

Fig. 2-1 Schematical setup of the disk laser head and side view of the pump light reimaging technique.

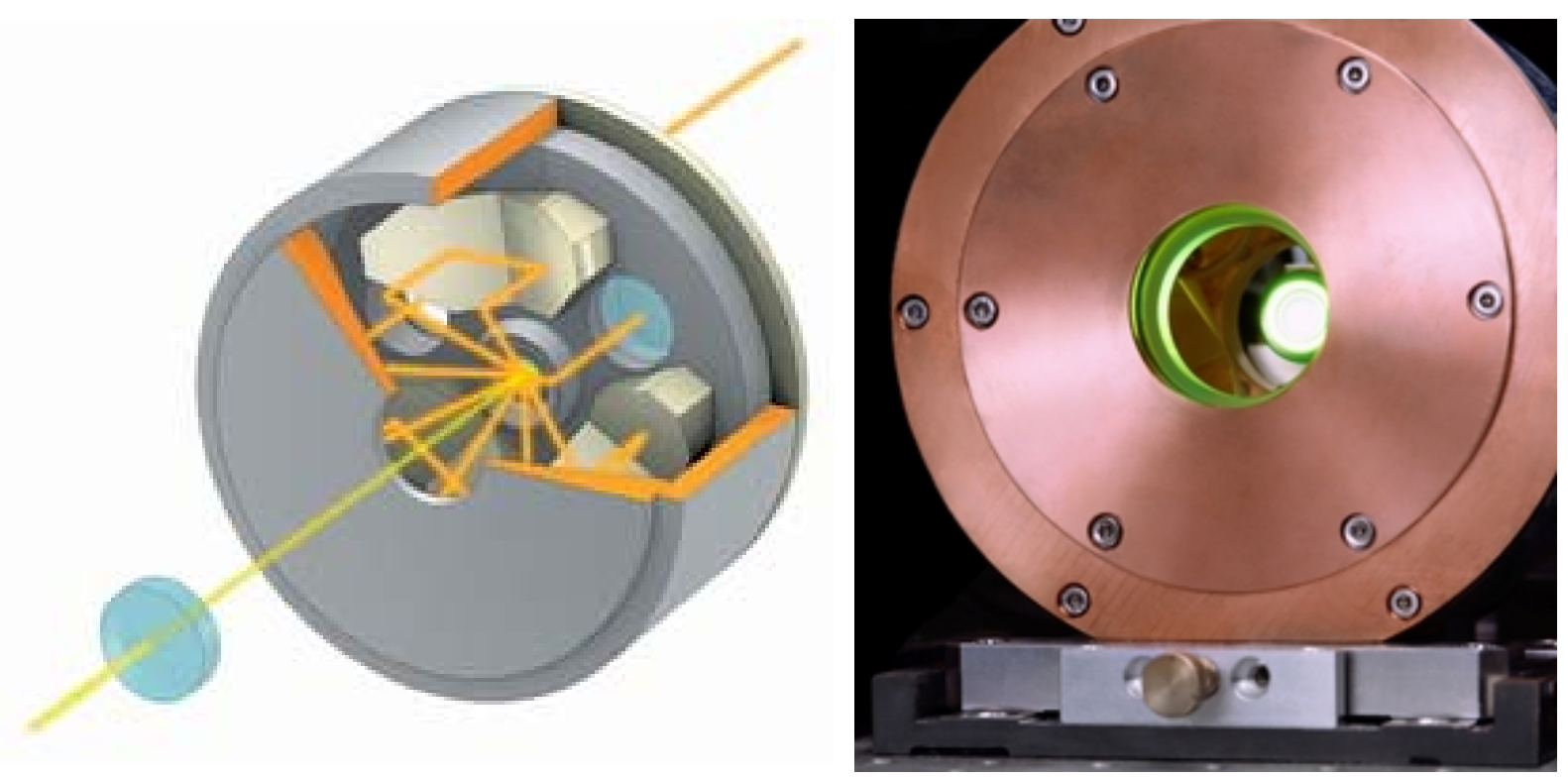

Fig. 2-2 Principle of the disk laser and the pump light reimaging technique (left side) and the $5 \mathrm{~kW}$ laser head by courtesy of Trumpf Laser GmbH + Co. KG.

Scaling a laser towards high repetition rates while still aiming for high energy picosecond pulses means not only dealing with intensity-caused difficulties just like optical damage or nonlinear effects such as self phase modulation or self focusing but also with exceedingly high thermal loads in the laser gain medium. So far no other laser concept has shown such a good scalability towards high average powers with energies well above the millijoule range than the thin disk laser technology. Through the efficient one dimensional heat removal a disk laser accepts extremely high pump densities of $1 \mathrm{MW} / \mathrm{cm}^{3}$ while still keeping thermal induced distortions and lensing of the laser media low allowing outstanding beam qualities even in multi pass arrangements.

Energy scaling and power scaling can be easily realized via larger disk diameters and enable scaling in two dimensions not only in one as it is mostly the case for slab laser arrangements (scaling via the length of the slab). Figure 2-3 shows the laser head of Dausinger + Giesen $\mathrm{GmbH}$ for cw output powers of up to $12 \mathrm{~kW}$ with possible disk diameters as large as $60 \mathrm{~mm}$. 
Simulations performed at the IFSW Stuttgart show that output powers in the range of tens of kilowatts average power will be possible using a single disk [82].
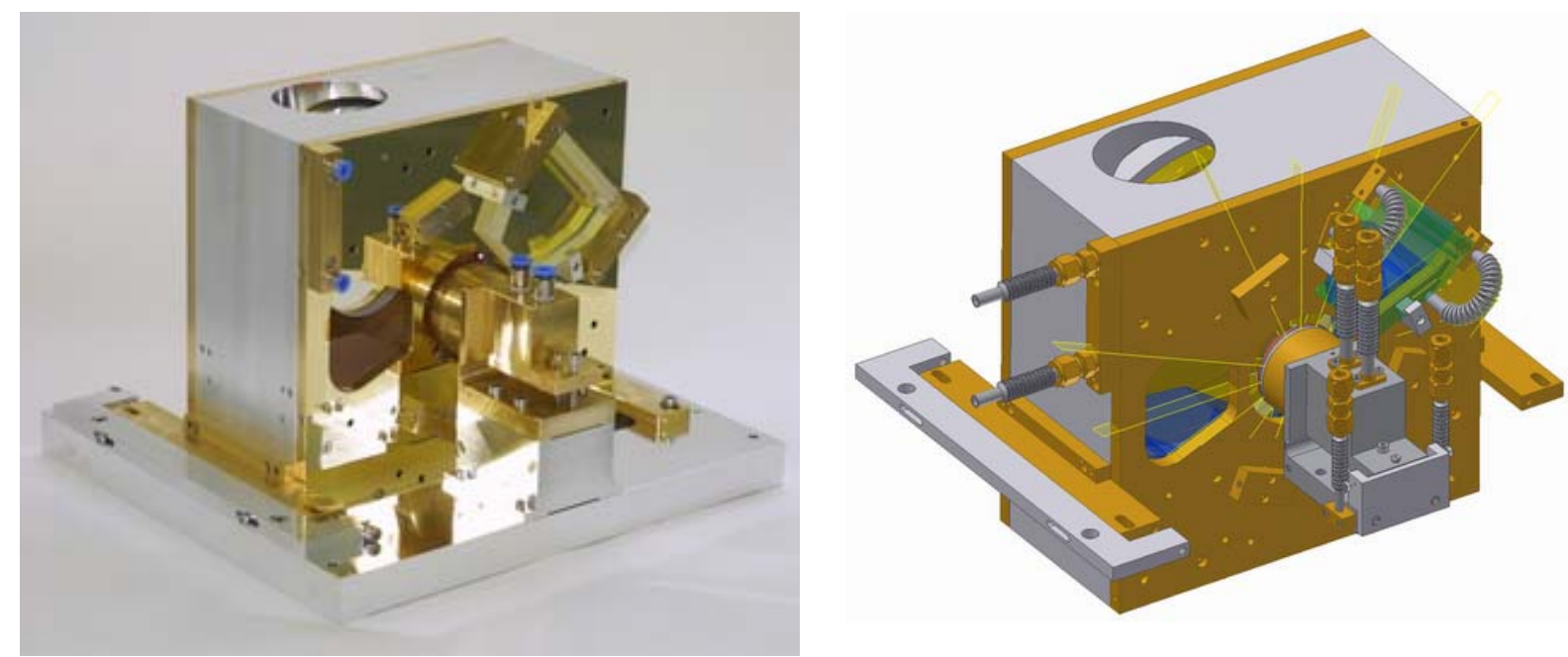

Fig. 2-3 The commercial $12 \mathrm{~kW}$ cw disk laser head by courtesy of the Dausinger + Giesen GmbH.

Another exciting aspect of a disk based laser is the small longitudinal extension of the gain medium allowing the amplification of short pulses without facing problems due to nonlinearities and large B-integral accumulation. Therefore high peak power sources are possible without cost intensive large gratings in stretcher and compressor setups. The disadvantage of low amplification in the thin disk is easily compensated by the large possible pump densities as it is shown in chapter 8 . 


\section{Seed generation}

The regenerative amplifier system presented in this work was especially designed to pump an OPA and therefore needs to be temporal synchronized with the designated seed source of the OPA. For a precise synchronization the all-optical approach introduced by [22] has been chosen where the seed source of the regenerative amplifier and the OPA derive from the same oscillator. Electronic synchronization of the pump laser and OPA seed source was used in $[20,83,84]$, where a broadband Ti:sapphire oscillator, employed as OPCPA seed, was synchronized with the master oscillator of a picosecond pump laser based on Nd:YAG using a complex electronic servo loop [85]. State-of-the art active synchronization loops [83], based on pricy $\mathrm{GHz}$ electronics, make it possible to keep the root-mean-square timing jitter between the seed and pump pulses on a sub-picosecond level. Whereas this locking precision satisfies well the demands of OPCPA pumped by Nd-based picosecond lasers (pulse duration range 20-100 ps), it is insufficient for use with subpicosecond Yb-based pump lasers.

The first optical synchronization has been reported in [86] where a fraction of a broadband seed pulse centered at $760 \mathrm{~nm}$ from a 70-MHz Ti:sapphire oscillator was frequency-shifted in a photonic crystal fiber to enable synchronized seeding of a picosecond Nd:YAG pump laser allowing to abstain from all electronic synchronization techniques. Later, the Ti:sapphire oscillator used in [86] was developed further by changing the chirped mirrors to provide a broader spectrum containing enough signal in the infrared to seed a regenerative amplifier directly without the intermediate step of using a frequency shifter. In table 3-1 the properties of electronic and optical synchronization methods are comparative summarized.

\begin{tabular}{|c|c|c|}
\hline & $\begin{array}{c}\text { electronic } \\
\text { synchronization }\end{array}$ & $\begin{array}{c}\text { optical } \\
\text { synchronization }\end{array}$ \\
\hline $\begin{array}{l}\text { Timing jitter } \\
\text { (short term) }\end{array}$ & $<$ few ps & $<1$ ps \\
\hline $\begin{array}{l}\text { Timing drift } \\
\text { (long term) }\end{array}$ & several tens of ps & several ps \\
\hline $\begin{array}{l}\text { Wavelength } \\
\text { tunability }\end{array}$ & flexible & $\begin{array}{c}\text { flexible within } \\
\text { oscillator spectrum }\end{array}$ \\
\hline Coarse timing & $\begin{array}{l}\text { daily realignment } \\
\text { required }\end{array}$ & fixed all time \\
\hline $\begin{array}{l}\text { Seed energy to } \\
\text { pump energy }\end{array}$ & high & low (ASE problem) \\
\hline Handling & complicated & simple \\
\hline Cost & $\begin{array}{l}\text { two oscillator and } \\
\text { locking electronics }\end{array}$ & $\begin{array}{l}\text { ultra broadband } \\
\text { oscillator }\end{array}$ \\
\hline
\end{tabular}

Tab. 3-1 Comparison of electronic and optical synchronization methods. 
For seeding the designated OPA and the regenerative amplifier presented in this work a home made ultra-broadband 6 fs-Ti:sapphire oscillator [87] described in chapter 3.2 has been used. To be independent of the Ti:sapphire oscillator development for the first experiments a commercial Yb:KGW laser with $70 \mathrm{MHz}$ repetition rate described in chapter 3.1 has been used.

\subsection{Yb:KGW oscillator}

To be independent of the ultra broadband Ti:sapphire seed oscillator being developed and described in chapter 3.2 for the first experiments a commercial ytterbium doped potassium gadolinium tungstate $(\mathrm{Yb}: \mathrm{KGW})$ oscillator from Positive Light was used to seed the regenerative amplifier. At $70 \mathrm{MHz}$ repetition rate, pulse energies of $\sim 5 \mathrm{~nJ}$ with 450 ps pulse duration were measured. The spectral bandwidth was measured to be $\sim 3.5 \mathrm{~nm}$ at a center wavelength of $1028 \mathrm{~nm}$ and corresponds to a time bandwidth limited pulse duration of $445 \mathrm{fs}$.

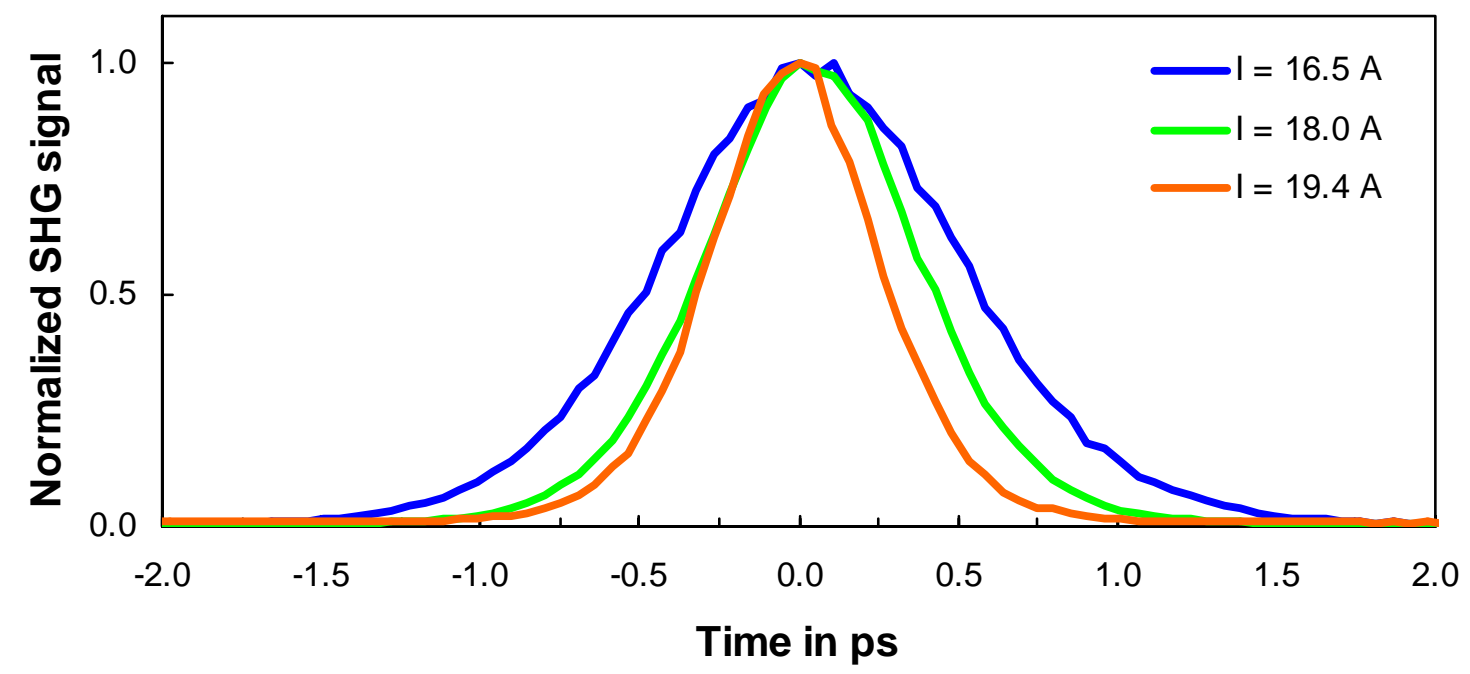

Fig. 3-1 Measured second harmonic (SHG) autocorrelation traces of the Yb:KGW oscillator in dependence of the diode pump current.

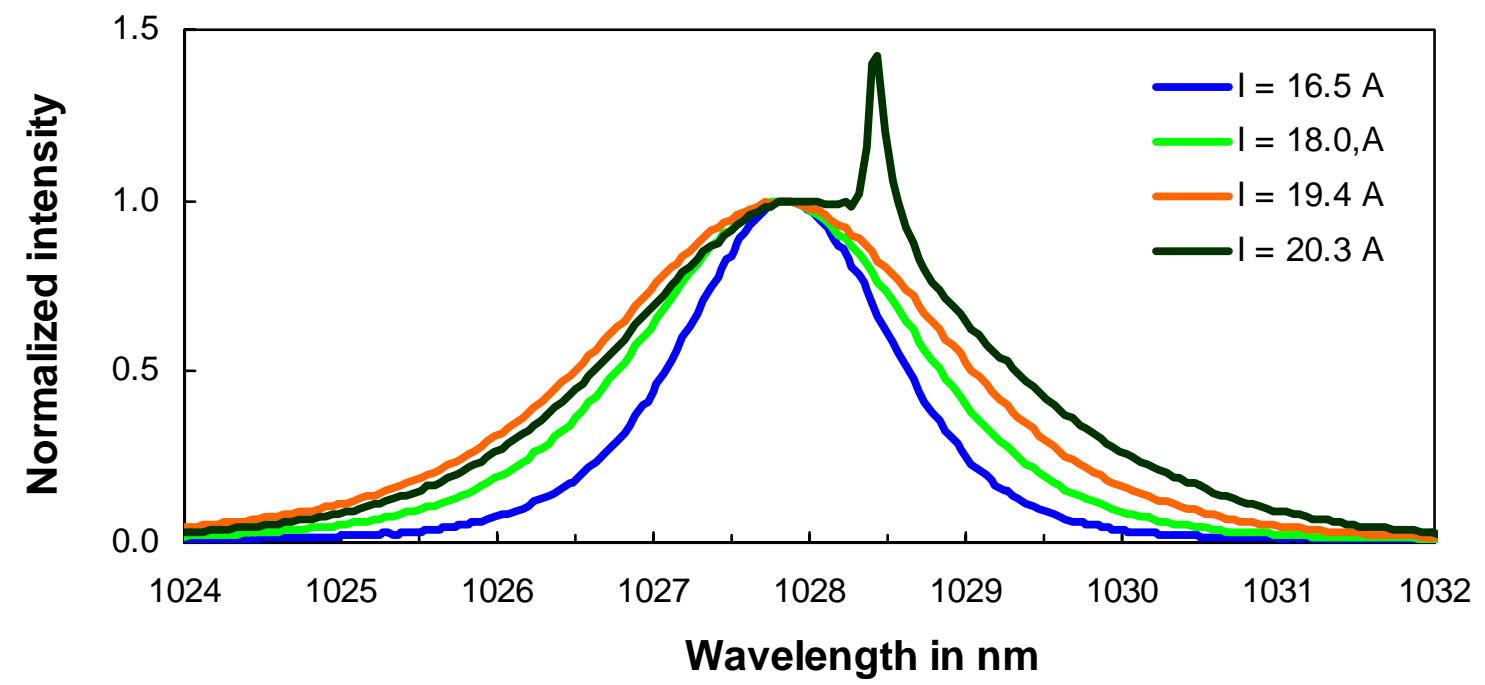

Fig. 3-2 Measured output spectrum of the Yb:KGW oscillator in dependence of the pump current. Above a pump current of $20 \mathrm{~A}$ a cw spike was detected. 
The passively mode-locked laser [88] was directly diode pumped and figure 3-1 and figure 32 show the pump current dependent pulse duration and the correspondent output spectra, respectively. Above a pump current of $20 \mathrm{~A}$, a cw spike was detected through an additional upcoming cw background of the at this point not completely mode-locked oscillator. To run the laser without $\mathrm{cw}$ background and without driving the pump diodes at a too high current the laser was operated at $18.0 \mathrm{~A}$, resulting in a pulse duration of $530 \mathrm{fs}$.

\subsection{Ti:sapphire oscillator}

The seed spectrum for both the OPA and the regenerative amplifier are generated directly from a mirror dispersion-controlled Kerr-lens mode-locked Ti:sapphire oscillator described in [87] with an ultra-broad and smooth spectra. The total output power reaches $250 \mathrm{~mW}$ at a repetition rate of $70 \mathrm{MHz}$ with pulse durations of $\sim 6 \mathrm{fs}$. The beam path of the prism-less Ti:sapphire laser and extracavity dispersion-compensation setup is shown in figure 3-3 and the layout is displayed in figure 3-4. The measured complete spectra of the Ti:sapphire seed oscillator is displayed in figure 3-5.

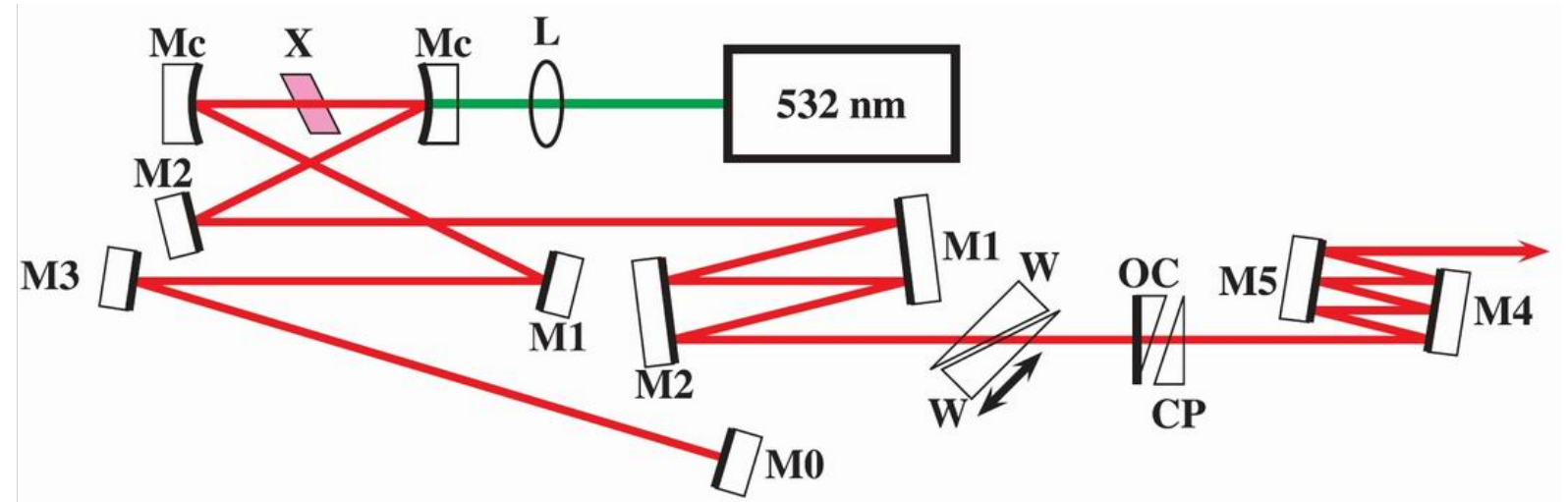

Fig. 3-3 The beam path of the prism-less Ti:sapphire laser and extra cavity dispersion-compensation setup [87]. CP, compensating plate; L, incoupling lens; M 0-5, chirped mirrors; Mc, 50 mm folding mirror; OC, output coupler; $X$, Ti:sapphire crystal; W, wedged plate.

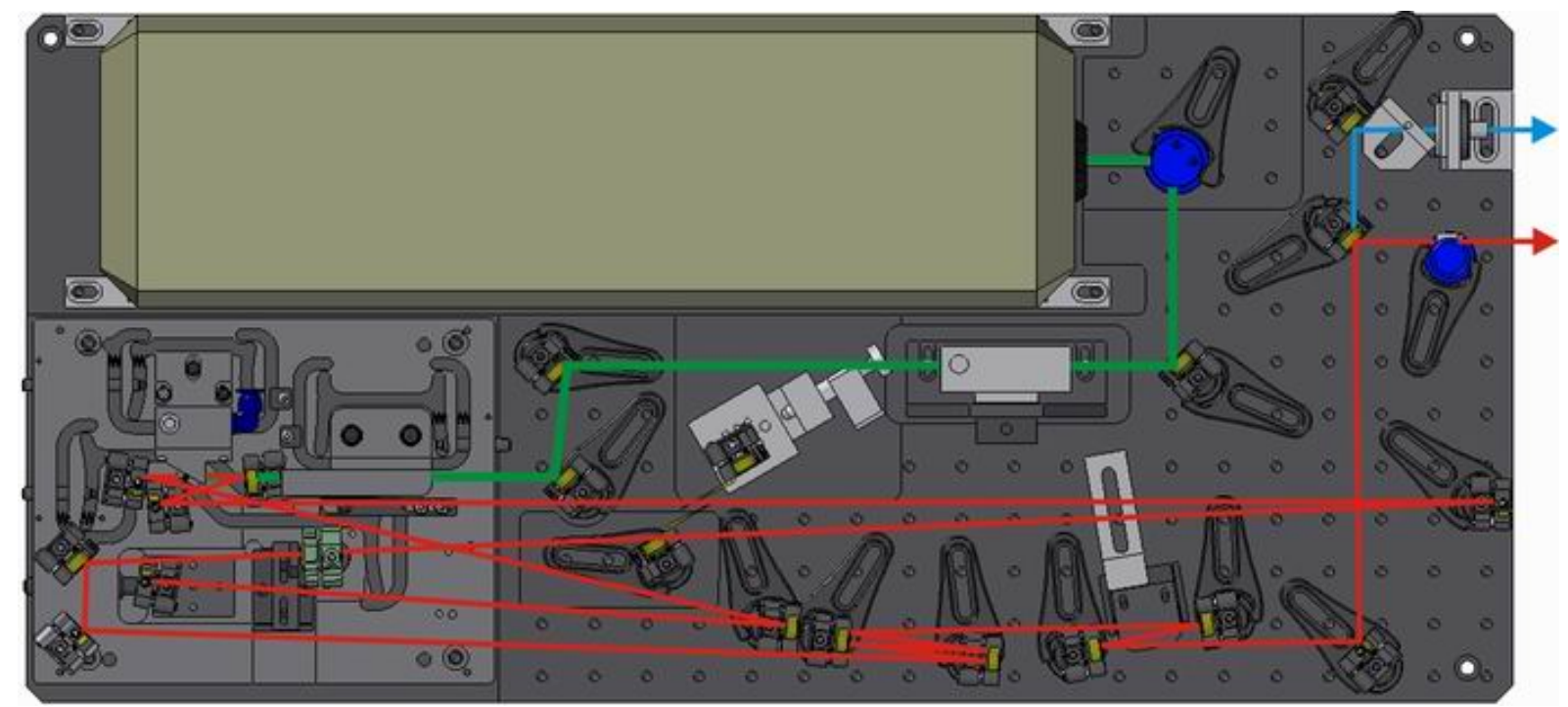

Fig. 3-4 Schematical layout of the Ti:sapphire seed oscillator. 


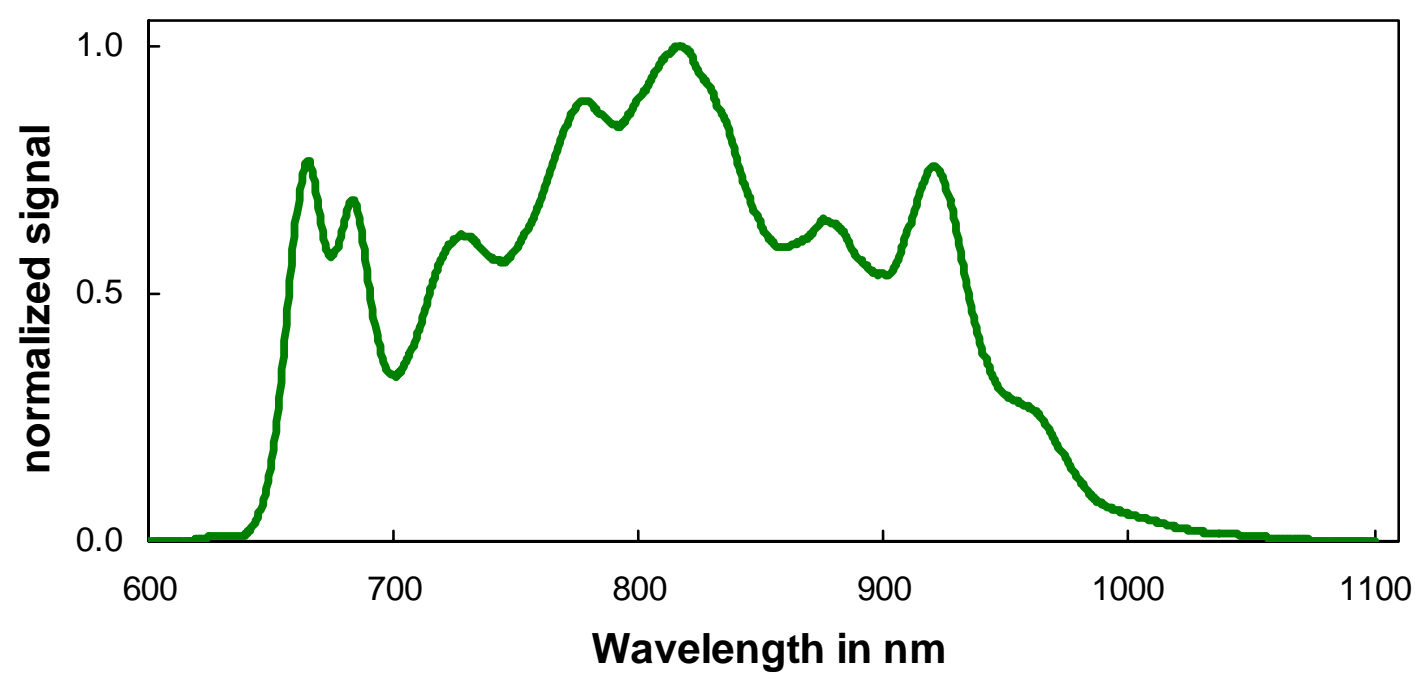

Fig. 3-5 Measured spectra of the Ti:sapphire seedlaser.

The oscillator output is simultaneously broadened by SPM to more than an octave and the difference frequency (DFG) between the high and low frequency components is generated in a $2 \mathrm{~mm}$ long magnesium-oxide-doped periodically poled lithium niobate crystal (PP$\mathrm{MgO}: \mathrm{LN}$ ) to measure and stabilize the carrier envelope offset phase of the oscillator pulses [89]. Two dichroic mirrors are used to separate the spectral components. The IR component contains the carrier-envelope phase (CEP) information while the near IR component is used for seeding the disk based regenerative amplifier. Figure 3-6 shows how the different spectral components are splitted off. The measured infrared components of the Ti:Saphire oscillator interesting for seeding the regenerative amplifier around $1000 \mathrm{~nm}$ are shown in figure 3-7. The inset of figure 3-7 shows the measured beam profile of the infrared components.

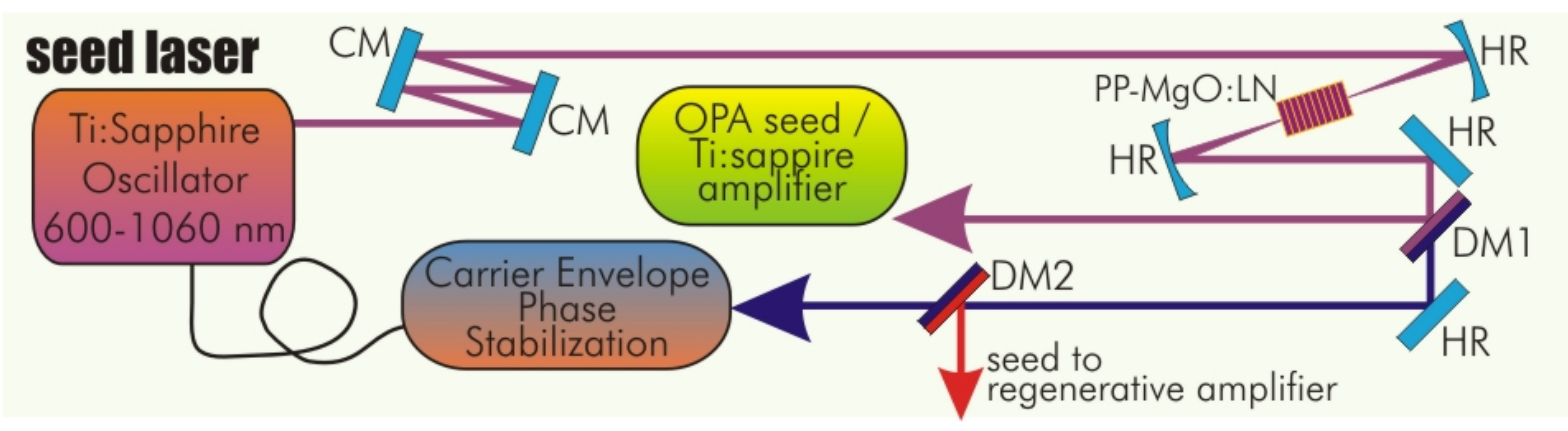

Fig. 3-6 Seed laser set-up (CM: chirped mirror, DM: dichroic mirror).

The infrared seed spectrum is simultaneously used in the laboratory for an amplifier based on $\mathrm{Nd}$ :YLF $\left(\lambda_{\mathrm{s}}=1053 \mathrm{~nm}\right.$ ) for pumping an already existing OPA [19]. Being able to use the full spectral components provided for each amplifier system by the Ti:sapphire oscillator the infrared seed spectra is split by an edge filter. The spectral components above $1043 \mathrm{~nm}$ for the $\mathrm{Nd}$ :YLF laser are transmitted and below $1043 \mathrm{~nm}$ for the Yb:YAG disk based amplifier reflected. Figure 3-8 shows the spectral components separated by the edge filter in comparison to the entire infrared seed spectrum provided by the Ti:sapphire oscillator. 


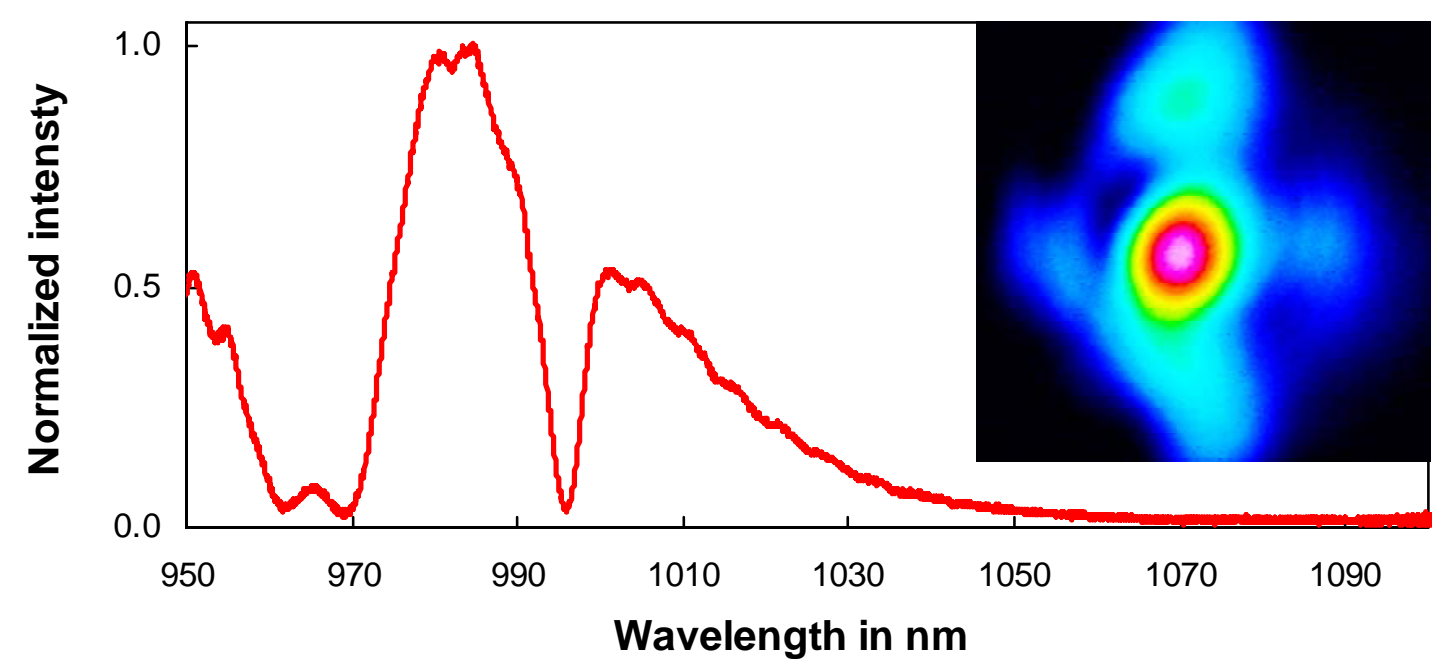

Fig. 3-7 Measured infrared components of the Ti:Sapphire oscillator interesting for seeding the regenerative amplifier around $1000 \mathrm{~nm}$.

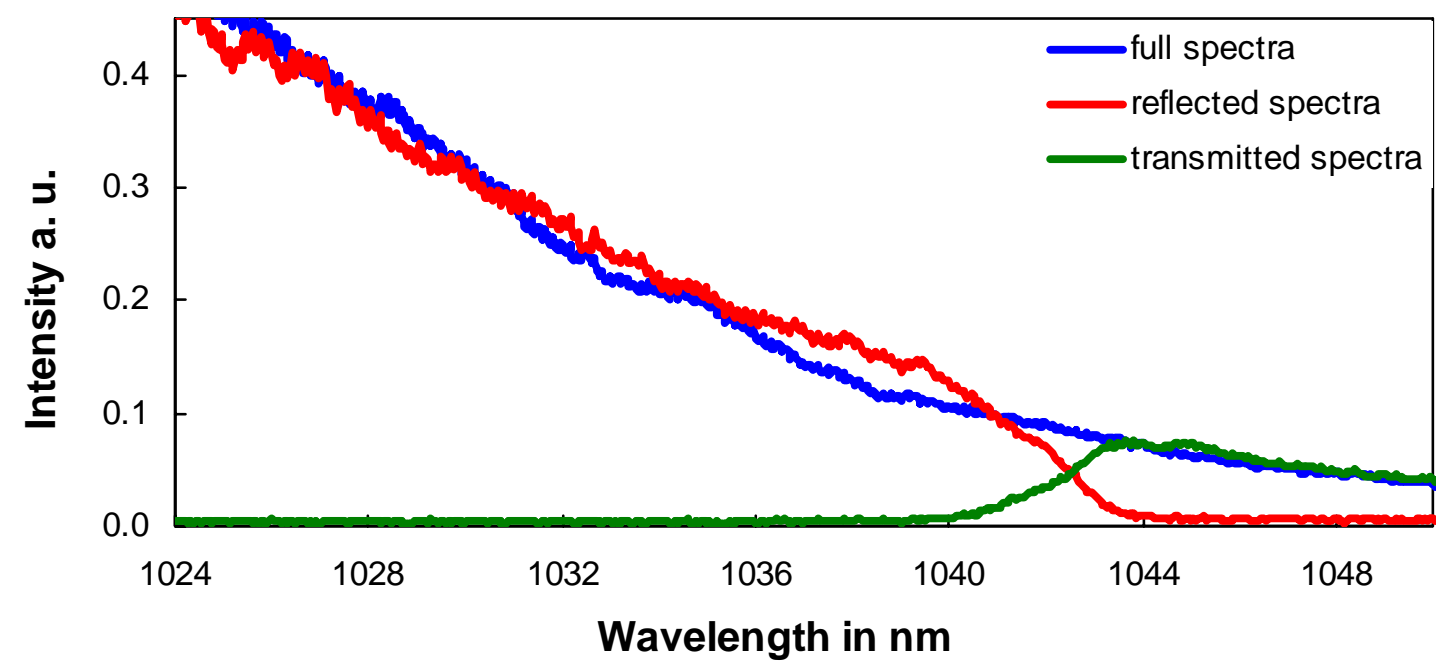

Fig. 3-8 Spectral components separated by the edge filter in comparison to the entire infrared seed spectrum provided by the Ti:sapphire oscillator.

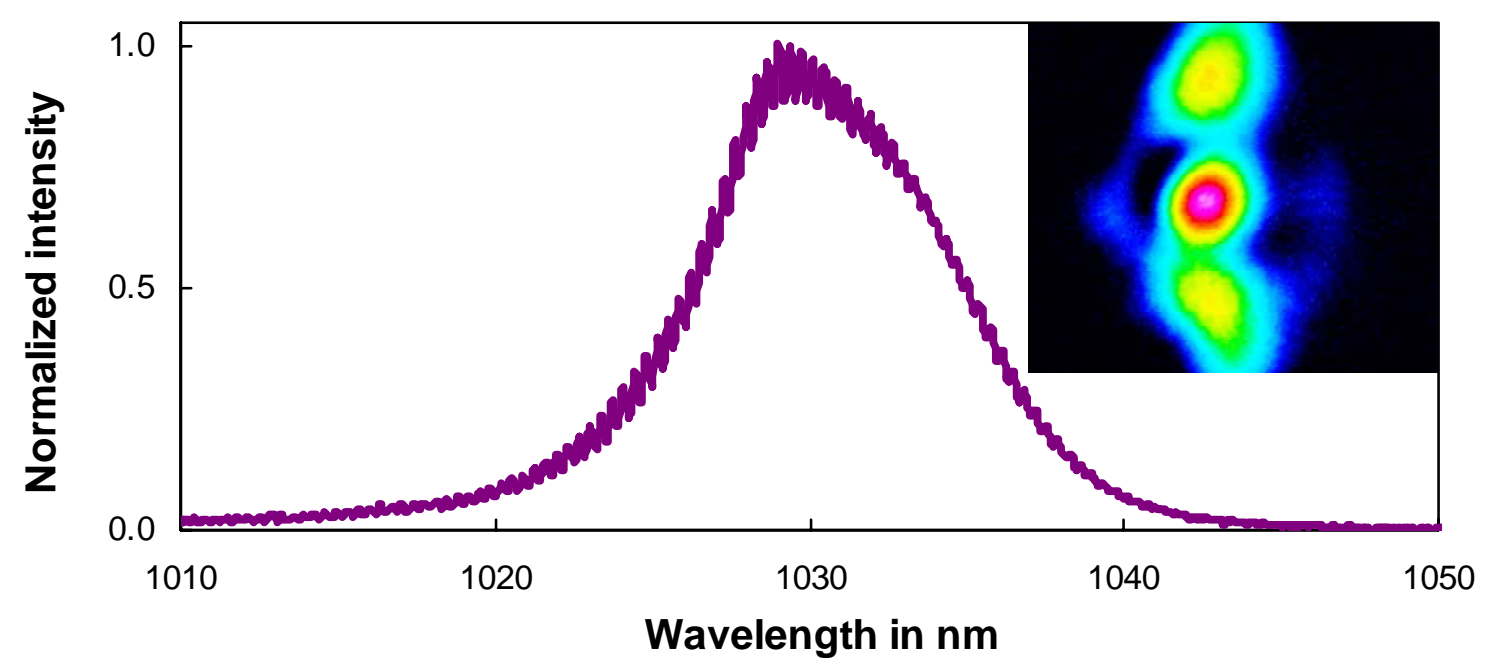

Fig. 3-9 Spectra and beam profile around the wavelength of $1030 \mathrm{~nm}$ sliced out with an $10 \mathrm{~nm}$ broad interference filter. 
An interference filter for $10 \mathrm{~nm}$ bandwidth with $~ 50 \%$ transmittance was used to determine the entire seed energy and beam profile shown in figure 3-9 within the spectral acceptance of the Yb:YAG amplifier. The resultant seed energy in the spectral region of the $\sim 5.3 \mathrm{~nm}$ broad gain bandwidth of $\mathrm{Yb}: Y A G$, centered at $1030 \mathrm{~nm}$, was measured to be $\sim 2 \mathrm{pJ}$.

\subsection{Gain narrowing}

The emission band of $\mathrm{Yb}: \mathrm{YAG}$ around $1030 \mathrm{~nm}$ allows an amplification of a bandwidth of $\sim 5.3 \mathrm{~nm}$ which correspondents to a time bandwidth limited (TBL) Gaussian pulse duration of 294 fs. The pulse energy delivered by the Ti:Sapphire oscillator shown in the spectral region between $1027.5 \mathrm{~nm}$ and $1032.5 \mathrm{~nm}$ (figure 3-9) is estimated to be around $1 \mathrm{pJ}$. When amplifying pulses with a broad bandwidth close to the supported bandwidth of the laser gain medium one has to take into account that different spectral components of the pulse experience different gain leading to an elongation of the duration for an unchirped pulse. In a linear system without saturation the center region of the optical spectrum experiences a higher gain than the spectral wings resulting in a narrower bandwidth. The limited bandwidth of Yb:YAG is clearly visible in the fluorescence spectrum of the disk amplifier in figure 3-10.

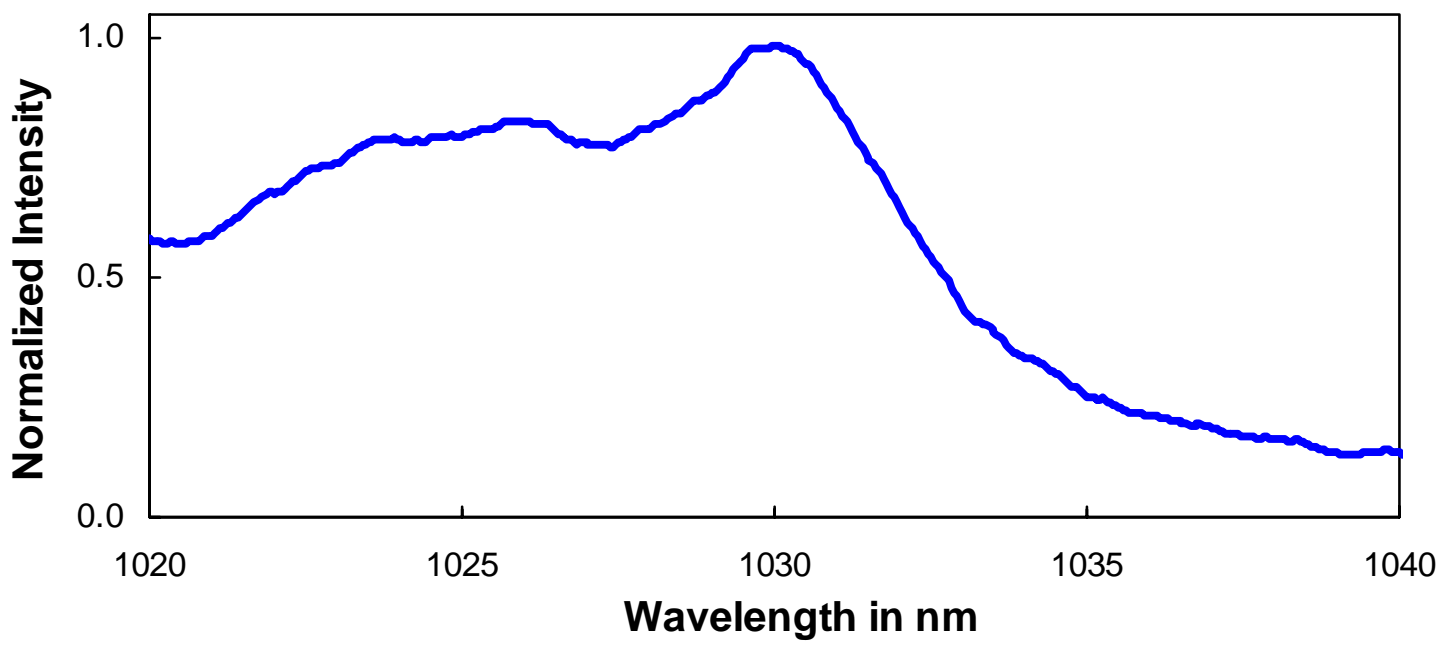

Fig. 3-10 Fluorescence spectrum of the Yb:YAG disk amplifier.

Assuming an unchirped Gaussian pulse $\tau_{\text {in }}$ at the input of the amplifier with a Gaussian small signal gain distribution the output pulse duration $\tau_{\text {out }}$ is given by

$$
\tau_{\text {out }}=\sqrt{\tau_{\text {in }}^{2}+\frac{(16 \ln 2) \ln \mathrm{a}_{0}}{\Delta \omega_{\mathrm{g}}}}
$$

where $\mathrm{a}_{0}$ is the overall gain and $\Delta \omega_{\mathrm{g}}$ is the spectral FWHM of the gain curve determined by the gain medium [97]. With the given supported bandwidth $\lambda_{\mathrm{g}}$ of the gain medium at the center wavelength $\lambda_{\mathrm{c}}$ the frequency width $\Delta \omega_{\mathrm{g}}$ is

$$
\Delta \omega_{\mathrm{g}}=\frac{2 \pi \mathrm{c} \cdot \Delta \lambda_{\mathrm{g}}}{\lambda_{\mathrm{c}}^{2}}
$$


The minimal reachable pulse duration after amplifying the seed pulse with a bandwidth of $\sim 5.3 \mathrm{~nm}$ (maximum accepted bandwidth of Yb:YAG) and a pulse energy of $1 \mathrm{pJ}$ (estimated seed energy of the Ti:Sapphire oscillator) to an energy level of approximately $5 \mathrm{~mJ}$ $\left(\mathrm{a}_{0}=5 \cdot 10^{9}\right)$ given by gain narrowing was calculated to be $1.69 \mathrm{ps}$ which correspondents to a final TBL bandwidth of $0.92 \mathrm{~nm}$. The calculated pulse duration of $\sim 1.7 \mathrm{ps}$ is still well below the desired goal of this work to reach pulse durations below 2 ps and no further measures for broadening the amplified spectra have to be taken into account. Broadening of the spectra could be achieved via intracavity filters suppressing the center wavelength with the highest gain or spectral shaping of the input pulse. Both methods aim for higher amplification of the spectral wings compared to the center wavelength. Figure 3-11 shows the final pulse duration calculated according to equation 3-1 caused by gain narrowing for TBL input pulses of different durations amplified by a factor of $5 \cdot 10^{9}$.

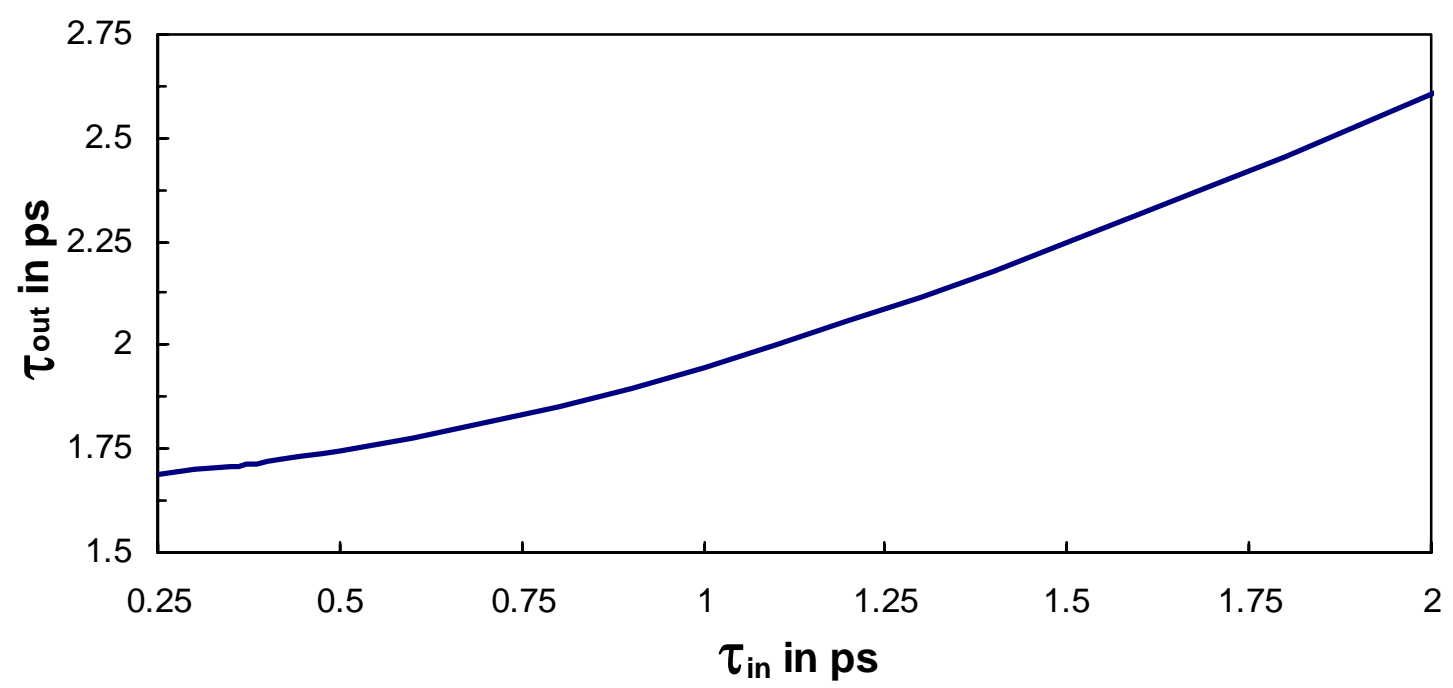

Fig. 3-11 Final pulse duration caused by gain narrowing for input pulses of different duration amplified by a factor of $5 \cdot 10^{9}$ at a center wavelength of $1030 \mathrm{~nm}$. 


\section{Preliminary considerations about the regenerative amplifier}

Considering amplification without chirped pulse amplification (CPA) [27] nonlinear effects caused by the bulk material inside the regenerative amplifier cavity can lead to undesired temporal pulse distortion via self phase modulation (SPM) and optical damage entailed by self focusing (SF) [90]. Both SPM and SF have their physical origin in a nonlinear polarization generated in the medium resulting in the temporal intensity $\mathrm{I}(\mathrm{t})$ dependent refractive index $n$

$$
\mathrm{n}=\mathrm{n}_{0}+\overline{\mathrm{n}}_{2} \mathrm{I}(\mathrm{t})
$$

where $\overline{\mathrm{n}}_{2}$ the nonlinear refractive index can be described with the permittivity of free space $\varepsilon_{0}$ and the speed of light $\mathrm{c}$ as

$$
\overline{\mathrm{n}}_{2}=\frac{2 \mathrm{n}_{2}}{\varepsilon_{0} \mathrm{cn}}
$$

The quantity $\mathrm{n}_{2}$ is called nonlinear index coefficient and describes the strength of the coupling between the electric field and the refractive index. The most often used quantities are $\mathrm{n}_{2}$ in esu units and $\overline{\mathrm{n}}_{2}$ in $\mathrm{cm}^{2} / \mathrm{W}$. The conversion factor between the two quantities is:

$$
\overline{\mathrm{n}}_{2}\left(\mathrm{~cm}^{2} / \mathrm{W}\right)=\frac{2}{300^{2} \mathrm{n}_{0}} \sqrt{\frac{\mu_{0}}{\varepsilon_{0}}} \mathrm{n}_{2}(\mathrm{esu}) \approx \frac{8.378}{\mathrm{n}_{0}} \cdot 10^{-3} \mathrm{n}_{2}(\mathrm{esu})
$$

with the permeability of free space $\mu_{0}$. The nonlinear refractive index is a result of an optical nonlinearity of third order and can be described with the material dependent third-order susceptibility $\chi^{(3)}$ :

$$
\mathrm{n}_{2}=\frac{3 \chi^{(3)}}{8 \mathrm{n}_{0}}
$$

Table 4-1 gives some examples of $\overline{\mathrm{n}}_{2}$ for different materials.

\begin{tabular}{ccccc}
\hline Material & $\mathrm{n}_{0}$ & $\overline{\mathrm{n}}_{2}\left(\mathrm{x}^{\left.-10^{-16} \mathrm{~cm}^{2} / \mathrm{W}\right)}\right.$ & $\lambda(\mathrm{nm})$ & Reference \\
\hline YAG & 1.82 & 6.21 & 1064 & {$[91]$} \\
$\mathrm{YAlO}_{3}$ & 1.93 & 7.30 & 1064 & {$[91]$} \\
$\mathrm{BBO}$ & 1.6 & 2.88 & 1064 & {$[92]$} \\
$\mathrm{FS}$ & 1.45 & 2.46 & 1064 & {$[91]$} \\
$\mathrm{KTP}$ & 1.77 & 23.67 & 1064 & {$[92]$} \\
$\mathrm{MgF} 2$ & 1.4 & 0.57 & 1064 & {$[92]$} \\
$\mathrm{Te} \mathrm{Glass}$ & 2.0 & 20.95 & 1064 & {$[92]$} \\
Air & 1.0 & 0.012 & 308 & {$[93]$} \\
\hline
\end{tabular}

Tab. 4-1 Nonlinear refractive index parameters of different materials. 
The intensity dependence of $\mathrm{n}$ implies a refractive index varying in time and space causing SPM and SF respectively.

A general description of SPM is given in $[90,94]$ and therefore at this point only briefly described. As the pulse propagates through a medium with positive nonlinear refractive index the intensity at any point increases and than falls as the pulse goes past and will create a timevarying refractive index of the medium. This variation in refractive index produces a shift in the instantaneous phase of the pulse and results in a frequency shift. In other words a temporally varying phase implies that the instantaneous optical frequency differs across from its central frequency and new frequency components are continuously created as the pulse propagates through the media. The maximum frequency shift occurs at the points of maximum slope or maximum $\mathrm{dI}(\mathrm{t}) / \mathrm{dt}$.

A pulse with a smooth time envelope acquires a linear frequency chirp and increases linear with the distance while propagating through the medium, at least as long as the temporal pulse shape remains unchanged.

SPM is utilized for example in nonlinear compression schemes where additional frequency components are created to further compress pulses to shorter durations [95]. In regenerative amplifiers SPM is usually undesirable. In most optical media, group-velocity dispersion (GVD) is present and the optical frequencies travel with different group velocities resulting in a changed pulse shape and can lead to strong pulse distortions and pulse broadening.

Whereas SF is a consequence of the refracted index dependency of the light intensity in materials with a positive nonlinear refractive index $\bar{n}_{2}$. A nonuniform intensity distribution across the beam profile leads to a transverse variation of the index of refraction. For dielectric materials the refractive index decreases for a Gaussian beam from the center with increasing radial coordinate. This modified refractive index distribution acts like a focusing lens [90] and can lead to severe damage in small focused spots in an optical system.

One can define a "self trapping" power $\mathrm{P}_{\mathrm{cr}, 1}$ as the power for which the wave front curvature change caused by diffraction is exactly compensated by the change in the wave front curvature due to self-lensing over a short distance. $\mathrm{P}_{\mathrm{cr}, 1}$ is determined by

$$
\mathrm{P}_{\mathrm{cr}, 1}=\mathrm{I}_{0} \frac{\pi \mathrm{w}_{0}^{2}}{2}=\frac{\lambda_{\mathrm{s}}^{2}}{8 \pi \mathrm{n}_{0} \overline{\mathrm{n}}_{2}}
$$

where $I_{0}$ is the peak intensity, $\mathrm{w}_{0}$ the center frequency and $\lambda_{\mathrm{s}}$ the signal wavelength. For a $\beta$ barium borate $(\mathrm{BBO})$ Pockels cell as used in the regenerative amplifier described below, $\mathrm{P}_{\mathrm{cr}, 1}$ reaches $888.3 \mathrm{~kW}$ using equation $4-5, \overline{\mathrm{n}}_{2}(\mathrm{BBO})=2.88 \cdot 10^{-16} \mathrm{~cm}^{2} / \mathrm{W}$ and $\mathrm{n}_{0}(\mathrm{BBO})=1.65$.

\subsection{The B-integral}

SPM is often accompanied and enhanced by SF in the same system. To estimate the cumulated nonlinear effects occurring for example in multipass and regenerative amplifiers, it has become conventional to use the "B-integral" as a measure of the nonlinear phase accumulation of a pulse as it propagates through a material and is given by 


$$
\mathrm{B}=\frac{2 \pi}{\lambda_{\mathrm{s}}} \int_{0}^{\mathrm{L}} \overline{\mathrm{n}}_{2}(\mathrm{z}) \mathrm{I}(\mathrm{z}) \mathrm{dz}
$$

taking into account the changes in diameter and power level at each position $\mathrm{z}$ of the laser beam through the complete system of the length L. A generally accepted value for the cumulative B-integral for high-power laser is $\mathrm{B} \leq 3$ to 5 to avoid serious nonlinear damage and temporal pulse distortions caused by either SPM or SF. Noticeable spectral distortion begins at $\mathrm{B} \sim 1.5-2.0$.

Using equation 4-6 for a pulse with the duration $\tau$ in a regenerative amplifier containing a Pockels cell with the length $\mathrm{L}_{\mathrm{PC}}$ exhibiting the nonlinear refractive index $\overline{\mathrm{n}}_{2 \mathrm{PC}}$ and a gain medium with the length $L_{b}$ owning the nonlinear refractive index $\bar{n}_{2 b}$ the B-integral can be calculated for a single roundtrip starting with an initial intra cavity energy $E_{1}$ as

$$
\mathrm{B}=\frac{2 \overline{\mathrm{n}}_{2 \mathrm{PC}} \mathrm{L}_{\mathrm{PC}} \mathrm{E}_{1}}{\lambda_{\mathrm{s}} \tau_{\mathrm{PC}}{ }^{2}}+\frac{\overline{\mathrm{n}}_{2 \mathrm{~b}} \mathrm{E}_{1} \mathrm{~L}_{\mathrm{b}}(1+\mathrm{g})}{\lambda_{\mathrm{s}} \tau \mathrm{r}_{\mathrm{b}}{ }^{2}}+\frac{\overline{\mathrm{n}}_{2 \mathrm{~b}} \mathrm{gE}_{1} \mathrm{~L}_{\mathrm{b}}(1+\mathrm{g})}{\lambda_{\mathrm{s}} \tau \mathrm{r}_{\mathrm{b}}{ }^{2}}+\frac{2 \overline{\mathrm{n}}_{2 \mathrm{PC}} \mathrm{L}_{\mathrm{PC}} \mathrm{g}^{2} \mathrm{E}_{1}}{\lambda_{\mathrm{s}} \tau \mathrm{r}_{\mathrm{PC}}{ }^{2}}
$$

with the two beam diameters in the $\mathrm{PC}$ and the gain medium $\mathrm{r}_{\mathrm{PC}}$ and $\mathrm{r}_{\mathrm{b}}$ respectively. The first term contains the B-integral introduced by the pulse with the initial Energy $\mathrm{E}_{1}$. The second and third term contain the B-integral introduced by the gain medium amplifying the energy from $E_{1}$ to $g \cdot E_{1}$ and $g \cdot E_{1}$ to $g^{2} \cdot E_{1}$ respectively. The last term contributes the B-integral added by the pulse being twice amplified in the gain medium passing through the PC again.

For a regenerative amplifier based on a thin disk where the length of the disk (usually 10${ }^{1} \mathrm{~mm}$ ) is negligible compared to the crystal length of the PC the total accumulated B-integral for the total number of roundtrips $n_{\text {tot }}$ inside the resonator is given as

$$
\mathrm{B}=\sum_{\mathrm{n}=1}^{\mathrm{n}=\mathrm{n}_{\mathrm{tot}}} \frac{4 \overline{\mathrm{n}}_{2} \mathrm{~L}_{\mathrm{PC}} \mathrm{g}_{\mathrm{RT}} \mathrm{E}_{0}}{\lambda_{\mathrm{s}} \tau \mathrm{r}_{\mathrm{PC}}}
$$

where $\mathrm{E}_{0}$ is the seed energy and $\mathrm{g}_{\mathrm{RT}}$ the total roundtrip gain.

\subsection{B-Integral of the regenerative amplifier}

An electro-optical device is required to switch the pulses in and out of the cavity and has to be passed twice for each round trip inside the cavity. The PC is the only bulk material inside the cavity accumulating B-integral neglecting the thin laser disk with a few tenth of a millimeter compared to the usual length of $40 \mathrm{~mm}$ crystal in a commercially available PC. To amplify the pulses in the regenerative cavity without CPA, SPM and SF in the PC should be impeded by any chance to evade changes in the pulse spectra and beam profile causing instabilities in the beam parameters such as filamentation, temporal pulse distortion or even material damage.

Taking CPA not into account the possibilities to keep the B-integral caused by the PC low are high gain inside the regenerative cavity reducing the number of necessary round trips, large beam diameter in the PC decreasing the intensity and the use of a short PC-crystal minimizing the path length through the critical media. 
As a result of first considerations the regenerative amplifier was planned to be designed for a total output energy of $5 \mathrm{~mJ}$ at a repetition rate of $10 \mathrm{kHz}$ with pulse duration of $2 \mathrm{ps}$. For most disk based amplifiers the gain per round trip is low as the gain media is usually only one tenth of a millimeter thin and lies typically in the range between 10 and $40 \%[28,96]$.

Calculated with equation 4-8, figure 4-1 shows the accumulated B-integral of a BBO PC over the number of round trips for a given calculated gain to achieve pulse energies of $5 \mathrm{~mJ}$ starting from a seed level of $1 \mathrm{pJ}$. Three different conditions were calculated using pulse durations of 2 and $10 \mathrm{ps}$, PC crystal length of 20 and $40 \mathrm{~mm}$ and beam radii of $1.5,2.0$ and $3.0 \mathrm{~mm}$. With an expected gain of $20 \%$ roughly 80 roundtrips are required to reach pulse energies of $5 \mathrm{~mJ}$. Aiming for $5 \mathrm{~mJ}$ pulses with durations of 2 ps without CPA figure 4-1 shows that only a large beam diameter of $6 \mathrm{~mm}$ in a $20 \mathrm{~mm}$ short PC-crystal can keep the B-integral small enough (green graph) to avoid a large B-integral for a reasonable number of round trips somewhere between 40 and 100. In this case the calculated B-integral reaches a still acceptable value of 2.57. If the beam diameter is reduced to $2 \mathrm{~mm}$ the B-integral already reaches a critical value of 5.77. Only stretching the pulse for example to $10 \mathrm{ps}$ would decrease the Bintegral significantly for smaller beam diameters.

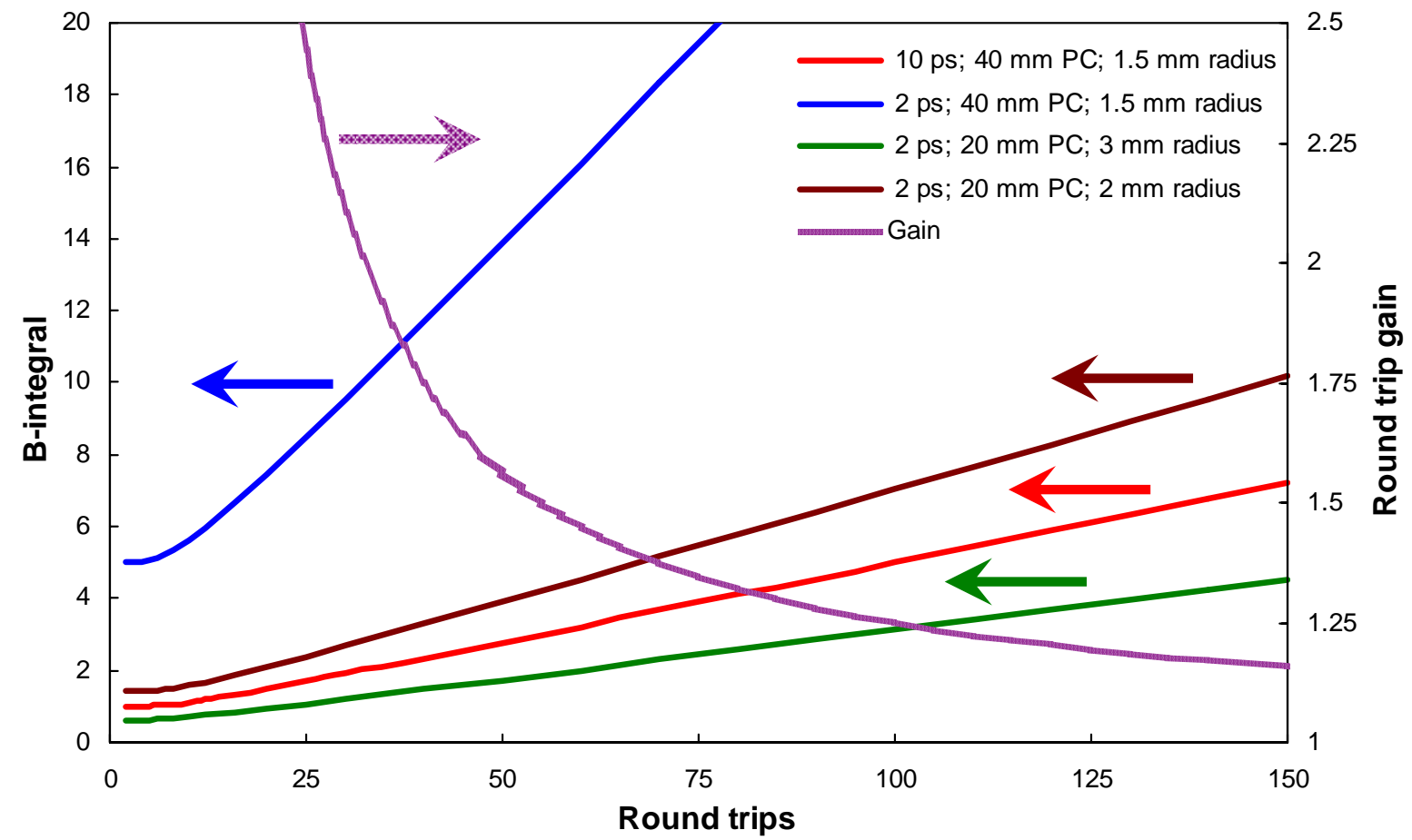

Fig. 4-1 Calculated B-integral for different pulse durations, PC length and beam diameters in dependence of the amount of round trips at a given gain to reach an energy level of $5 \mathrm{~mJ}$ starting from a seed energy of 1 pJ.

Later, it was found that the achievable energy directly out of the regenerative amplifier can reach more than $30 \mathrm{~mJ}$ and required a CPA setup to avoid SPM. Figure 4-2 shows the calculated accumulated B-integral in a $20 \mathrm{~mm}$ long PC for out coupled pulse energies of $32 \mathrm{~mJ}$, a beam diameter of $2.5 \mathrm{~mm}$ (radius $1.25 \mathrm{~mm}$ ) and three different pulse durations of $20 \mathrm{ps}, 70 \mathrm{ps}$ and 200 ps respectively. 


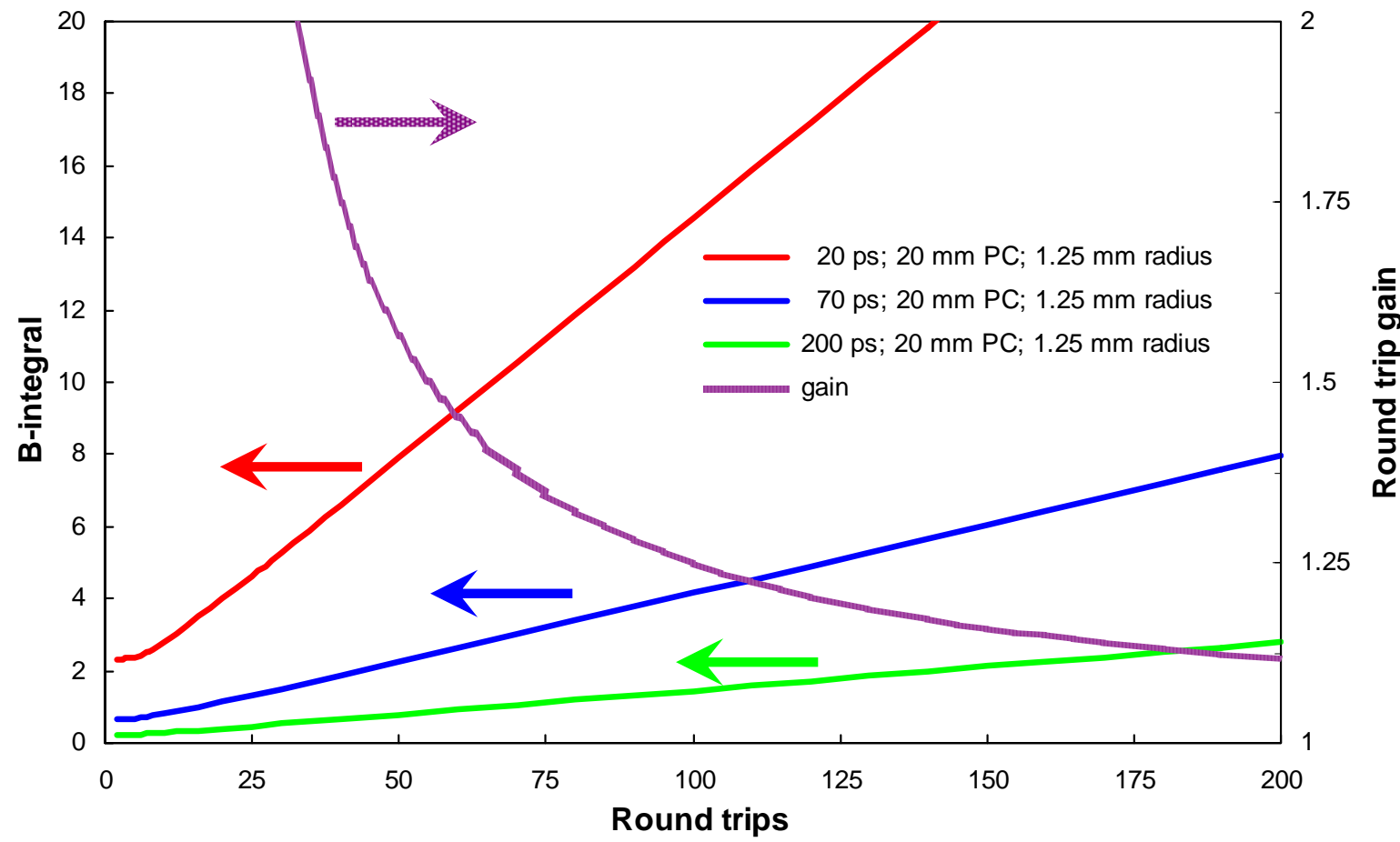

Fig. 4-2 Calculated B-integral for a PC length of $20 \mathrm{~mm}$, a beam diameter of $2.5 \mathrm{~mm}$ (radius $1.25 \mathrm{~mm}$ ) and three different pulse durations of $20 \mathrm{ps,} 70 \mathrm{ps}$ and $200 \mathrm{ps}$ respectively.

\subsection{The Pockels cell}

For coupling the pulses in and out of the amplifier resonator a BBO-crystal containing PC as electro-optic switching device was chosen. PCs are well described in [37,97] and not further explained in detail at this point. Applied to high-voltage (HV) pulses, piezoelectric effects can give rise to acoustic waves in nonlinear crystals which can ruin the performance of the Pockels cell by modulating the crystal birefringence through the elastooptic effect long after the high-voltage pulse is applied. Effective Q-switching of lasers at high repetition rate implies that the crystal is relatively free of piezoelectric ringing. BBO Q-switches have been reported to exhibit negligible piezoelectric ringing at repetition rates of up to $6 \mathrm{kHz}$. Only a small acoustic contribution around $5 \%$ to the total electro-optic coefficient has been measured [98] explaining why no piezoelectric ringing is detected with BBO Pockels cells up to $6 \mathrm{kHz}$ [99]. The combination of high optical damage threshold, (single-shot bulk damage threshold value, up to $50 \mathrm{GW} / \mathrm{cm}^{2}$ at $1064 \mathrm{~nm}$ [100]) reduced piezoelectric ringing and relatively low insertion loss makes this crystal particularly attractive for electro-optic Q-switching of high peak and average power solid-state lasers [96].

A disadvantage of $\mathrm{BBO}$ is the relatively high quarter-wave voltage $\mathrm{V}_{1 / 4}$ due to the low electro-optic coefficient $\mathrm{r}_{22}^{\mathrm{T}}$ of $2.2 \mathrm{pm} / \mathrm{V}$. The required voltage to turn the polarization 45 degree in a crystal with the length $L$ and the thickness $d$ can be calculated as

$$
\mathrm{V}_{1 / 4}=\frac{\lambda_{\mathrm{s}} \mathrm{d}}{\mathrm{n}_{0}^{3} \mathrm{r}_{22}^{\mathrm{T}} \mathrm{L}}
$$


To meet the requirements of the estimated beam diameter of $6 \mathrm{~mm}$ in the PC considered in chapter 4-2 an aperture of $12 \mathrm{~mm}$ and a crystal length of $20 \mathrm{~mm}$ seem to be appropriate and result in a quarter-wave voltage in the range of $16 \mathrm{kV}$. PCs and fast switches for such HV were at the beginning of the regenerative amplifier project not commercially available and had to be developed first.

The electronics (driver, HV switch) were developed by the company Bergmann Messgeräte Entwicklung $\mathrm{KG}$ and the crystal holder was designed as part of this work. Figure 4-3 shows the PC-crystal in the water cooled ceramic $\left(\mathrm{Al}_{2} \mathrm{O}_{3}\right)$ holder between two copper electrodes. The center picture shows the $\mathrm{PC}$ in operation with some green light through parasitic second harmonic generation in the $\mathrm{BBO}$ crystal. On the right side the $\mathrm{PC}$ and the water cooled $\mathrm{HV}$ switch are displayed together.

The optical switching time of the developed device was measured with a photodiode (rise time $<175 \mathrm{ps})$ and a cw-laser. Therefore an infrared alignment laser $\left(\lambda_{\mathrm{s}}=1047 \mathrm{~nm}\right.$; $\mathrm{P}_{\mathrm{cw}}=100 \mathrm{~mW}$ ) was sent through a polarizer, the PC and reflected the same way back with an high reflecting mirror. Through switching the HV resulting in a 90 degree turn of the polarization after the double pass through the PC crystal, parts of the cw signal could be sliced out via the polarizer and measured with the photo diode. Figure 4-4 shows the time duration taking the PC to switch on and off the $16 \mathrm{kV}$ quarter-wave voltage. The switching time was estimated from figure $4-4$ to be $\sim 12$ ns.
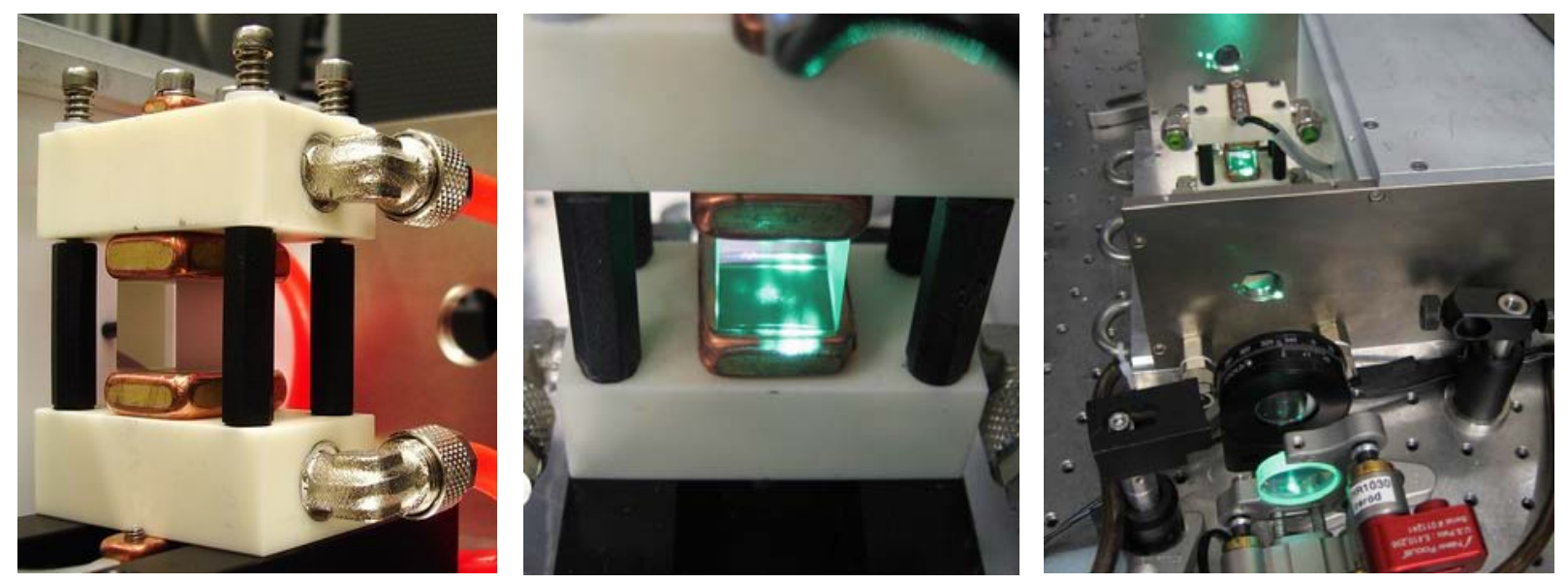

Fig. 4-3 The PC-crystal in the water cooled ceramic $\left(\mathrm{Al}_{2} \mathrm{O}_{3}\right)$ holder between two copper electrodes. The center picture shows the $\mathrm{PC}$ in operation with some green light through parasitic second harmonic generation in the $\mathrm{BBO}$ crystal. On the right side the $\mathrm{PC}$ is displayed together with the water cooled HV-switch.

The time required to switch the PC needs to be faster than the roundtrip time of the regenerative amplifier. Otherwise some part of the pulse would not be completely injected and even worse not properly ejected out of the resonator after an amplification cycle with several round trips and would remain one more round trip inside the amplifier. Figure 4-6 shows two full recorded switching cycles of the PC for a repetition rate of $10 \mathrm{kHz}$. 


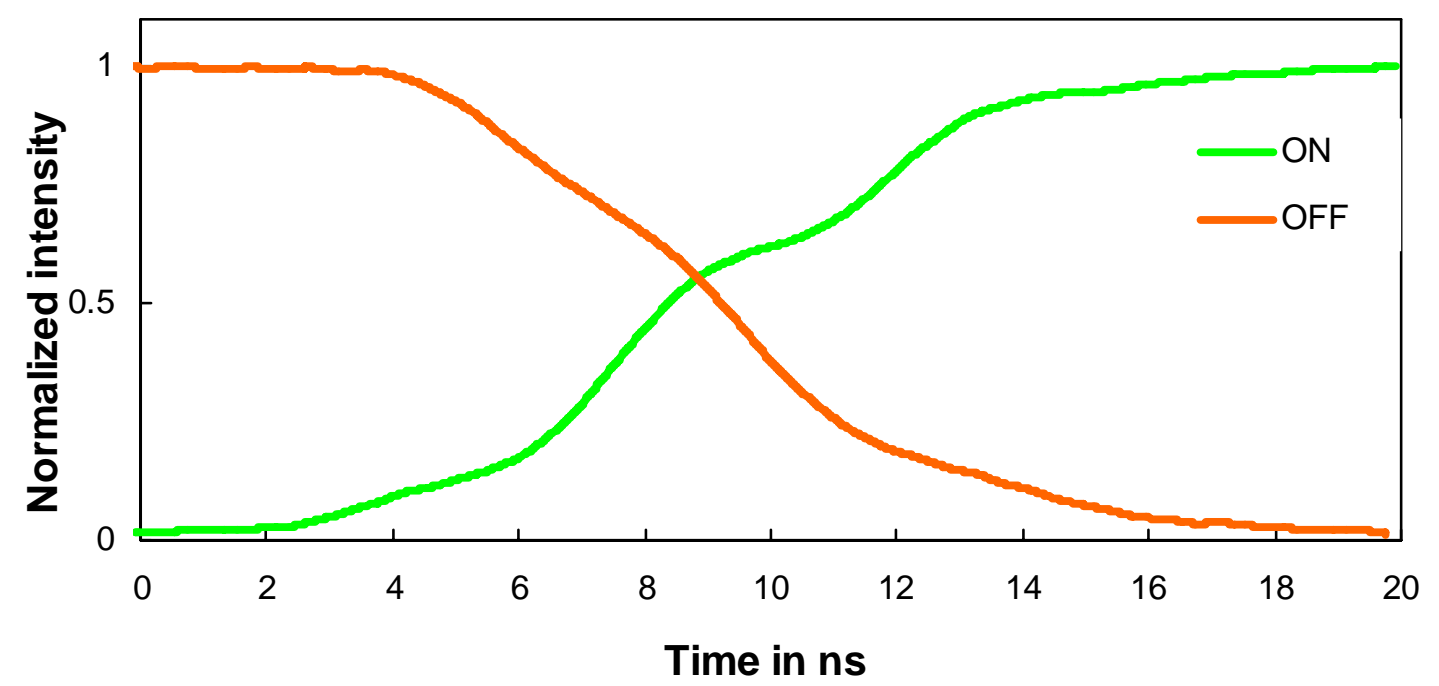

Fig. 4-4 Switching time of the PC. The switching time was measured to be $\sim 12$ ns. Short enough for switching being well below the round trip time of the regenerative amplifier.

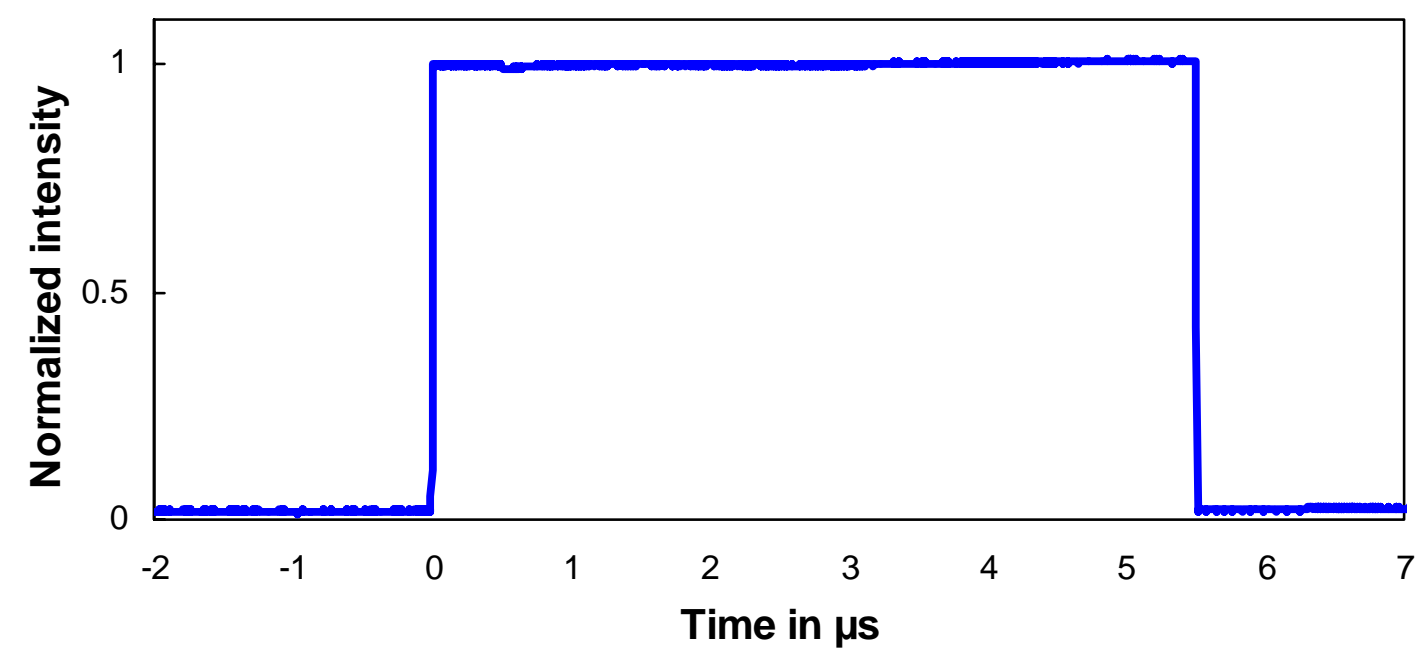

Fig. 4-5 Sliced out signal for a switching time of $5.5 \mu \mathrm{s}$. No significant ringing was observed at a repetition rate of $10 \mathrm{kHz}$ while the $\mathrm{HV}$ was applied on the crystal.

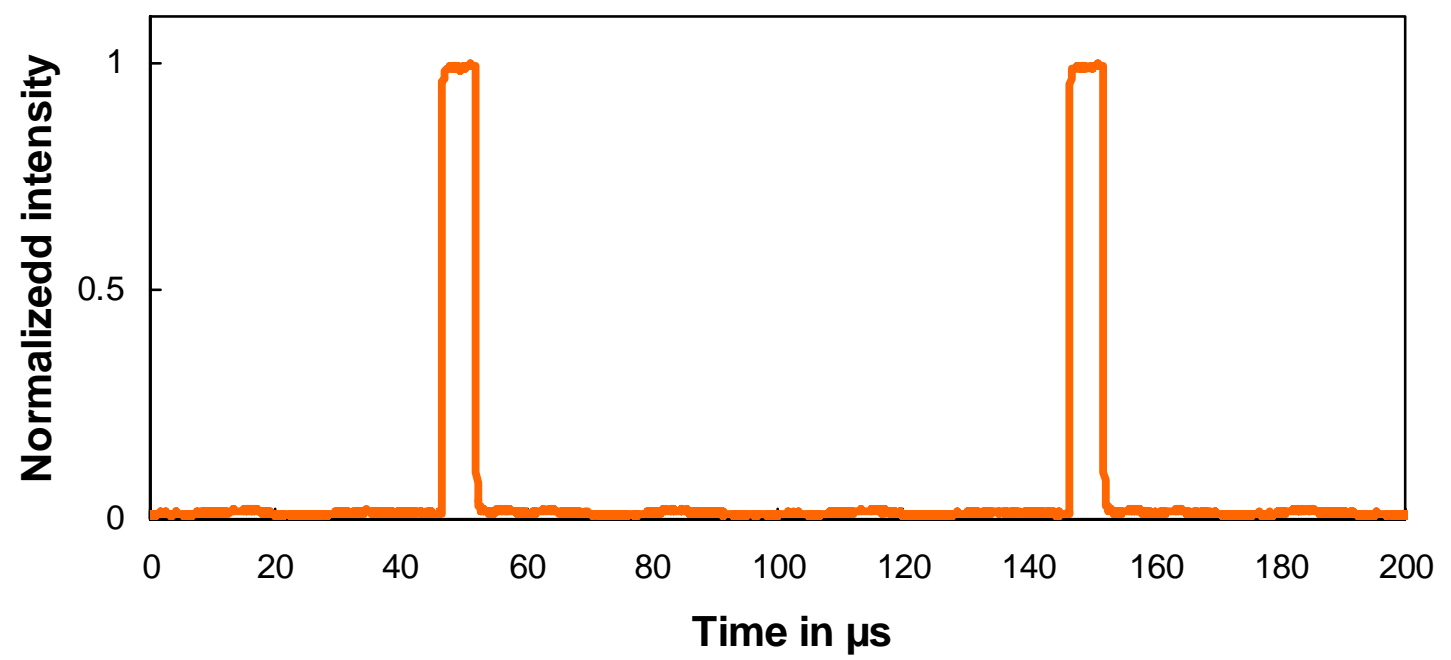

Fig. 4-6 Two full recorded $5.5 \mu$ s long switching cycles of the PC at an applied repetition rate of $10 \mathrm{kHz}$. 


\section{Regenerative amplifier without chirped pulses based on the IFSW disk laser head}

The first regenerative amplifier was built on base of the thin disk laser head designed and developed at the Institut für Strahlwerkzeuge (IFSW) at the Technical University Stuttgart seeded by the $\mathrm{Yb}: \mathrm{KGW}$ oscillator described in chapter 3.1. The amplifier head from the IFSW shown in figure 5-1 contains a $\sim 200 \mu \mathrm{m}$ thick and $\sim 0.1$ degree wedged Yb:YAG thin-disk with a doping level of 7\% Ytterbium mounted on a heat sink water cooled from the back side, and pumped via fiber coupled diodes with up to $500 \mathrm{~W}$ at a wavelength of $940 \mathrm{~nm}$. Special pump optics guide the pump light (see chapter 3.1) after the fiber exit 16 times through the thin disk and is thereby almost completely absorbed in the laser crystal. Coated on the front side with an anti-reflex coating for the pump light at $940 \mathrm{~nm}$ and the laser radiation of $1030 \mathrm{~nm}$, the backside is coated for both wavelengths with an high reflecting coating and soldered to a cooling plate as shown in figure 5-2.

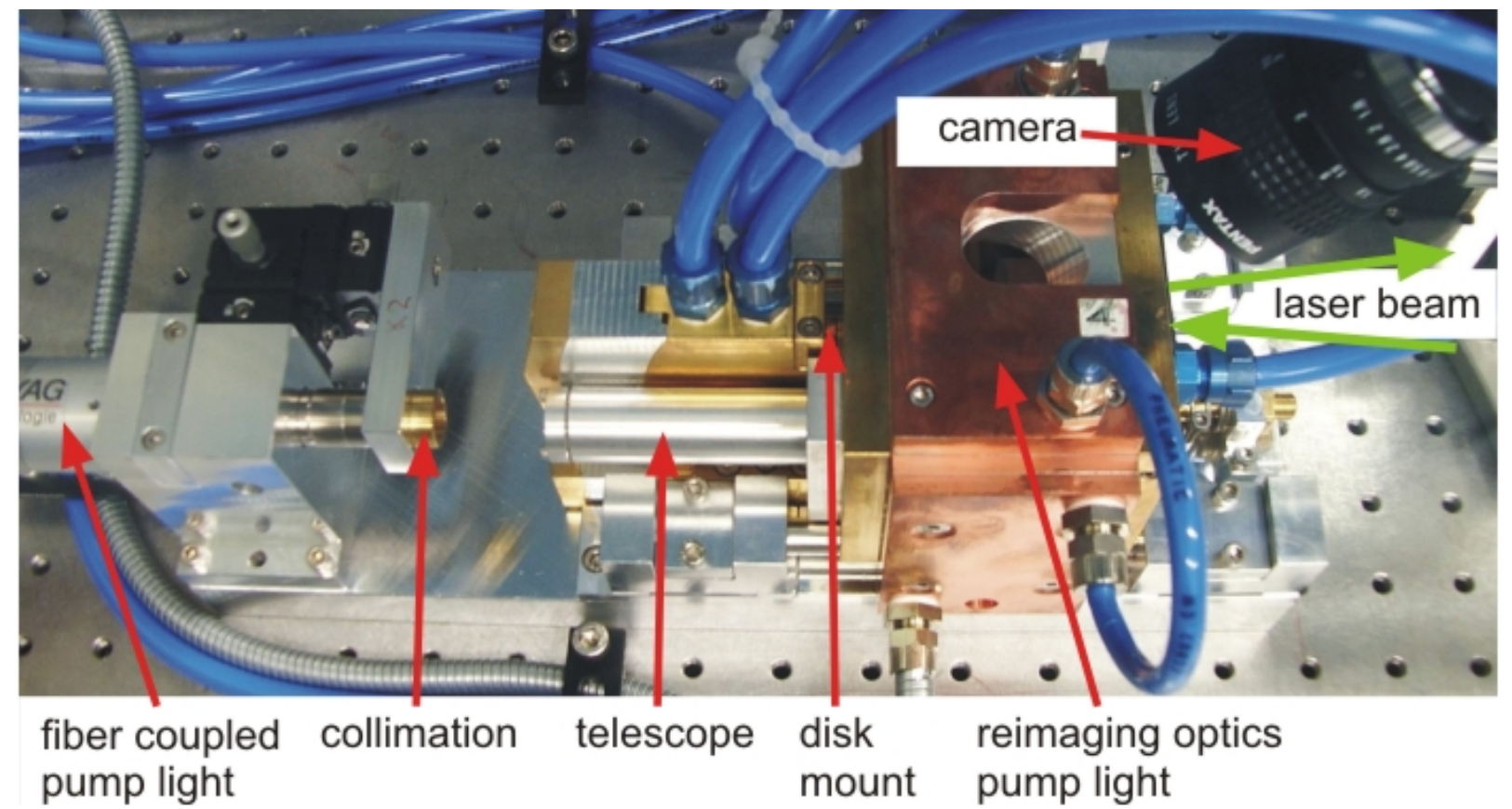

Fig. 5-1 Disk based amplifier head from the Institut für Strahlwerkzeuge (IFSW) at the Technical University Stuttgart.

Wedging the disk with $\sim 0.1$ degree is required since a plane parallel disk inside the cavity would act due to interference between the two parallel disk faces caused by imperfect antireflex coating as a gain narrowing etalon leading to shorter pulse durations. Furthermore, a wedged disk ejects reflections from the surface of the disk out of the cavity. In case of a plane parallel disk the reflections would stay inside the cavity being amplified while traveling with the main pulse and would degrade the temporal pulse shape and is described in chapter 6.3.1. Additionally the edge of the round Yb:YAG disk is grinded down as shown on the left side in figure 5-2 to avoid parasitic lasing via the side surfaces. Figure 5-2 shows the disk mounted on the cooling plate at the left side and the pumped disk inside the amplifier head at the right side. To prevent optical damage on the laser disk a beam diameter of $3.2 \mathrm{~mm}$ was chosen to- 
gether with a pump spot size of $4 \mathrm{~mm}$. The beam diameter should be around $80 \%$ of the pump spot in order to avoid the outer pumped area where a heavy phase step between the pumped and the unpumped area occurs inducing a poor beam quality. Concerning the abovementioned ancillary conditions the cavity design in figure 5-3 was developed considering the given radius of curvature of $5 \mathrm{~m}$ of the disk.
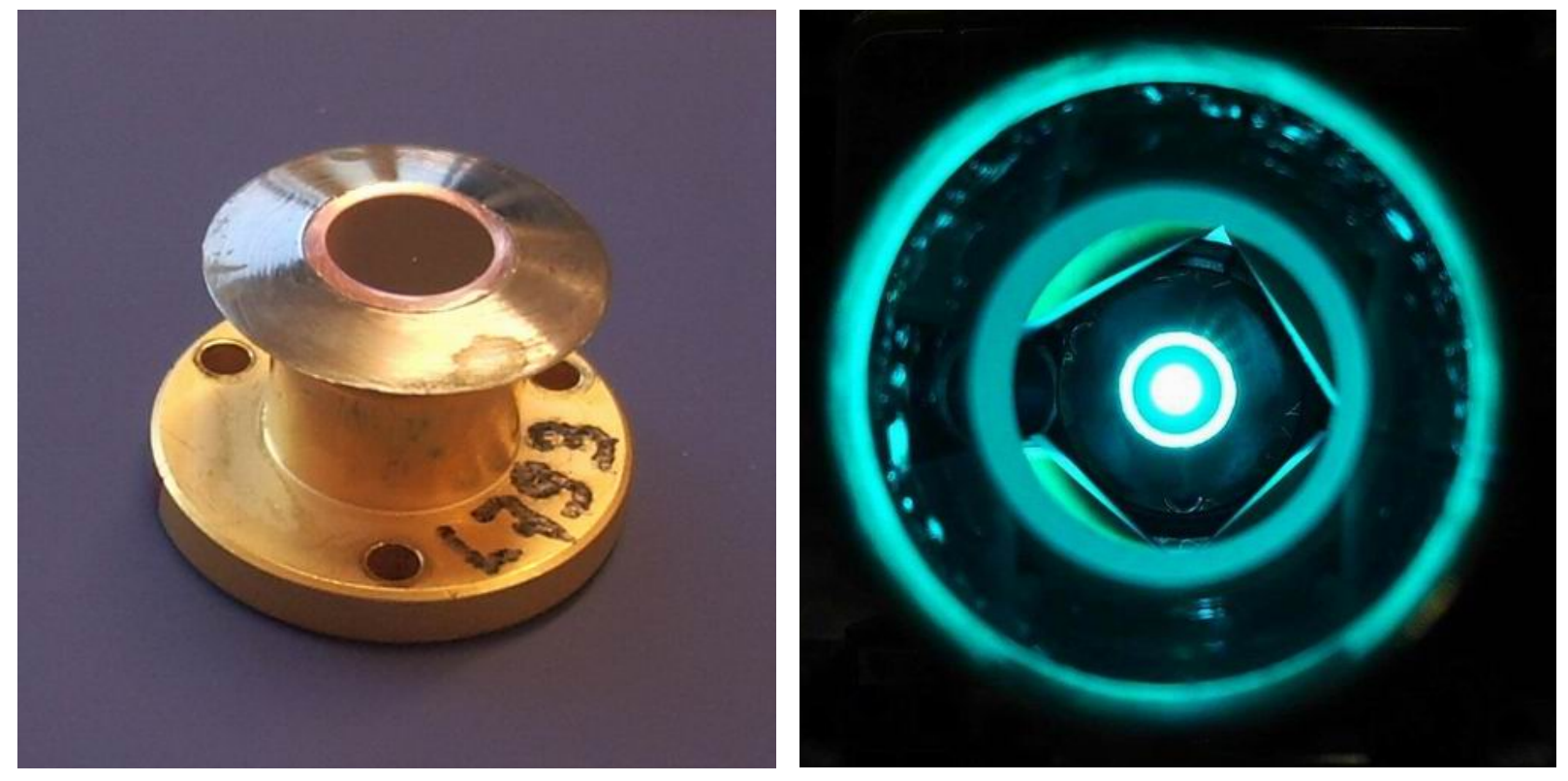

Fig. 5-2 Mounted disk on the cooling plate (left side) and pumped disk inside the amplifier head (right side). The edge of the disk is grinded down to avoid parasitic lasing via the side surfaces. Impurities in the disk lead to green fluorescence while pumping.

For modeling the cavity the software Paraxia and WinLase were used. Concerning the cavity design the following terms had to be considered and are summarized as follows:

- large beam diameter in the PC $\sim 6 \mathrm{~mm}$ to avoid SF and SPM

- collimated beam at the PC position for clean switching and coupling out a collimated beam after the amplification.

- large beam diameter on the disk of $3.2 \mathrm{~mm}$ following the recommendation of the IFSW to avoid optical damage of the disk leading to a pump spot size of $4 \mathrm{~mm}$ diameter

- the radius of curvature of the disk (5 m)

- to achieve large gain the disk should be passed twice per direction inside the cavity via $2 \mathrm{f}$-imaging of the disk back to itself allowing 4 double passes through the disk per round trip

- large beam diameters on all other optics to avoid optical damage

Figure 5-3 shows the modeled cavity design together with the exact data of the used mirrors and distances in table 3-1. The complex cavity design with rather large beam diameters on the disk and at the position of the PC supports only the $\mathrm{TEM}_{00}$ fundamental laser mode leading on the one hand to a nearly diffraction-limited focused output beam of significantly improved spatial properties compared to the seed but on the other hand to an extremely sensitive behav- 
ior with respect to the thermal lens on the disk. Already small changes of the pump power in the range of $\sim 10 \mathrm{~W}$ were leading to a completely instable cavity not being able to amplify the seed pulses and maintaining the same spatial properties of the pulses inside the amplifier cavity from roundtrip to roundtrip.

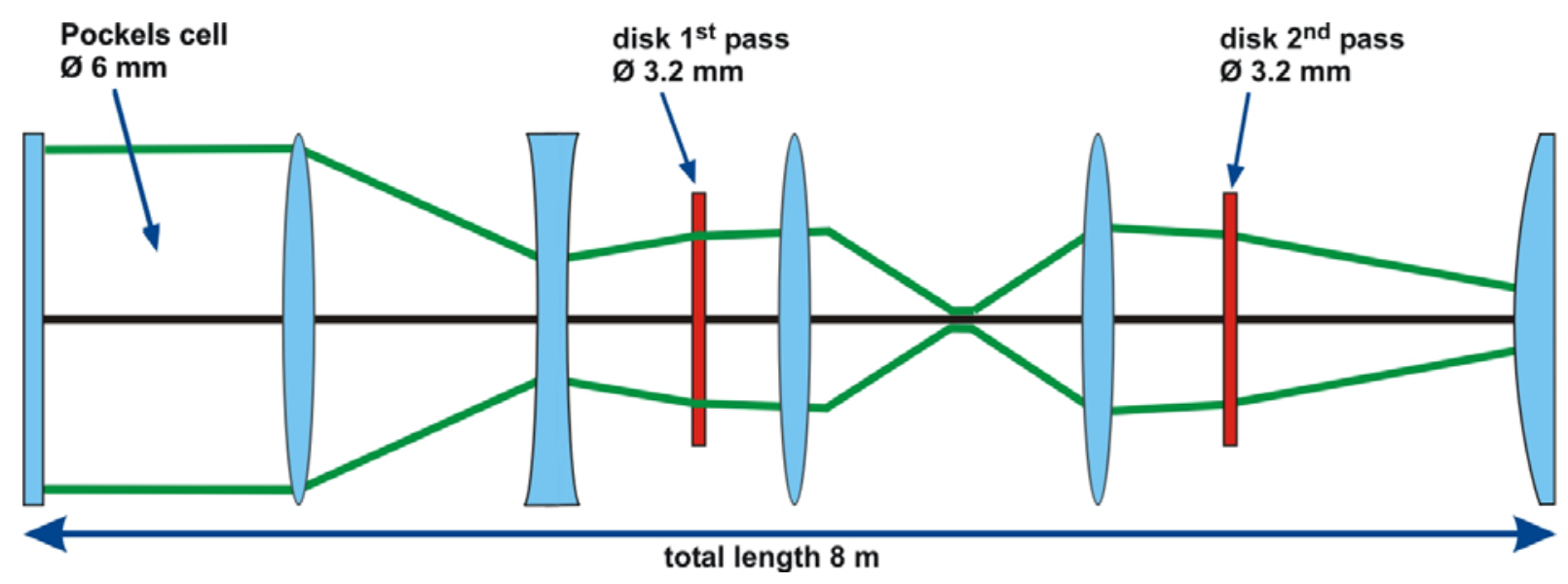

Fig. 5-3 Cavity design of the disk based regenerative amplifier using a double pass on the disk.

\begin{tabular}{c|c}
\hline mirror / distance / disk & radius of curvature / distance in mm \\
\hline end mirror & $\infty$ \\
distance & 1065 \\
mirror concave & -3000 \\
distance & 1071 \\
mirror convex & +750 \\
distance & 1680 \\
disk concave & -5000 \\
distance & 750 \\
mirror concave & -1500 \\
distance & 1463 \\
mirror concave & -1500 \\
distance & 750 \\
disk concave & -5000 \\
distance & 943 \\
end mirror convex & +1500 \\
\hline
\end{tabular}

Tab. 5-1 Distances and radii of used mirrors in the designed cavity of figure 5-3 listed from left to right.

The cavity design could be adapted to a certain thermal lens of the disk by changing the length of the telescope inside the cavity imaging the beam size of the disk back to itself. With this method, a stable cavity for a desired pump power could be designed hazarding the consequences of a smaller beam diameter around $\sim 4 \mathrm{~mm}$ at the position of the PC leading immediately to SPM at higher pulse energies. The very fact that the disk itself was absorbing laser light made the cavity optimization nearly impossible because a cavity optimized for cw could be completely instable for the laser in the pulsed mode just because of the fact that the differ- 
ence in the average power traveling inside the cavity leading to a different thermal lens. The variation of the thermal lens for an unpumped disk, pumped with $500 \mathrm{~W}$ not lasing and the same disk lasing at $100 \mathrm{~W}$ cw also pumped with $500 \mathrm{~W}$ is diagramed in figure 5-4. The thermal lens was measured with a collimated HeNe laser sent via the disk. The final position of the thermal lens was determined at the position with the smallest beam diameter of the $\mathrm{HeNe}$ laser measured with a camera.

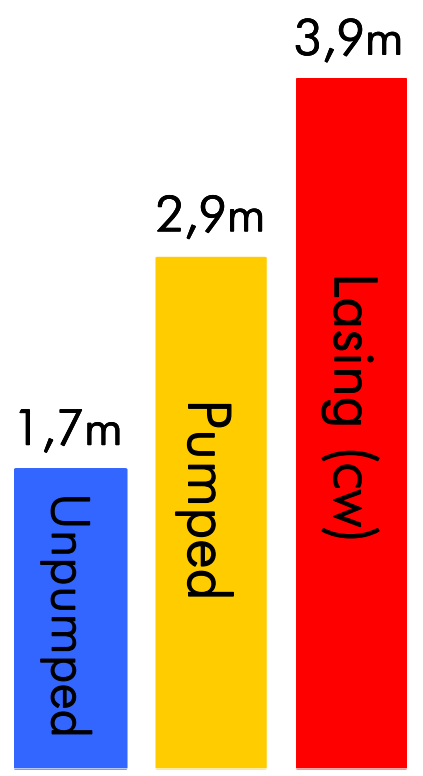

Fig. 5-4 Thermal properties of the unpumped Yb:YAG disk (blue) compared to the disk in the non lasing cavity pumped with $500 \mathrm{~W}$ (yellow) and lasing in $\mathrm{cw}$ with $100 \mathrm{~W}$ at a pump power of $500 \mathrm{~W}$ (red).

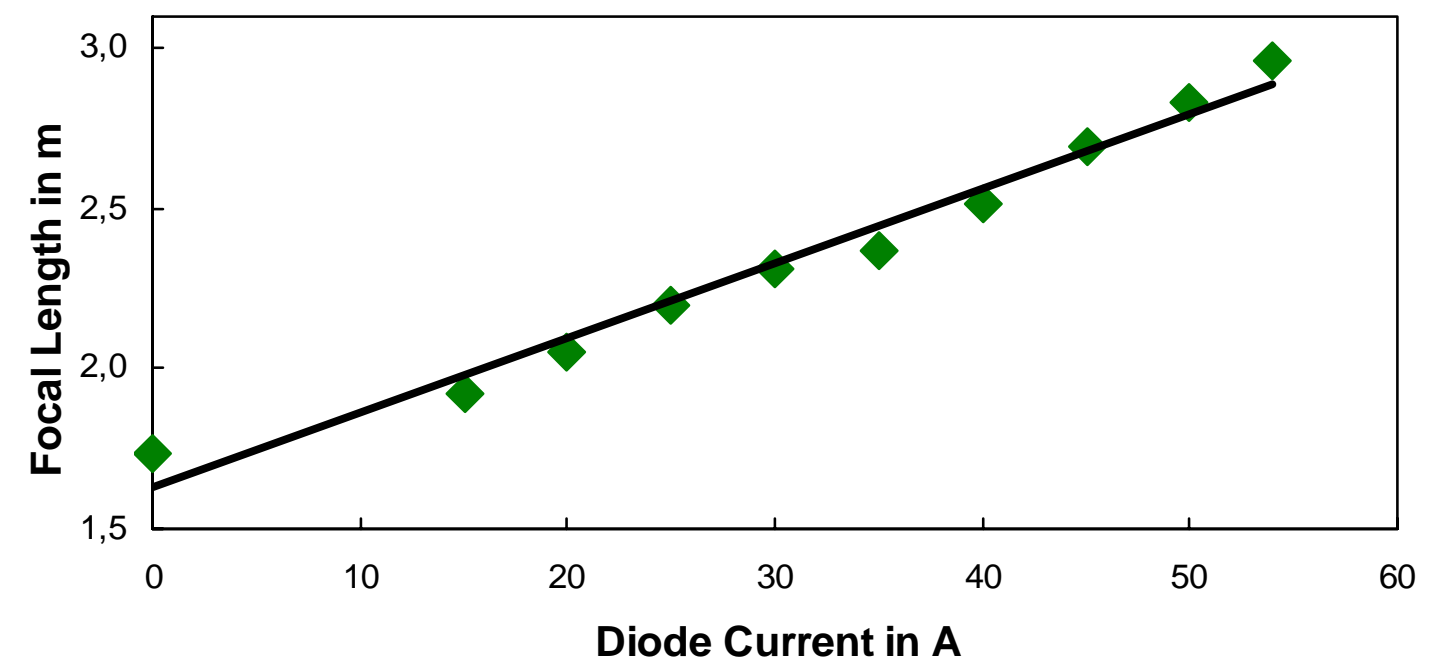

Fig. 5-5 Change of the thermal induced lens of the Yb:YAG disk in dependence of the absorbed pump radiation $(10 \mathrm{~A} \sim 100 \mathrm{~W})$.

The thermal lens of the Yb:YAG disk in dependence of the pump power is shown in figure 55. Due to heat expansion in the pumped area the disk which is from begin on bended flatens out and the radius of curvature increases linearly for the unpumped disk from $3.4 \mathrm{~m}$ to $6.0 \mathrm{~m}$ for the disk pumped with $500 \mathrm{~W}$. During the measurement of the thermal lens the cavity was blocked and no lasing occurred. 
The complete system consists of a seed laser, pulse picker, mode matching telescope and amplifier resonator. The setup is shown schematically in figure 5-6. A rubidium titanyl phosphate (RTP) Pockels cell is used to slice pulses from the $\sim 70 \mathrm{MHz}$ oscillator pulse train at a frequency of $6 \mathrm{kHz}$. An isolator protects the RTP PC against feedback from the regenerative amplifier. To avoid nonlinear effects as mentioned before, a PC based on a relatively short 20 $\mathrm{mm} \beta$-barium borate (BBO) crystal with a large aperture of $12 \mathrm{~mm}$, accepting the high quarter-wave voltage of $16 \mathrm{kV}$ has been chosen for switching the selected pulses in and out of the amplifier cavity. The current chosen beam diameter in the PC is between 4 and $6 \mathrm{~mm}$ leading to a calculated B-integral between the already critical borderline values between 3 and 6 . Inside the regenerative amplifier cavity, pulses are amplified in an arrangement similar to that described in chapter 1.1 and comparable with the thin disk based regenerative amplifier in $[29,101]$.

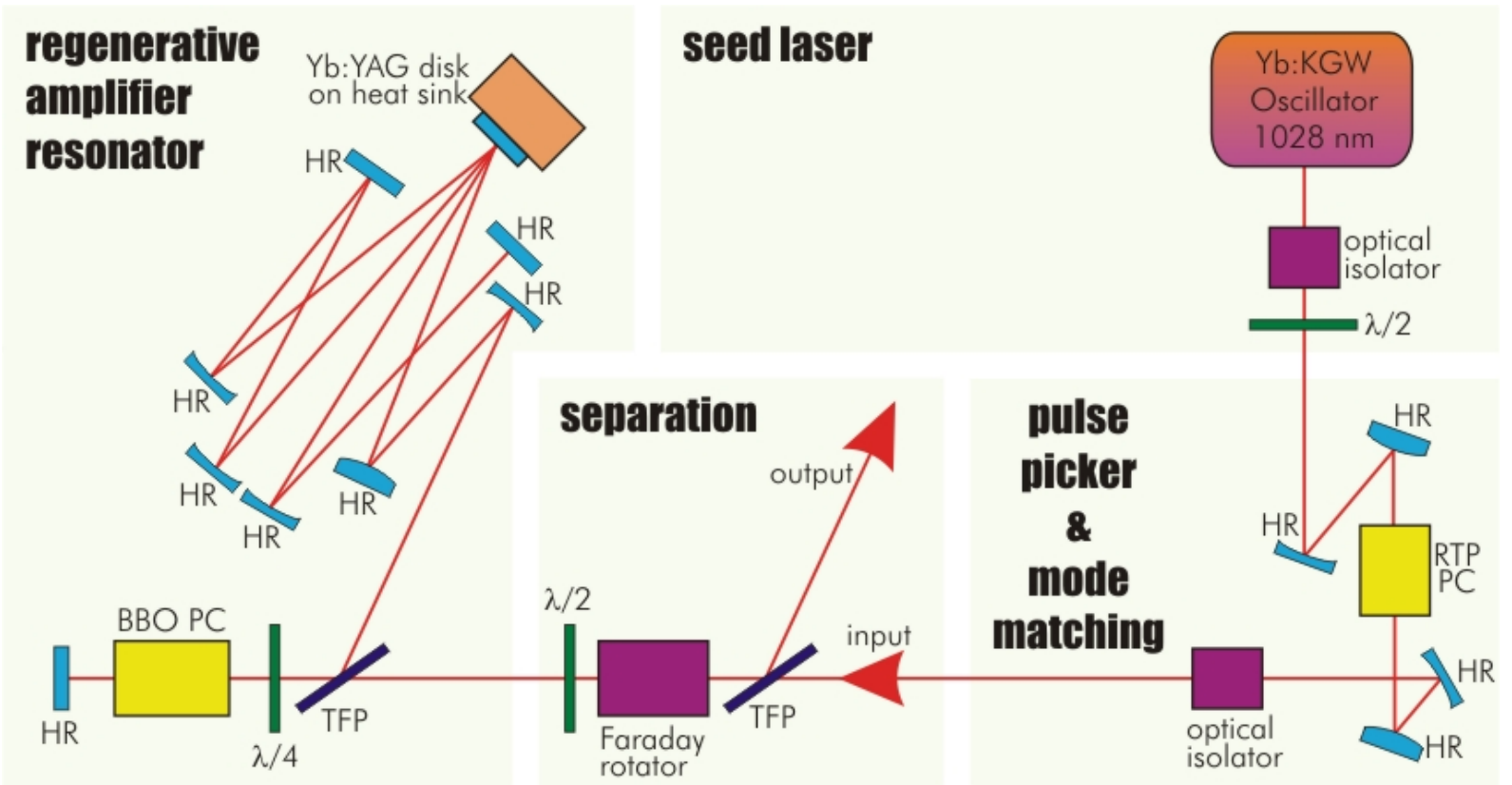

Fig. 5-6 Schematical regenerative amplifier setup. HR, high reflecting mirror; CM, chirped mirror; PC, Pockels cell; TFP, thin-film polarizer; $\lambda / 2$, half-wave plate; $\lambda / 4$, quarter-wave plate.

The seed pulses could be amplified in the regenerative amplifier at a repetition rate of $10 \mathrm{kHz}$ up to an energy level of 6.5 mJ. Figure 5-7 shows the pulse build-up inside the amplifier cavity together with the out-coupled pulse at an energy level of $4.0 \mathrm{~mJ}$. The measured resonator internal pulse build up was derived from a leakage of an intracavity folding mirror. The total round trip gain was measured from the pulse build-up to be $\sim 1.3$ for an entire round trip. As expected from the cavity design, the out coupled beam profile was close to the $\mathrm{TEM}_{00}$ fundamental laser mode and is shown in figure 5-8 at the position of the PC for the out coupled amplified beam with $4.0 \mathrm{~mJ}$ and a beam diameter of $3.7 \mathrm{~mm}$. Inside the regenerative amplifier cavity optimized for $500 \mathrm{~W}$ of pump power the pulses could be amplified to energies up to $6.5 \mathrm{~mJ}$ at a repetition rate of $10 \mathrm{kHz}$ but the small beam diameter of $3.7 \mathrm{~mm}$ in the PC caused heavy SPM and led to an unacceptable temporal pulse shape. Figure 5-9 illustrates the output spectra of the regenerative amplifier. Due to the maximum gain of the regenerative amplifier at $1030 \mathrm{~nm}$ the spectral peak shifts from the center wavelength of the $\mathrm{Yb}: \mathrm{KGW}$ oscillator at 
$1028 \mathrm{~nm}$ to $1030 \mathrm{~nm}$ after 100 roundtrips but already at pulse energies of $0.5 \mathrm{~mJ}$ spectral broadening caused by SPM occurs and broadens the bottom of the curve. Clearly visible is the spectral broadening at higher energies for example at $1.7 \mathrm{~mJ}$ and $3.7 \mathrm{~mJ}$ compared to the seed spectra.

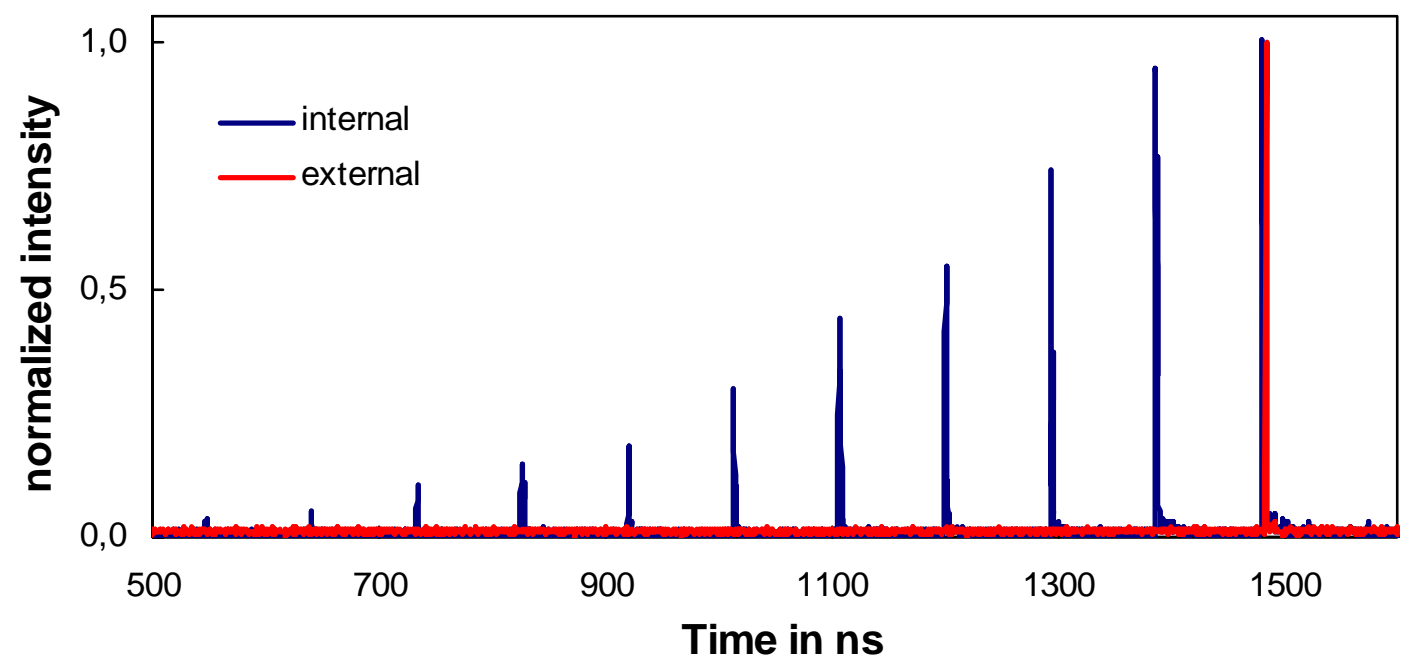

Fig. 5-7 Pulse build up in the regenerative amplifier resonator (blue) and the to the energy of $4.0 \mathrm{~mJ}$ amplified and out coupled pulse (red).The measured resonator internal pulse build-up was derived from a leakage of an intracavity folding mirror.

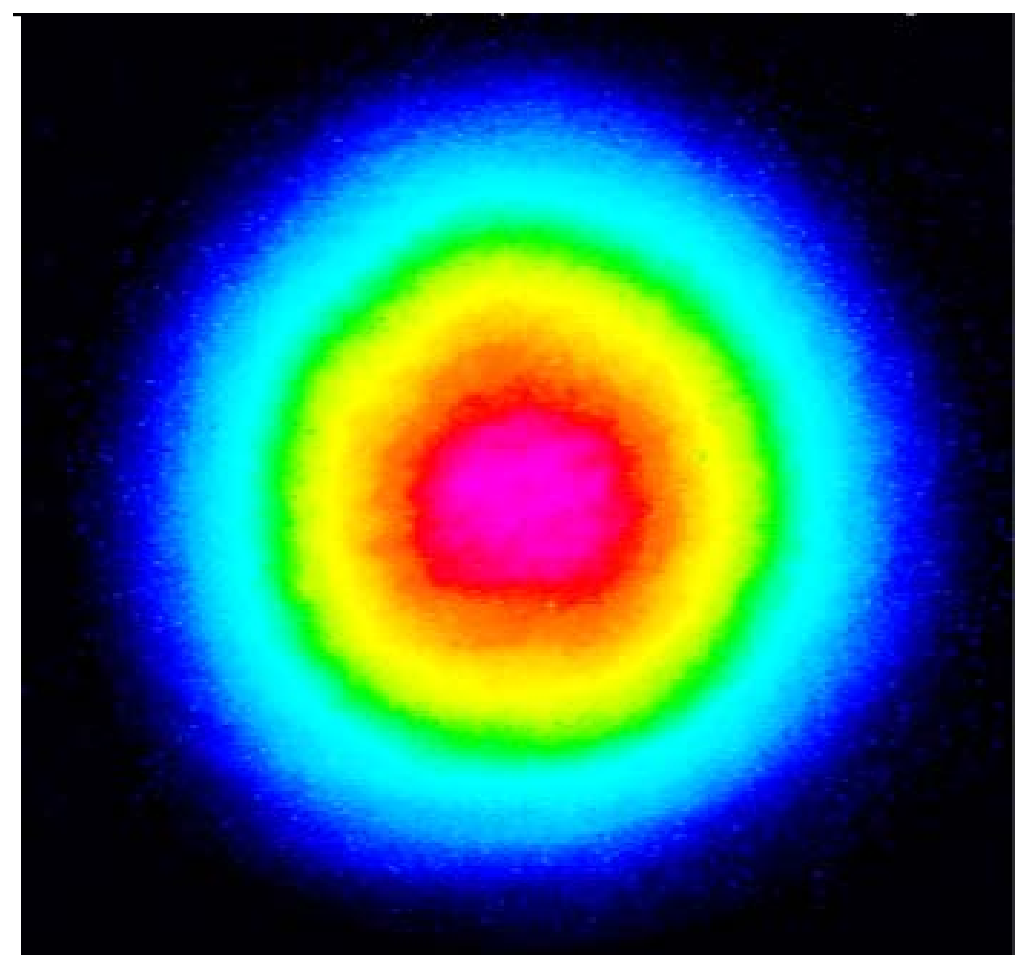

Fig. 5-8 Measured beam profile for an out coupled pulse after amplifying it to $4.0 \mathrm{~mJ}$, measured at the position of the Pockels cell with a beam diameter of $3.7 \mathrm{~mm}$.

Figure 5-10 and figure 5-11 show the measured second harmonic autocorrelation traces of the amplified pulses measured at energies of $1.7 \mathrm{~mJ}$ and $6.0 \mathrm{~mJ}$. At the energy level of $6.5 \mathrm{~mJ}$ the regenerative amplifier reached saturation caused by the creation of a broader spectra via SPM than the bandwidth of the Yb:YAG $(\sim 10 \mathrm{~nm})$ disk is able to amplify. Although an average 
output power of $65 \mathrm{~W}$ and pulses with energies of $6.5 \mathrm{~mJ}$ could be achieved, SPM caused heavy temporal distorted pulses and led to instable performance of the entire system. Caused by the rather complex amplifier resonator with large beam diameters on the disk and in the PC the pump power could be varied only over the small range of several tens of watts. A stable performance of the system could never be observed even with different cavity designs. Only amplifier resonators with smaller beam diameters in the area of the PC around $3 \mathrm{~mm}$ or smaller tend to be more stable for lower pulse energies but heavy SPM led to unacceptable pulse shapes. Being not limited by SPM the system had to be changed to a chirped pulse amplifier (CPA) [102] scheme. The further developed system based on CPA with pulse stretcher, amplifier and recompression is described in the following chapters.

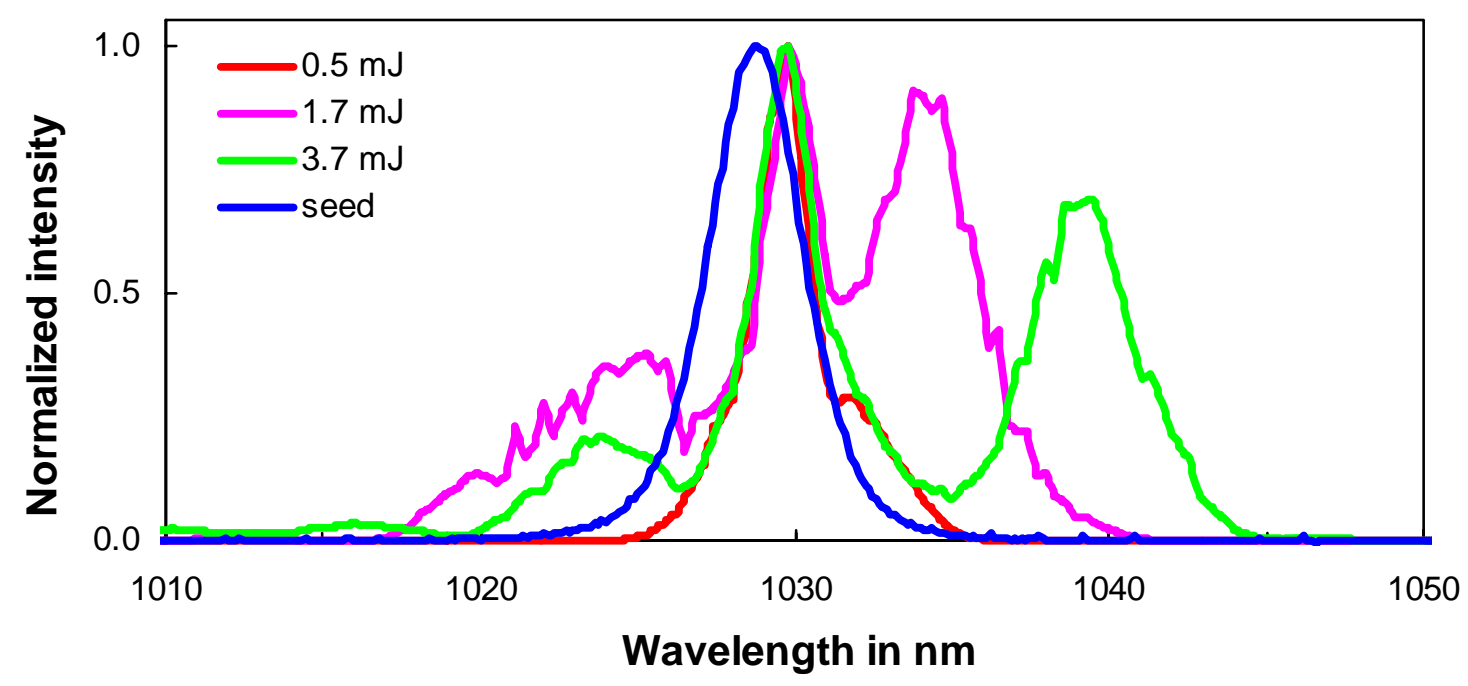

Fig. 5-9 Spectral broadening due to self phase modulation in the Pockels cell at different energy levels compared to the seed spectra. Due to the maximum gain of the regenerative amplifier at $1030 \mathrm{~nm}$ the spectral peak shifts to $1030 \mathrm{~nm}$ after 100 roundtrips. Already at pulse energies of $0.5 \mathrm{~mJ}$ spectral broadening caused by self phase modulation occurs and broadens the bottom of the curve. Clearly visible is the spectral broadening at higher energies for example at $1.7 \mathrm{~mJ}$ and $3.7 \mathrm{~mJ}$.

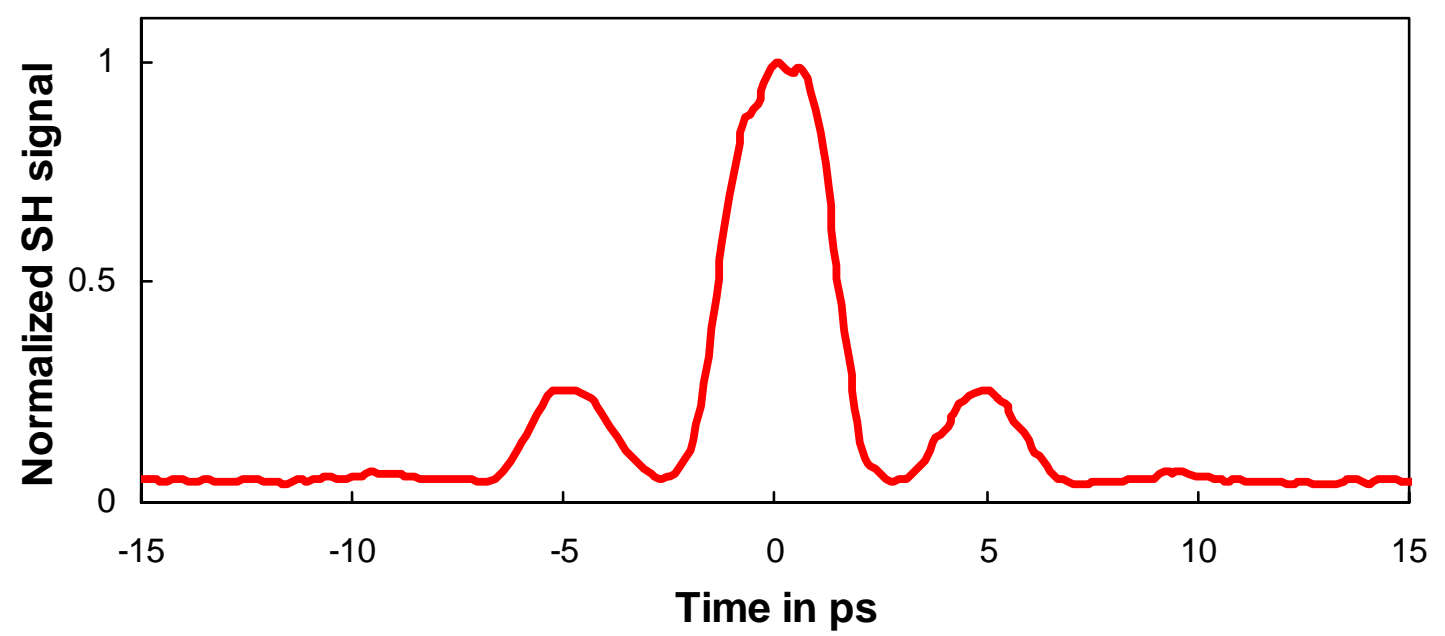

Fig. 5-10 Second harmonic autocorrelation trace of the output pulse of the regenerative amplifier at an energy level of $1.7 \mathrm{~mJ}$ achieved by 100 roundtrips and a pump power of $233 \mathrm{~W}$. 


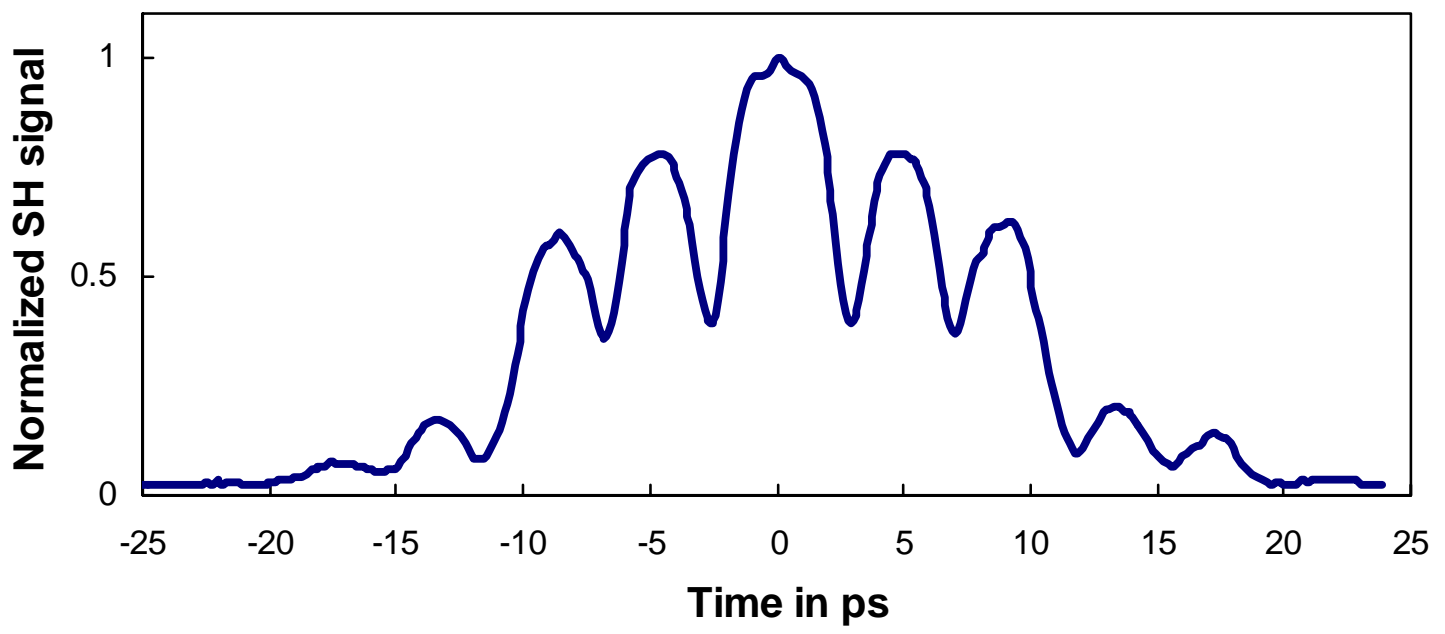

Fig. 5-11 Second harmonic autocorrelation trace of the output pulse of the regenerative amplifier at an energy level of $6.5 \mathrm{~mJ}$ achieved by 100 roundtrips and a pump power of $500 \mathrm{~W}$. 


\section{Chirped pulse amplification}

The chirped pulse amplification (CPA) technique was developed [102] to reduce the enormous peak powers in amplifiers, which would otherwise limit the output energy due to optical damage of the amplifier components and intensity-dependent pulse distortions like self focusing and self phase modulation as mentioned before in chapter 4 .

Typically pulses are temporally stretched with a dispersive delay line introducing different velocities or path lengths to different frequency components of the pulse leading to much longer pulse duration with reduced peak intensity. Following the amplification, pulses are recompressed by another delay line introducing exactly the opposite dispersion of the initial stretcher. Dispersion can be introduced by dispersive bulk material or grating based delay lines.

\subsection{Stretcher and compressor design}

As the refractive index in an optical medium is frequency dependent a pulse broadens while traveling through dispersive material and is therefore temporally stretched. A pulse with the initial duration $T_{0}$ (where $T_{0}$ is the temporal width of a Gaussian pulse given at the $1 / \mathrm{e}$ intensity point) traveling the distance $\mathrm{z}$ through a dispersive material with the group velocity dispersion (GVD) $\beta_{2}$ is according to [94] stretched to the duration

$$
\mathrm{T}_{1}=\mathrm{T}_{0} \sqrt{1+\left(\frac{\mathrm{z}\left|\beta_{2}\right|}{\mathrm{T}_{0}^{2}}\right)^{2}}
$$

whereas the group delay (GD) is defined as the derivative of the change in spectral phase $\Psi$ in radiant with respect to the angular frequency $\Omega$ in radiant/s at the central signal frequency $\omega_{\mathrm{s}}$

$$
\mathrm{GD}=\left.\frac{\mathrm{d} \Psi}{\mathrm{d} \Omega}\right|_{\omega_{\mathrm{s}}}
$$

and the group velocity dispersion GVD is defined as the frequency dependency of the group delay, or (quantitatively) the corresponding derivative with respect to angular frequency

$$
\mathrm{GVD}=\left.\frac{\mathrm{d}^{2} \Psi}{\mathrm{d} \Omega^{2}}\right|_{\omega_{\mathrm{s}}}
$$

With the conversion [94] of the 1/e-intensity pulse duration $T_{0}$ into the full width half maximum pulse duration

$$
\mathrm{T}_{\mathrm{FWHM}}=2 \sqrt{\ln 2} \mathrm{~T}_{0},
$$

the required group delay dispersion (GDD) to stretch the pulse of the duration $\mathrm{T}_{\text {in }}$ to the duration $\mathrm{T}_{\text {out }}$ can be calculated with equation 6-1 to

$$
\text { GDD }=z\left|\beta_{2}\right|=\frac{T_{\text {in }}}{4 \ln 2} \sqrt{T_{\text {out }}^{2}-T_{\text {in }}^{2}}
$$


The calculation of the required GDD to stretch input pulses $T_{\text {in }}$ from $1.0 \mathrm{ps}, 1.5 \mathrm{ps}$ and $2.0 \mathrm{ps}$ respectively to different pulse durations $\mathrm{T}_{\text {out }}$ is displayed in figure 6-1. The calculated output pulse duration versus the input pulse duration for dispersive pulse broadening with different levels of GDD is shown in figure 6-2. Shorter pulses are increasingly sensitive to dispersion. Substantial broadening occurs when the square of the pulse duration is smaller than the group delay dispersion.

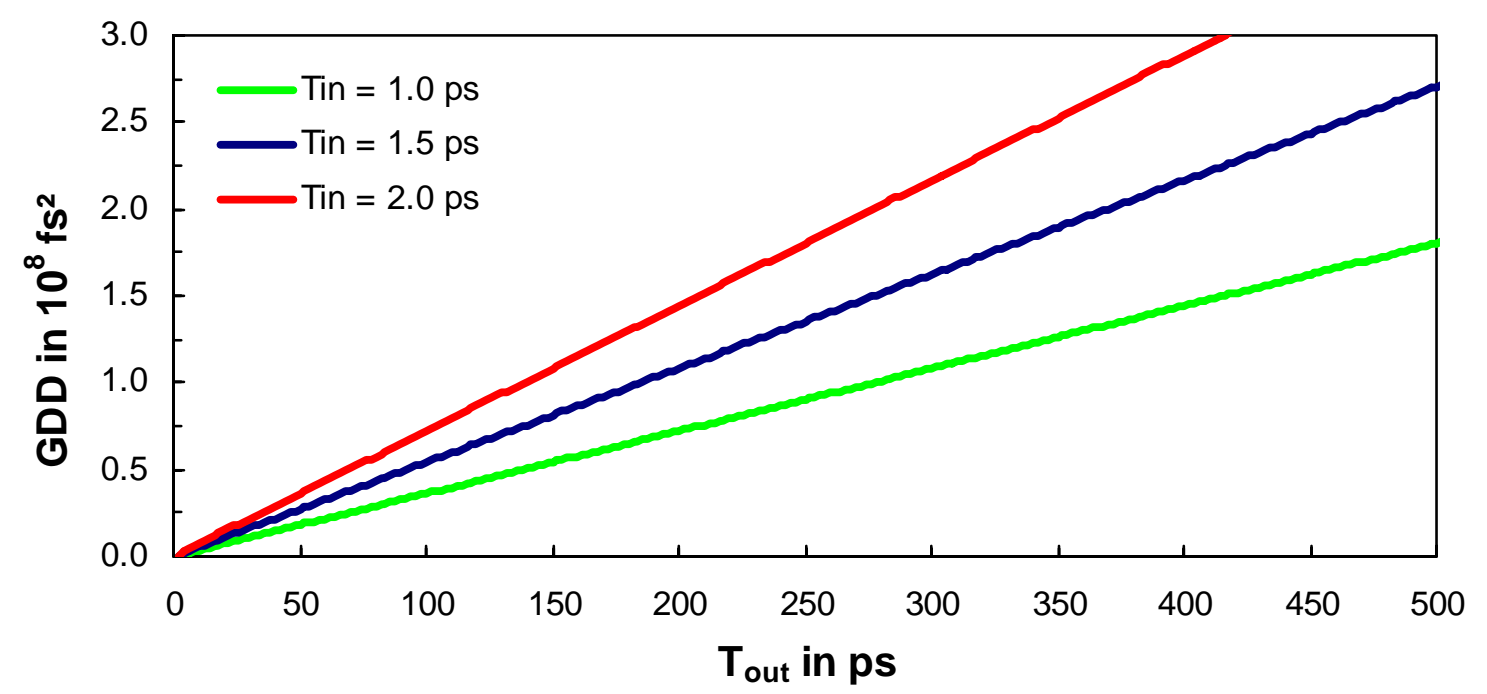

Fig. 6-1 Required group delay dispersion to stretch a pulse to the time duration $T_{\text {out }}$ calculated for three different time-bandwidth limited input pulse durations.

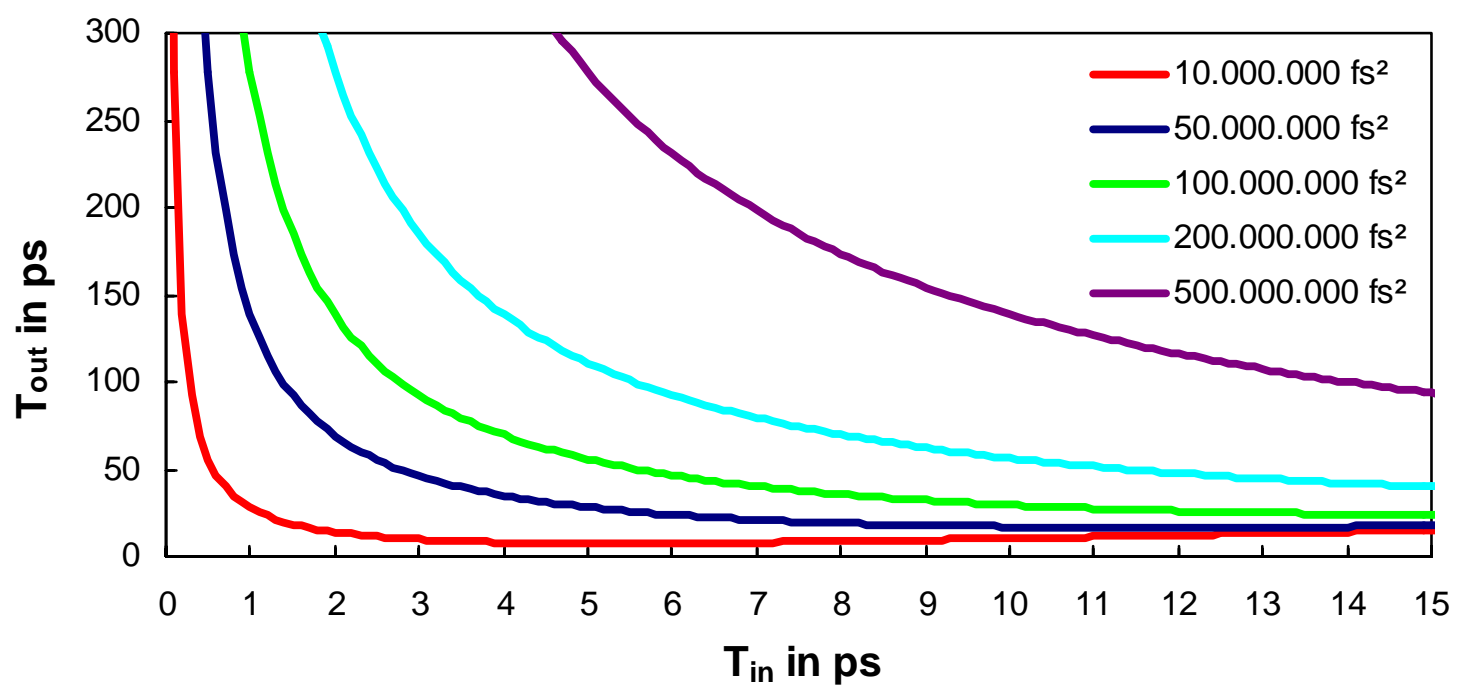

Fig. 6-2 Output pulse duration versus time-bandwidth limited initial pulse durations for dispersive pulse broadening with different levels of group delay dispersion (GDD). Shorter pulses are increasingly sensitive to dispersion. Substantial broadening occurs when the square of the pulse duration is smaller than the group delay dispersion.

For stretching the pulses before the amplification of the regenerative amplifier two different delay lines were used introducing a GDD of $3.18 \cdot 10^{7} \mathrm{fs}^{2}$ and $1.185 \cdot 10^{8}$ fs respectively. Figure 6-3 shows the output pulse duration versus the initial pulse duration for dispersive pulse broadening with the two levels of group delay dispersion $3.18 \cdot 10^{7} \mathrm{fs}^{2}$ and $1.185 \cdot 10^{8} \mathrm{fs}^{2}$ realized to stretch the seed pulses of the following described laser systems. 


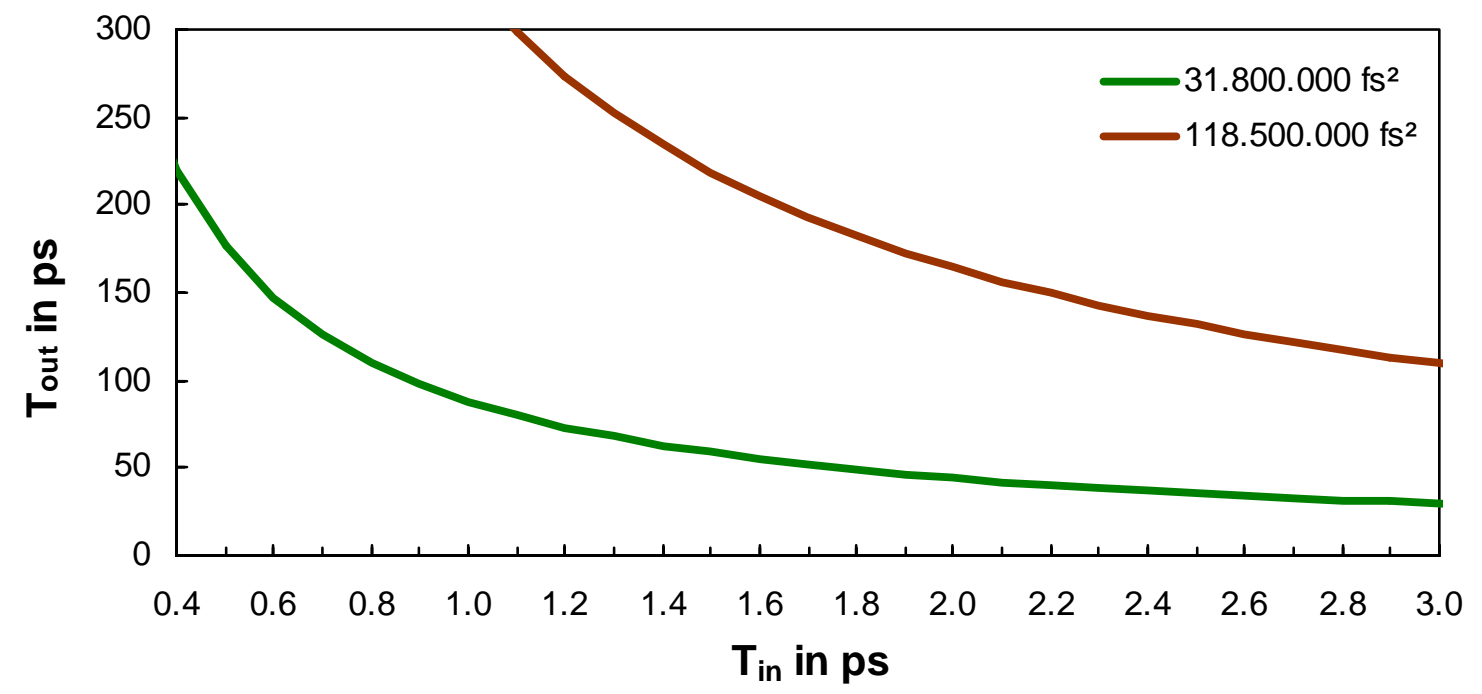

Fig. 6-3 Output pulse duration versus the time bandwidth limited initial pulse duration for dispersive pulse broadening with the two levels of group delay dispersion (GDD) $3.18 \cdot 10^{7} \mathrm{fs}^{2}$ and $1.185 \cdot 10^{8}$ fs used in this work for stretching the seed pulses.

Bulk materials like glass have rather low group velocity dispersions and to stretch a time bandwidth limited pulse at a center wavelength of $1030 \mathrm{~nm}$ from $2 \mathrm{ps}$ to $200 \mathrm{ps}$ more than $12 \mathrm{~km}$ of fused silica would be required. To introduce a large amount of GDD to a pulse, grating based delay lines can be chosen. Fortunately, at wavelengths around $1 \mu \mathrm{m}$ stretchers and compressors of insignificant loss are available utilizing transmission or reflection gratings with efficiencies as high as $97 \%$ and $99 \%$ respectively [30,31].

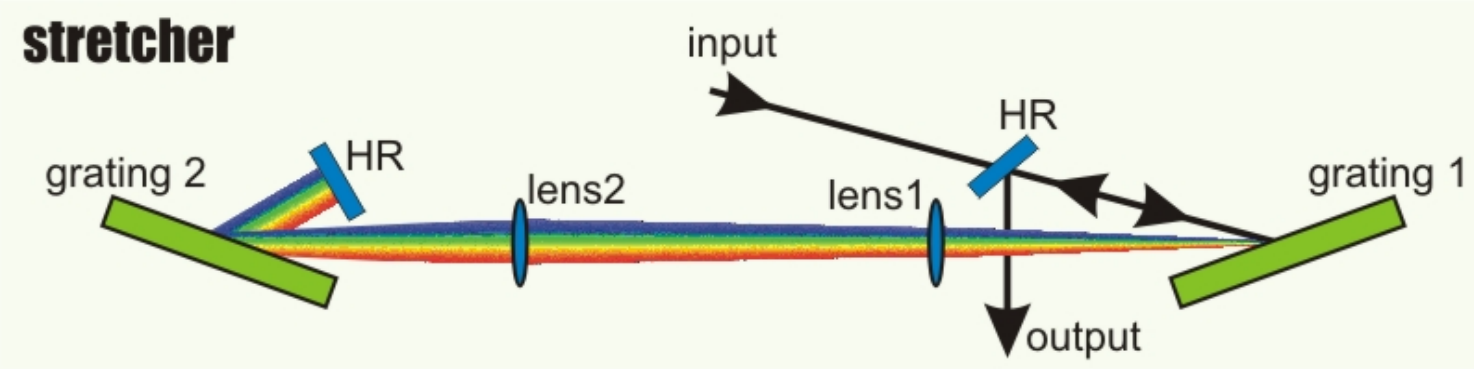

Fig. 6-4 Schematical pulse stretcher assembly introducing positive group velocity dispersion.

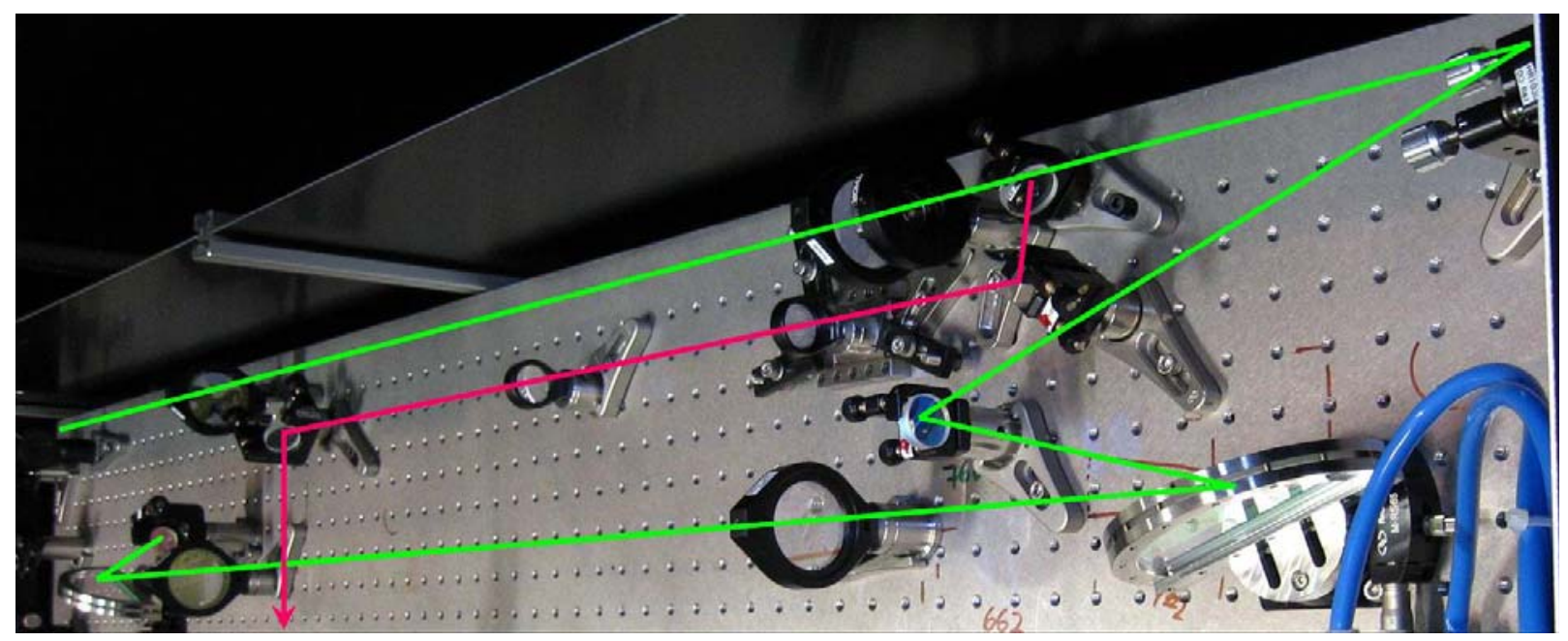

Fig. 6-5 Picture of the 4f-stretcher setup introducing positive group velocity dispersion. 
Before amplification in the here presented laser system, the seed pulses are temporally dispersed in a 4f-stretcher consisting of a pair of reflection gratings with 1740 lines $/ \mathrm{mm}$, inserted at an angle of incidence of $59^{\circ}$ and two lenses $(\mathrm{f}=1000 \mathrm{~mm})$ similar to the delay line presented in [103] and schematically shown in figure 6-4. A picture of the stretcher is shown in figure 6-5. For a grating, the angle of incidence $\beta$ and the diffraction angle $\beta^{\prime}$ are related via the grating equation

$$
\sin \beta^{\prime}-\sin \beta=\frac{2 \pi \mathrm{c}}{\Omega \mathrm{d}}
$$

with the grating constant $d$, the angular frequency $\Omega$ in radiant/s and the speed of light $\mathrm{c}$. Using the grating equation the angular dispersion of a grating is obtained as

$$
\left.\frac{\mathrm{d} \beta^{\prime}}{\mathrm{d} \Omega}\right|_{\omega_{\mathrm{s}}}=-\frac{2 \pi \mathrm{c}}{\omega_{\mathrm{s}}^{2} \mathrm{~d} \cos \beta^{\prime}}
$$

and leads in a compressor setup shown in figure 6-4 with the length L between the two gratings and the focal lengths $f_{1}$ and $f_{2}$ of the two lenses to the overall dispersion

$$
\left.\frac{\mathrm{d}^{2} \Psi}{\mathrm{d} \Omega^{2}}\right|_{\omega_{\mathrm{s}}}=-\frac{\omega_{\mathrm{s}}}{\mathrm{c}}\left(\left.\frac{\mathrm{d} \beta^{\prime}}{\mathrm{d} \Omega}\right|_{\omega_{\mathrm{s}}}\right)^{2}\left[\mathrm{~L}-2\left(\mathrm{f}_{1}+\mathrm{f}_{2}\right)\right]\left(\frac{\mathrm{f}_{1}}{\mathrm{f}_{2}}\right)^{2}
$$

In a double pass configuration the two realized stretcher set ups introduced a GDD of $3.18 \cdot 10^{7} \mathrm{fs}^{2}$ in a short stretcher, $1.19 \cdot 10^{8} \mathrm{fs}^{2}$ in a long setup at $70 \%$ optical throughput. For the short stretcher, two lenses with the focal length $\mathrm{f}=380 \mathrm{~mm}$ were used in a $1276 \mathrm{~mm}$ long setup, reaching the desired GDD of $3.18 \cdot 10^{7} \mathrm{fs}^{2}$. The two lenses were separated by the distance of $760 \mathrm{~mm}$ and $255 \mathrm{~mm}$ separated from the gratings respectively. The second, long stretcher contained two lenses with the focal length $\mathrm{f}=1000 \mathrm{~mm}$ separated by $2 \mathrm{~m}$ centered in the $\mathrm{L}=2727 \mathrm{~mm}$ long folded setup resulting in the overall GDD of $1.19 \cdot 10^{8} \mathrm{fs}^{2}$. Following the amplification a negative GDD needs to be introduced by a compressor to compensate for the positive GDD of the stretcher. In this work recompression is performed using two transmission gratings with 1400 lines/mm arranged in a double pass Littrow-configuration as shown in figure 6-6 with overall transmission of $\sim 77 \%$ [30].

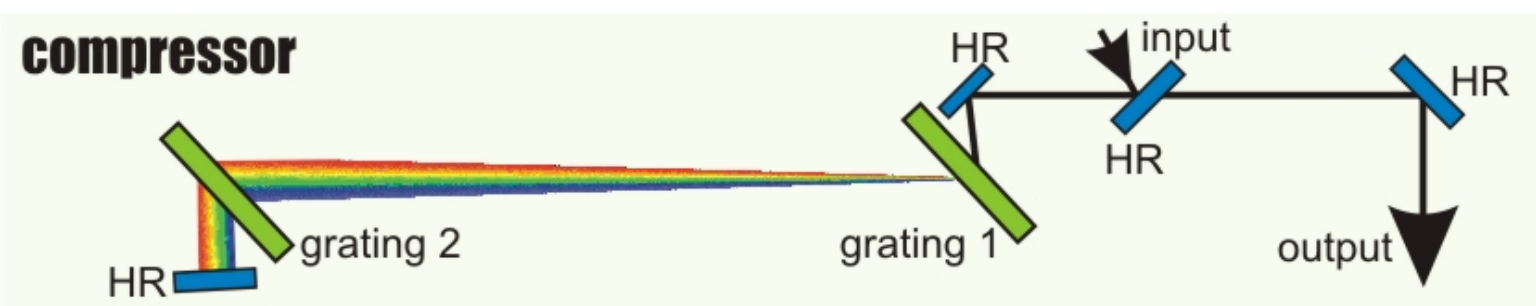

Fig. 6-6 Schematical pulse compressor assembly introducing negative group velocity dispersion.

With $b$ being the normal separation of the gratings, the overall GDD of the compressor is given by

$$
\left.\frac{\mathrm{d}^{2} \Psi}{\mathrm{d} \Omega^{2}}\right|_{\omega_{\mathrm{s}}}=-\frac{\lambda_{\mathrm{s}}}{2 \pi \mathrm{c}^{2}}\left(\frac{\lambda_{\mathrm{s}}}{\mathrm{d}}\right)^{2} \frac{\mathrm{b}}{\cos ^{3} \beta^{\prime}\left(\lambda_{\mathrm{s}}\right)}
$$


In terms of the distance

$$
\mathrm{L}=\frac{\mathrm{b}}{\cos \beta^{\prime}}
$$

between the two gratings along the ray with the signal frequency $\omega_{1}$ at $\Omega=\omega_{1}$, equation 6-9 can be written

$$
\left.\frac{\mathrm{d}^{2} \Psi}{\mathrm{d} \Omega^{2}}\right|_{\omega_{\mathrm{s}}}=-\frac{\lambda_{\mathrm{s}}}{2 \pi \mathrm{c}^{2}}\left(\frac{\lambda_{\mathrm{s}}}{\mathrm{d}}\right)^{2} \frac{\mathrm{L}}{\cos ^{2} \beta^{\prime}\left(\lambda_{\mathrm{s}}\right)}
$$

where

$$
\cos ^{2} \beta_{\mathrm{s}}\left(\omega_{1}\right)=1-\left(\frac{2 \pi \mathrm{c}}{\omega_{1}}+\sin \beta\right) .
$$

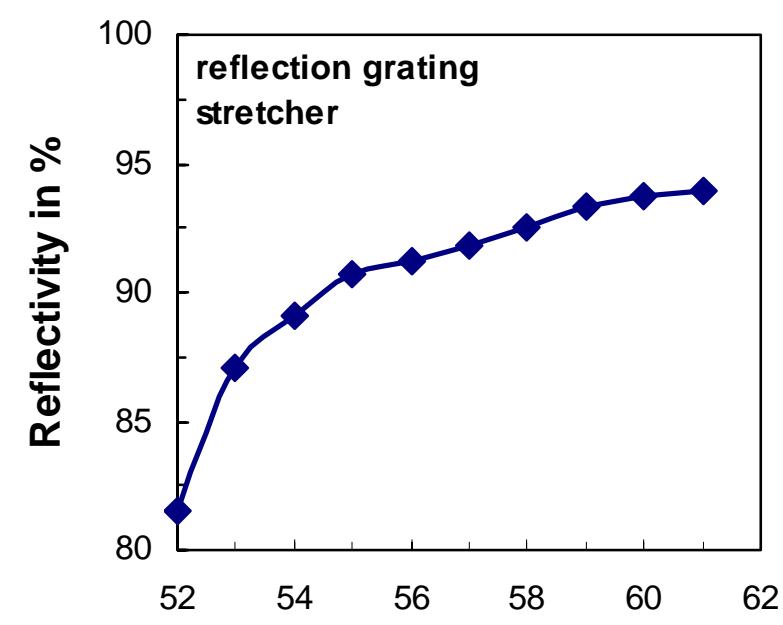

Angle of incidence in degree

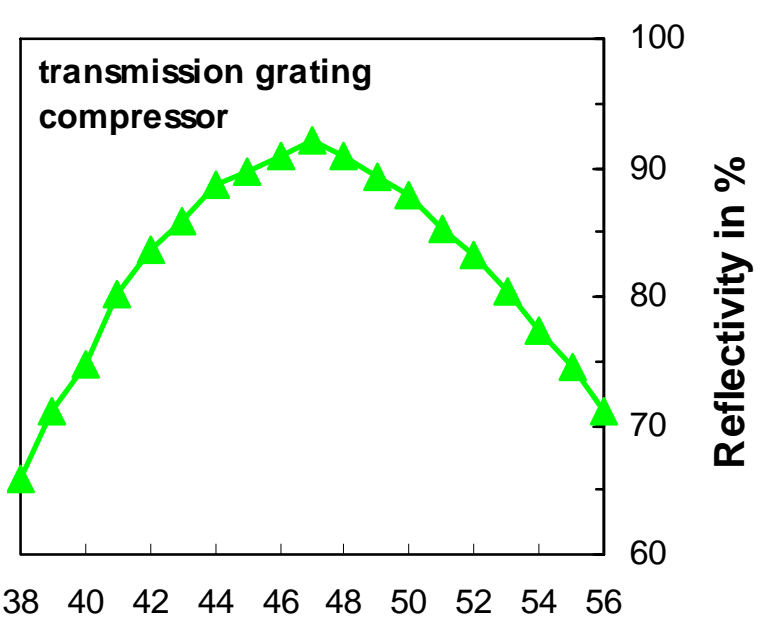

Angle of incidence in degree

Fig. 6-7 Measured reflectivities of the reflection gratings used in the stretcher (left side) and of the transmission gratings installed in the compressor (right side).
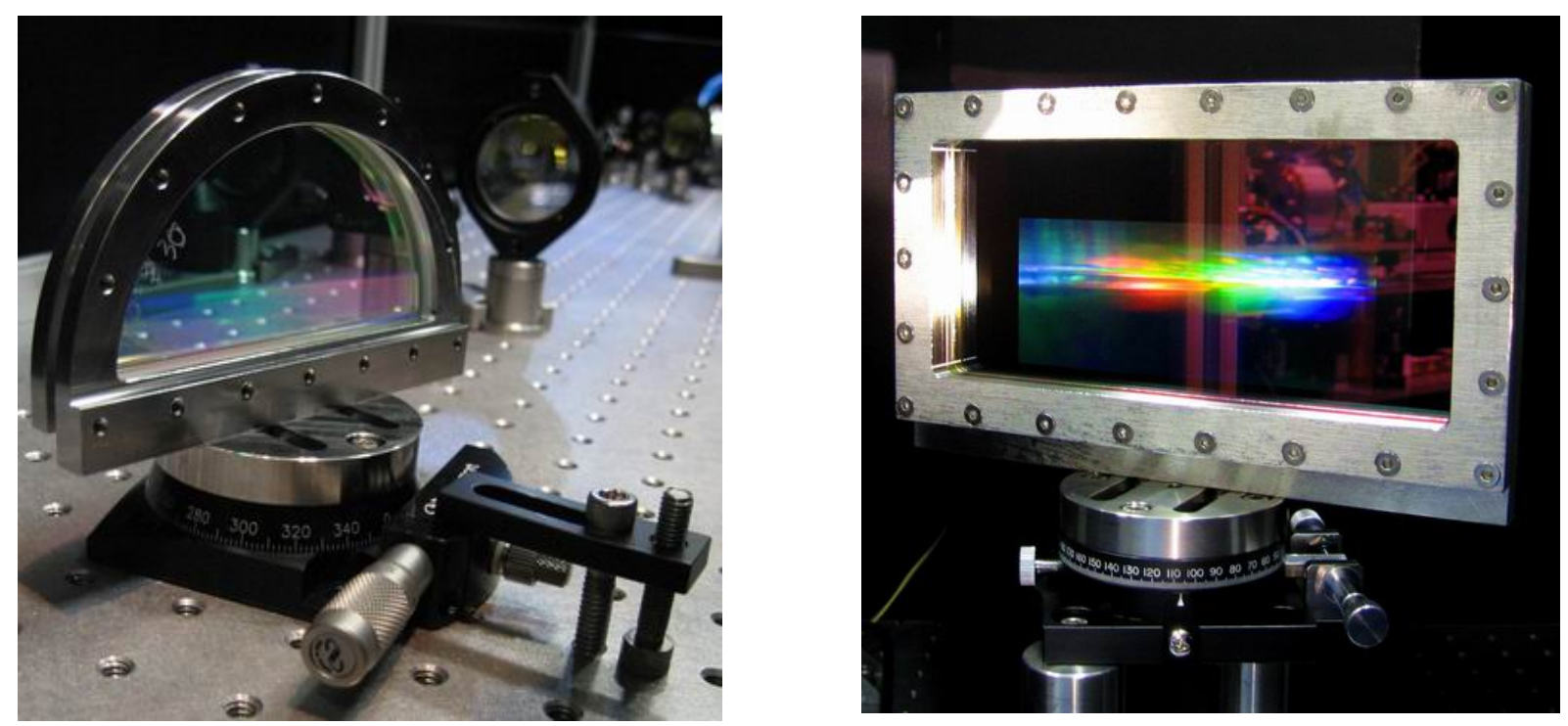

Fig. 6-8 Reflection grating used in the compressor with 1740 lines $/ \mathrm{mm}$ produced at Lawrence Livermore National Laboratory (LLNL) cut into half from a $100 \mathrm{~mm}$ diameter sample (left side) and the transmission grating with $\mathbf{1 4 0 0}$ lines/mm produced at Friedrich Schiller University Jena with a size of $50 \mathrm{~mm} \times 120 \mathrm{~mm}$ (right side). 
In a double pass configuration, the two built compressor introduced a GDD of $-3.18 \cdot 107 \mathrm{fs}^{2}$ in a short setup of the normal separation $b=1.46 \mathrm{~m}$ and a GDD of $-1.19 \cdot 108 \mathrm{fs}^{2}$ in a long compressor of the normal separation $b=5.47 \mathrm{~m}$.

Figure 6-7 shows the measured reflectivities of the reflection gratings used in the stretcher (left side) and of the transmission gratings installed in the compressor (right side). Figure 6-8 displays the reflection grating used in the compressor with 1740 lines/mm produced at Lawrence Livermore National Laboratory (LLNL) cut into half from a $100 \mathrm{~mm}$ diameter sample (left side) and the trans-mission grating with 1400 lines/mm produced at Friedrich Schiller University Jena with a size of $50 \mathrm{~mm} \times 120 \mathrm{~mm}$ (right side).

\subsection{Results from the IFSW disk based regenerative amplifier with stretched seed pulses}

Applying a GDD of $3.18 \cdot 10^{7} \mathrm{fs}^{2}$ to the $530 \mathrm{fs}$ long seed pulses of the $\mathrm{Yb}: \mathrm{KGW}$ by the short stretcher described in chapter 6.1 resulted in chirped seed pulse durations around $120 \mathrm{ps}$ at a pump current of $18 \mathrm{~A}$. The measured autocorrelation traces of the stretched seed pulses provided by the $\mathrm{Yb}: \mathrm{KGW}$ oscillator for various pump currents are shown in figure 6-9.

Figure 6-10 illustrates the absence of spectral broadening due to SPM in the regenerative amplifier after amplifying the stretched seed pulses to a few millijoule pulse energy. The spectrum is shifted after 100 round trips in the regenerative amplifier resonator to the $\mathrm{Yb}: \mathrm{YAG}$ gain maximum at a wavelength of $1030 \mathrm{~nm}$.

The pulses could be amplified up to a maximum pulse energy of $4.0 \mathrm{~mJ}$ at a repetition rate of $10 \mathrm{kHz}$. At higher pulse energies the disk was damaged and no further measurements or improvements of the cavity design to achieve even higher output energies could be taken.

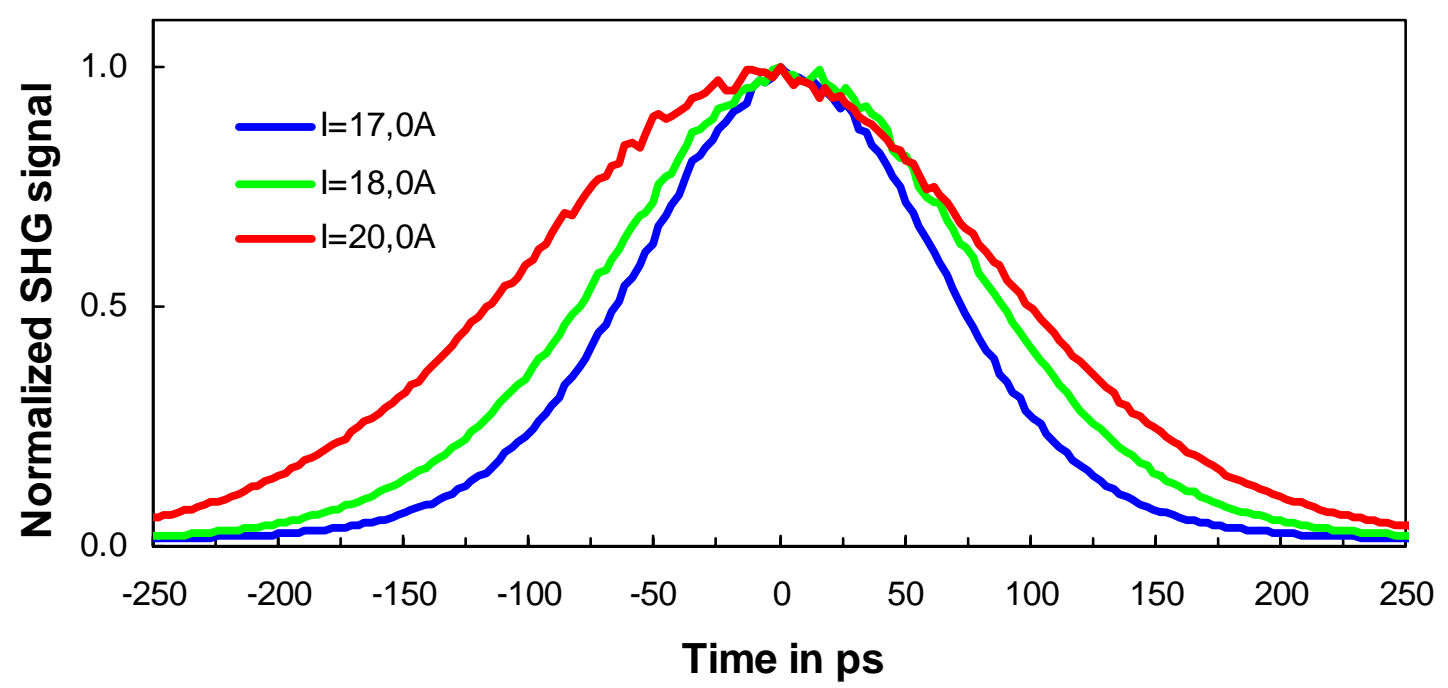

Fig. 6-9 Autocorrelation trace of the stretched seed pulses provided by the Yb:KGW oscillator for various pump currents.

The comparison of the amplified chirped pulse duration and the stretched $\mathrm{Yb}: \mathrm{KGW}$ oscillator pulse duration is shown in figure 6-11. During amplification substantial gain narrowing is 
observed, resulting in a shortening of the chirped pulse duration inside the regenerative amplifier cavity to approximately $70 \mathrm{ps}$.

The optical quality of the used Yb:YAG disk was rather low and most disks had damage spots on the surface or at the high reflecting coating on the back side from the beginning. To align the seed laser through the amplifier cavity, a camera was used to find the position of the pump light spot on the Yb:YAG disk. Therefore, a few Watts of pump light were applied to the disk and the position of the pump spot was determined with the camera and later carefully overlapped with the seed laser beam.

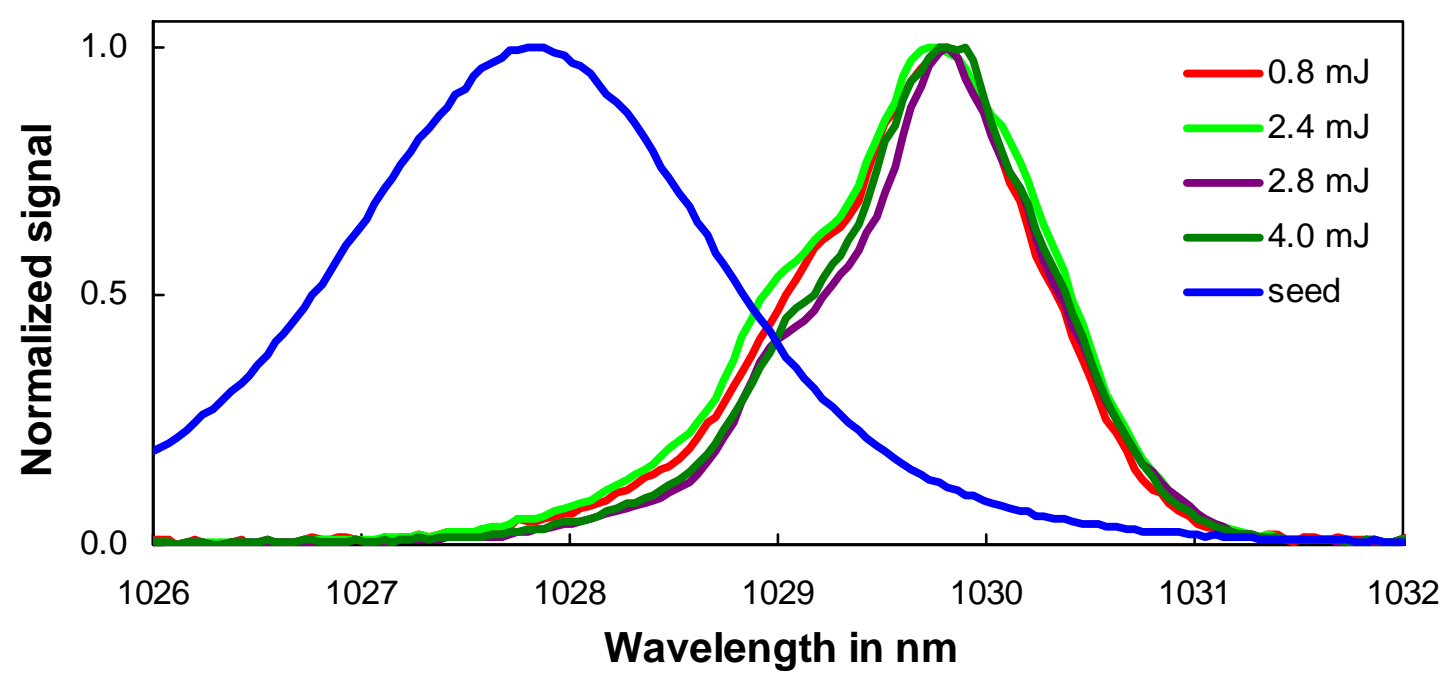

Fig. 6-10 Spectra of the stretched pulses amplified pulses to various energies at a repetition rate of $10 \mathrm{kHz}$ compared to the $\mathrm{Yb}: \mathrm{KGW}$ seed laser spectra.

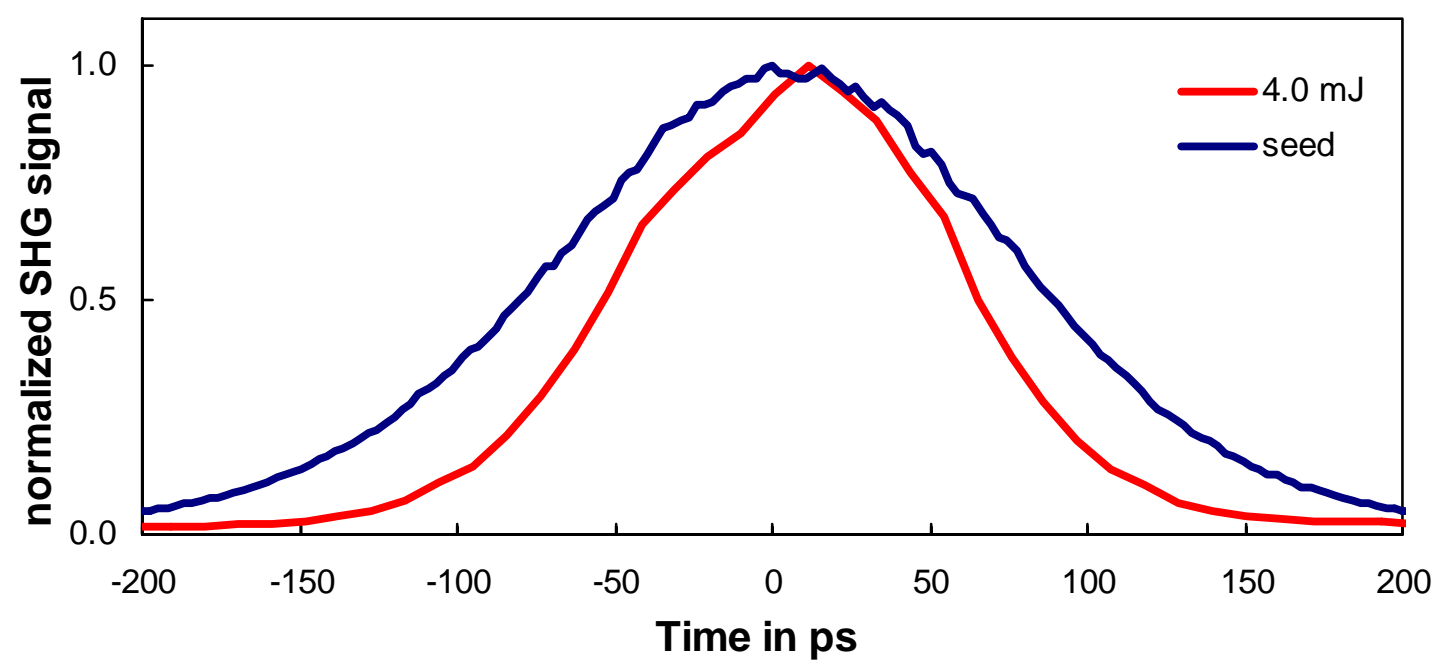

Fig. 6-11 Autocorrelation trace of the amplified $4 \mathrm{~mJ}$ pulse at a repetition rate of $10 \mathrm{kHz}$ in comparison to the stretched Yb:KGW seed laser pulse. The effect of gain narrowing leading to shorter amplified pulses with respect to the seed pulse duration is clearly visible.

The same camera was used to check the quality of the disks and small scattering spots could be observed even on new disks as it is shown in figure 6-12. The round pump spot occurs oval on the picture due to the inclined mounted camera, observing the disk from the top of the amplifier module as shown in figure 5-1. 
Mounted inside the amplifier module, the disk was mostly damaged in operation at energy levels of the amplified pulse between 2.0 and $4.0 \mathrm{~mJ}$ always at defects already existent on the new disks. Figure 6-13 shows the new and the damaged disk number L799 in comparison. Clearly, it can be seen that the optical damage in operation arose at the same position where already a defect on the new disk could be observed.

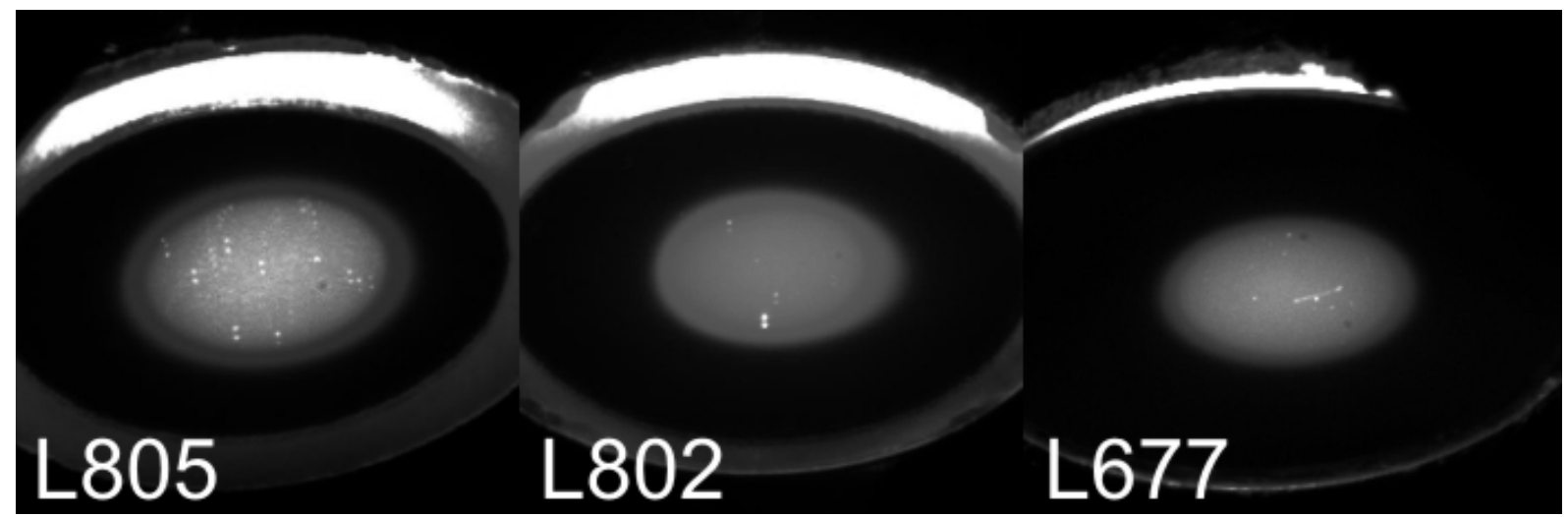

Fig. 6-12 Pictures of the implemented Yb:YAG disks L805, L802 \& L677 inside the amplifier head taken with the camera which was used for aligning the seed laser inside the regenerative amplifier through the pumped area.

Not only bad optical quality made it difficult to operate the system at the desired pulse energy above $5.0 \mathrm{~mJ}$ but low availability combined with long waiting times of several weeks for replacement disks made it impossible to work continuously. Caused by the manual soldering process of the disk onto the cooling plate each manufactured replacement disk was furnished with a different radius of curvature demanding new cavity designs each time a disk was replaced. In consequence of the above mentioned problems the amplifier was replaced by a disk laser head from Trumpf Laser GmbH + Co. KG.
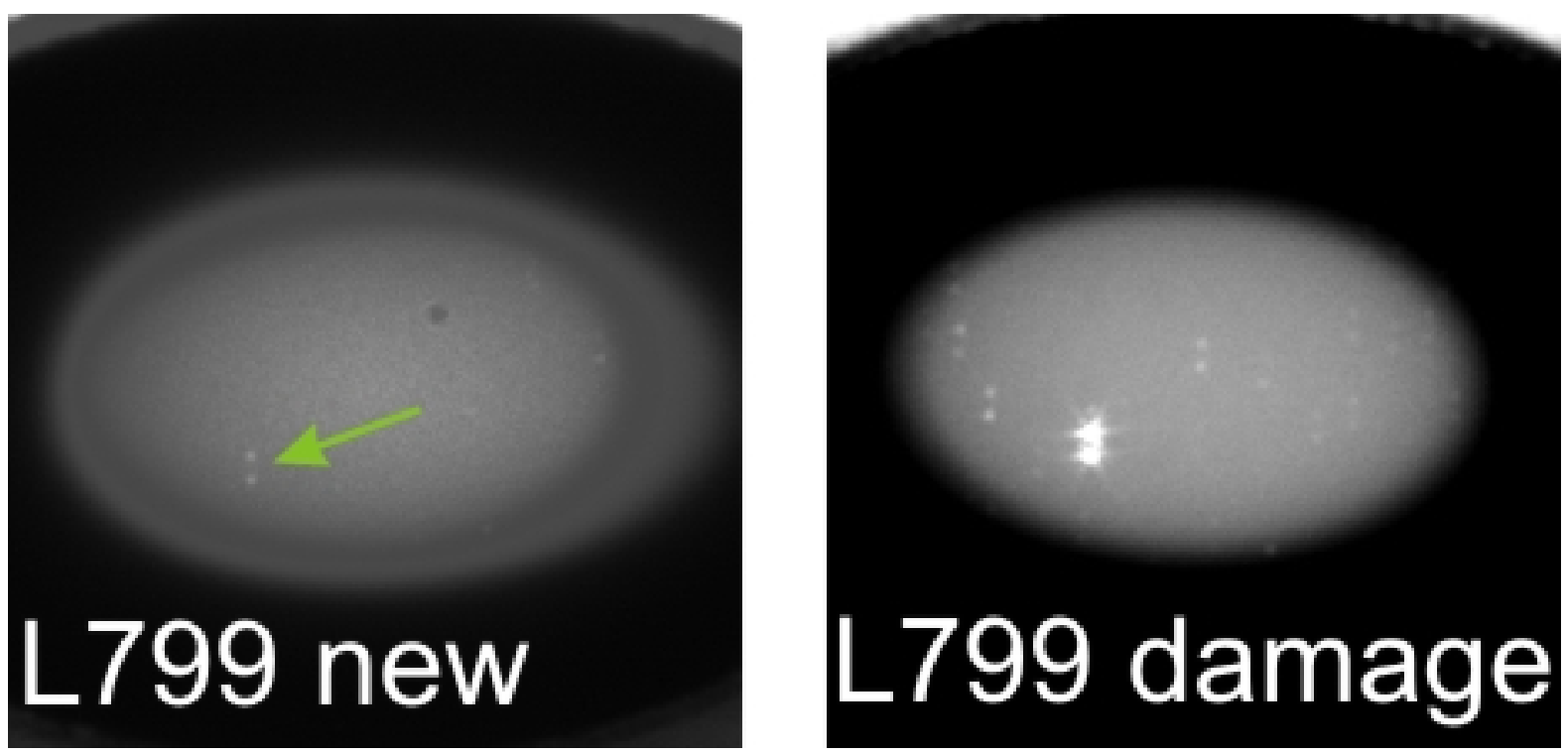

Fig. 6-13 The new disk no. L799 mounted in the amplifier head (left side) and with optical damage after amplifying the pulses above $3 \mathrm{~mJ}$ (right side). 


\subsection{Results from the Trumpf Laser GmbH disk based regenera- tive chirped pulse amplifier}

The following experiments were realized utilizing a disk laser amplifier head identical to the $5 \mathrm{~kW}$ laser head implemented in the commercial products of Trumpf Laser + Co. KG already presented in figure 2-2. Figure 6-14 demonstrates the damage-free pump spot of the disk compared to mm-groves of a ruler together with the pumped disk inside the amplifier head.

High optical quality of the Yb:YAG disks provided by Trumpf Laser allowed to reduce the spot size of the laser beam on the disk to $2.4 \mathrm{~mm}$ resulting in a pump spot diameter of $3.0 \mathrm{~mm}$. Despite the higher pump density the $\sim 1 / 10 \mathrm{~mm}$ thin Yb:YAG disk mounted on a diamond heat sink is efficiently cooled and develops only a weak thermal lens over a broad range of pump power as shown in figure 6-15 compared to the earlier used disk mounted in the IFSW laser head. For the first experiments, a cheaper plane-parallel disk was used inside the amplifier head and after the optimization of the system, it was exchanged to a 0.1 degree wedged disk. The experimental results are described in the following chapters 6.3.1 and 6.3.3.
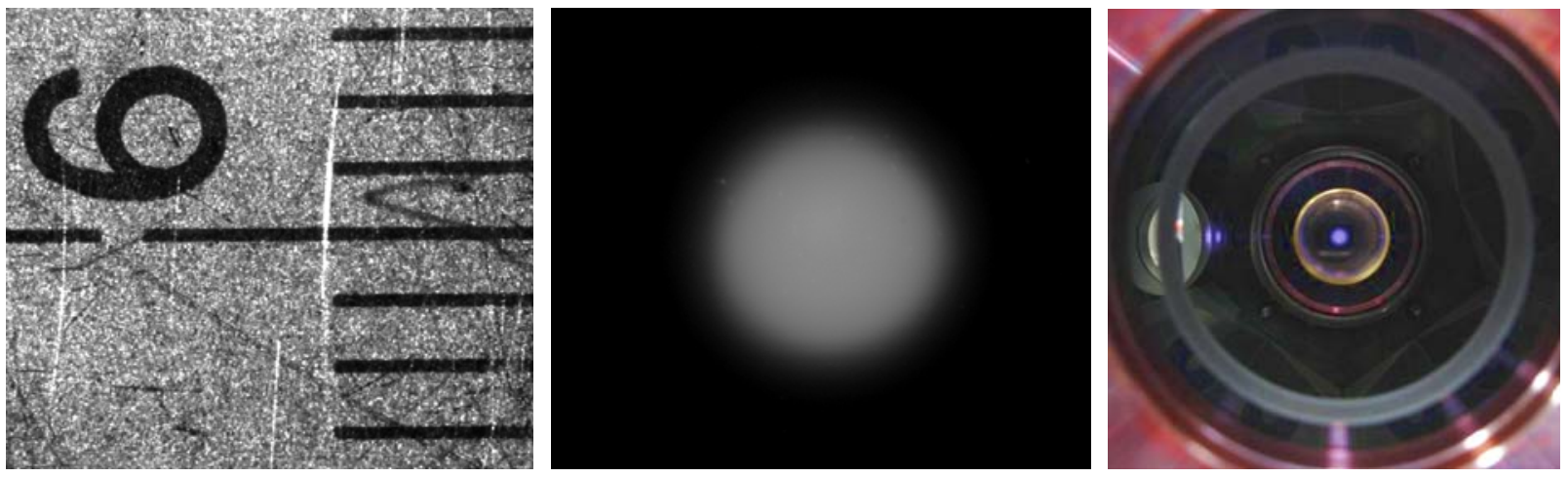

Fig. 6-14 Damage-free pump spot (center) compared to the mm-groves of a ruler (left side) and the pumped disk inside the laser amplifier.

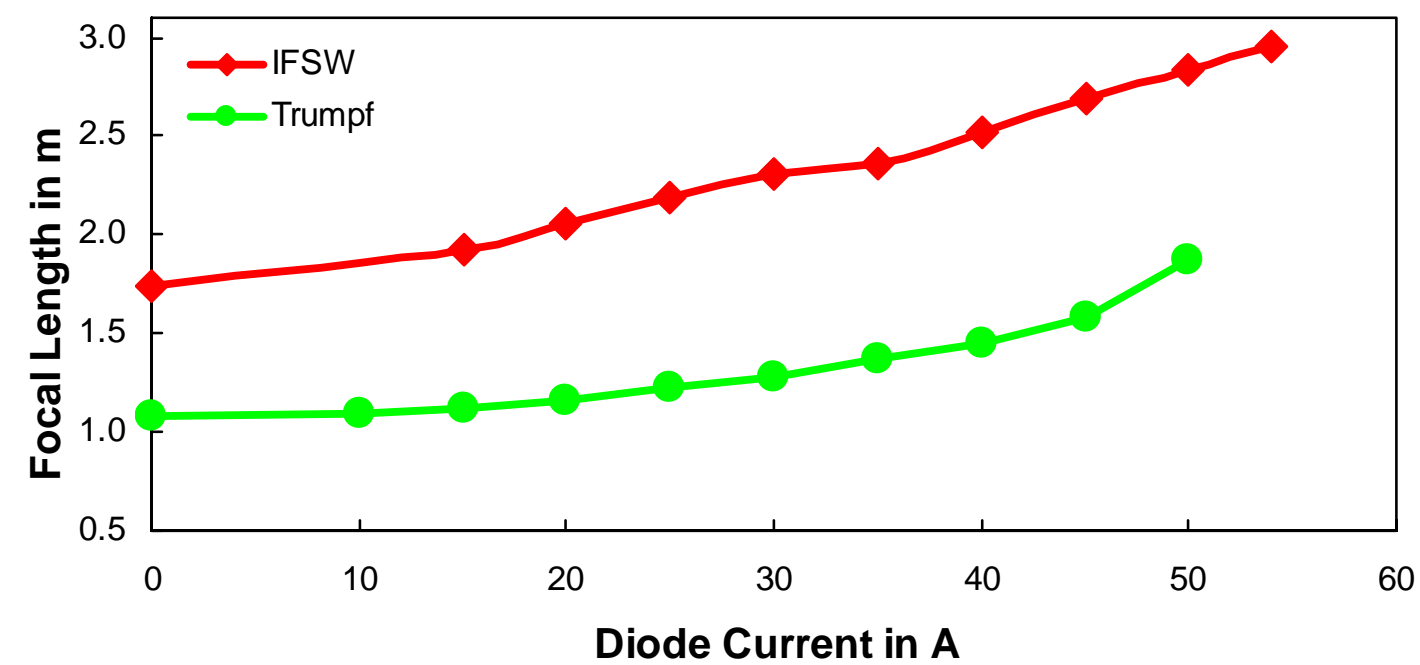

Fig. 6-15 Change of the thermal induced lens of the Yb:YAG disks in a Trumpf and a IFSW laser head versus the absorbed pump radiation $(10 \mathrm{~A} \sim 100 \mathrm{~W})$. 
Higher pump power density results in a higher gain per single pass via the disk and allows a simplified cavity design with only one double pass through the gain medium per round trip in the amplifier resonator. Together with a smaller beam diameter of approximately $3.0 \mathrm{~mm}$ in the PC enabled by the CPA setup, a simple cavity design could be used leading to stable and reliable day to day performance of the system. The cavity design is shown in figure 6-16. The exact used components and distances are listed in table 6-1.

Together with the amplifier head, also the seed source of the system was exchanged to the ultra-broadband Ti:sapphire oscillator described in chapter 3.2 to achieve the designated optical synchronization.

The complete apparatus consists of a Ti:sapphire based seed laser, a stretcher, a pulse picker, a mode matching telescope, an amplifier resonator and a compressor. The setup is shown in a simplified way and schematically in figure 6-17.

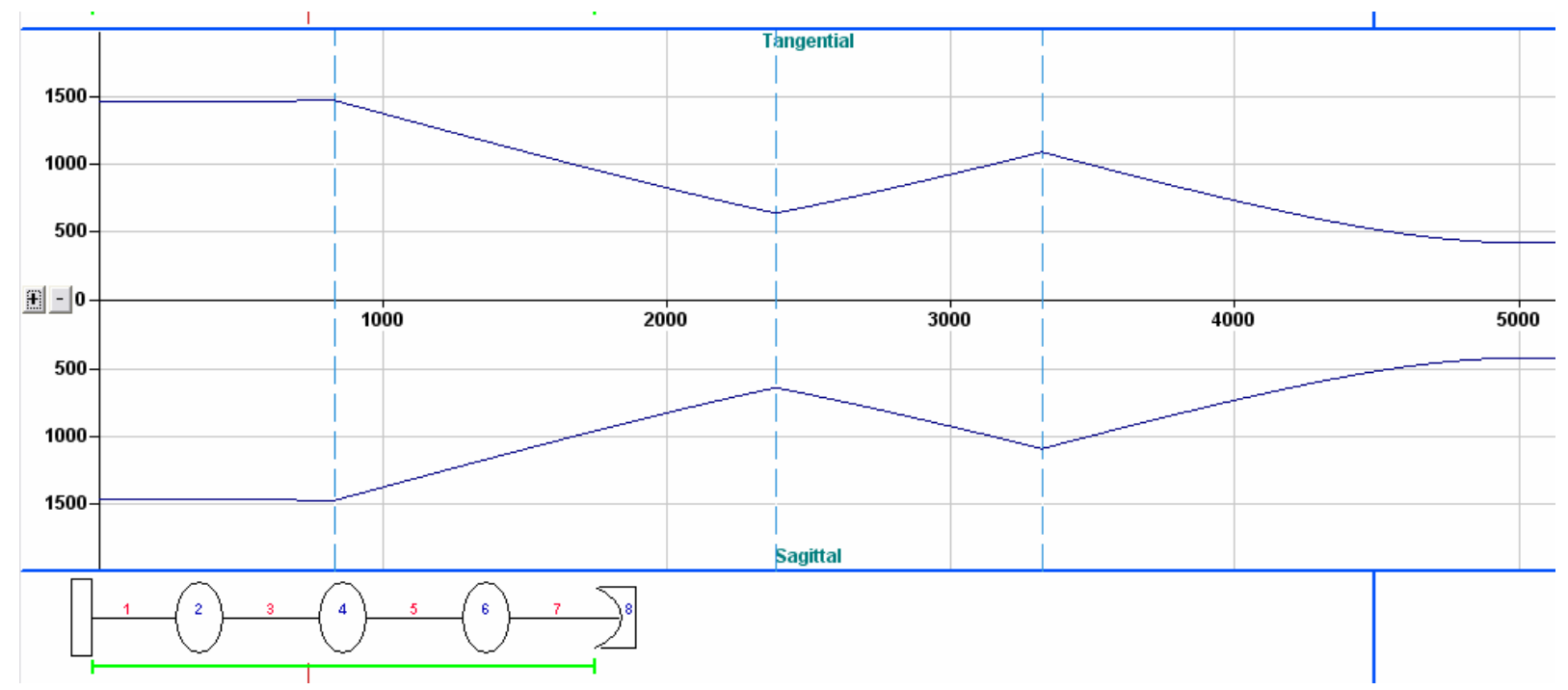

Fig. 6-16 Cavity design of the Yb:YAG disk based regenerative amplifier with one pass through the disk.

\begin{tabular}{c|c}
\hline mirror / distance / disk & radius of curvature / distance in $\mathrm{mm}$ \\
\hline end mirror & flat \\
distance & 825 \\
mirror concave & -5000 \\
distance & 1550 \\
mirror convex & +750 \\
distance & 935 \\
disk concave & -1020 \\
distance & 1860 \\
end mirror concave & -3000 \\
\hline
\end{tabular}

Tab. 6-1 Distances and used mirrors in the designed cavity displayed in figure 6-15 listed from the left to the right side.

In a double pass configuration, the stretcher introduces a group delay dispersion of $3.18 \cdot 10^{7} \mathrm{fs}^{2}$ and $75 \%$ throughput. Due to the finite size of the used optical components, the 
implemented stretcher supports a bandwidth of $\sim 10 \mathrm{~nm}$ which is still well above the estimated $\sim 5.3 \mathrm{~nm}$ gain bandwidth of Yb:YAG and cuts off all other wavelength. Taking only the supported gain bandwidth of $\mathrm{Yb}: Y A G$ into account, the stretched pulse duration is estimated to be $\sim 300$ ps.

The expected bandwidth reduced by gain narrowing of the amplified pulse is estimated to be $\sim 1 \mathrm{~nm}$ leading to a calculated stretched pulse duration inside the amplifier of $56.5 \mathrm{ps}$. Figure 6-18 shows the seed spectra provided by the Ti:sapphire oscillator together with the seed beam profile after the stretcher. The calculated Fourier-transform limited pulse duration of $230 \mathrm{fs}$ considering the spectra supported by the stretcher is shown in Figure 6-19. The average seed power after the stretcher was measured to be between 80 and $120 \mu \mathrm{W}$ depending on the daily performance and alignment of the Ti:sapphire oscillator and results in a total seed energy between 1.14 and $1.71 \mathrm{pJ}$.

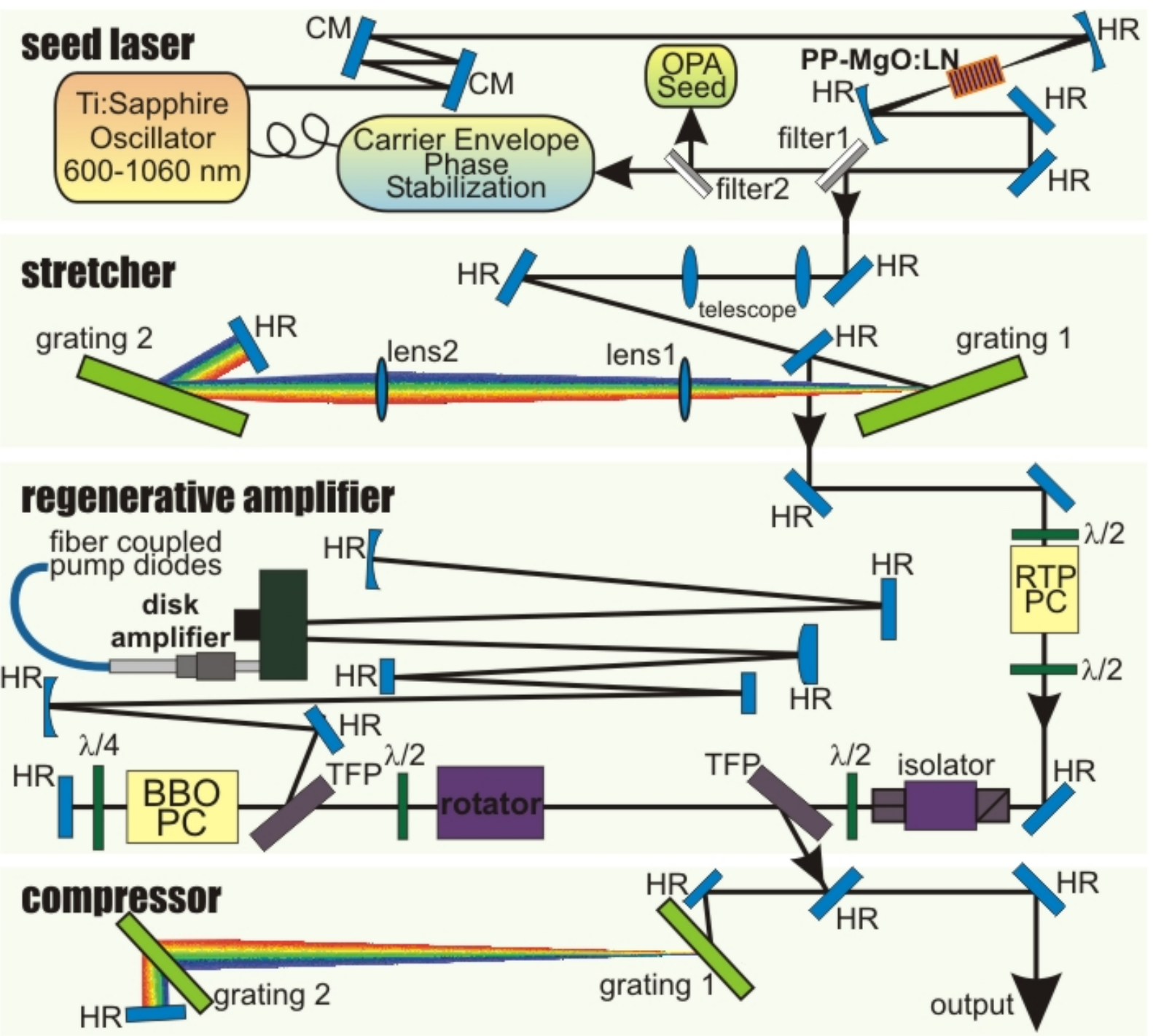

Fig. 6-17 Schematical setup of the regenerative amplifier. HR, high reflecting mirror; CM, chirped mirror; PC, Pockels cell; TFP, thin-film polarizer; $\lambda / 2$, half-wave plate; $\lambda / 4$, quarter-wave plate. 


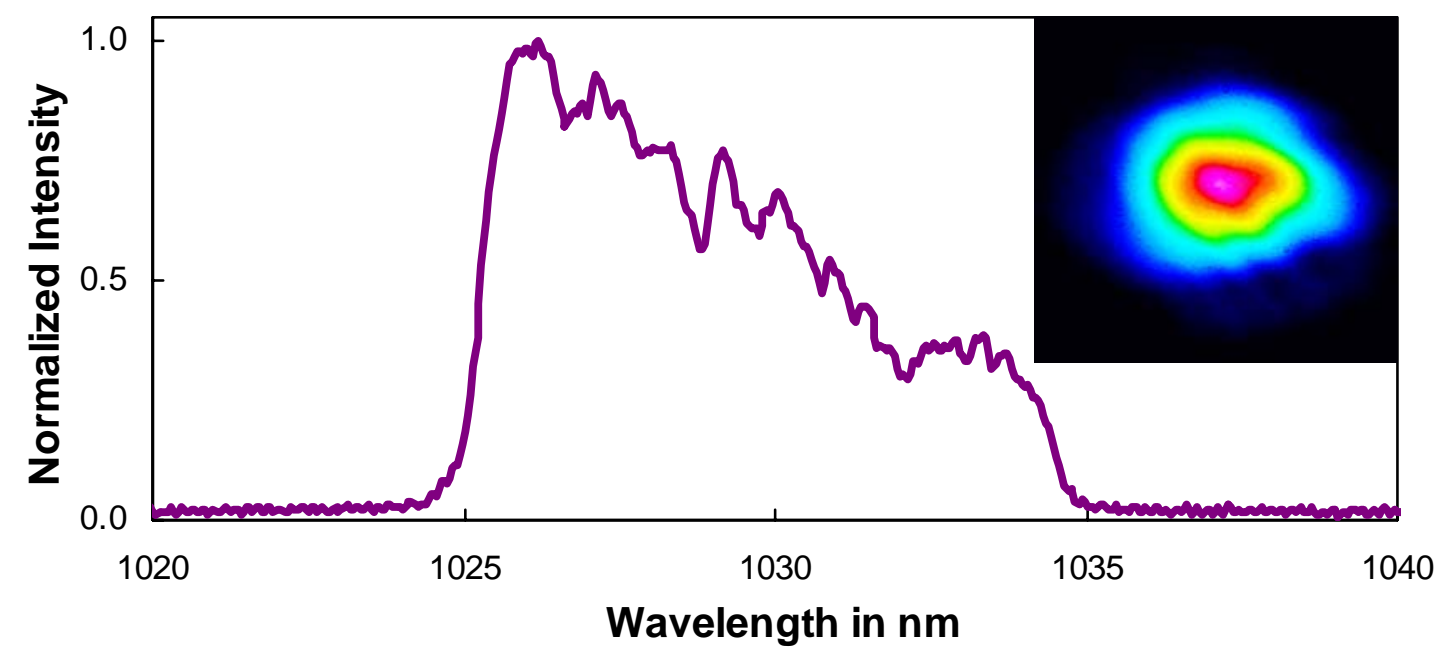

Fig. 6-18 Seed spectra provided by the Ti:sapphire oscillator together with the seed beam profile after the stretcher. The stretcher is optimized for a center wavelength of $1030 \mathrm{~nm}$, supports a bandwidth of $\sim 10 \mathrm{~nm}$ due to finite size of the used optical components and cuts all other wavelength off.

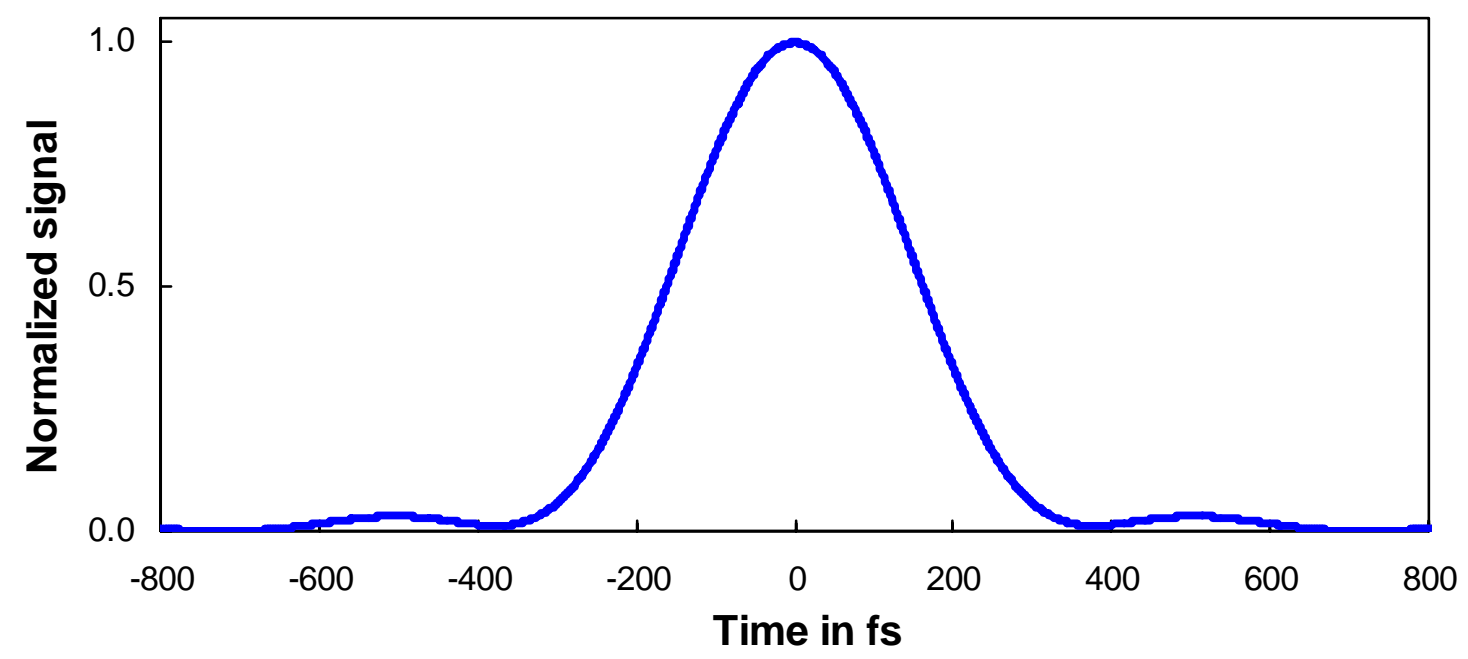

Fig. 6-19 Calculated Fourier-transform limited pulse duration of $230 \mathrm{fs}$ considering the spectra supported by the stretcher in figure 6-18.

After stretching, a rubidium titanyl phosphate (RTP) Pockels cell (PC) is used to slice pulses from the $\sim 70 \mathrm{MHz}$ oscillator pulse train at a frequency of $10 \mathrm{kHz}$. An additional isolator protects the RTP PC and the seed oscillator against feedback from the regenerative amplifier.

The same $20 \mathrm{~mm}$ short $\beta$-barium borate (BBO) crystal based PC as described earlier has been chosen in order to switch the selected pulses in and out of the regenerative amplifier cavity. Inside the regenerative cavity, pulses are amplified in the resonator shown in figure 6-16. Following amplification, recompression is performed using two transmission gratings with 1400 lines/mm arranged in a double pass Littrow-configuration $\left(\mathrm{GDD}=-1.44 \cdot 10^{7} \mathrm{fs}^{2}\right.$ ), with overall transmission of $\sim 70 \%$ as described in chapter 6.1 . 


\subsubsection{Plane-parallel disk}

The amplifier head from Trumpf Laser contains a $\sim 1 / 10 \mathrm{~mm}$ thick plane parallel Yb:YAG thin-disk mounted on a diamond heat sink, and is diode pumped with up to $500 \mathrm{~W}$ at a wavelength of $940 \mathrm{~nm}$ in a region smaller than $3 \mathrm{~mm}$ in diameter.

During amplification substantial gain narrowing is observed, resulting in shortening of the chirped pulse duration inside the amplifier cavity to already $\sim 70$ ps by the time the pulses reach only the energy of $0.24 \mathrm{~mJ}$. The SHG autocorrelation trace of the uncompressed pulse shows figure 6-20.

The reduced stretched pulse duration increases the possibility for nonlinear effects. To reduce these effects the number of roundtrips was minimized and the diode pump power was increased for higher single-pass gain and to reach higher pulse energies. Before compression, pulse energies as high as $8.0 \mathrm{~mJ}$ could be achieved.

The spectra of the pulses amplified to $2.0,4.0$ and $8.0 \mathrm{~mJ}$ are shown in figure $6-21$. The FWHM bandwidth of the pulses amplified to pulse energies of $8.0 \mathrm{~mJ}$ was measured to be $0.82 \mathrm{~nm}$ and smaller than the expected value around $1.0 \mathrm{~nm}$ taking the gain narrowing into account estimated in chapter 3.3. To give an impression about the effect of gain narrowing, the amplified pulse spectrum at $8.0 \mathrm{~mJ}$ is shown in figure 6-22 in comparison to the seed spectrum leaving the stretcher.

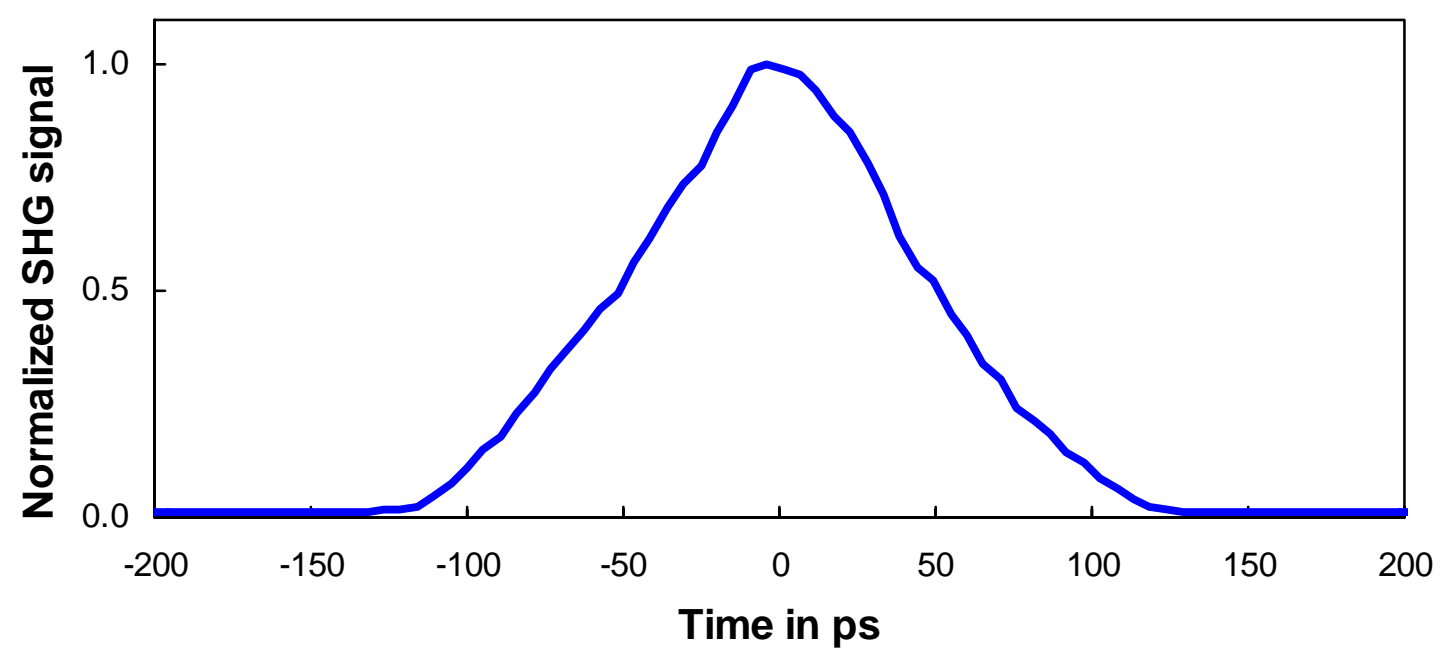

Fig. 6-20 Measured autocorrelation trace of the amplified pulse before compression at $0.24 \mathrm{~mJ}$ pulse energy and a repetition rate of $10 \mathrm{kHz}$.

The amplifier cavity supports only the $\mathrm{TEM}_{00}$ fundamental laser mode leading to a nearly diffraction-limited focused output beam of significantly improved spatial properties compared to the seed. The beam profile measured at the position of the Pockels cell at $8.0 \mathrm{~mJ}$ pulse energy is shown as inset in figure 6-21. 


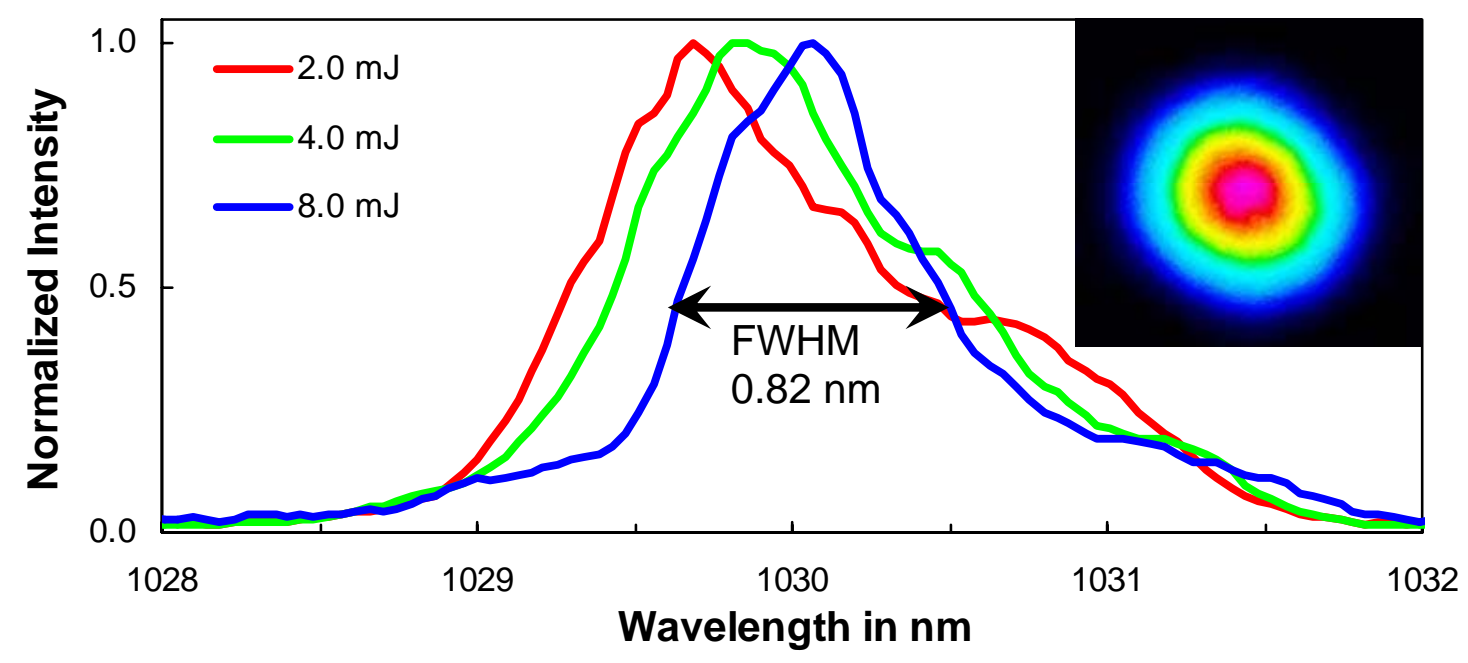

Fig. 6-21 Spectra of different energy levels of amplified pulses together with the beam profile measured at the position of the Pockels cell at an $8.0 \mathrm{~mJ}$ pulse energy at a repetition rate of $10 \mathrm{kHz}$. The FWHM bandwidth of the $8.0 \mathrm{~mJ}$ pulses is $0.82 \mathrm{~nm}$.

Figure 6-23 shows the measured SHG autocorrelation of the compressed output pulses at 2.0, 4.5 and $5.6 \mathrm{~mJ}$ at a repetition rate of $10 \mathrm{kHz}$. The measured pulse duration of $7.3 \mathrm{ps}$ (assuming a Gaussian temporal pulse shape) is much longer than the time-bandwidth limited pulse duration of $1.9 \mathrm{ps}$ to the corresponding measured spectral bandwidth of $0.82 \mathrm{~nm}$ for pulse energies of $5.6 \mathrm{~mJ}$ after the compression and could not be performed better neither by aligning nor changing the length of the compressor.

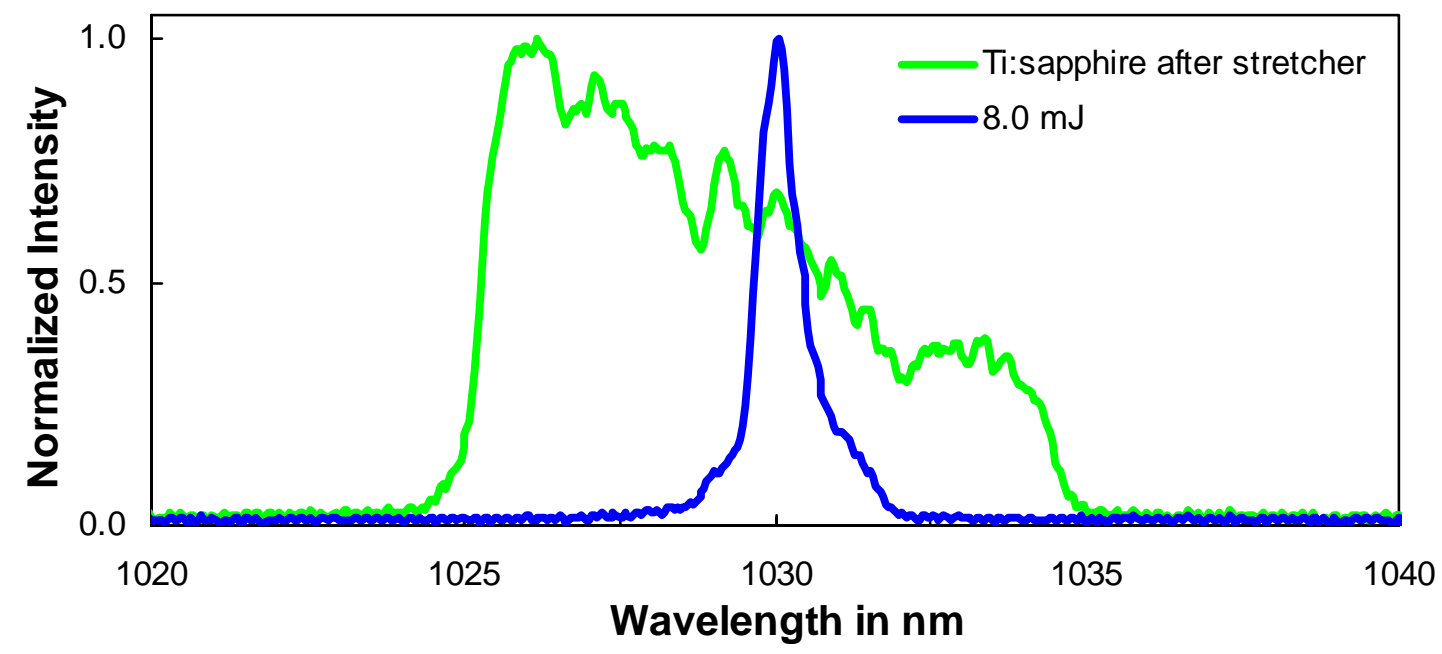

Fig. 6-22 Amplified pulse spectrum at $8.0 \mathrm{~mJ}$ compared to the seed spectrum.

Figure 6-24 shows the output power of the compressed pulses versus the pump power together with the chosen number of roundtrips in the amplifier cavity. At energy levels above $1.0 \mathrm{~mJ}$, bifurcation was observed and can be explained as follows. 


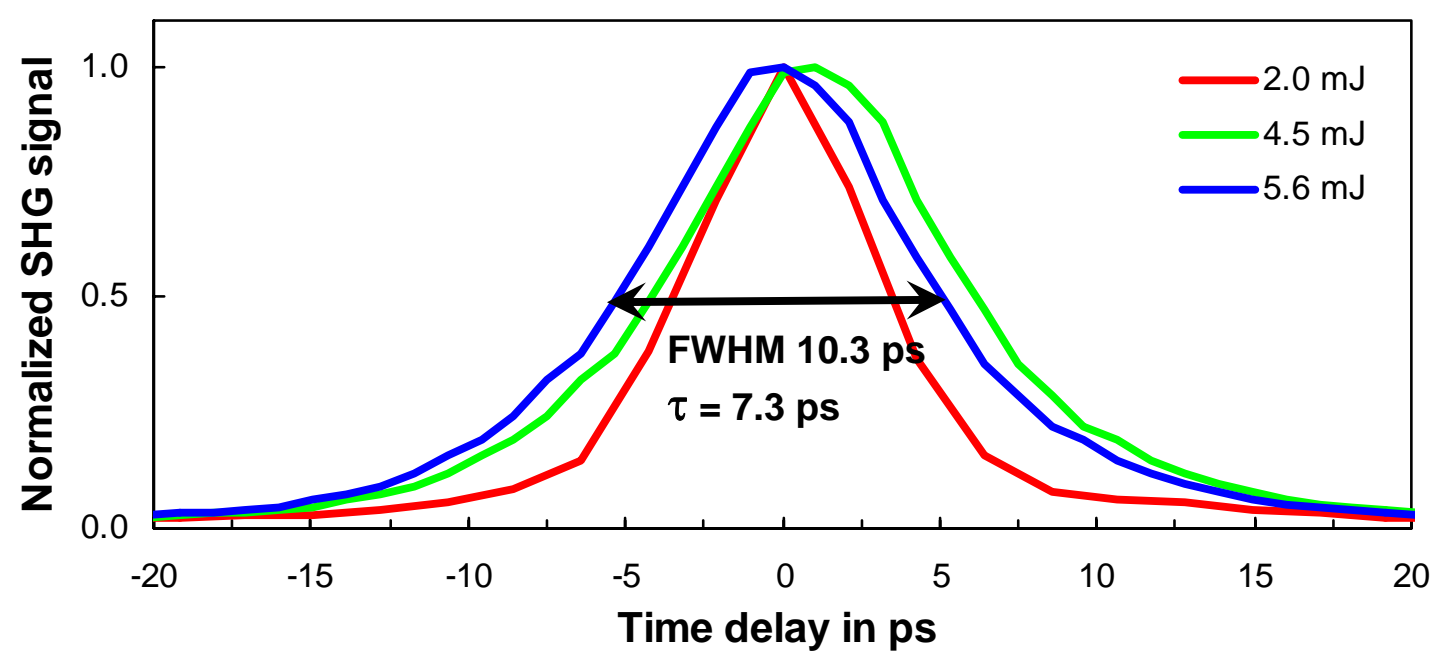

Fig. 6-23 Measured SHG autocorrelation of the compressed output pulses at 2.0, 4.5 and $5.6 \mathrm{~mJ}$ at a repetition rate of $10 \mathrm{kHz}$.

Due to amplification of the circulating pulses in the regenerative amplifier cavity the energy stored in the gain media is depleted by the pulses and by fluorescence. If the energy loss in the gain media is higher than the pump light can feed back into the disk between each amplification cycle, bifurcation occurs, leading to deterministic chaos and is described more detailed in chapter 7. As a result for example at the first bifurcation every second pulse experiences less amplification than the previous and is coupled out after the same number of round trips with less energy. To avoid bifurcation the number of round trips has to be reduced and the pump power needs to be increased [104]. The phenomena of bifurcation and chaotic pulse build up are described in detail in chapter 7. As a consequence of this phenomenon, the number of round trips had to be decreased to reach higher pulse energies accepting the high pump powers and an inefficient optical conversion from the pump light to the signal wavelength. The maximum achievable pulse energy was limited by the pump power. To reach an average output power of $56 \mathrm{~W}$ at a repetition rate of $10 \mathrm{kHz}$ after the compression, $500 \mathrm{~W}$ of pump power were required.

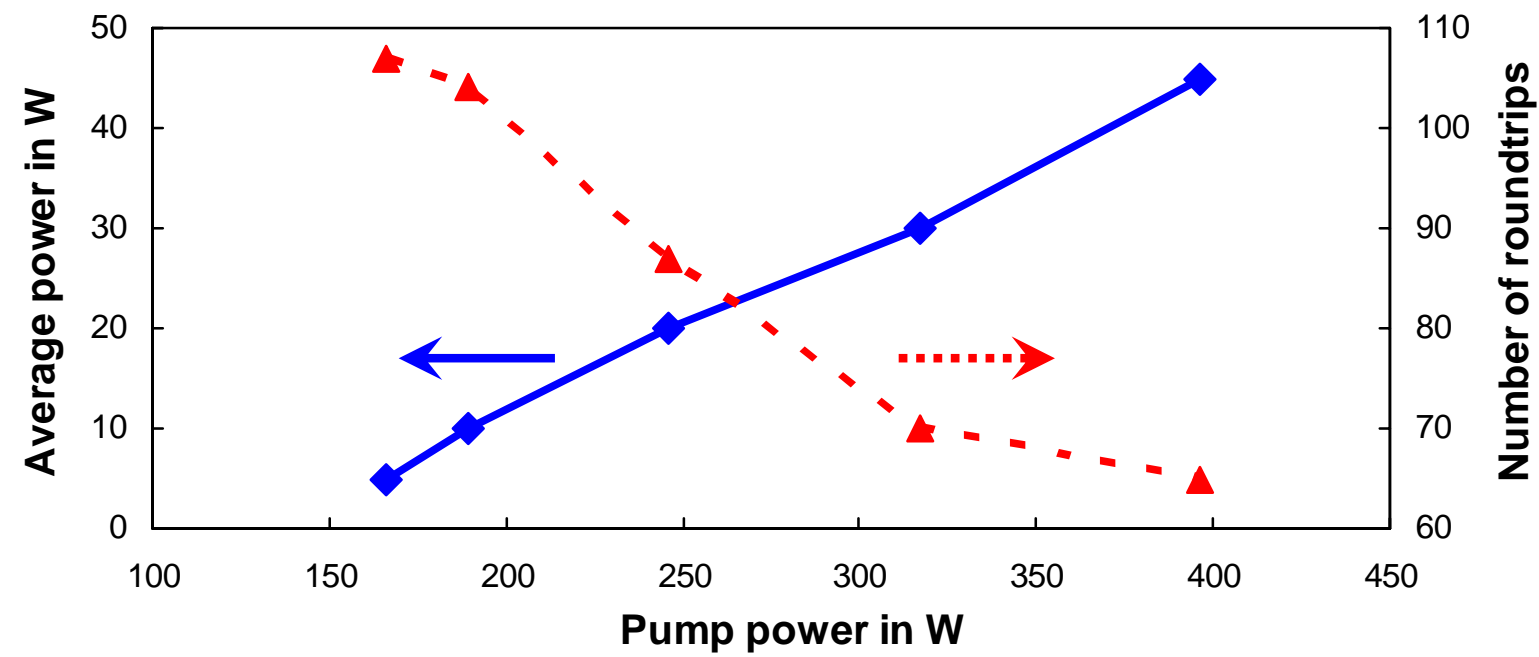

Fig. 6-24 Average output power and used number of cavity round trips versus the pump power at a repetition rate of $10 \mathrm{kHz}$. 


\subsubsection{Etalon effect of the plane disk}

The low measured bandwidth and the temporal incompressibility of the amplified pulses mentioned in the previous chapter gave an indication for a bandwidth limiting etalon effect in the system caused by the plane parallel Yb:YAG disk.

Intracavity etalons are commonly used in single frequency lasers to reduce the bandwidth [105] but etalon effects can also reduce the bandwidth in regenerative amplifier where single isolated pulses travel inside a cavity [106]. Etalon effects occur even for the anti-reflex coated Yb:YAG thin disk. The high amount of passes through the thin amplifier disk leads to a significant suppression of spectral components even for disks with only $0.1 \%$ reflection on the inner surface.

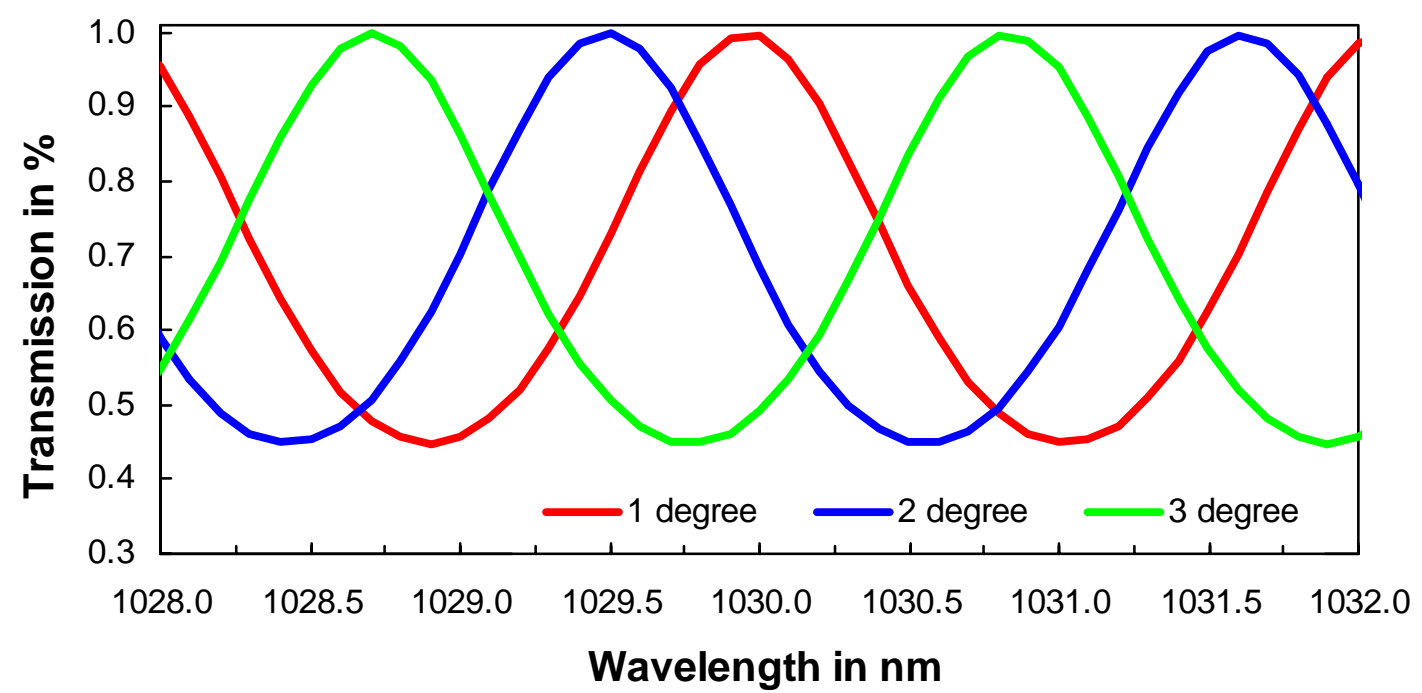

Fig. 6-25 Calculation of the overall optical transmission for incident angles of 1.0, 2.0 and 3.0 degree for 100 round trips in the amplifier resonator passing 200 times trough the disk in dependence of the wavelength.

The Yb:YAG disk can be examined as a Gires-Tournois interferometer [107] which is a special type of Fabry-Perot interferometer with one mirror having a reflection coefficient of almost one and is mathematically described in detail in [90]. Gires-Tournois interferometers are used amongst others for temporal pulse shaping of ps-pulses and find its application also in multi-layer mirrors [108].

Assuming a $136 \mu \mathrm{m}$ thin etalon corresponding approximately to the thickness of the disk with $99.9 \%$ transmission, the overall optical transmission for incident angles of 1.0, 2.0 and 3.0 degree for 100 round trips in the amplifier resonator passing 200 times trough the disk was calculated [109]. The calculation was done in dependence of the wavelength and of the interference between the front and the back surface of the disk and is presented in figure 6-25. The calculation shows that the transmitted bandwidth after amplification is drastically reduced and depends strongly on the incident angle leading to a variation in thickness of the disk. The free spectral range of the according etalon of $136 \mu \mathrm{m}$ thickness was calculated to be $\Delta \lambda=2.14 \mathrm{~nm}$.

Considering that the disk is used in reflection leads to a second etalon which can be assumed twice as thick as the disk with interference between the front surface and itself. In this case, 
the free spectral range is reduced by a factor of two and was calculated for a $272 \mu \mathrm{m}$ thick etalon to be $\Delta \lambda=1.07 \mathrm{~nm}$. Both etalons superpose and result in a heavy wavelength modulated transmission. The calculated transmission for a $136 \mu \mathrm{m}$ and a $272 \mu \mathrm{m}$ thick etalon illuminated under an angle of 1.0 degree and their superposition is shown in figure 6-26.

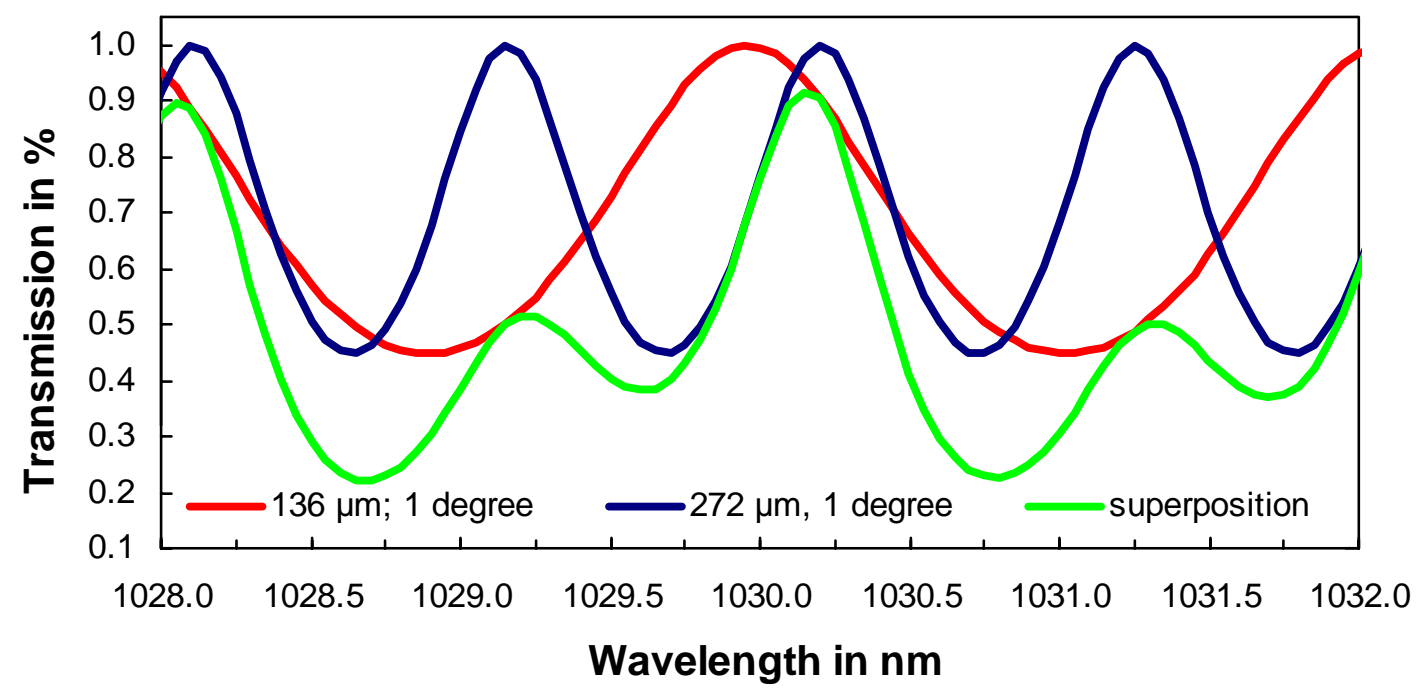

Fig. 6-26 Calculated transmission for a $136 \mu \mathrm{m}$ and a $272 \mu \mathrm{m}$ thick etalon illuminated under an angle of 1.0 degree and their superposition.

A complete wavelength and angle dependent gain characterization using the calculated transmission of the disk and the wavelength dependent gain cross section (the fluorescence of the disk around $1030 \mathrm{~nm}$ is depicted in figure 6-27) was not done at this point. However, the angle dependent reduction of bandwidth is obvious and the expected bandwidth of $1.0 \mathrm{~nm}$ due to gain narrowing is further reduced by the etalon effect of the Yb:YAG disk and results in the measured bandwidth of $0.8 \mathrm{~nm}$.

To investigate the etalon effect on the amplifier output spectrum, the angle of incidence on the $\mathrm{Yb}$ :YAG disk was changed as illustrated in figure 6-28. For each change, the cavity was newly aligned and the output spectrum of the amplified pulses at an energy level of $0.5 \mathrm{~mJ}$ at a repetition rate of $10 \mathrm{kHz}$ was measured for each angle.

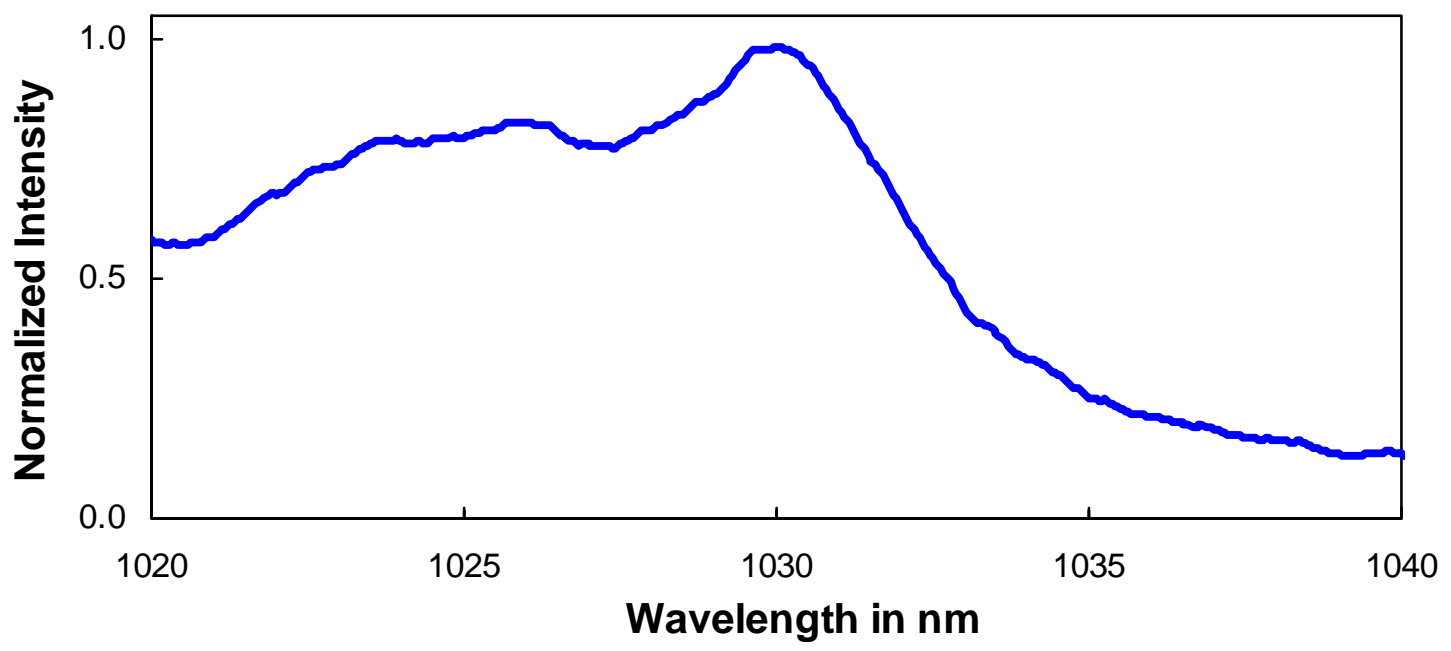

Fig. 6-27 Measured fluorescence distribution of the Yb:YAG amplifier disk. 


\section{regenerative amplifier}

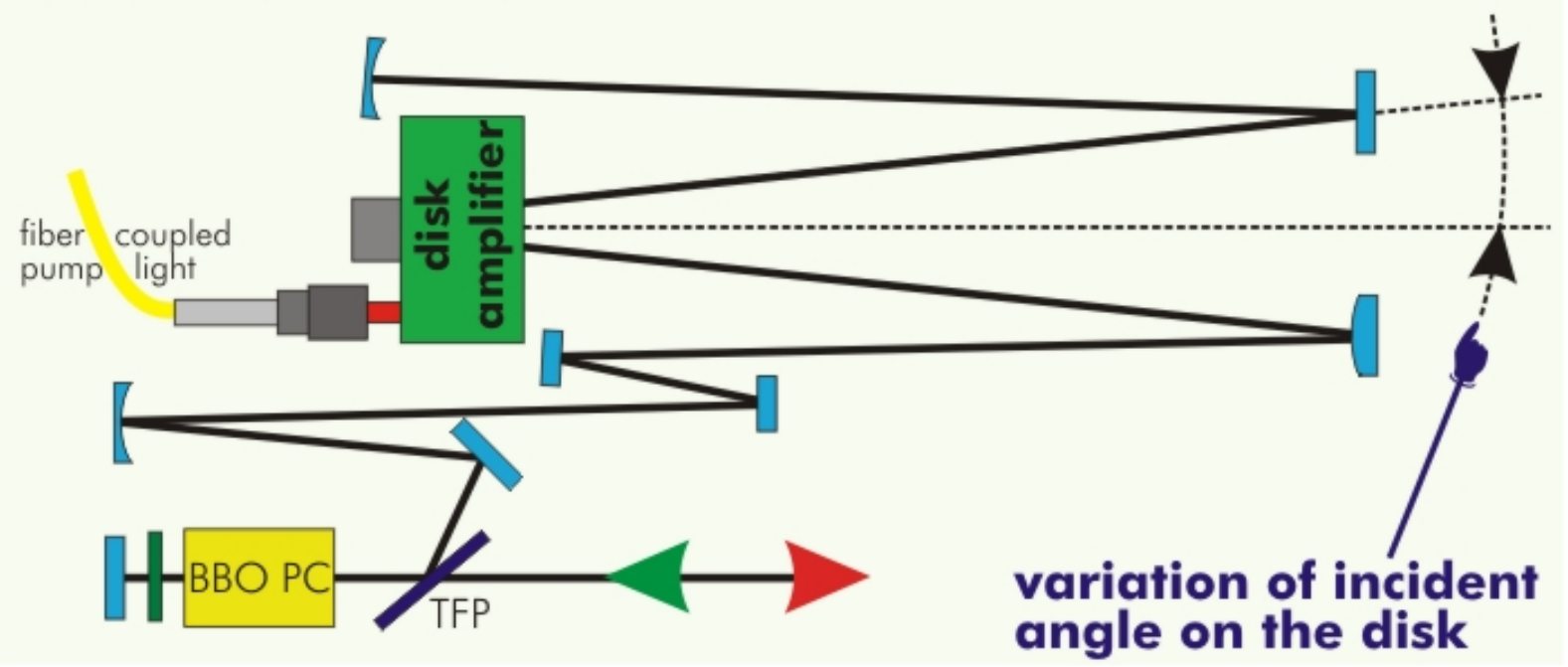

Fig. 6-28 Experimental setup to investigate the influence of the incident angle on the Yb:YAG amplifier thin disk. For each angular variation of the incident angle the amplifier resonator was realigned.

The anticipated influence of the Yb:YAG disk's angle of incidence on the output spectrum is indicated in figure 6-29. The dents in the spectral curve progression and the angle of incidence dependent spectral distribution indicate the etalon effect of the amplifier disk. The measurement was performed with low pulse energies of $0.5 \mathrm{~mJ}$ and therefore SPM can be excluded for causing the observed spectral modulation.

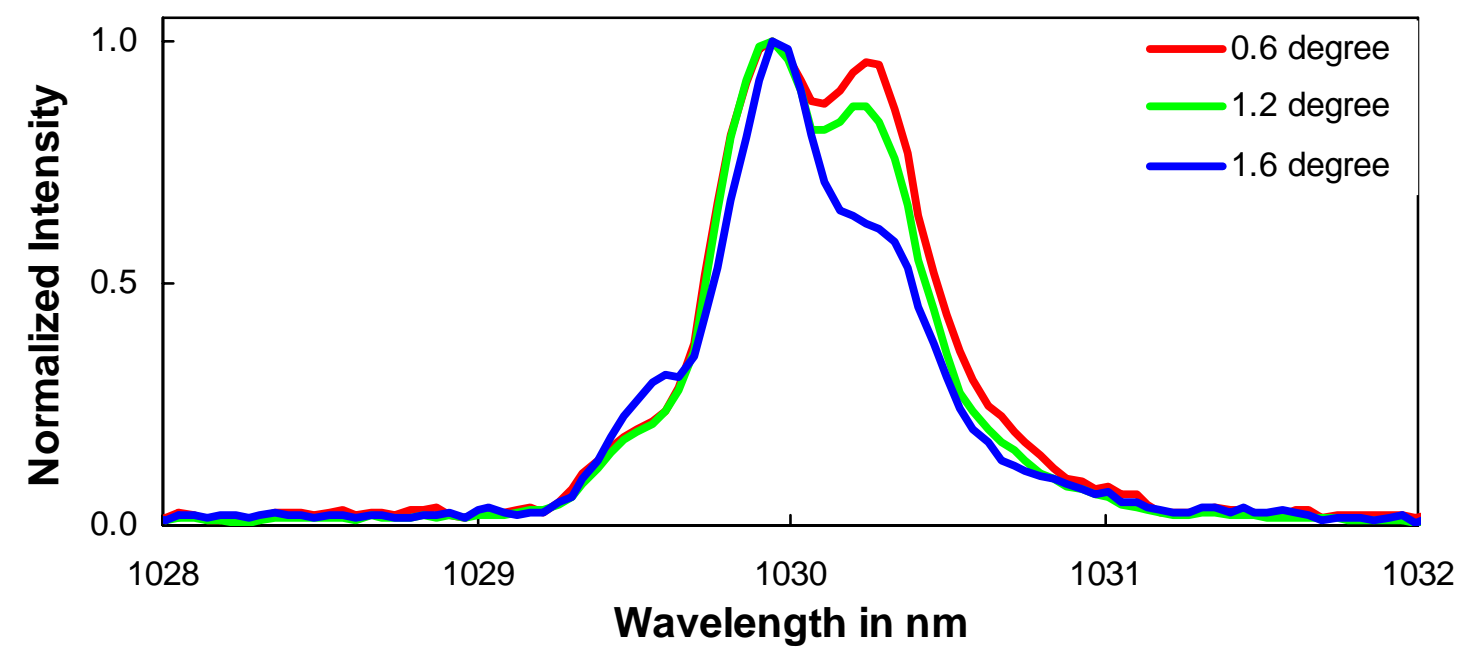

Fig. 6-29 Measured optical spectrum of the amplified pulses for three different angles of incidence on the Yb:YAG thin disk.

An effect on the compressed pulse duration could not be observed. Probably, the effect of multiple reflections from the disk surface due to imperfect anti-reflex coating and their amplification leading to successively temporal broadening with increasing number of round trips and the dispersion of the Yb:YAG disk caused Gires-Tournois interferometer are of much stronger influence on the pulse duration than the small spectral changes introduced by the varying angle of incidence. 
To avoid the negative effects of the plane parallel Yb:YAG amplifier thin disk on the minimum achievable pulse duration the disk was exchanged by a 0.1 degree wedged disk. The wedge guarantees that small reflections from the disk surface caused by imperfect antireflection coating don't remain inside the regenerative amplifier and impedes gain narrowing interference.

\subsubsection{Wedged disk}

In first experiments at low pulse energies, the performance of the wedged $\sim 1 / 10 \mathrm{~mm}$ thin $12.5 \%$ doped $\mathrm{Yb}$ :YAG disk and the compressibility of the amplified pulses was tested. Figure 6-30 shows the measured SHG autocorrelation and spectrum of the compressed output pulses at $1.0 \mathrm{~mJ}$ pulse energy at a repetition rate of $10 \mathrm{kHz}$.

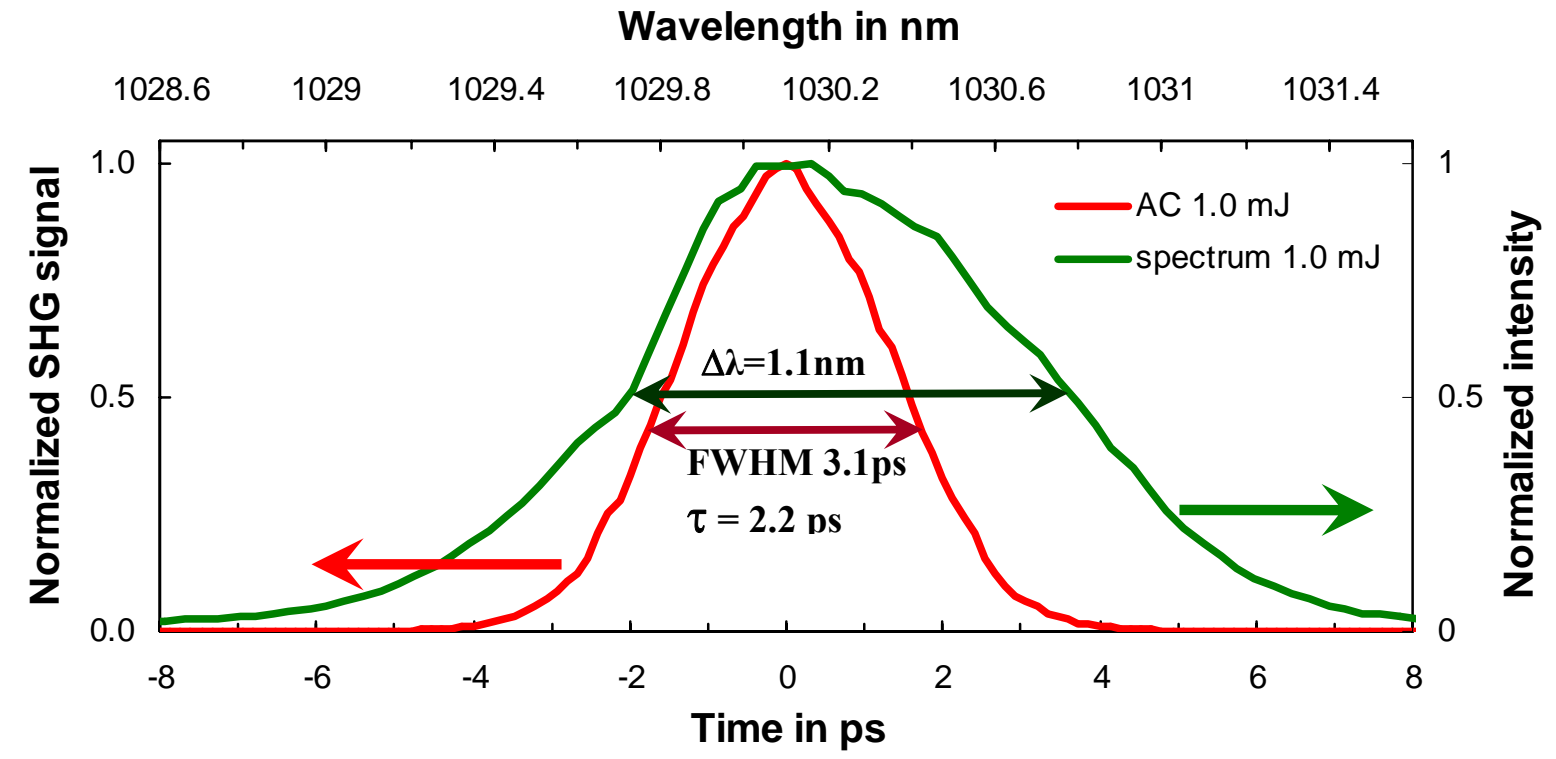

Fig. 6-30 Measured SHG autocorrelation and spectrum of the compressed output pulses at $1.0 \mathrm{~mJ}$ pulse energy at a repetition rate of $10 \mathrm{kHz}$.

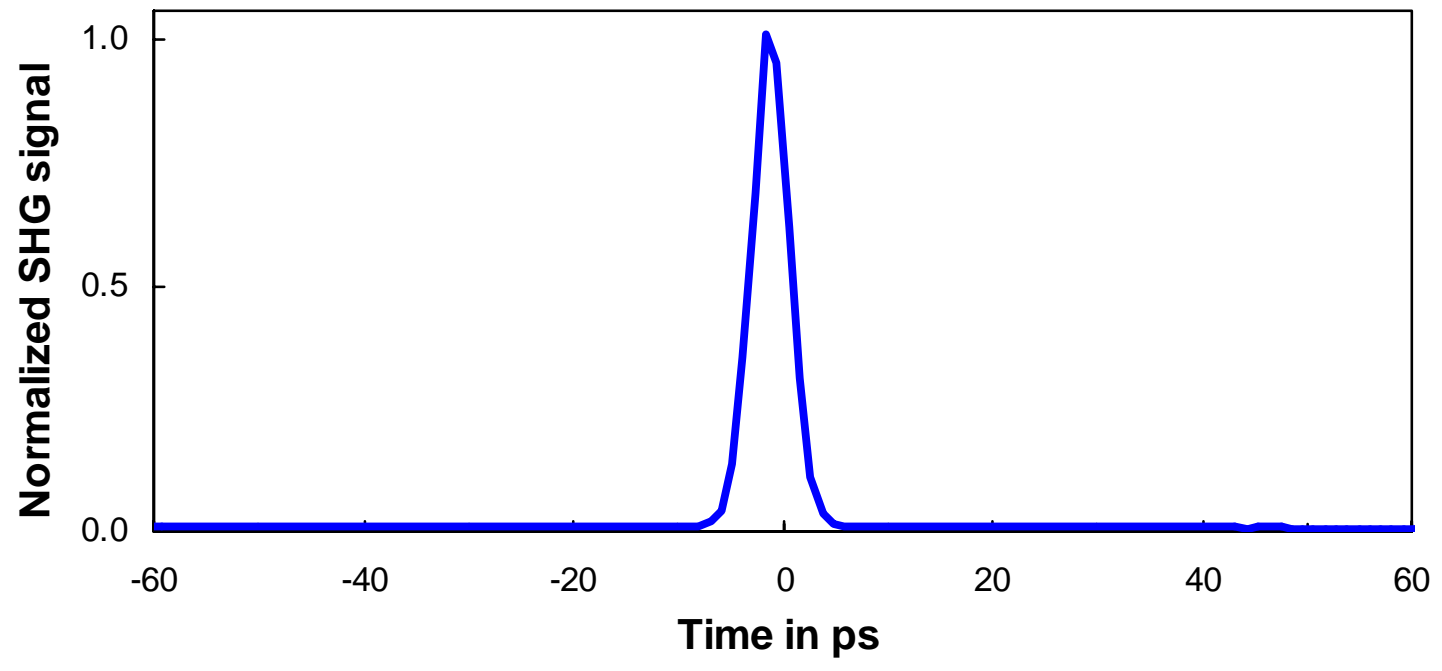

Fig. 6-31 Measured long range SHG autocorrelation of the compressed output pulse at $1.0 \mathrm{~mJ}$ pulse energy at a repetition rate of $10 \mathrm{kHz}$. No pre or post pulses could be observed. 
The measured spectral bandwidth is smooth and $1.1 \mathrm{~nm}$ wide corresponding to a time bandwidth limited pulse duration of $\sim 1.4$ ps. A first try of recompression led to pulse durations of $2.2 \mathrm{ps}$ (assuming a Gaussian shape) at a repetition rate of $10 \mathrm{kHz}$ and $1.0 \mathrm{~mJ}$ pulse energy. The measured pulse duration over a longer range of \pm 60 ps illustrated in figure 6-31 showed no side pulses, a clean autocorrelation trace and no Q-switch underground.

At this point, the compressor length was not completely optimized leading to the longer pulse duration of $2.2 \mathrm{ps}$ compared to the TBL duration of $1.4 \mathrm{ps}$.

Shortly mentioned before, bifurcation was again observed for higher pulse energies leading to pulse splitting in energy and even chaotic behavior for high numbers of round trips $>100$ and pump powers above $200 \mathrm{~W}$ and made clear at this point that a more comprehensive exploration of this phenomena is required.

Operating the regenerative amplifier at repetition rates near the inverse life time of the upper laser level and close to saturation in average power with low cw pump power and long temporal amplification cycles or more precisely with high numbers of round trips inside the amplifier resonator, pulse energy fluctuations and even chaotic pulse sequences of the out-coupled pulses can emerge [110]. For Yb:YAG with a lifetime of the upper laser level of $\sim 1 \mathrm{~ms}$ the multi-energy or chaotic pulse sequences occur for repetition rates between several $100 \mathrm{~Hz}$ and up to a few tens of $\mathrm{kHz}$. The dynamics of the output coupled energies are governed by the repetition rate, pump power, number of round trips, seed energy, fluorescence life time of the upper laser level and losses inside the cavity. All together they form a chaotic system which can be described as follows:

For a small number of round trips the circulating pulse cannot extract the whole stored energy from the amplifier gain medium, and a large fraction of the initial gain remains at the end of the amplification time giving the pump laser the chance to recover the gain until the next amplification cycle. However with increasing number of round trips the energy of the amplified pulse increases while the remaining gain in the laser medium decreases and cannot be recovered for a small initial gain (at low pump powers) to the previous value at the beginning of the amplification cycle. Therefore, this pulse is less amplified in the system than the previous; the system becomes unstable in terms of out coupled pulse energy leading to multiple bifurcations and even chaotic output energies [104]. Operating the laser in a chaotic, bistable or multi energy stable regime means that only the pulse energy is varying from pulse to pulse and not the out coupled average power witch always stayed stable within a maximum fluctuation of $\pm 0.5 \%$.

Figure 6-32 shows the photo diode signals (rise time $175 \mathrm{ps}$ ) of the out coupled pulses displayed via the fast acquisition mode (1000 pulses are displayed at the same time) of a $1 \mathrm{GHz}$ Tektronix oscilloscope and recorded for an observation time of $5 \mathrm{~ns}$ (left side) and $400 \mu \mathrm{s}$ (right side). In this operational mode shown in figure 6-32 an average power of $55 \mathrm{~W}$ after the compression at a PC repetition rate of $20 \mathrm{kHz}, 6.0 \mu \mathrm{s}$ amplification time corresponding to 174 round trips inside the resonator and $272 \mathrm{~W}$ pump power was achieved. In this single pulse splitting regime, every second pulse was developed only rudimentary to pulse energies of 
$\sim 0.69 \mathrm{~mJ}$ whereas the main pulses left the system with energies of $4.8 \mathrm{~mJ}$ at a repetition rate of $10 \mathrm{kHz}$ respectively.
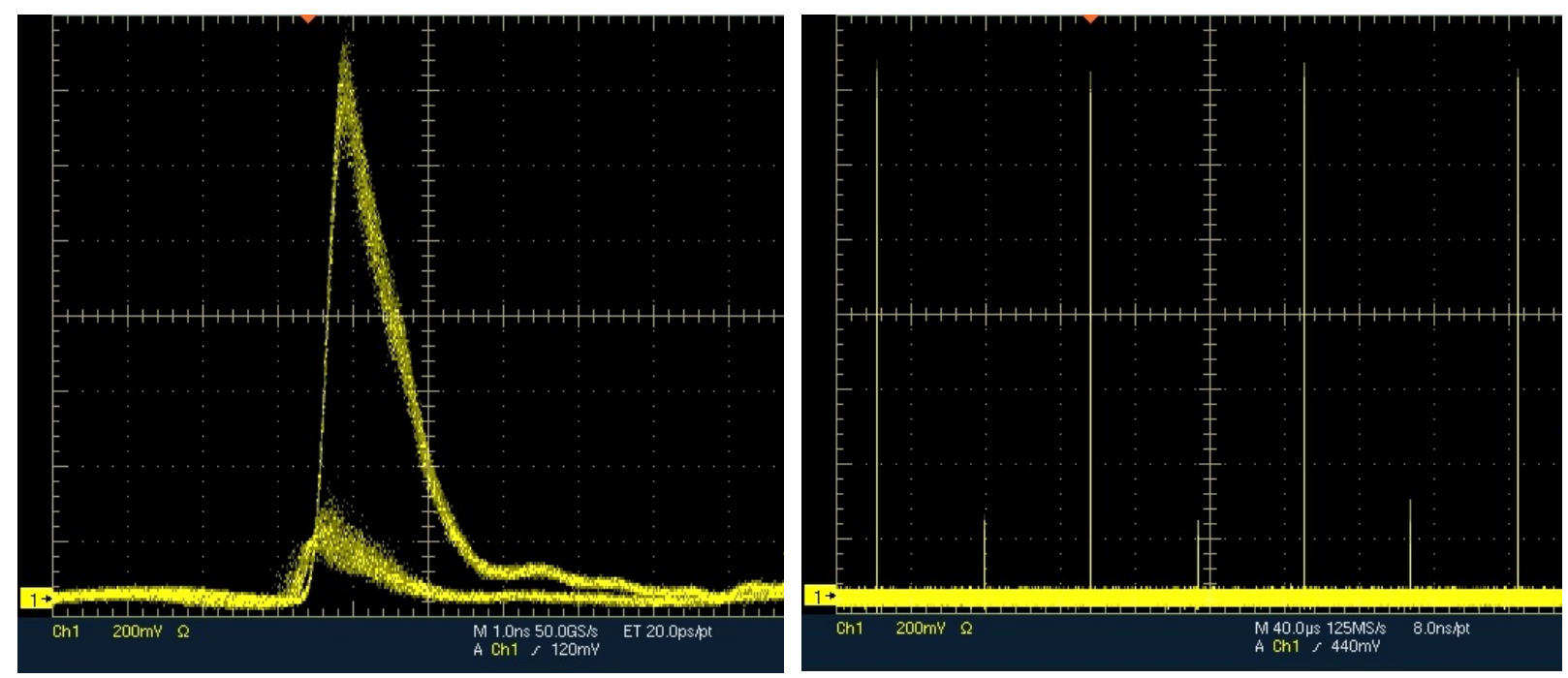

Fig. 6-32 Photo diode signals (rise time $175 \mathrm{ps)} \mathrm{of} \mathrm{the} \mathrm{out} \mathrm{coupled} \mathrm{pulses} \mathrm{displayed} \mathrm{via} \mathrm{the} \mathrm{fast} \mathrm{acquisition}$ mode (1000 pulses are displayed at the same time) of a $1 \mathrm{GHz}$ oscilloscope for an observation time of $5 \mathrm{~ns}$ (left side) and $400 \mu \mathrm{s}$ (right side). Repetition rate $20 \mathrm{kHz}, 6.0 \mu \mathrm{s}$ amplification time $=$ 174 round trips, $272 \mathrm{~W}$ pump power, $55 \mathrm{~W}$ of out coupled average power.

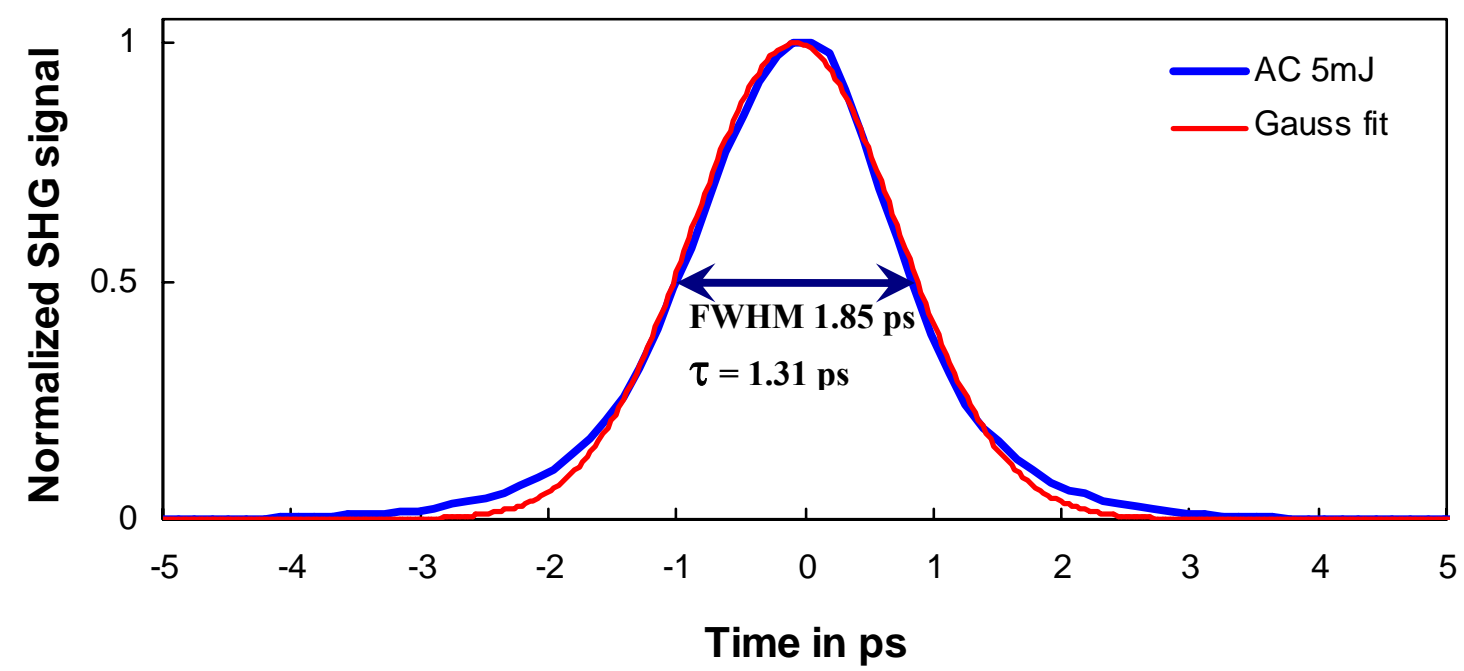

Fig. 6-33 Autocorrelation trace for the measured pulse splitting with a ratio of 7/1 between the main and the rudimentary pulse (meaning that $\sim 5 \mathrm{~mJ}$ are in the main pulse) at a repetition rate $20 \mathrm{kHz}$, $6.0 \mu$ s amplification time $=174$ round trips, $272 \mathrm{~W}$ pump power, $55 \mathrm{~W}$ of out coupled average power.

The pulses could be compressed to a duration of 1.31 ps assuming a Gaussian temporal shape. The measured autocorrelation trace of the $4.8 \mathrm{~mJ}$ pulses with a Gaussian fit is shown in figure 6-33 together with the corresponding spectra given in figure 6-34 compared to the spectra from figure 6-30 at $0.1 \mathrm{~mJ}$. Already at $5 \mathrm{~mJ}$ pulse energy slight spectral broadening from $1.1 \mathrm{~nm}$ at $1 \mathrm{~mJ}$ pulse energy to $1.6 \mathrm{~nm}$ (at $5 \mathrm{~mJ}$ pulse energy) occurred explaining the short pulse duration of $1.3 \mathrm{ps}$. 


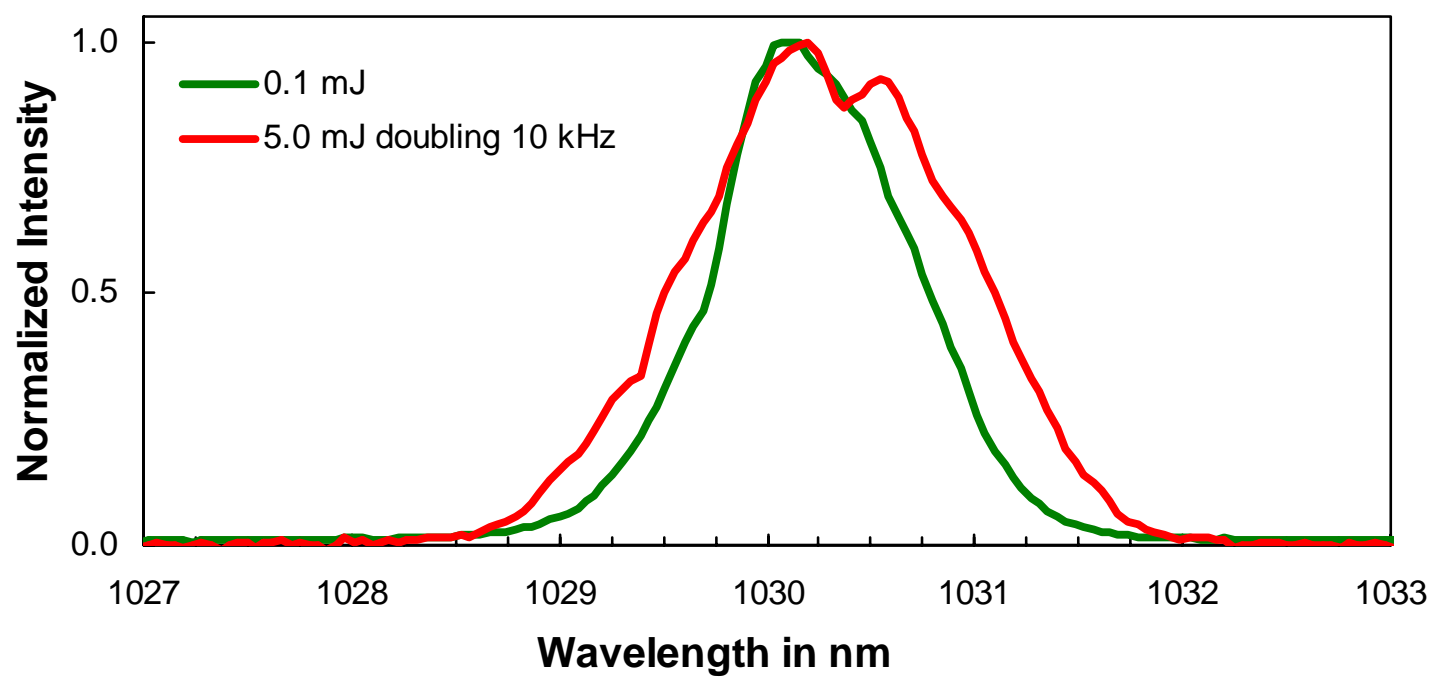

Fig. 6-34 Measured spectra for the pulse splitting ( doubling) with a ratio of 7/1 between the main and the rudimentary pulse (meaning that $\sim 5 \mathrm{~mJ}$ are in the main pulse) at a repetition rate $20 \mathrm{kHz}$, $6.0 \mu$ s amplification time $=174$ round trips, $272 \mathrm{~W}$ pump power, $55 \mathrm{~W}$ of out coupled average power.

A complete theoretic comprehensive description of stability features of continuously pumped high repetition rate regenerative amplifier based on laser media with long relaxation times is given in [111]. It was shown that in the chaotic pulse build-up of regenerative amplifiers single energy areas exist. So called intermitted stable regimes appear in the bifurcation driven deterministic chaotic pulse build up system of the cw pumped regenerative amplifier. Within this intermitted areas highly stable single energy regimes can be observed.

By varying the parameters affecting the bifurcation, i.e. repetition rate, pump power and number of round trips, such stable regimes could be found with the here presented regenerative amplifier. Figure 6-35 shows for example a stable period doubling of the output pulses in respect to the PC repetition rate where only every second pulse is amplified to full energy whereas every other $2^{\text {nd }}$ pulse completely disappears. At higher repetition rates also period tripling was observed.

Figure 6-35 shows the photo diode signal (rise time $175 \mathrm{ps}$ ) of the out coupled pulses displayed via the fast acquisition mode (1000 pulses are displayed at the same time) of a $1 \mathrm{GHz}$ oscilloscope for an observation time of $12.5 \mathrm{~ns}$ (left side) and $1000 \mu$ s (center \& right side). Clean period doubling could be observed for a PC repetition rate of $6.0 \mathrm{kHz}, 167$ round trips (5.8 $\mu$ s amplification time) and $302 \mathrm{~W}$ of pump power, resulting in $53 \mathrm{~W}$ of average power and a corresponding pulse energy of $17.7 \mathrm{~mJ}$ after the compression. The small double peaks in the picture in the center and right side derive from the high voltage switch of the PC inducing a signal into the BNC cable of the photodiode. Clean period tripling (right side) was observed for the $\mathrm{PC}$ repetition rate of $10.0 \mathrm{kHz}, 200$ round trips $(7.0 \mu \mathrm{s}$ amplification time $)$ and $445 \mathrm{~W}$ of pump power and resulted in $80 \mathrm{~W}$ of average power corresponding to pulse energies of $24.2 \mathrm{~mJ}$ after the compression. 

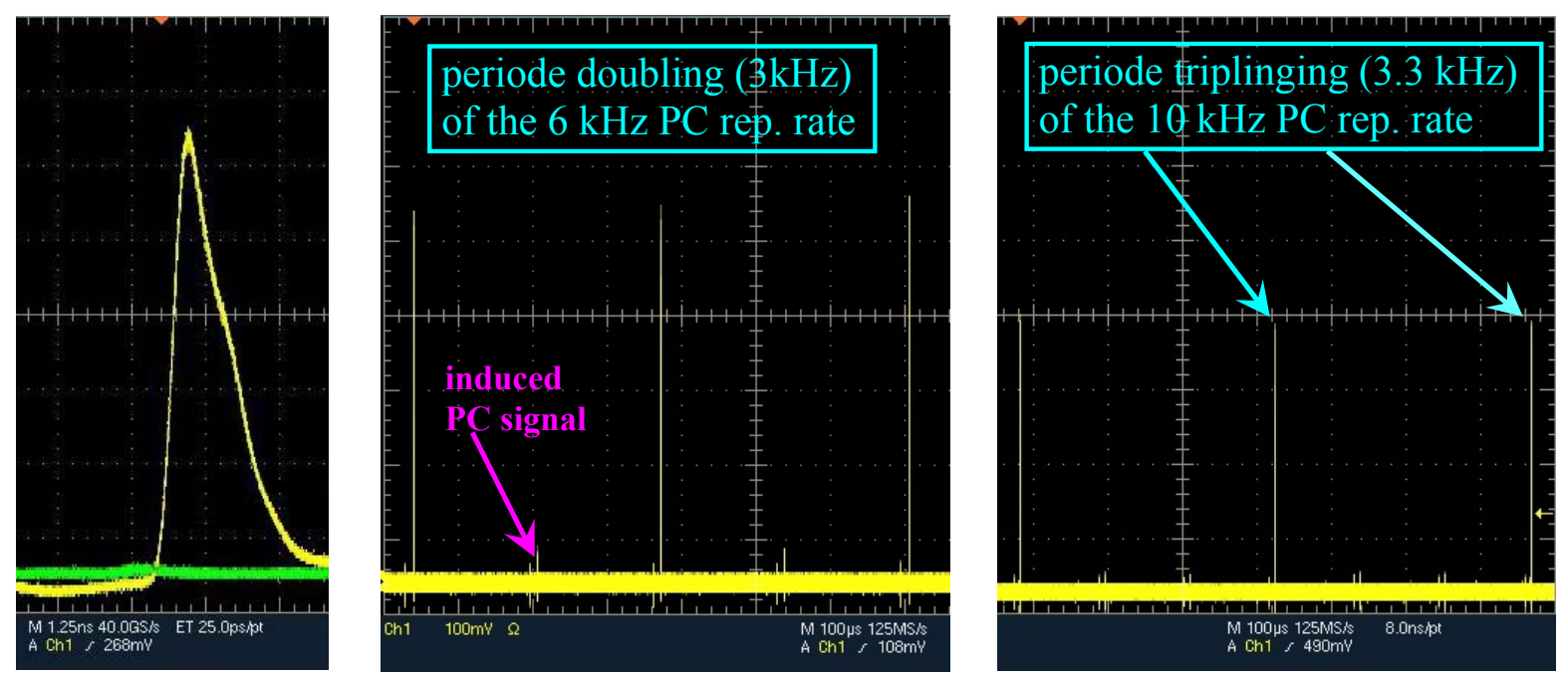

Fig. 6-35 Photo diode signals (rise time 175 ps) of the out coupled pulses displayed via the fast acquisition mode (1000 pulses are displayed at the same time) of a $1 \mathrm{GHz}$ oscilloscope for an observation time of $12.5 \mathrm{~ns}$ (left side) and $1000 \mu \mathrm{s}$ (center \& right side). Left side \& center: period doubling, $\mathrm{PC}$ repetition rate $6.0 \mathrm{kHz}, 167$ round trips, $302 \mathrm{~W}$ pump power, $53 \mathrm{~W}$ average power, pulse energy $17.7 \mathrm{~mJ}$. Right side: period tripling, PC repetition rate $10.0 \mathrm{kHz}, 200$ round trips, $445 \mathrm{~W}$ pump power, $80 \mathrm{~W}$ average power, pulse energy $24.2 \mathrm{~mJ}$

A more detailed description and the experimental results regarding the bifurcation, chaos, multi-and single-energy regimes are given in chapter 7 . The reachable pulse energies in the intermitted stable regimes are a multiple of the expected energy of $5.0 \mathrm{~mJ}$ and therefore SPM and SF made a longer stretching ratio of the seed pulses necessary before the amplification. At $24.2 \mathrm{~mJ}$ pulse energy, a B-integral of nearly 10 was accumulated and SF destroyed the PC crystal. Figure 6-36 and figure 6-37 show the measured spectra and selected measured autocorrelation traces for stable single pulse energy regimes with period doubling and tripling. The repetition rates mentioned in the legend is the resulting repetition rate of the out coupled pulses and not the switching frequency of the PC.

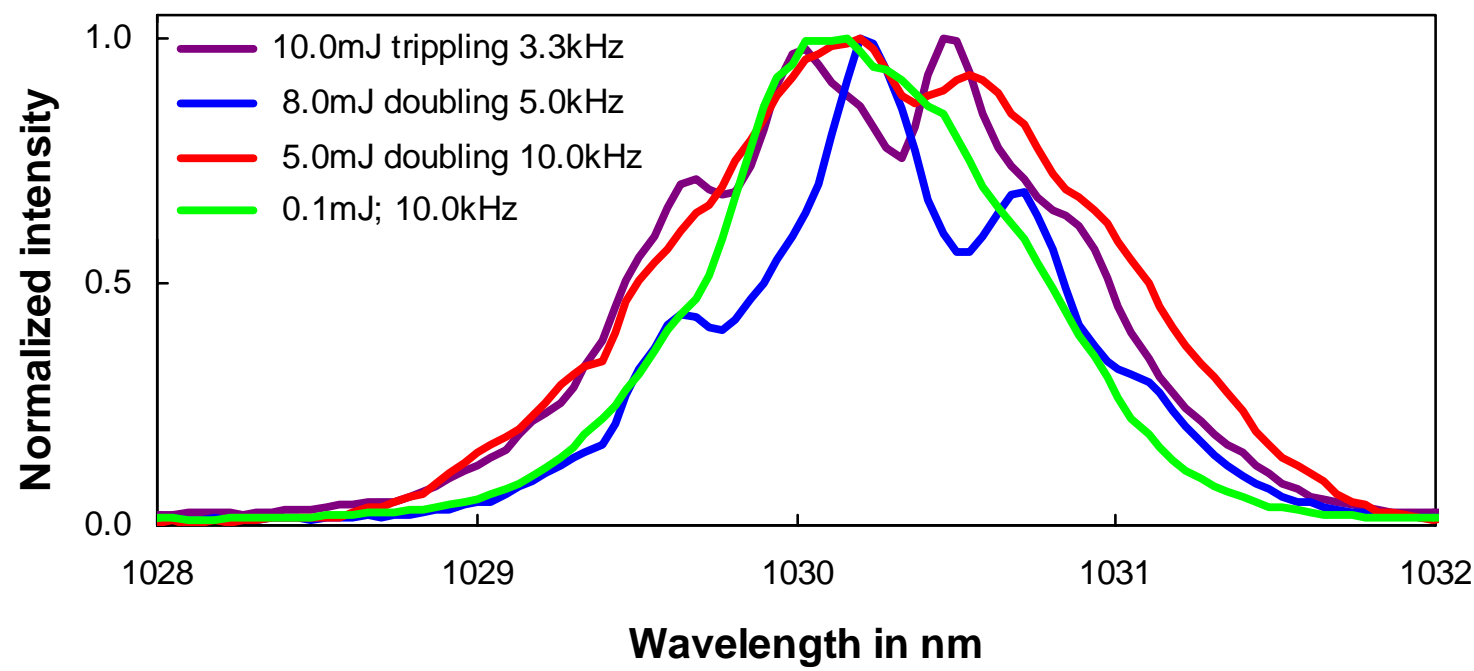

Fig. 6-36 Measured spectra for stable pulsed regimes with period doubling and tripling. The repetition rates mentioned in the legend is the resulting repetition rate of the out coupled pulses and not the switching frequency of the PC.

In regimes of complete period doubling or even tripling, the contrast between the two adjacent pulses coupled out by the PC at a given repetition rate is higher than a factor of 500 and from 
an standpoint of application it can be safely neglected, as the OPA gain scales as the exponential of the square root of the pump intensity [26]. Therefore it makes sense to mention only the repetition rate of the main pulses and not the PC switching frequency in stable single energy regimes.

In figure 6-36 the spectral broadening caused by SPM is clearly visible leading to an extreme temporal distortion of the pulses at energy levels above $24 \mathrm{~mJ}$ as shown in figure 6-37. Already at $6.2 \mathrm{~mJ}$ a small temporal elongation of the pulse duration occurred. Autocorrelations at 8.0 or $10.0 \mathrm{~mJ}$ could not be measured after the PC crystal was damaged due to SF. At $24.2 \mathrm{~mJ}$ tripling with $3.3 \mathrm{kHz}$ resulting repetition rate occurred with an average power of $80 \mathrm{~W}$ with 200 round trips and $445 \mathrm{~W}$ of pump power leading to heavy temporal distortions due to SPM which elongated the pulse duration and demand for even longer stretched seed pulses.

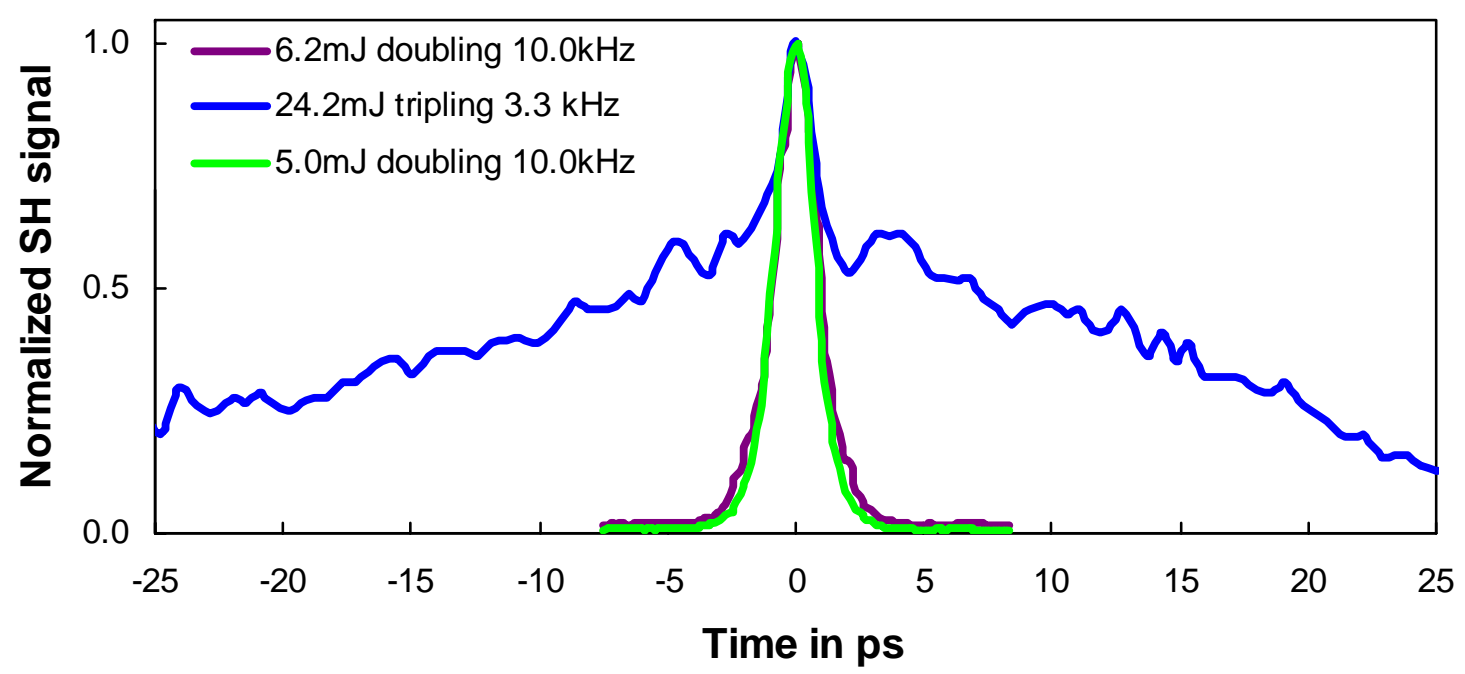

Fig. 6-37 Measured autocorrelation traces for stable pulsed regimes with period doubling and tripling. At $24.2 \mathrm{~mJ}$ (tripling with $3.3 \mathrm{kHz}$ resulting repetition rate, $80 \mathrm{~W}$ average power, 200 round trips and $445 \mathrm{~W}$ pump power) heavy temporal distortions due to SPM elongated the pulse duration and demand for even longer stretches seed pulses. 


\section{Regenerative amplifier based on the Trumpf Laser GmbH head with long stretcher and fiber preamplifier}

The pulse energies reached by making use of the intermitted stable single energy regimes mentioned in the chapter before were much higher than expected. Heavy SPM developed and SF caused bulk damage inside the PC BBO crystal requisitioning a longer temporal stretching of the seed pulses.

\subsection{Setup with stretcher and fiber preamplifier}

The stretcher used in the setup shown in figure 6-17 was changed to introduce a GDD of $1.185 \cdot 10^{8} \mathrm{fs}^{2}$ to the seed pulse as described in chapter 6.1 . To keep the required space on the optical table small, the almost $3 \mathrm{~m}$ long stretcher was folded as illustrated schematically in figure $7-1$.

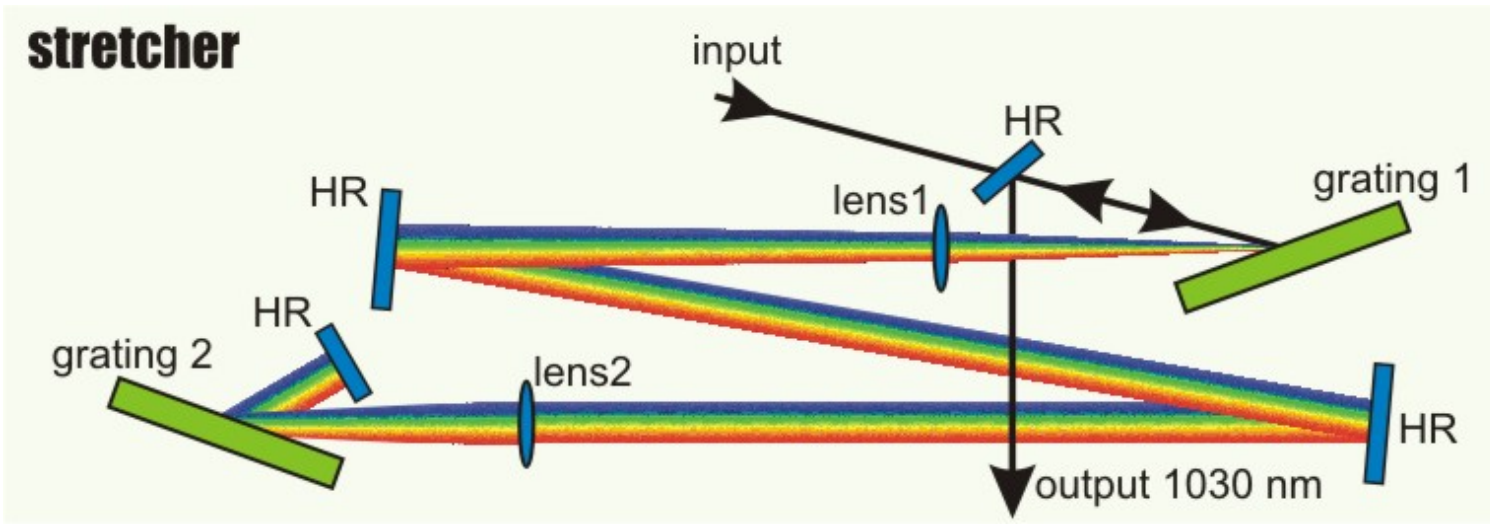

Fig. 7-1 Schematical pulse stretcher assembly introducing a positive group velocity dispersion of $1.185 \cdot 10^{8}$ fs $^{2}$ to the seed pulse.

The Ti:sapphire oscillator in the laboratory was not only used for delivering the seed pulses for the regenerative disk amplifier but also for other experiments and was positioned about $5 \mathrm{~m}$ away from the $\mathrm{Yb}$ :YAG amplifier setup. From time to time, the Ti:sapphire oscillator needed to be realigned or was optimized for other experiments leading frequently to a beam shift demanding for new alignment of the seed beam through the stretcher and the regenerative amplifier setup. Although the alignment was simplified with a series of pinholes it was extremely difficult to align the weak invisible infrared (IR) beam with infrared viewer and IR conversion cards through the entire amplifier setup including the exact position of the pump spot on the Yb:YAG disk especially after installing the new stretcher supporting even less bandwidth and energy than the previous shorter delay line. To simplify this alignment procedure and delivering higher seed energy with improved special properties compared to the IR Ti:sapphire seed beam, a $40 \mathrm{~dB}$ homebuilt double-stage fiber amplifier from the Friedrich Schiller University Jena was installed after the stretcher.

Using this approach allowed the alignment of the entire seed beam containing still all spectral components between $\sim 900$ and $1047 \mathrm{~nm}$ after the edge filter (introduced in figure 3-8) via two pinholes into the stretcher. After the stretcher acting as a spectral filter only a turning mirror was used to optimize the incoupling into the fixed positioned input of the fiber preamplifier. 
The output of the fiber was fixed at a permanent position making a daily realignment of the beam path after the fiber amplifier to the RTP pulse picker, mode matching telescopes, PC and amplifier resonator obsolete. Once the resonator cavity was precisely overlapped with the seed after the fiber preamplifier it had never be realigned again.

Figure 7-2 shows the spectra provided by the Ti:sapphire seed after the stretcher. The stretcher (figure 7-1) is optimized for a center wavelength of $1030 \mathrm{~nm}$, supports due to its finite size of the used optical components a bandwidth of $\sim 4 \mathrm{~nm}$ and cuts all other wavelength off and results in the steep edges of the supported spectra. The maximum seed energy after the stretcher within the $\sim 4 \mathrm{~nm}$ bandwidth remains between $700 \mathrm{fJ}$ and $1.3 \mathrm{pJ}$ depending on the alignment of the Ti:sapphire oscillator.

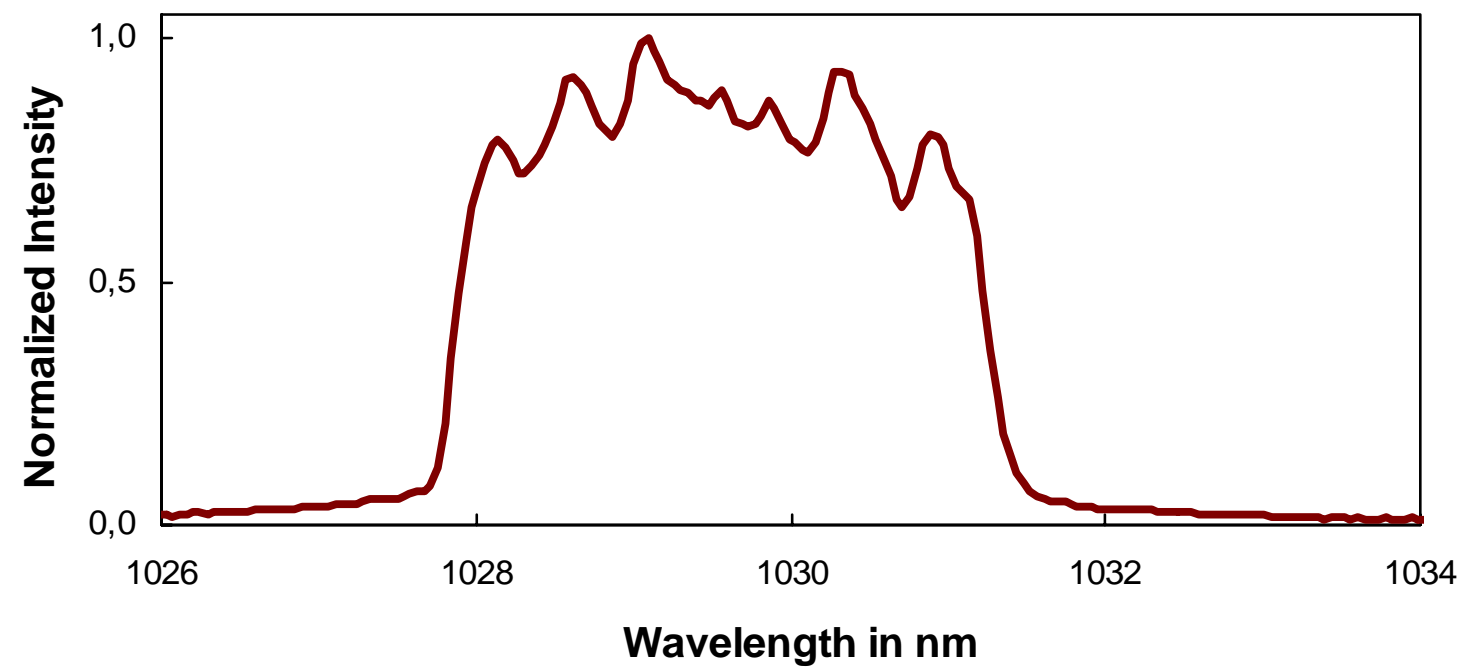

Fig. 7-2 Ti:sapphire provided seed spectra after the stretcher. The stretcher is optimized for a center wavelength of $1030 \mathrm{~nm}$, supports due to finite size of the used optical components a bandwidth of $\sim 4 \mathrm{~nm}$ and cuts all other wavelength off.

After the stretcher, a cylindrical lens telescope shapes the oval beam followed by a half-wave plate choosing the right input polarization before it is focused into the fiber preamplifier using a $400 \mathrm{~mm}$ focal lens. After amplifying the seed by $40 \mathrm{~dB}$ a lens recollimates the output followed by a half-wave plate together with a polarizer to cut off the depolarized light after the preamplification. The schematic setup of the fiber preamplifier is shown in figure 7-3.

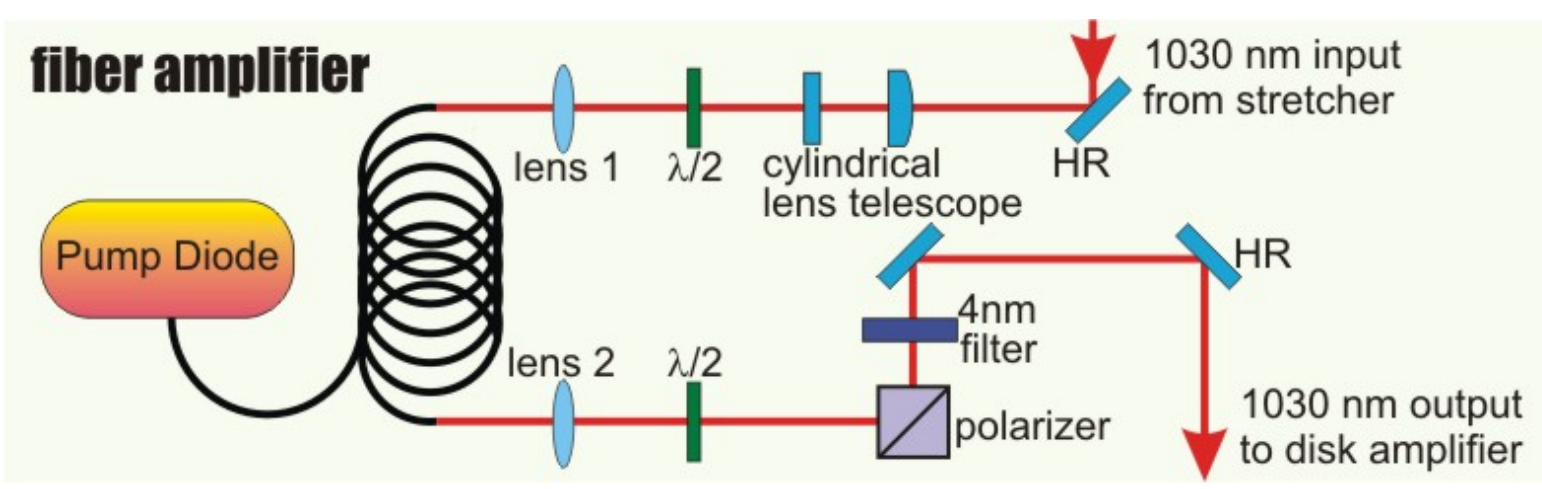

Fig. 7-3 Schematic setup of the $40 \mathrm{~dB}$ fiber preamplifier.

Figure 7-4 shows the fluorescence spectra of the fiber preamplifier at $890 \mathrm{~mA}$ pump current compared to the seed spectra supported by the stretcher and the output spectra after the fiber 
preamplifier seeded with $\sim 1 \mathrm{pJ}$. The remaining spectral bandwidth after the fiber preamplification is down to $\sim 1.6 \mathrm{~nm}$ but still broad enough to support TBL pulse durations of $<1 \mathrm{ps}$ and therefore still satisfies the demands of pulse durations $<2$ ps after the recompressed regenerative amplifier output. Figure 7-5 shows the beam profile of the stretched seed laser on the left side. The compressor was fully compensated for special chirp but showed nevertheless an elliptical profile which had to be corrected with a cylindrical lens telescope to couple it properly into the fiber amplifier. The corrected beam profile is shown in the center of figure 7-5. The right side shows the beam profile after the fiber amplifier at the position of the Pockels cell. Clearly visible is the improved beam profile compared to the low energy seed beam after the stretcher allowing good mode matching with the regenerative amplifier resonator.

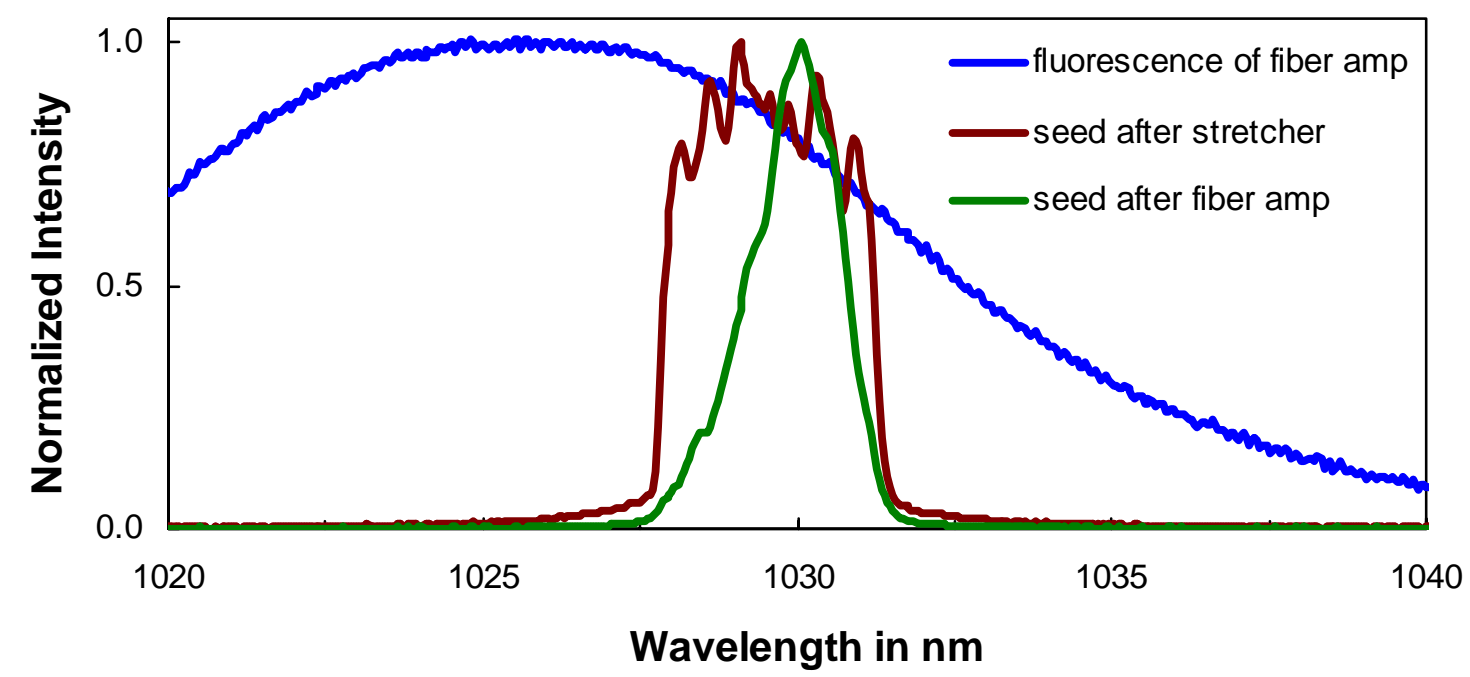

Fig. 7-4 Fluorescence spectra of the fiber amplifier compared to the seed spectra after the stretcher and the preamplified output spectra of the fiber amplifier. Measured for $1 \mathrm{pJ}$ total seed energy of the fiber amplifier pumped with $890 \mathrm{~mA}$.
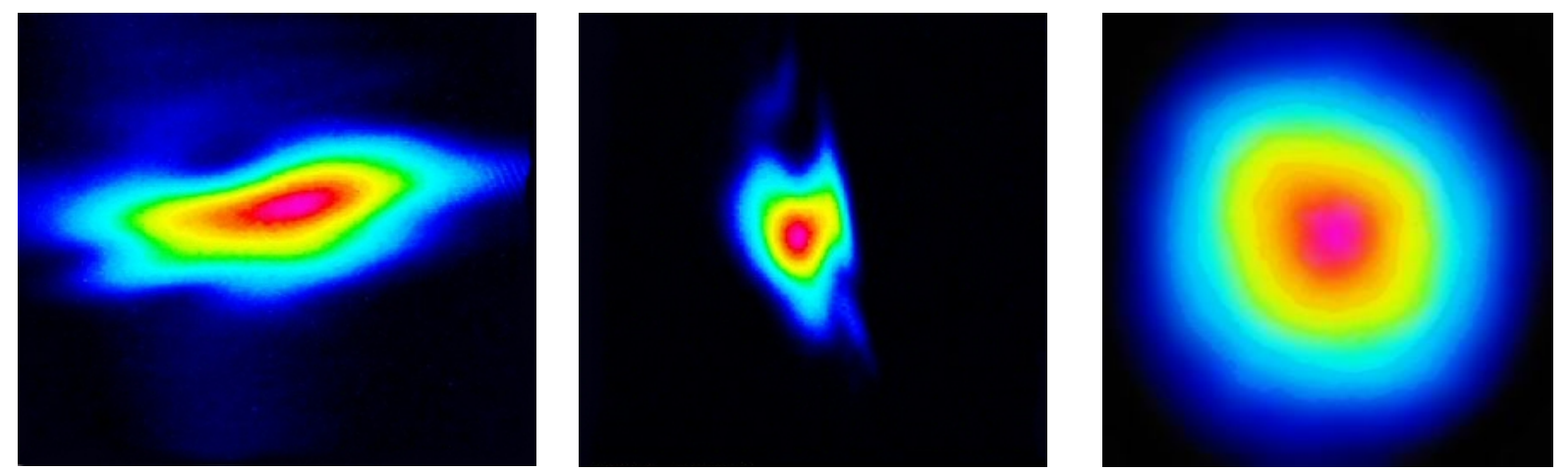

Fig. 7-5 Beam profiles in comparison after the stretcher (left side), after the cylindrical lens telescope before focusing it into the fiber preamplifier (center) and after switching the preamplified beam into the regenerative amplifier via the PC (right side).

Figure 7-6 shows the output power of the fiber preamplifier for 123 and $42 \mu \mathrm{W}$ of average seed power versus the pump power and pump current respectively. The low seed power of $42 \mu \mathrm{W}$ corresponds to the power obtained after the long stretcher with the spectrum shown in figure 7-2 leading to a maximum output power of $54 \mathrm{~mW}$ after the fiber preamplifier. Figure 7-7 depicts the output power of the fiber preamplifier versus the seed power of the setup shown in figure 7-3 at $890 \mathrm{~mA}$ pump current corresponding to $\sim 490 \mathrm{~mW}$ pump power. Fluo- 
rescence background was measured to be $\sim 3 \mu \mathrm{W}$ without seed. The green curve measured with an average seed power of $123 \mu \mathrm{W}$ corresponds to a larger bandwidth supported by the short stretcher shown in figure 6-18. At this point, the fiber preamplifier was not used in the setup.

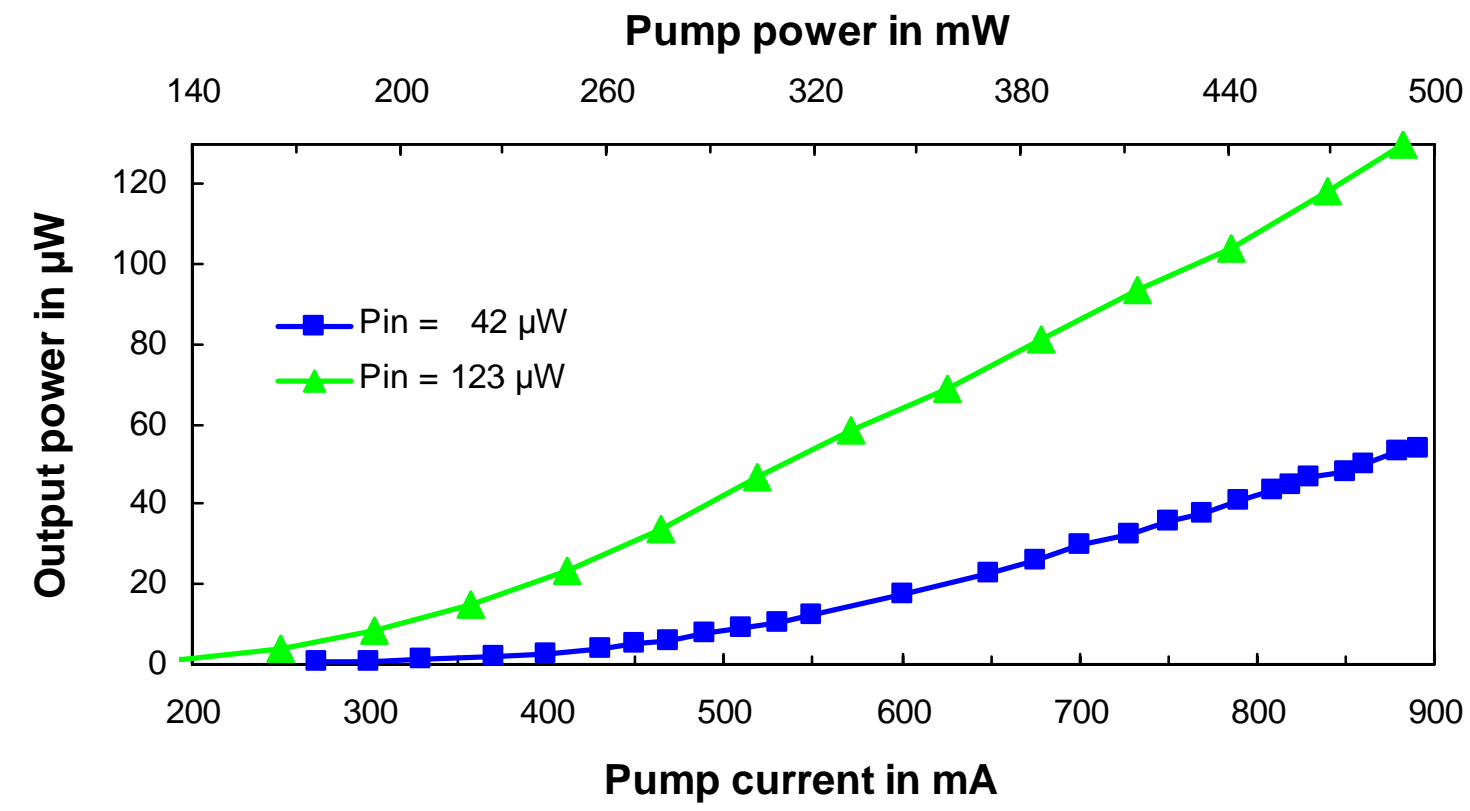

Fig. 7-6 Output power of the fiber preamplifier for 123 and $42 \mu \mathrm{W}$ of average seed power versus the pump power and pump current respectively. The low seed power of $42 \mu \mathrm{W}$ corresponds to the power obtained after the long stretcher with the spectrum shown in figure 7-2 leading to a maximum output power of $54 \mathrm{~mW}$ after the fiber preamplifier.

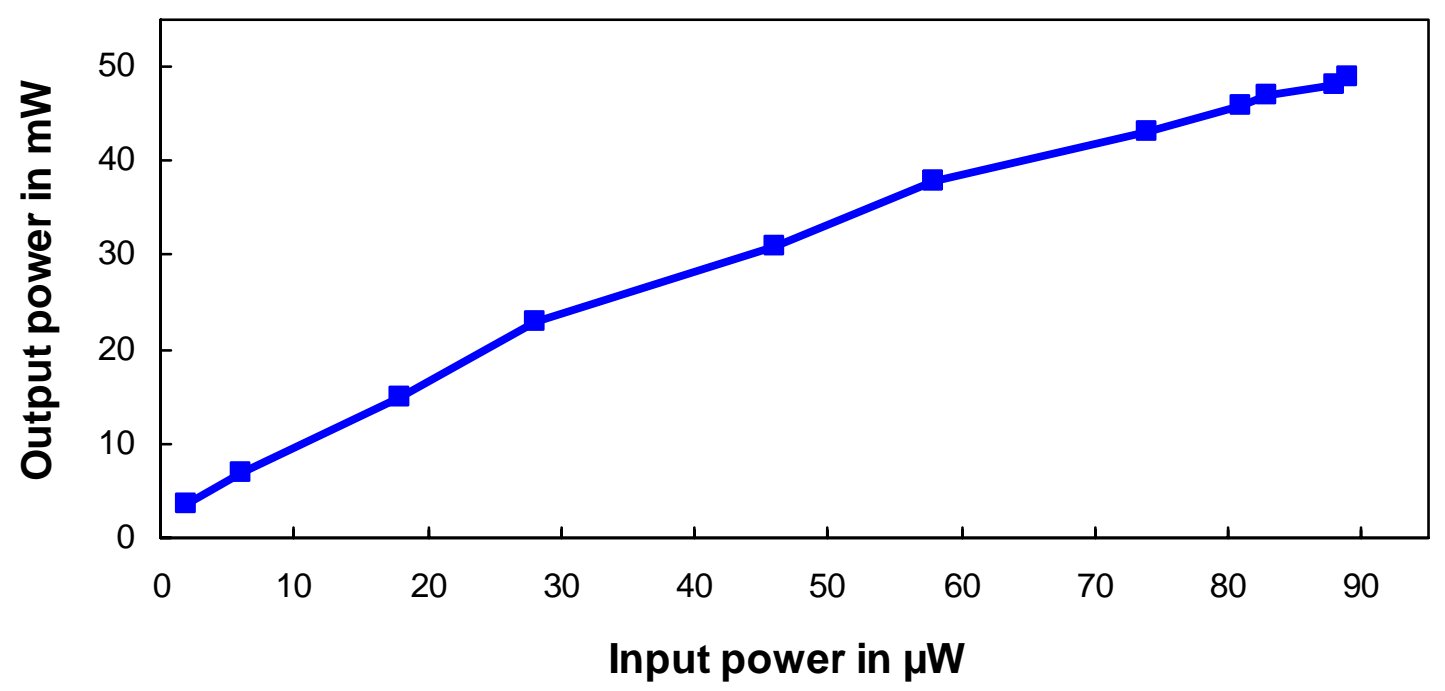

Fig. 7-7 Average output power of the fiber preamplifier versus seed power of the setup shown in figure 7-3 at $890 \mathrm{~mA}$ pump current corresponding to $\sim 490 \mathrm{~mW}$ pump power. Fluorescence background was measured to be $\sim 3 \mu \mathrm{W}$ without seed.

To check the compressibility of the pulses amplified in the fiber preamplifier and to choose the right input and output polarization of the fiber amplifier the seed pulses were directly compressed without using the regenerative amplifier. Figure 7-8 shows the measured autocorrelation trace of the recompressed pulses directly out of the fiber amplifier for three different chosen output polarization selected with the half wave plate (positions at 107, 120 and 140 
degree) and the polarizer after the fiber preamplifier. The minimum pulse duration obtained was 1.9 ps. In case of a wrong chosen polarization side peaks $\sim 7$ ps away from the main peak appear. At this point the input polarization had not been chosen correctly causing the broad pedestal of the pulses and was later corrected for the amplified pulses out of the regenerative system.

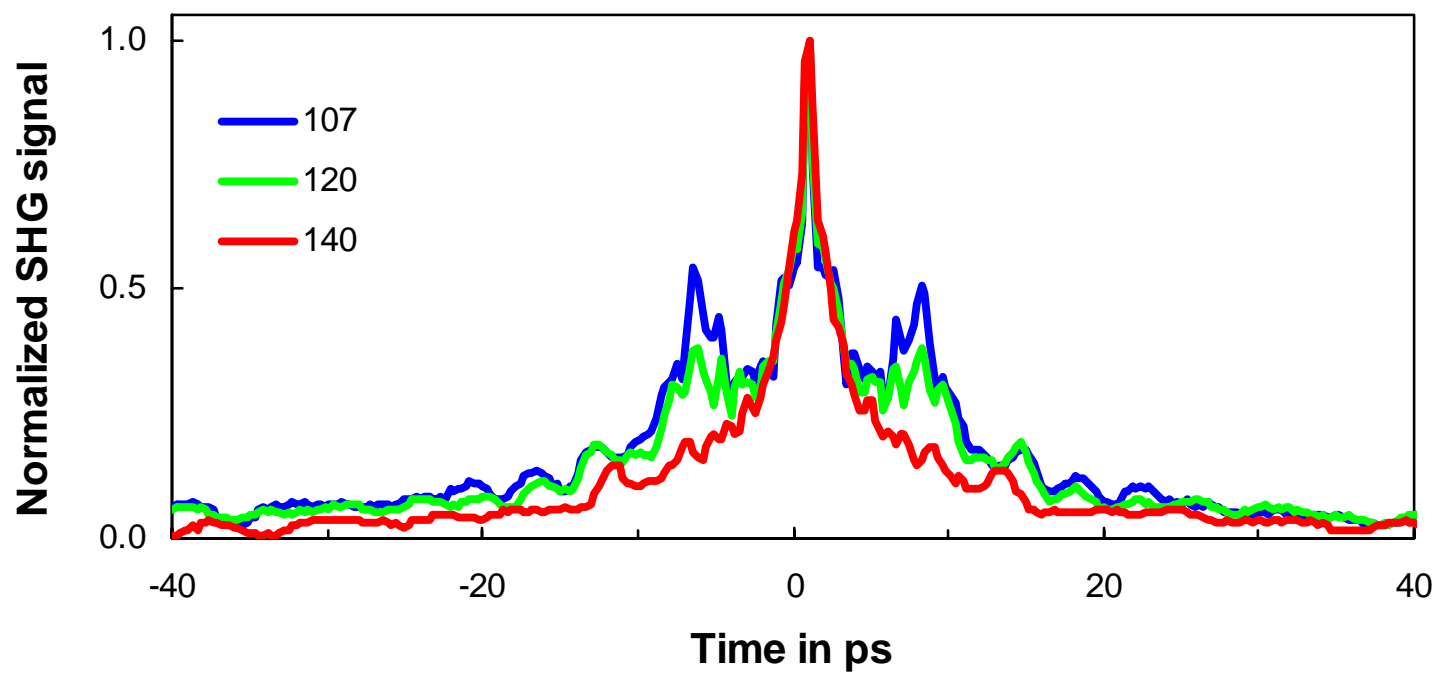

Fig. 7-8 Measured autocorrelation trace of the recompressed pulses directly out of the fiber amplifier for three different chosen output polarization selected with a half-wave plate (positions at 107, 120 and 140 degree) and the polarizer after the fiber preamplifier. The minimum pulse duration obtained was $1.9 \mathrm{ps}$. In case of a wrong chosen polarization side peaks $7 \mathrm{ps}$ away from the main peak appear.

The complete final setup consists of a seed laser, stretcher, fiber preamplifier, pulse picker, amplifier resonator and compressor. The setup is shown schematically in figure 7-9 and summarized as follows. In a double pass configuration, the stretcher (described in chapter 6.1) introduces a group delay dispersion of $1.19 \cdot 10^{8} \mathrm{fs}^{2}$ to the seed pulses and $70 \%$ throughput. The calculated corresponding Fourier-limited pulse duration of the $4 \mathrm{~nm}$ broad seed spectrum supported by the stretcher is $773 \mathrm{fs}$ (FWHM). After stretching to $430 \mathrm{ps}$, the double-stage fiber amplifier from the Friedrich Schiller University Jena increases the total seed energy to $\sim 0.7 \mathrm{~nJ}$. After this preamplification stage, a rubidium titanyl phosphate (RTP) Pockels cell is used to slice pulses from the $\sim 70 \mathrm{MHz}$ oscillator pulse train at a frequency between $1 \mathrm{kHz}$ and $20 \mathrm{kHz}$. An isolator protects the RTP PC against feedback from the amplifier. The BBO PC described in chapter 4.3 switches the selected pulses in and out of the amplifier resonator. The current beam diameter in the PC is $2.8 \mathrm{~mm}$ leading to a calculated B-integral of 1.67 for pulse energies of $\sim 30 \mathrm{~mJ}$. The $\sim 1 / 10 \mathrm{~mm}$ thin 0.1 degree wedged Yb:YAG disk mounted on a diamond heat sink is diode pumped with up to $500 \mathrm{~W}$ at $940 \mathrm{~nm}$ in a region smaller than $3 \mathrm{~mm}$ in diameter. Following amplification, recompression is performed using two transmission gratings with 1400 lines/mm arranged in a double pass Littrow-configuration (GDD $=-1.19 \cdot 10^{8}$ $\mathrm{fs}^{2}$ ), described in chapter 6.1. The cavity design shown in figure 6-16 was slightly changed for higher pump power (smaller radius of curvature of the disk) to obtain stable amplification for pump powers as high as $400 \mathrm{~W}$ and is shown in figure 7-10 together with the exact used components and distances are listed in table 7-1. 

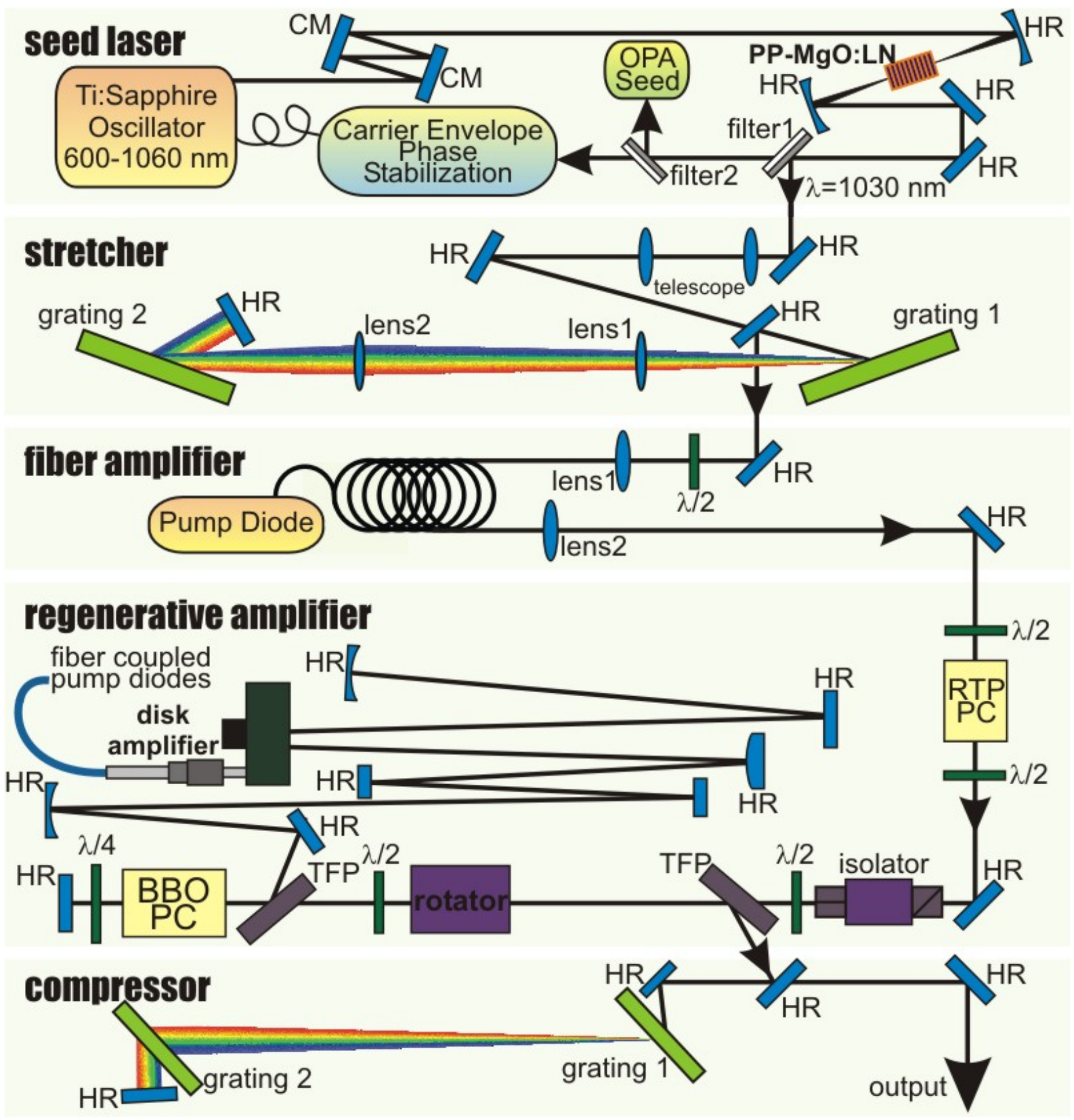

Fig. 7-9 Schematical regenerative amplifier setup with fiber preamplifier. HR, high reflecting mirror; CM, chirped mirror; PC, Pockels cell; TFP, thin-film polarizer; $\lambda / 2$, half-wave plate; $\lambda / 4$, quarter-wave plate.

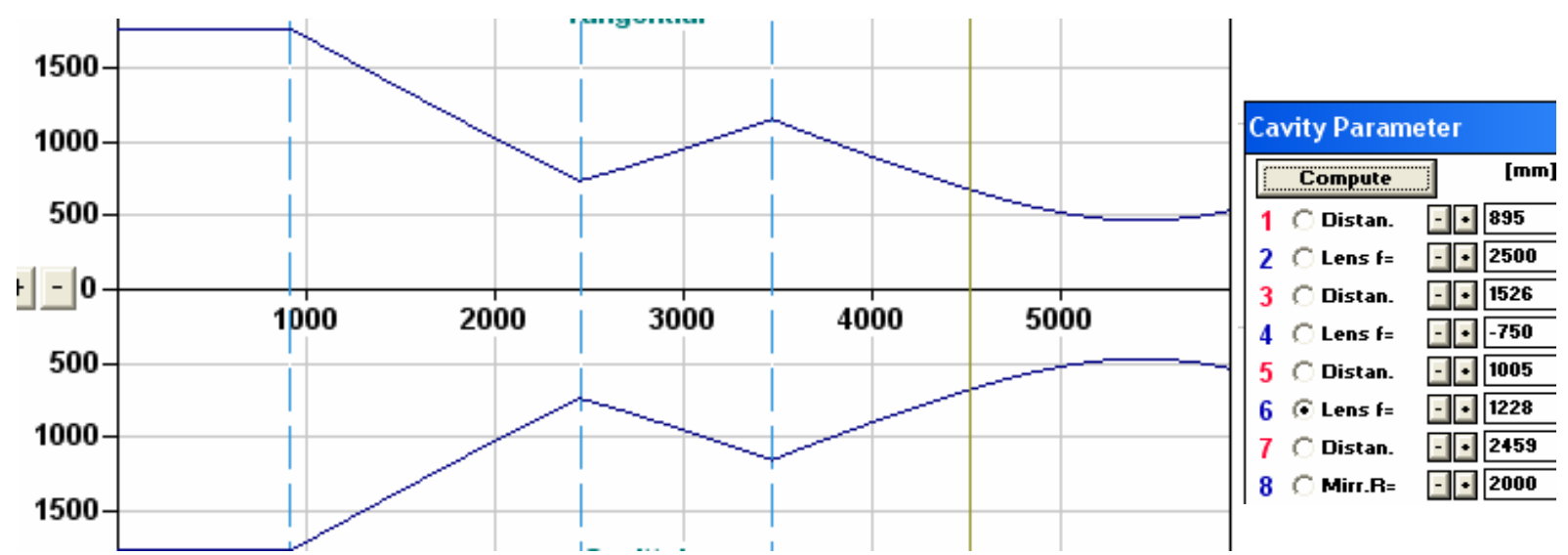

Fig. 7-10 Cavity design of the Yb:YAG disk based regenerative amplifier using a single pass through the disk. Beam diameter on the disk $\sim 2.45 \mathrm{~mm}$ and $\mathrm{PC} \sim 3.6 \mathrm{~mm}$. 


\begin{tabular}{c|c}
\hline mirror / distance / disk & radius of curvature / distance in $\mathrm{mm}$ \\
\hline end mirror & flat \\
distance & 895 \\
mirror concave & -2500 \\
distance & 1526 \\
mirror convex & +750 \\
distance & 1005 \\
disk concave & -1228 \\
distance & 2459 \\
end mirror concave & -2000 \\
\hline
\end{tabular}

Tab. 7-1 Distances and used mirrors in the designed cavity displayed in figure 7-10 listed from the left to the right side.

The continuous wave performance of the regenerative amplifier cavity design shown in figure 7-10 is depicted in figure 7-11. The $\mathrm{cw}$ output power between $55 \mathrm{~W}$ and $59 \mathrm{~W}$ at a pump power of $200 \mathrm{~W}$ was used as a reference value in case optical damage required a new cavity alignment. In case of lower $\mathrm{cw}$ output power than the reference value at $200 \mathrm{~W}$ of pump power one can consider bad alignment or an alignment over a dirty or damaged spot on one of the optics inside the amplifier.

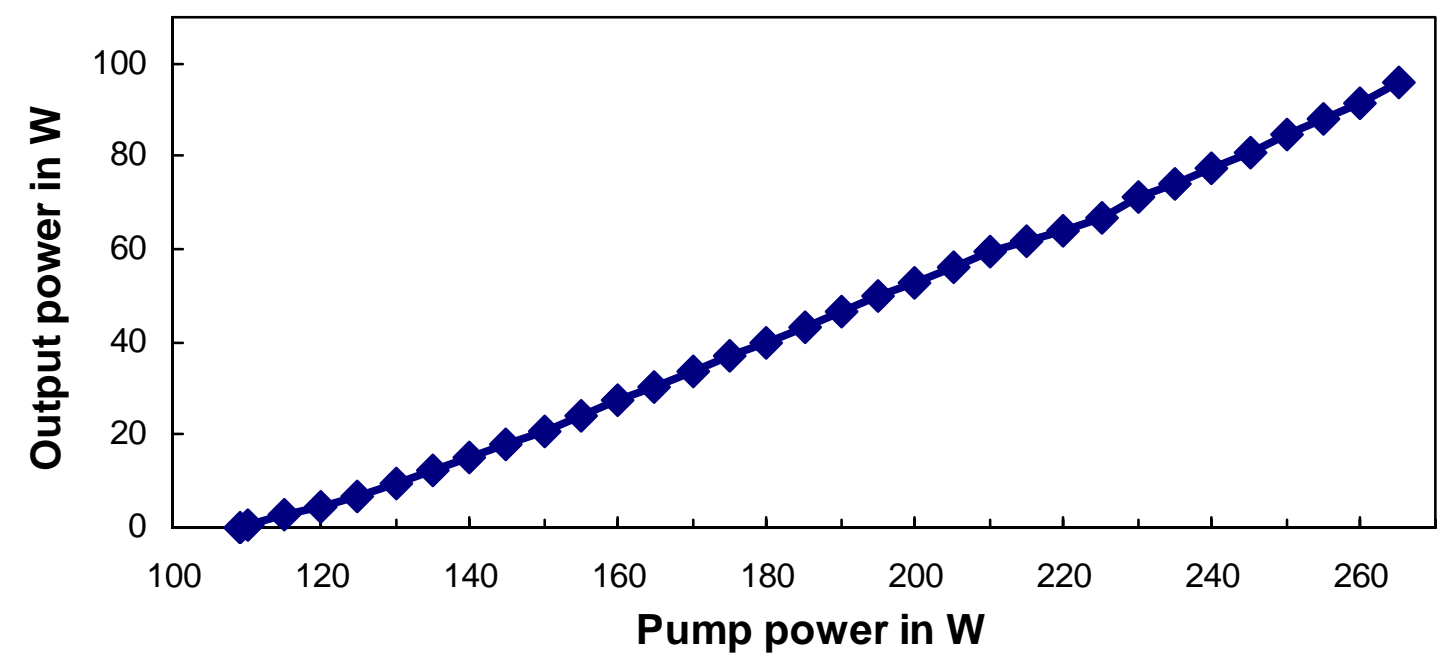

Fig. 7-11 Continuous wave performance of the amplifier cavity in dependence of the pump power.

\subsection{Results using the long stretcher}

To analyze the pulse build-up and the chaotic behavior of the system presented in figure 7-9, the pulse energy was recorded by varying the pump power (PP) in steps of $2 \mathrm{~W}$ between 100 and $300 \mathrm{~W}$ at a constant amplification time of $5.82 \mu$ s corresponding to 150 round trips (RT) in the regenerative resonator at a repetition rate of $6 \mathrm{kHz}$. For each PP setting, a train of 200 amplification cycles was traced by a fast photodiode with a rise time $<175 \mathrm{ps}$ and recorded with an oscilloscope. The pulse energy of each of the amplified 200 pulses in the measured sequence from the energy calibrated photo diode were determined and plotted versus the corresponding pump power in a so called bifurcation diagram. 
Figure 7-12 shows exemplary 20 recorded sequences of the out coupled pulses traced with the photo diode at a pump power of $136 \mathrm{~W}$. At this low pump power all pulses are amplified to similar energies $\sim 0.35 \mathrm{~mJ}$ at the full repetition rate of $6 \mathrm{kHz}$ and at this point no bifurcation appeared. Each sequence was recorded for $20 \mathrm{~ns}$ at a sampling rate of 1000 points.

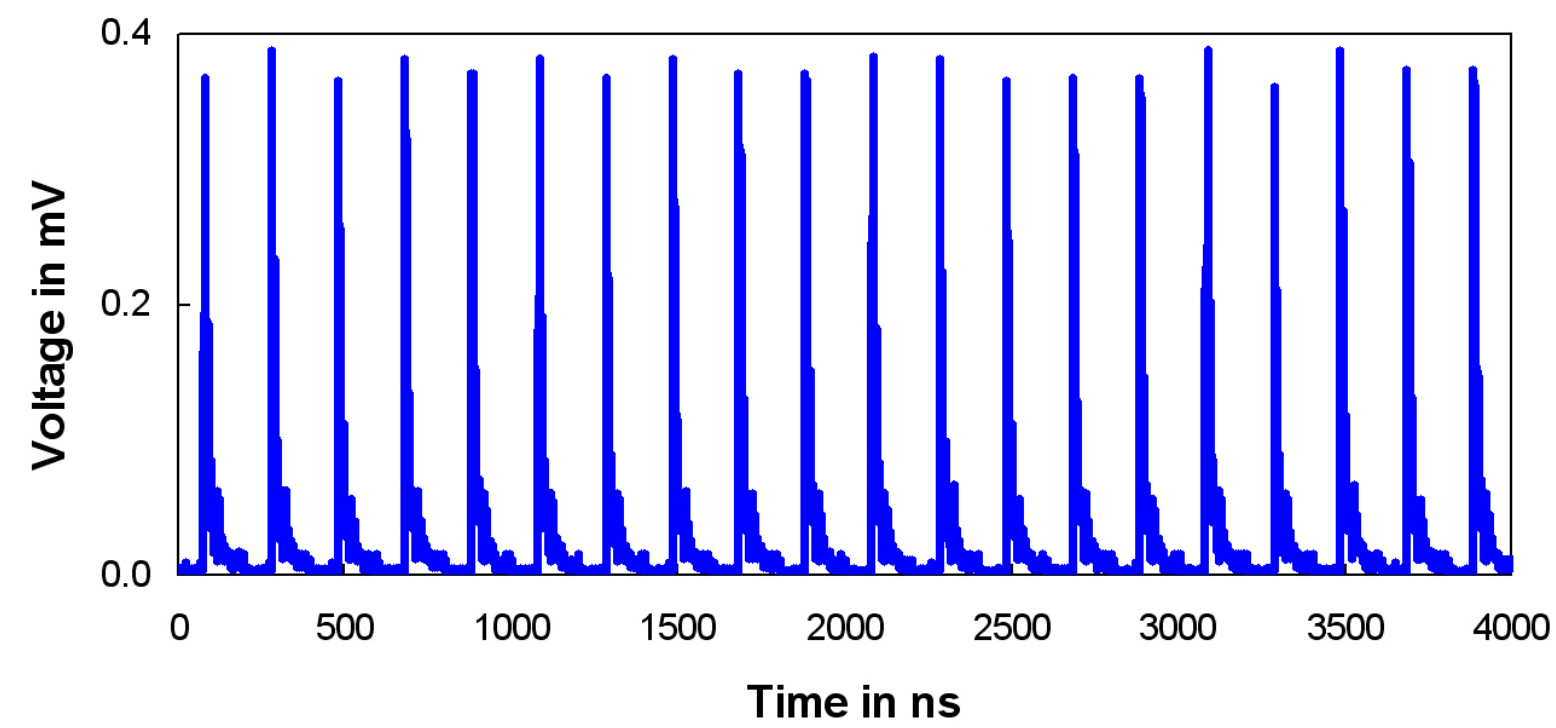

Fig. 7-12 Recorded photodiode signal for 20 sequences of the out coupled pulses at a pump power of 136 W. At this low pump power all pulses are amplified to similar energies $\sim 0.35 \mathrm{~mJ}$ at the full repetition rate of $6 \mathrm{kHz}$. Each sequence was recorded $20 \mathrm{~ns}$ long with a sampling rate of 1000 points.

Figure 7-13 shows 200 measured pulse sequences at $140 \mathrm{~W}$ of pump power overlapped in one diagram. Obvious the broad range of different peak heights indicating chaotic pulse build-up towards higher pump powers.

Figure 7-14 shows 20 sequences out of the chaotic pulse build-up at a pump power of $180 \mathrm{~W}$. Each pulse is coupled out after the same amount of round trips inside the regenerative amplifier resonator with different pulse energy. The pulse energy varies between a maximum of $25 \mathrm{~mJ}$ and a minimum of $0.3 \mathrm{~mJ}$.

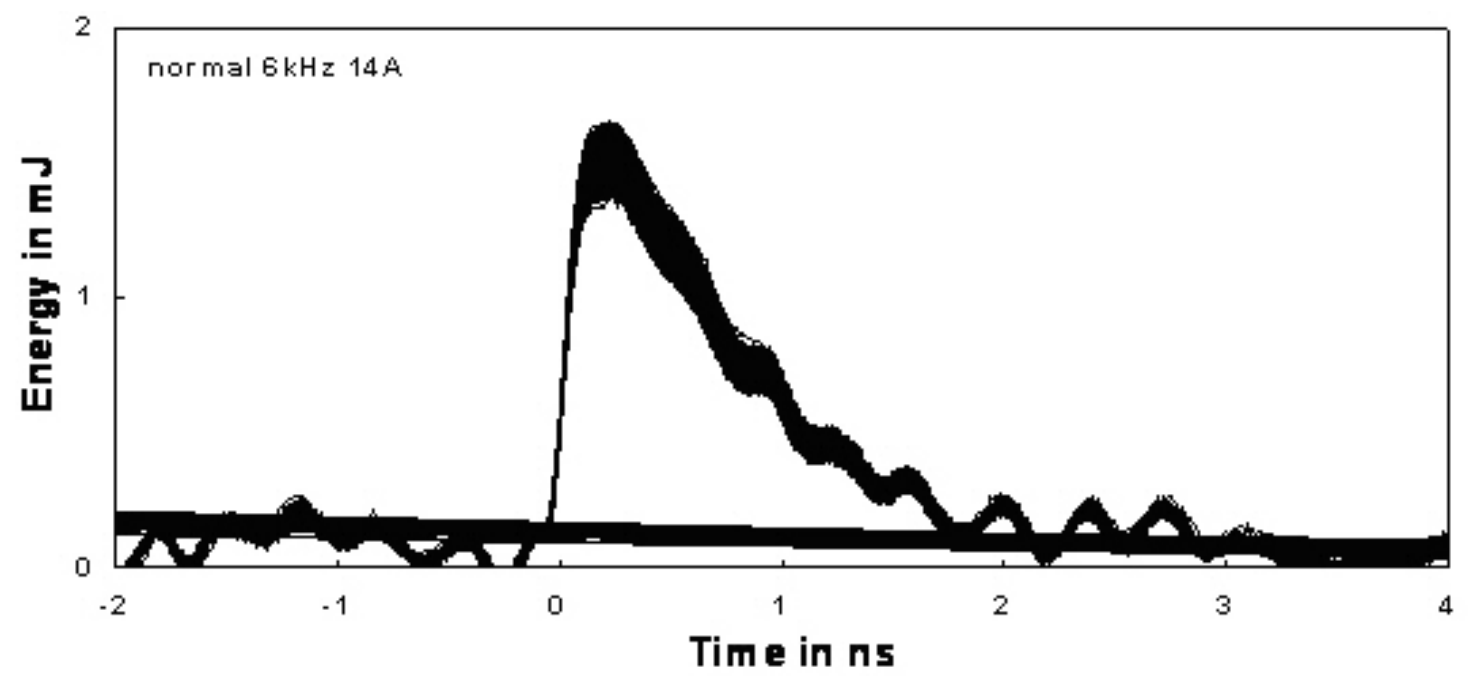

Fig. 7-13 200 measured pulse sequences at $140 \mathrm{~W}$ pump power overlapped in one diagram. The smeared out peaks indicate chaotic pulse build up towards higher pump powers. 


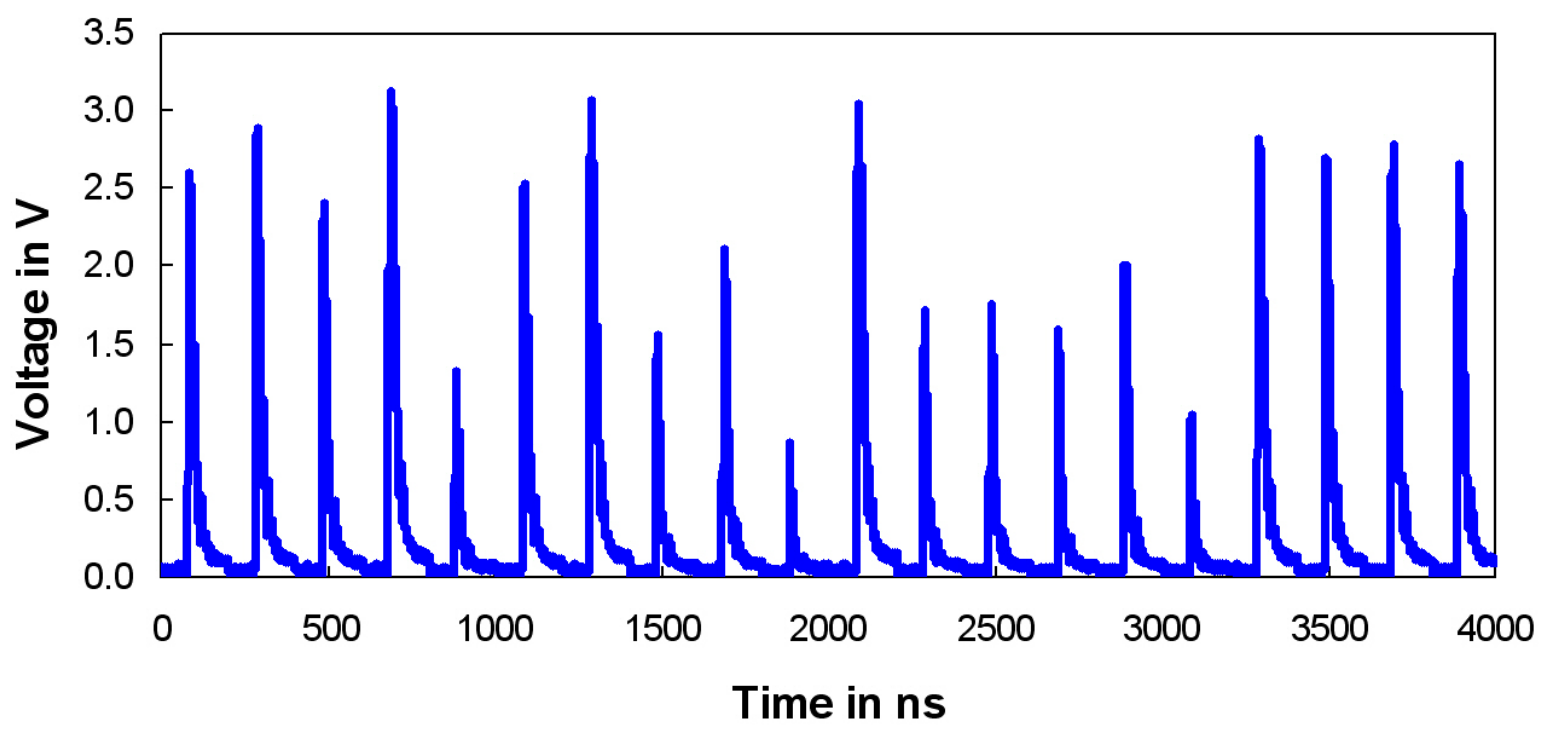

Fig. 7-14 20 recorded sequences out of the chaotic pulse build up at a pump power of $180 \mathrm{~W}$. The outcoupled energy varies between $25 \mathrm{~mJ}$ and $0.3 \mathrm{~mJ}$. Each sequence was recorded $20 \mathrm{~ns}$ long with a sampling rate of 1000 points.

Figure 7-15 shows 200 measured pulse sequences at $280 \mathrm{~W}$ of pump power overlapped in one diagram. The pulse build-up is completely chaotic and each pulse leaves the amplifier with a different energy. Figure 7-16 shows the corresponding energy distribution in a bifurcation diagram recorded by varying the pump power in steps of $2 \mathrm{~W}$ between 100 and $300 \mathrm{~W}$ at a constant amplification time of $5.82 \mu \mathrm{s}$ corresponding to 150 round trips in the regenerative amplifier resonator. For each pump power setting, a train of 200 amplification cycles was traced by a fast photodiode with a rise time $<175 \mathrm{ps}$ and recorded with an oscilloscope. The pulse energy of each of the amplified 200 pulses in the measured sequence from the energy calibrated photo diode were determined and plotted versus the corresponding pump power. To record the bifurcation diagram via the entire pump power range a peak finding software based on Matlab was used to detect the maxima of the 200 recorded sequences (figure 7-17) at each set value of pump power. The resulting bifurcation diagram bases on 20000 data points, where each data point corresponds to a measured single pulse energy.

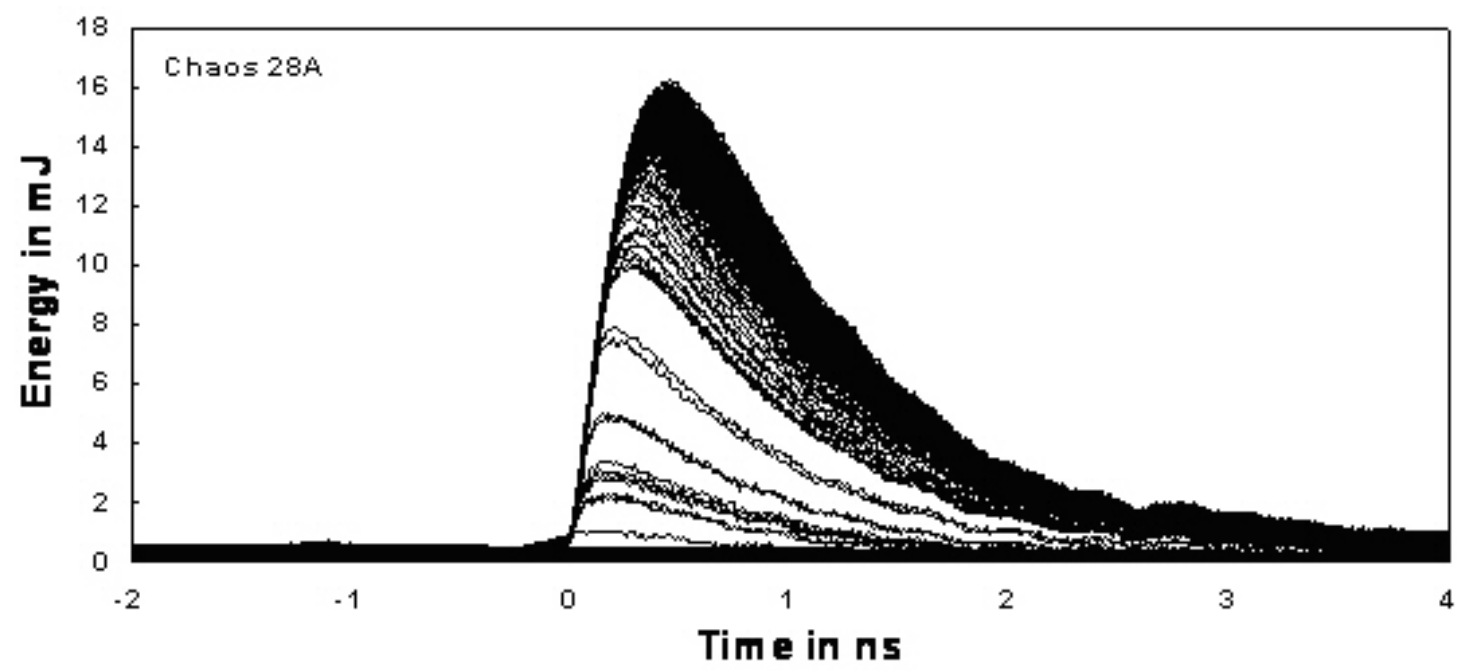

Fig. 7-15 200 measured pulse sequences at $280 \mathrm{~W}$ pump power overlapped in one diagram. The pulse build up is completely chaotic and each pulse leaves the amplifier with a different energy. 


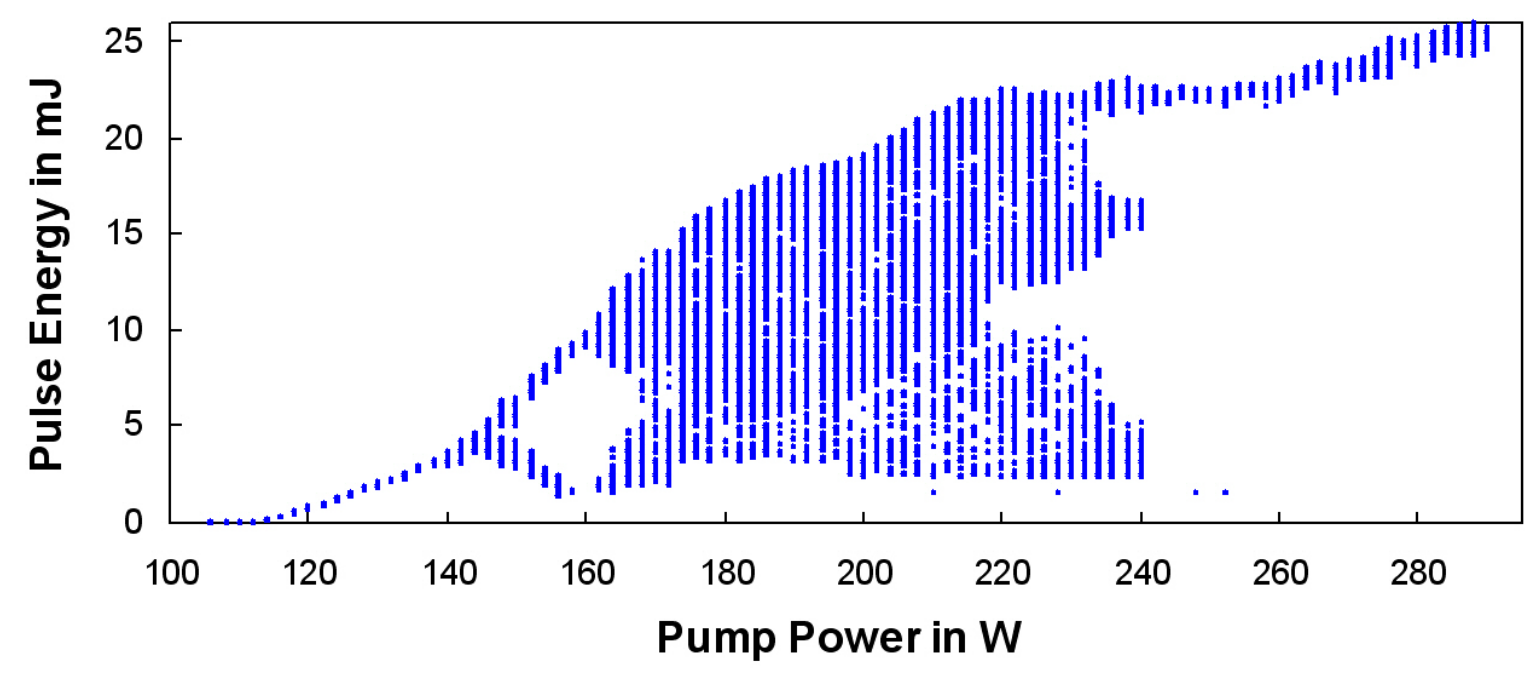

Fig. 7-16 Measured bifurcation diagram for 150 round trips in dependence of the pump power at a repetition rate of $6 \mathrm{kHz}$.
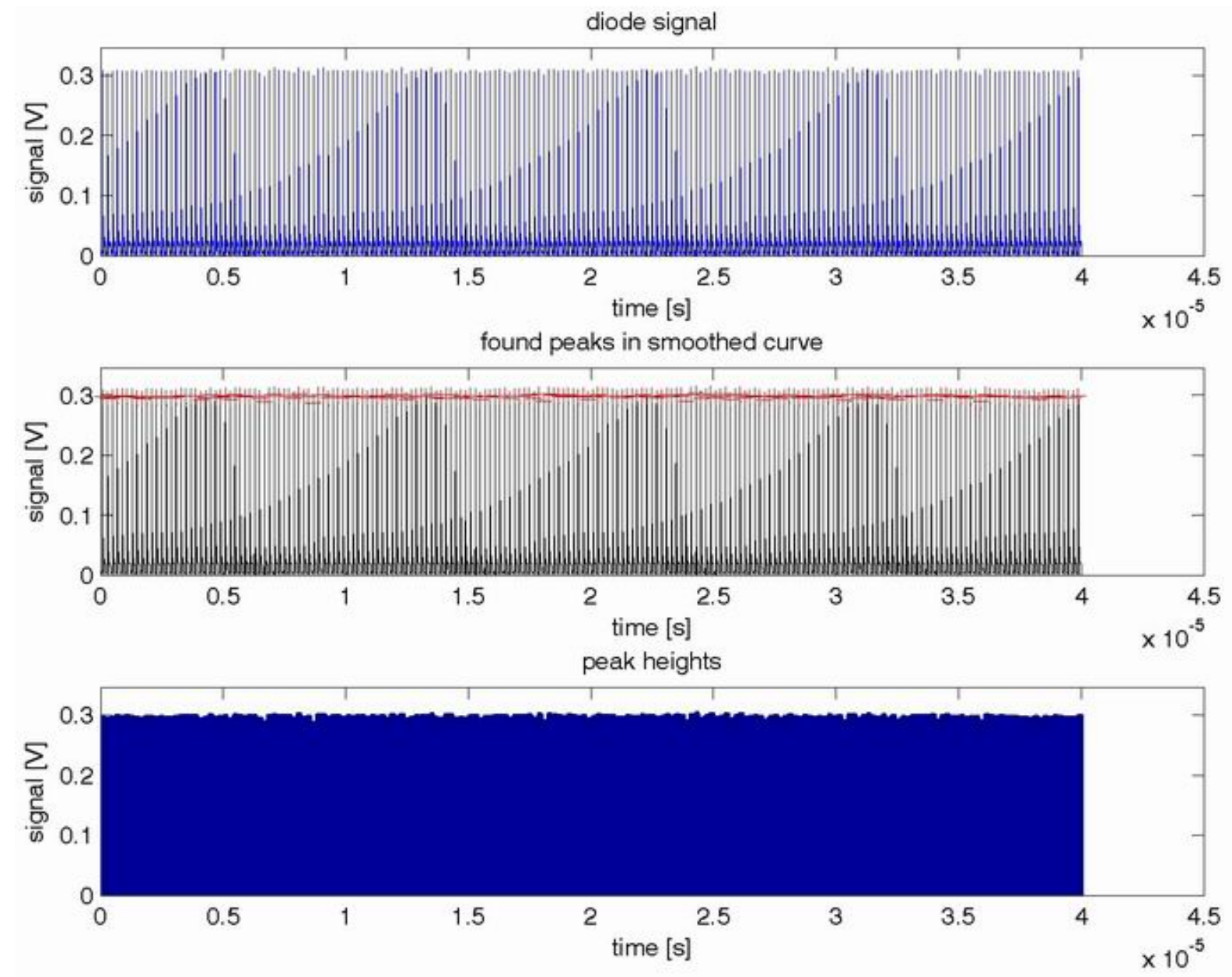

Fig. 7-17 Output scheme of the peak finding software.

For pump powers below $140 \mathrm{~W}$ normal amplification at the full repetition rate of $6 \mathrm{kHz}$ applied to the PC can be observed. Above $140 \mathrm{~W}$ the first bifurcation occurs, leading to completely chaotic pulse energies above $170 \mathrm{~W}$. A stable regime of period doubling commences for pump powers higher than $240 \mathrm{~W}$ resulting in a repetition rate of $3 \mathrm{kHz}$. Due to low thermal lensing in the disk, the amplifier resonator was stable over the full range of pump power. 
In the regime of period doubling the contrast between the two adjacent pulses coupled out by the $\mathrm{PC}$ at a repetition rate of $6 \mathrm{kHz}$ is higher than a factor of 500 and as mentioned before from an application standpoint can be safely neglected, as the OPA gain scales as the exponential of the square root of the pump intensity [10]. The typical maximum pulse energy of $25 \mathrm{~mJ}$ was measured at $284 \mathrm{~W}$ of pump power with an average output power of $75 \mathrm{~W}$ after the compression. The period doubling regime provides highly stable output pulse energies due to saturation of the gain media allowing for every second pulse to extract the entire stored energy from the pumped area of the disk. Taking the compression losses into account, the total optical efficiency was calculated to be $26 \%$.

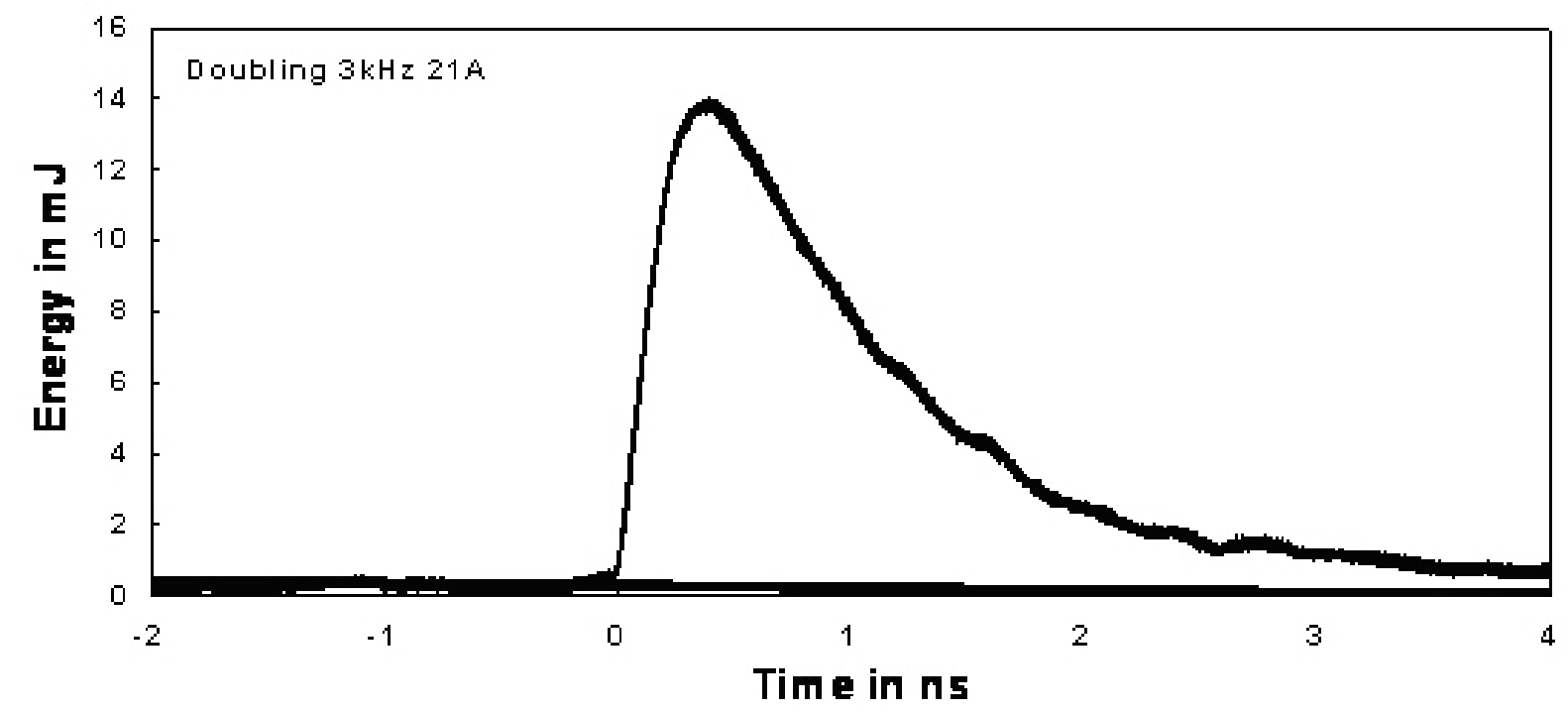

Fig. 7-18 200 overlapped measured sequences at $210 \mathrm{~W}$ pump power in a stable period doubling regime.

Figure 7-18 shows 200 overlapped sequences for the case of stable period doubling at $3 \mathrm{kHz}$ and visualizes the small energy fluctuation of $<0.5 \%$ and the total absence of any other pulses containing less energy.

In case of period doubling the regenerative amplifier needs to be saturated in average power. Stable single energy period doubling derives only when the energy stored in the laser gain media is completely extracted during the first amplification cycle and is not sufficiently recovered until the following pulse is switched into the resonator amplifier. Figure 7-19 shows the pulse build up (green curve) in the regenerative amplifier together with the out coupled pulse (yellow) for one amplification cycle in the period doubling regime for a pump power of $212 \mathrm{~W}$, a resulting repetition rate of $3 \mathrm{kHz}$ leading to $13 \mathrm{~mJ}$ pulse energy after the compression with a root-mean-square (RMS) fluctuation in pulse energy of $<0.52 \%$ at $5.8 \mu$ s amplification time corresponding to $\sim 150$ round trips inside the amplifier cavity.

Operating within an intermittent stable regime in the deterministic chaos of the continuously pumped regenerative amplifier shown in figure 7-16, an average output power of $>75 \mathrm{~W}$ at a repetition rate of $3 \mathrm{kHz}$ with pulse energies exceeding $25 \mathrm{~mJ}$, a pulse-to-pulse stability of $<0.7 \%$ (RMS), a pulse duration of $1.6 \mathrm{ps}$ and a bandwidth of $1 \mathrm{~nm}$ (FWHM) at a center wavelength of $1030.2 \mathrm{~nm}$ were measured. Figure 7-20 shows the measured autocorrelation trace and the optical spectrum of the laser output at $25 \mathrm{~mJ}$. The resulting time bandwidth product of 0.46 is within $5 \%$ of the transform limit of 0.441 for Gaussian pulses. The meas- 
ured spectrum for the output pulses amplified to energies of $25.0 \mathrm{~mJ}$ pulse energy at a repetition rate of $3.0 \mathrm{kHz}$ in the period doubling regime is shown in figure 7-21.

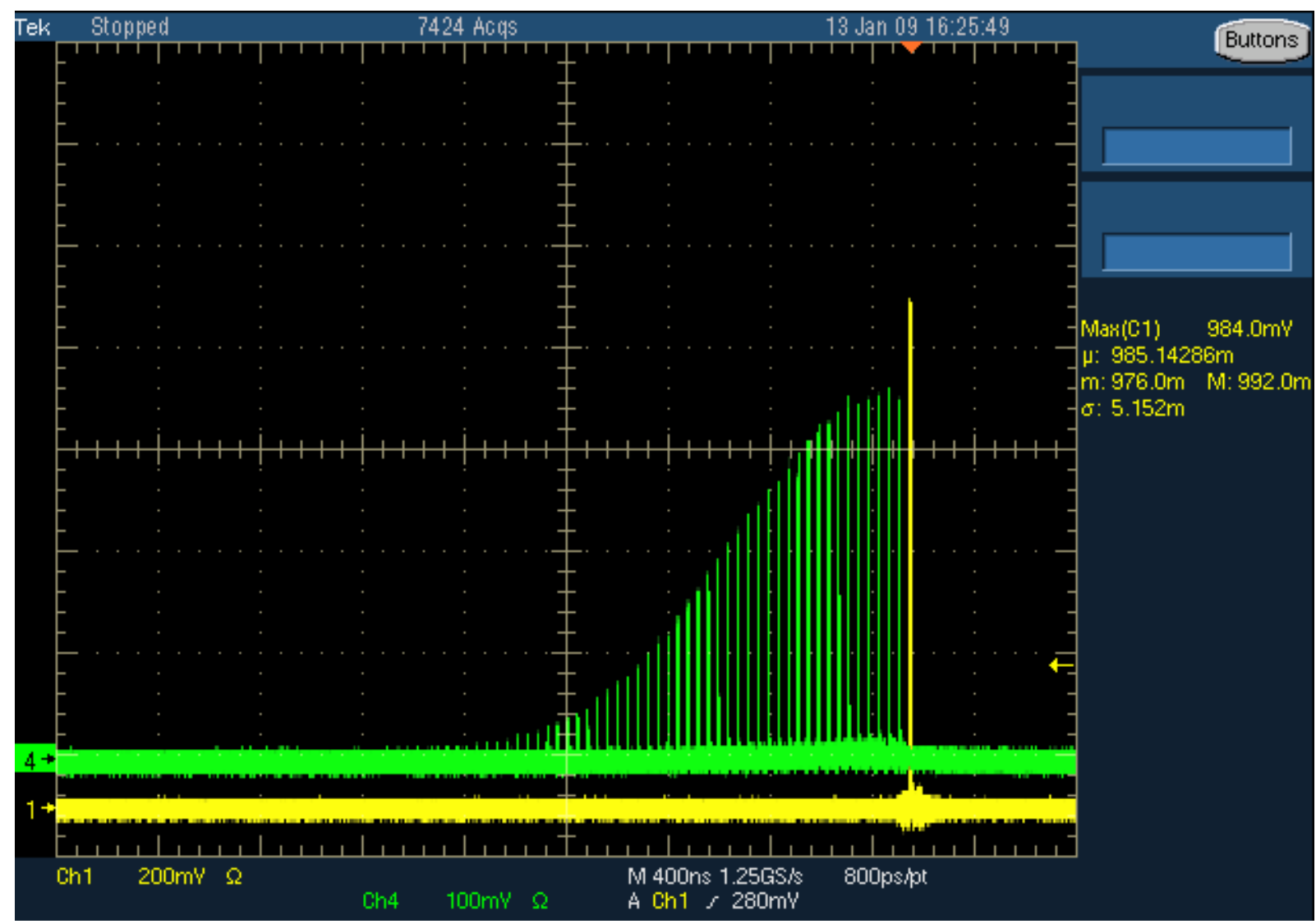

Fig. 7-19 Pulse build up (green curve) in the regenerative amplifier together with the out coupled pulse (yellow) in the period doubling regime (pump power $212 \mathrm{~W}$, resulting repetition rate of $3 \mathrm{kHz}$, $13 \mathrm{~mJ}$ pulse energy after the compression, RMS fluctuation $<0.52 \%, 5.8 \mu \mathrm{s}$ amplification time $=150$ round trips inside the amplifier cavity).

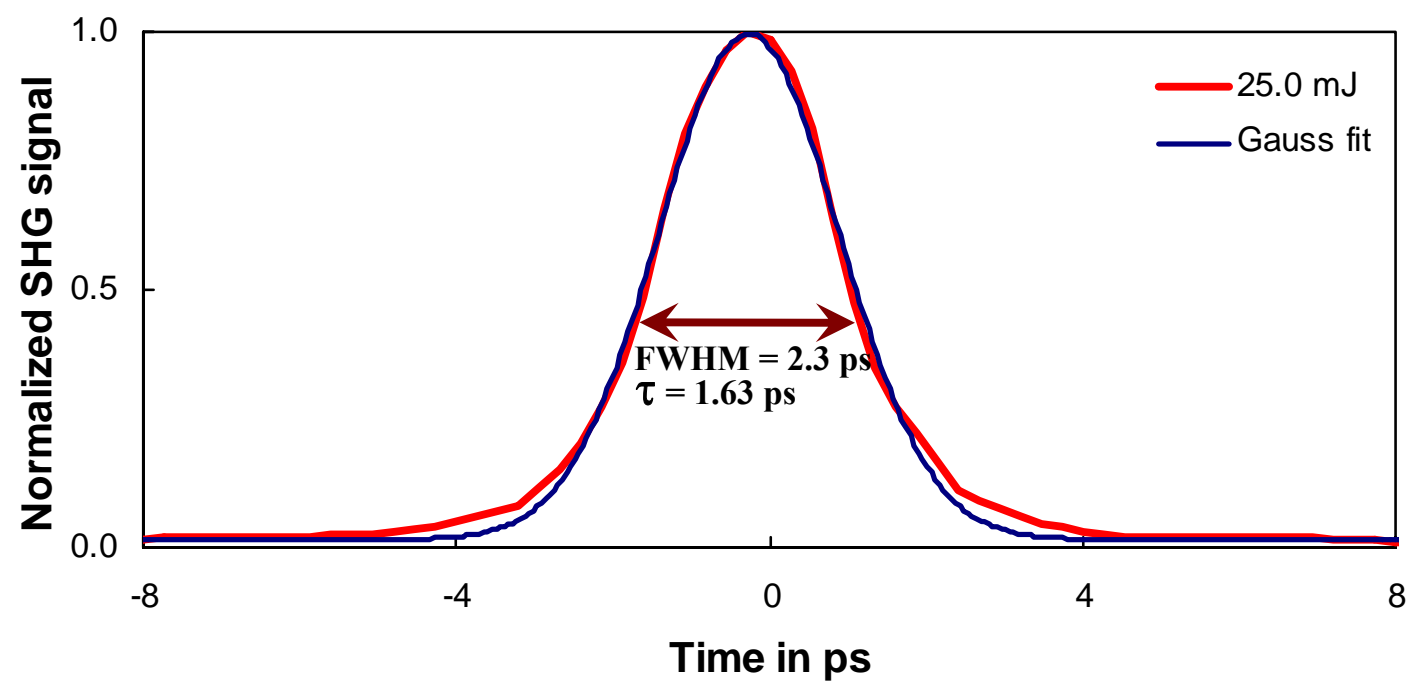

Fig. 7-20 Measured SHG autocorrelation of the compressed output pulses at $25.0 \mathrm{~mJ}$ pulse energy at a repetition rate of $3.0 \mathrm{kHz}$ in the period doubling regime $(280 \mathrm{~W}$ pump power, 150 round trips, RMS fluctuation $<1.0 \%$ ).

Gain narrowing reduces the bandwidth to $1 \mathrm{~nm}$ and steepens the pulse duration inside the amplifier cavity to 200 ps before compression. Taking the compression losses of $77 \%$ into ac- 
count, the total optical efficiency was calculated to be $26 \%$. In addition to pulse stability of $1 \%$ at $25.0 \mathrm{~mJ}$ and $3.0 \mathrm{kHz}$, the disk-amplifier also exhibits good spatial beam qualities necessary for homogeneous parametric amplification.

Beyond a superior stability of pulse energy, the disk-amplifier also exhibits an excellent beam quality, which is also critical for parametric amplification. The amplifier cavity supports only the $\mathrm{TEM}_{00}$ fundamental laser mode, resulting in a near diffraction-limited beam with $\mathrm{M}^{2}=1.03$ measured at the maximum pulse energy with a commercial camera-based system according to ISO Standard 11146. The beam profile is shown in figure 7-22 followed by the measured beam caustic depicted in figure 7-23.

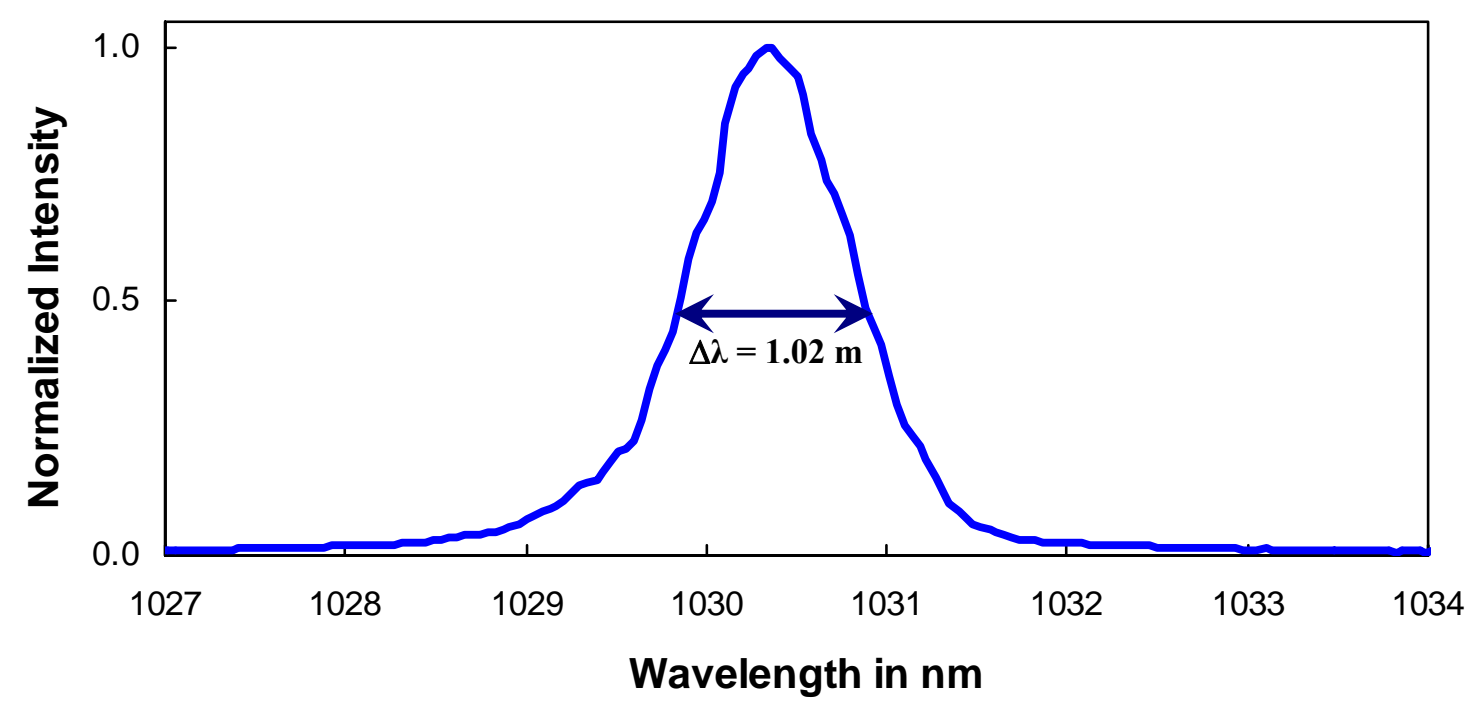

Fig. 7-21 Measured spectrum for output pulse energies of $25.0 \mathrm{~mJ}$ at a repetition rate of $3.0 \mathrm{kHz}$ in the period doubling regime (280 W pump power, 150 round trips, $R M S$ fluctuation $<1.0 \%$ ).
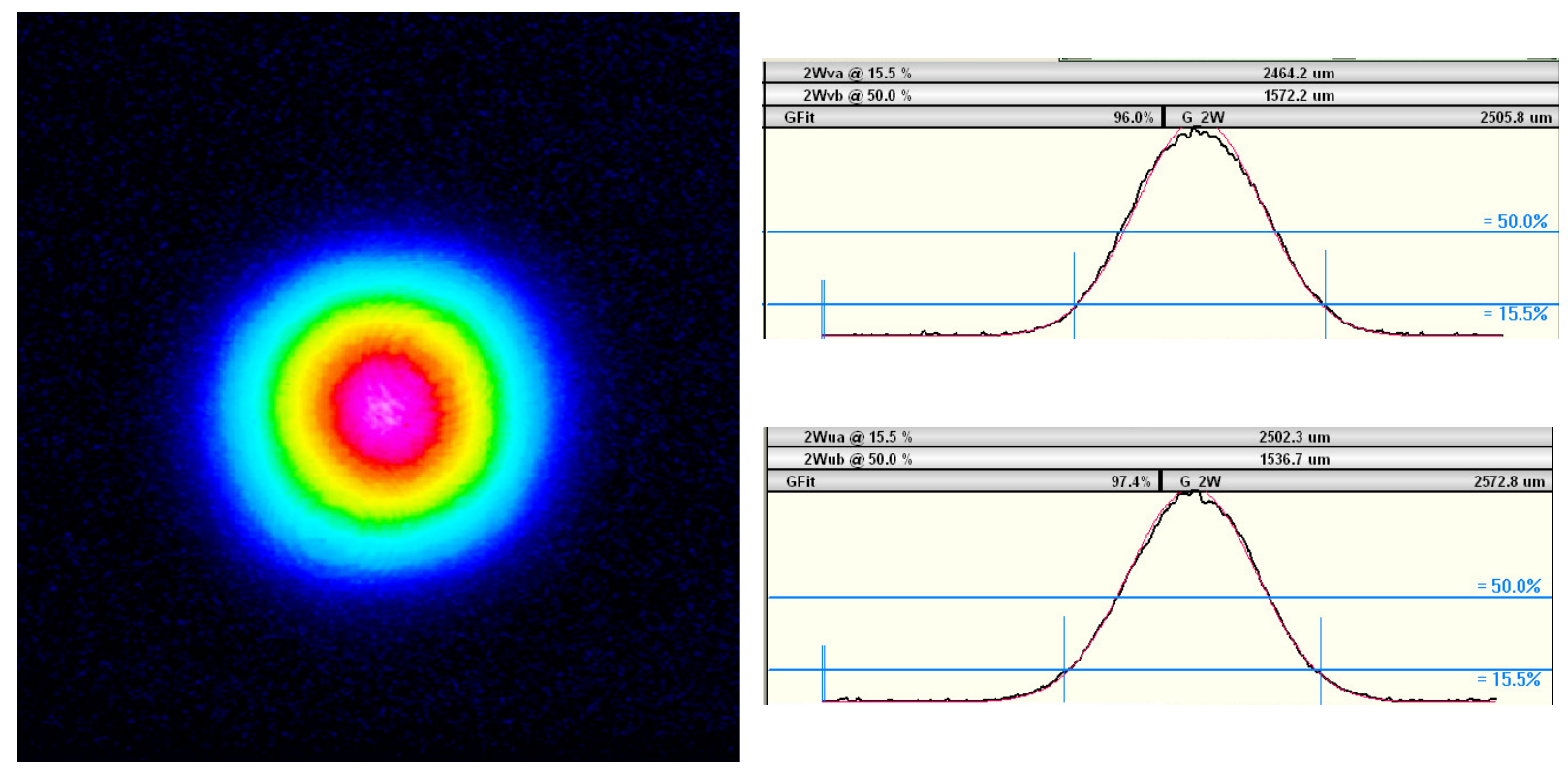

Fig. 7-22 Beam profile after out coupling of the regenerative amplifier $(25.0 \mathrm{~mJ}, 3.0 \mathrm{kHz}, 280 \mathrm{~W}$ pump power, 150 round trips, RMS fluctuation $<1.0 \%$ ).

By varying parameters like the number of round trips and pump power, which affect the laser's bifurcation characteristics [104,110,111], the intermittent stable pulse regime of period 
doubling can be shifted to different single-energy ranges allowing output energies between 2 and $25 \mathrm{~mJ}$ at $3 \mathrm{kHz}$. For example, by using fewer round trips, the intermittent stable regime is followed by a further chaotic interval and stable output energy occurs between a lower and upper pump power limit for period doubling. The measured pump power dependent bifurcation diagram for 120 amplifier round trips in figure 7-24 shows an intermittent stable period doubling regime between $200 \mathrm{~W}$ and $240 \mathrm{~W}$ of pump power.

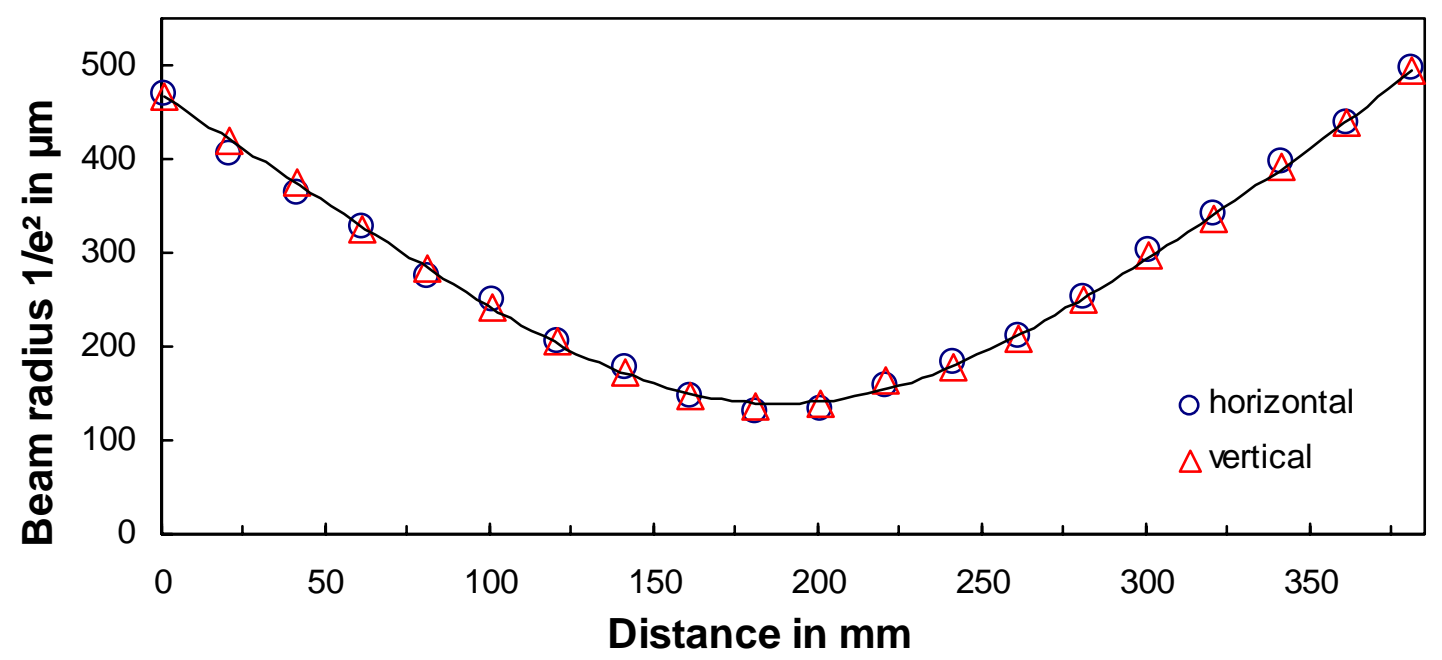

Fig. 7-23 Measured caustic according to ISO Standard 11146 for calculating the beam quality and the $\mathrm{M}^{2}$ factor.

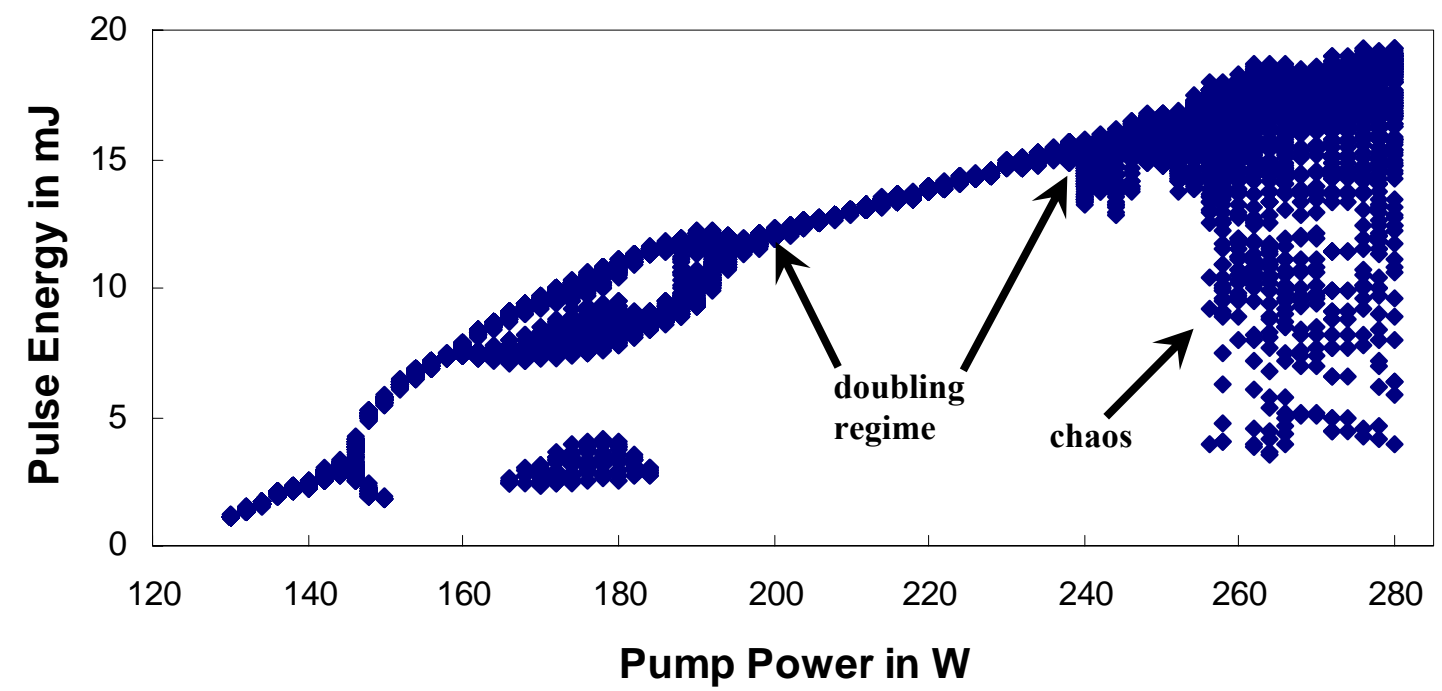

Fig. 7-24 Measured bifurcation diagram for 120 round trips for varying pump power.

Figure 7-25 shows the influence of the pump power on the lower and upper period doubling limits for different number of round trips varying between 85 and 230 corresponding to an amplification time between 3.3 and $9.0 \mu \mathrm{s}$. The higher the number of round trips the lower the period doubling energy. High out put energies require a lower number of round trips and higher pump power. Figure 7-26 shows the same measurement for the dependency of the lower and upper limits of period doubling but in a different illustration versus the number of round trips and confirms the cognizance that for higher output energies less round trips are required and vice versa. But it also shows the achievable pulse energy for period doubling at a 
given number of round trips. The less the number of round trips the broader the single energy regime of period doubling. Below 84 round trips, no chaotic pulse build up could be detected. A short range of pulse energy splitting is followed by a broad range of stable single energy period doubling between pump powers of 120 and $360 \mathrm{~W}$ delivering output energies between 5 and $26 \mathrm{~mJ}$.

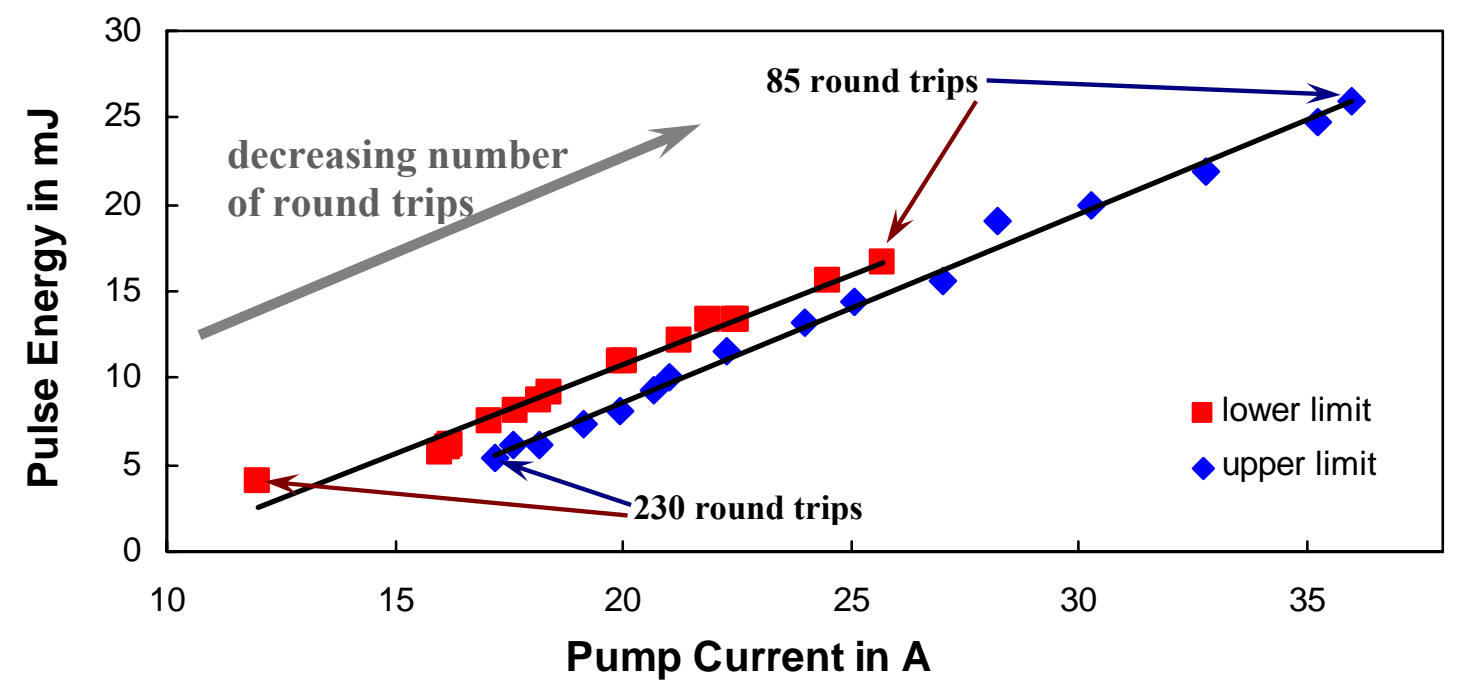

Fig. 7-25 Lower and upper period doubling limits versus the pump power for different number of round trips for a $\mathrm{PC}$ repetition rate of $6 \mathrm{kHz}$ leading to $3 \mathrm{kHz}$ periode doubled output pulses (between 85 and 230 round trips corresponding to 3.3 and $9.0 \mu$ s amplification time).

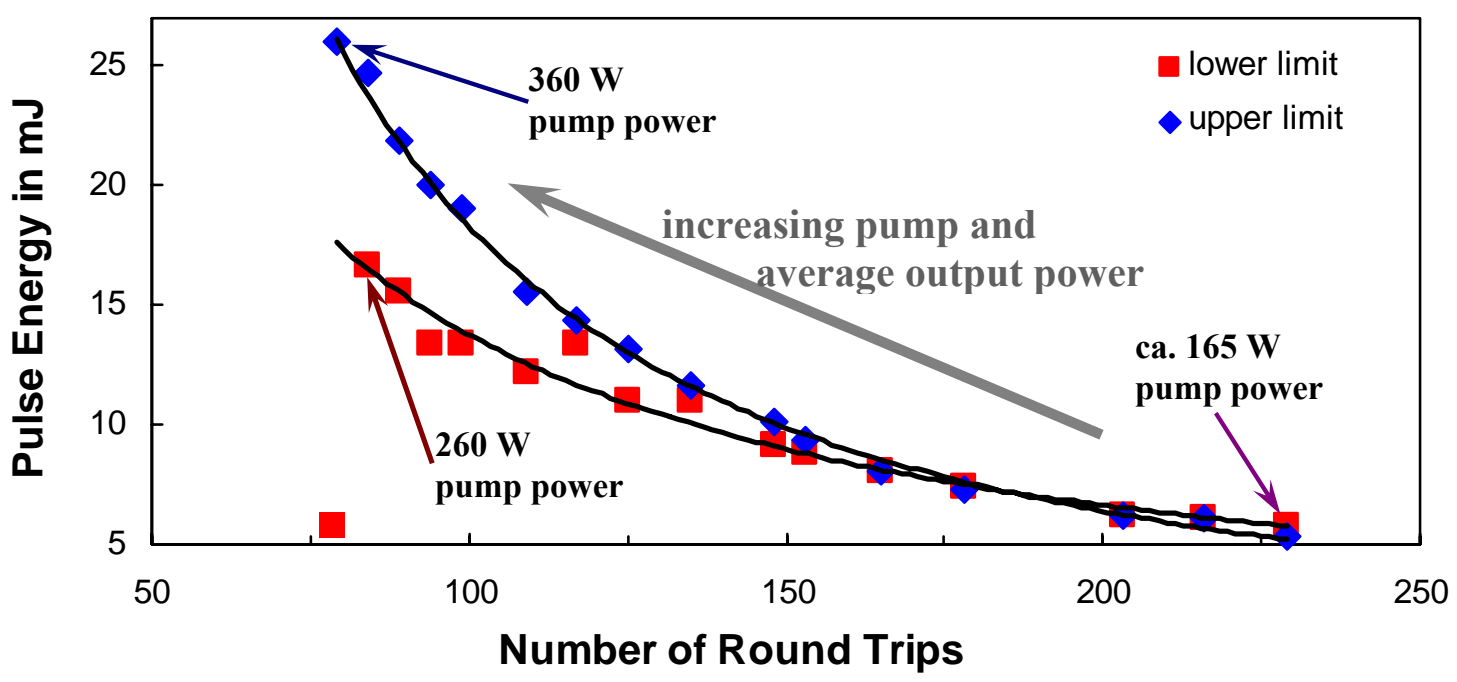

Fig. 7-26 Lower and upper period doubling limits versus the number of round trips for different pump powers at a PC repetition rate of $6 \mathrm{kHz}$ leading to $3 \mathrm{kHz}$ period doubled output pulses. (between 165 and $230 \mathrm{~W}$ pump power).

In conclusion, the regenerative chirped pulse amplifier based on a $\mathrm{Yb}: \mathrm{YAG}$ thin disk achieves at a repetition rate of $3.0 \mathrm{kHz}$ in a near diffraction-limited beam with $\mathrm{M}^{2}=1.03$ pulse energies of $25 \mathrm{~mJ}$ with a pulse duration of $1.6 \mathrm{ps}$ at a wavelength of $1030.2 \mathrm{~nm}$ and a pulse-to-pulse stability $<0.7 \%$ optical synchronized to a Ti:sapphire oscillator. The peak power of $>15 \mathrm{GW}$ exceeds all reported values for regenerative amplifier in the multi-kHz range. 


\section{Nonlinear compressor}

Spectral broadening of laser pulses by SPM in nonlinear fibers or gases followed by chirp compensation in suitable phase-dispersive elements is a well-established technique for pulse shortening, especially for the generation of few cycle pulses. In 1997 pulse durations below $5 \mathrm{fs}$ could be achieved by focusing $20 \mathrm{fs}$ long pulses of a Ti:sapphire laser into a krypton filled hollow fiber followed by a chirped mirror compressor [112]. Using a similar, improved system pulse durations below 4 fs could be demonstrated with sub-mJ energy in 2007 [7]. But also high average power lasers with longer pulse durations around 800 fs could be further compressed using a large-mode-area holey fiber to broaden the spectra followed by a prism based compressor resulting in shortened pulse durations of $33 \mathrm{fs}$ at $34 \mathrm{MHz}$ repetition rate and an average power of $18 \mathrm{~W}$ [113].

Broadening the spectra of higher energetic pulses via SPM bulk material like sapphire plates or gas cells can be used to avoid optical damage in hollow fibers or nonlinear fibers. To further reduce the pulse duration of the regenerative amplifier output a high gas pressure cell and an additional grating based compressor had been applied. The high gas pressure cell had been constructed originally for an ultrabroadband, coherent light source for waveform synthesis [114]. The $60 \mathrm{~cm}$ long gas cell has been designed to sustain high gas pressure, adjustable in the range of 0 to 80 bar enclosed between two $1 \mathrm{~mm}$ thin for $1030 \mathrm{~nm}$ anti-reflex coated fused-silica windows with a clear aperture of $8 \mathrm{~mm}$ and is shown in figure 8-1.

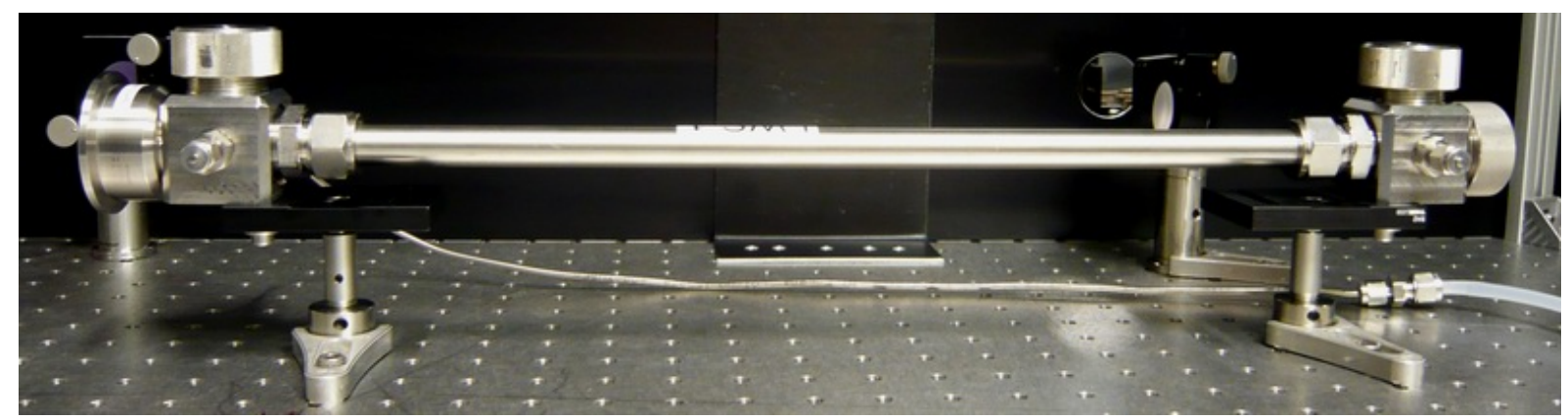

Fig. 8-1 High gas pressure cell for spectral broadening via self phase modulation of the laser beam.

To avoid spatial beam distortions due to plasma formation and self-channeling, the laser peak power in the gas cell must be smaller than the self-focusing value and below the multiphoton ionization threshold, which applies for the given pulse duration. In a preliminary selfchanneling test, the beam with $10 \mathrm{~mm}$ diameter, pulse energies of $10 \mathrm{~mJ}$ at a repetition rate of $3.0 \mathrm{kHz}$ was focused by a $1000 \mathrm{~mm}$ lens into the first evacuated cell which was than refilled and pressurized with noble gas until white light generation and plasma formation was detected.

Using this method, different gases were analyzed at varying pressures for maximum spectral broadening. In first experiments lower pulse energies of $10 \mathrm{~mJ}$ were chosen to avoid optical damage of the entrance window of the gas cell and the following optics like out-put window, lenses and gratings in case of self focusing and self channeling caused by too high gas pressures in the cell while searching for the optimum gas pressure. Using a second $1000 \mathrm{~m}$ focal 
lens the beam was recollimated and further compressed in a delay line introducing an additional GDD of $-1.3 \cdot 10^{5} \mathrm{fs}^{2}$. The compressor consisted of two transmission gratings with 1400 lines/mm separated by $6 \mathrm{~mm}$ in a similar set up introduced in chapter 6.1 and is shown in figure 6-6 with an overall transmission of $80 \%$ in a double pass. The entire setup for the nonlinear compression scheme is shown in figure $8-2$.

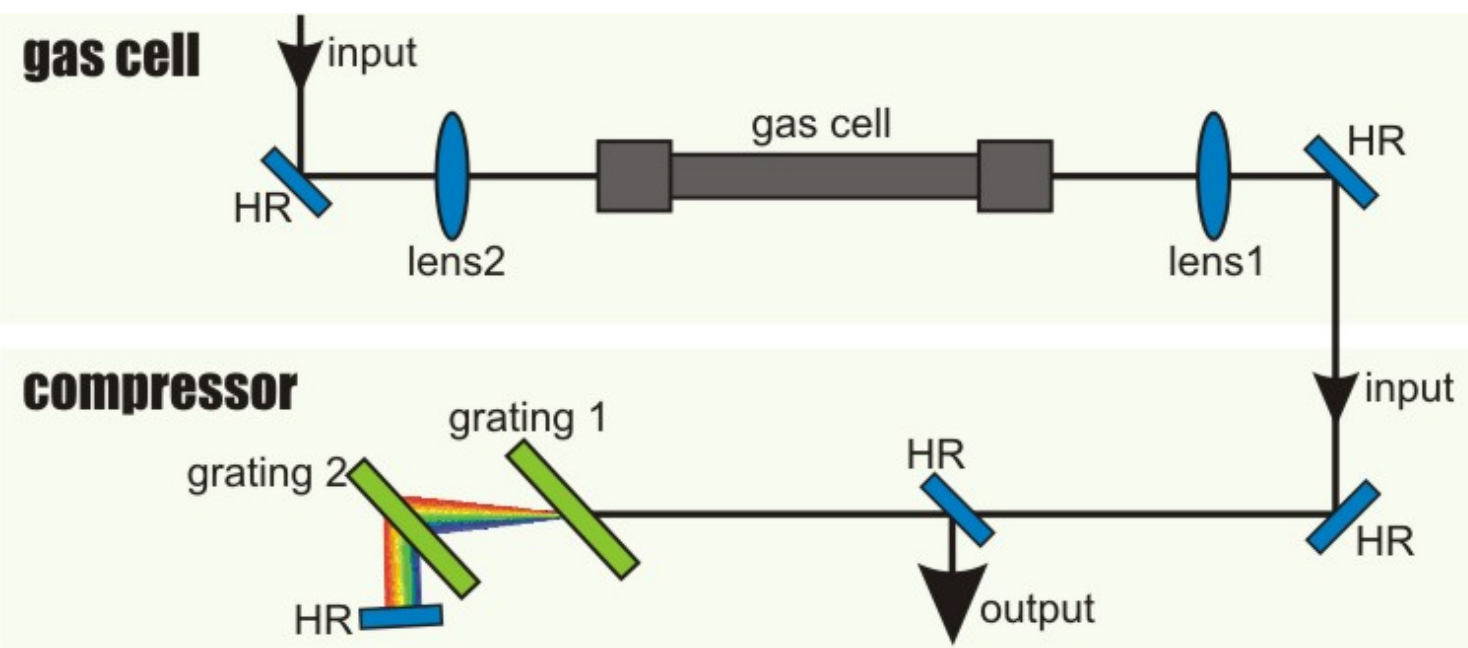

Fig. 8-2 Schematical setup of the nonlinear compressor using a gas cell for spectral broadening and a transmission grating based compressor.

In the preliminary setup, the maximum gas pressure was limited to 5.0 bar by the manometer used and the connected hoses. Spectral broadening was explored for Helium, Xenon, Krypton, Argon and Neon. Up to 5.0 bar, no significant spectral broadening was observed by using Helium and Neon. The spectra for Xenon, Krypton and Argon measured below the threshold of SF are shown in figure 8-3 in comparison to the measured spectrum by using the evacuated gas cell.

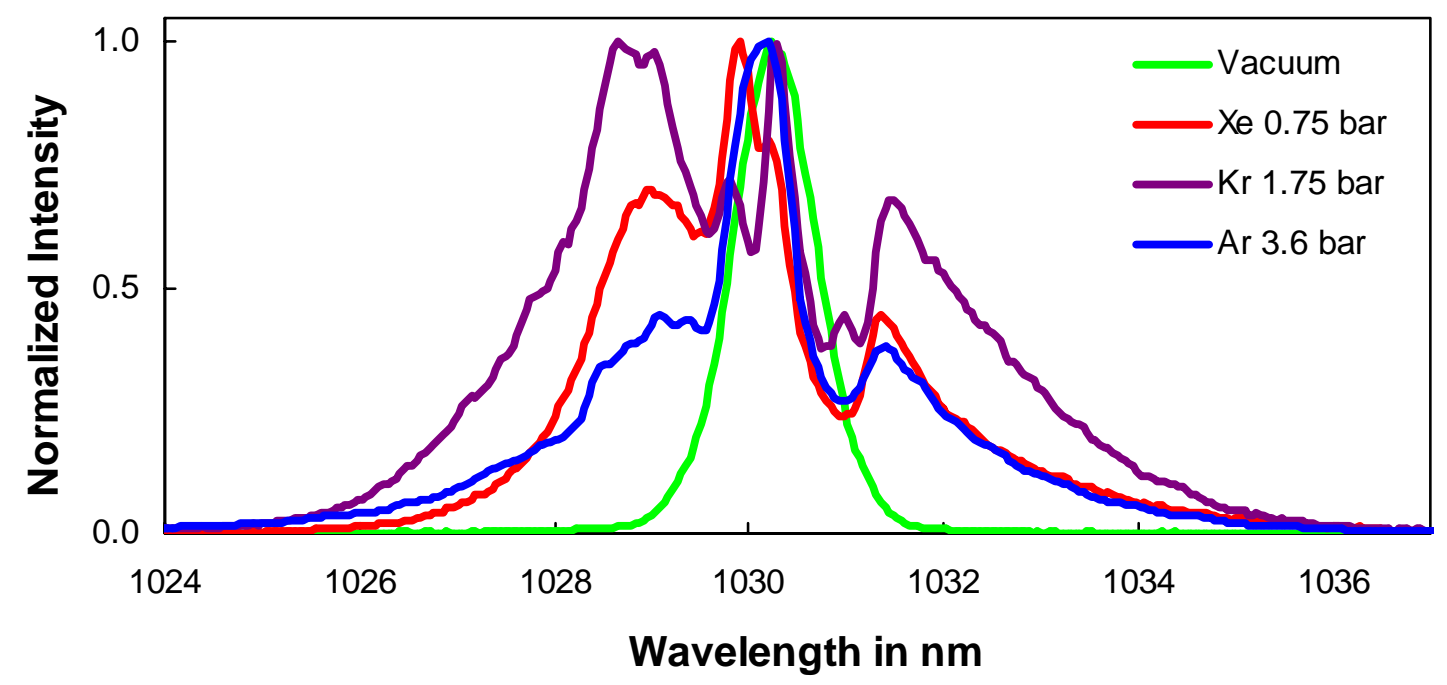

Fig. 8-3 Measured broadened spectra due to self phase modulation in different noble gases compared to the initial spectrum.

The gases Xenon and Krypton led already at low absolute pressures to significant spectral broadening. Unfortunately the threshold between SPM and self-channeling was very small in both gases and difficult to adjust precisely by using the pressure reducer of the gas bottle and 
valves to refill the evacuated gas cell. The most reliable result could be achieved in 3.6 bar of Argon. Argon was therefore used to perform the further compression although the observed broadening was less than for Xenon and Krypton.

After the recompression, a clean autocorrelation could be measured for a pressure at 2.8 bar of Argon. The pulse duration was measured to be $600 \mathrm{fs}$. At an increased pressure of 3.6 bar the pulse duration could be decreased to $450 \mathrm{fs}$ but unfortunately with side wings in the autocorrelation trace due to imperfect recompression.

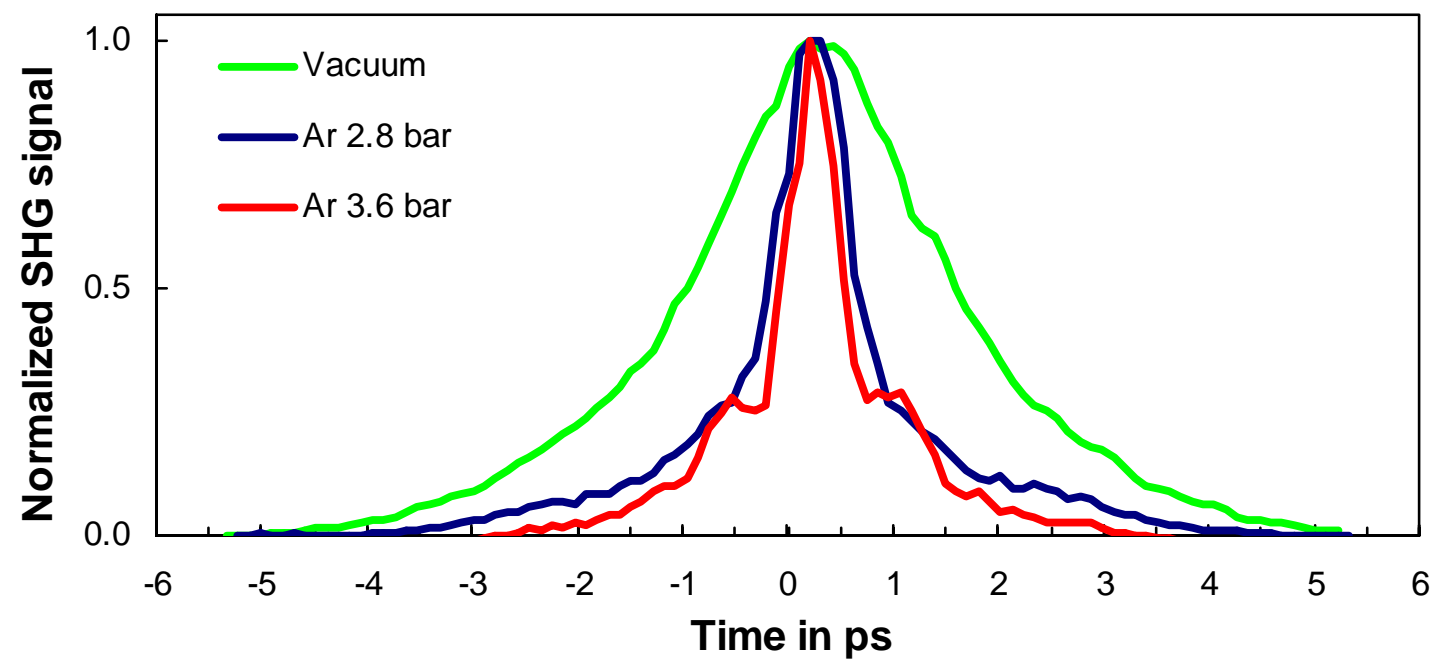

Fig. 8-4 Measured autocorrelation traces after the nonlinear compression scheme shown in figure 8-2.

The experiment can only be seen as preliminary and incomplete but nevertheless it could be demonstrated that significant pulse shortening is possible by using a nonlinear compression scheme. Other experiments with femtosecond lasers showed that gentle focusing conditions lead to an improved performance and a broader stable regime between SPM and selfchanneling. The use of a longer focusing lens and a longer gas cell would lead to a longer interaction length between the laser beam and the gas and could improve the mechanism of spectral broadening due to SPM. Larger entrance and output windows with an increased aperture would further allow to use the full pulse energy of disposal. The compressor using two transmission gratings was simple to align but for future experiments the distance between the two gratings needs to be adjustable via micrometer screws to perform better compression. 


\section{Multipass booster amplifier}

Currently, a multi pass amplifier based on the $5 \mathrm{~kW}$ disk laser head from Trumpf Laser $\mathrm{GmbH}$ und Co. KG is in construction to further amplify the pulse energy to $200 \mathrm{~mJ}$ at $3 \mathrm{kHz}$. Therefore, just based on simple scaling laws, the pumped disk diameter will be increased by a factor of three from $2.4 \mathrm{~mm}$ to $7.2 \mathrm{~mm}$ and the maximum pump power will be raised from currently $500 \mathrm{~W}$ by a factor of 10 to $5 \mathrm{~kW}$. Expecting $20 \%$ gain per reflection via the disc 10 passes will be realized with additional back reflection to achieve 20 passes through the disk in total. To avoid air fluctuations in front of the pumped disk due to heating, the amplifier will be build in vacuum which allows for stable and reliable $2 \mathrm{f}$-imaging of the multiple passes through the disk without optical breakthrough in air.

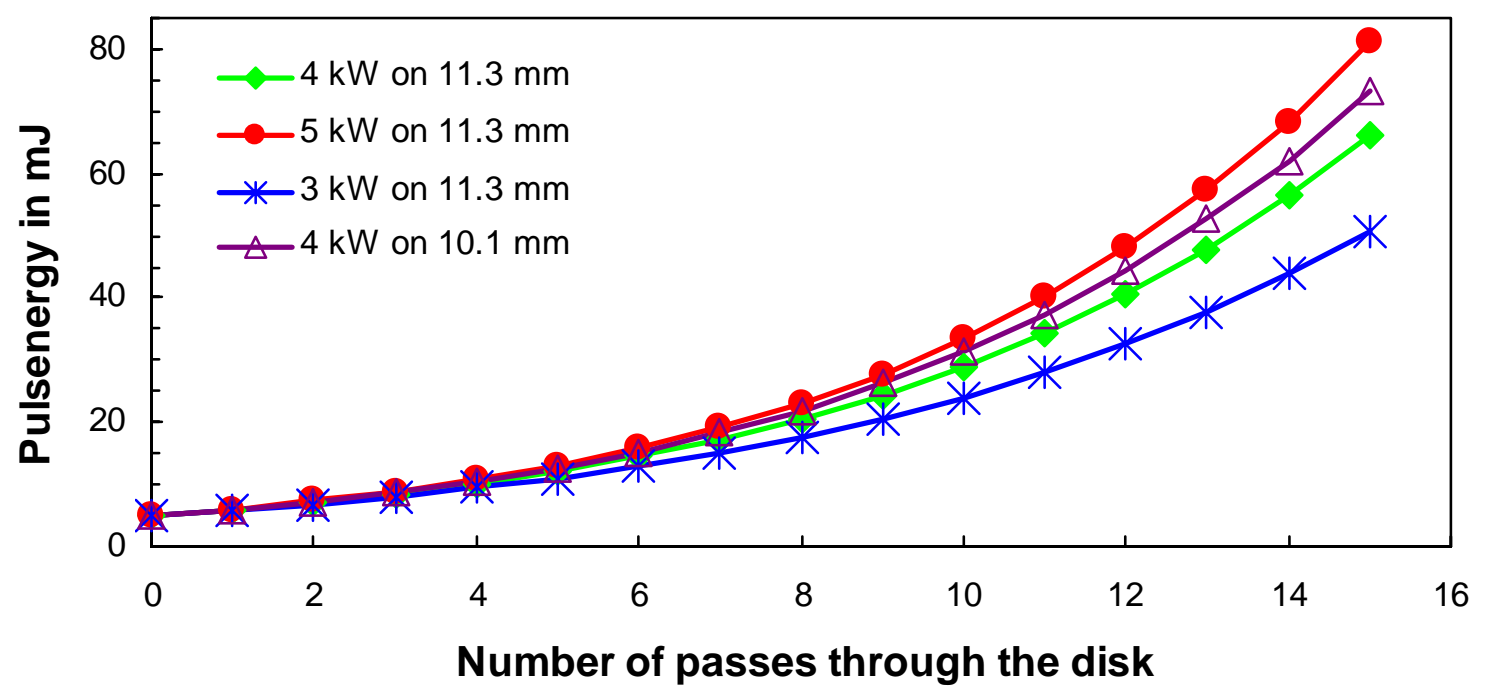

Fig. -1 Calculated amplification for different pump powers on pump spot diameters of 10.1 and 11.3 $\mathrm{mm}$ diameter for an initial seed energy of $5 \mathrm{~mJ}$ at $10 \mathrm{kHz}$.
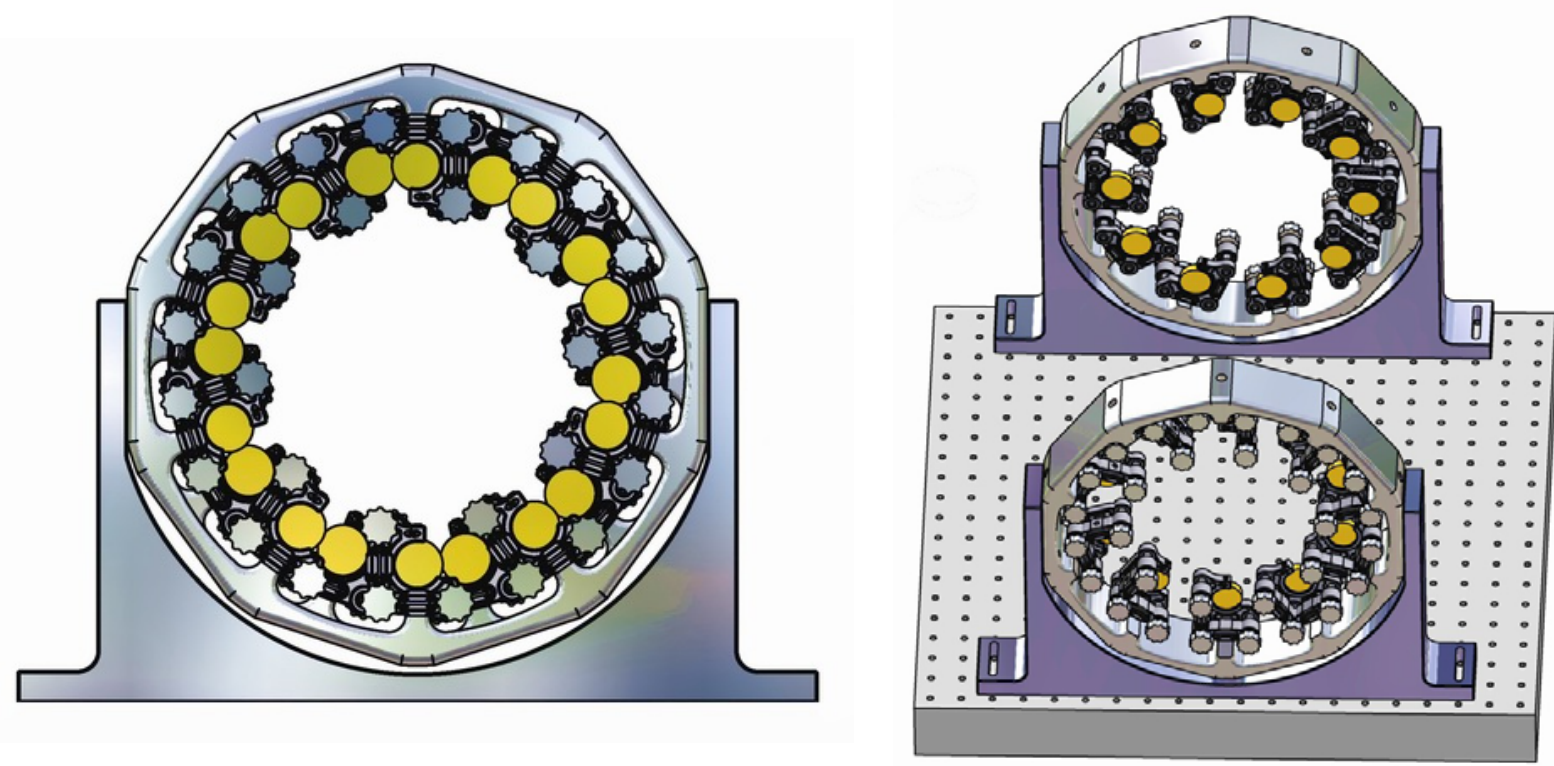

Fig. 9-2 Partial views of the multi pass amplifier system. The left side shows the front view of the imaging mirrors and holders while the right side shows the three dimensional view of the imaging system. 
Originally, the here mentioned multipass amplifier was planned to amplify $5 \mathrm{~mJ}$ pulses to $50 \mathrm{~mJ}$ at a repetition rate of $10 \mathrm{kHz}$. Figure 9-1 shows the calculated amplification for different pump powers and two different beam diameters. With a pump spot diameter of $11.3 \mathrm{~mm}$ and a total pump power of $5 \mathrm{~kW}$, energies of more than $80 \mathrm{~mJ}$ can be expected. The prediction was done by Jochen Speiser (Institut für Strahlwerkzeuge, University Stuttgart, Germany) using the time resolved calculation presented in [82]. Reducing the repetition rate by a factor of three to $3 \mathrm{kHz}$ the expected output energy would increase to approximately $240 \mathrm{~mJ}$ neglecting the losses trough the fluorescence due to the lifetime of the upper laser level of $\sim 1 \mathrm{~ms}$. Considering lower repetition rates around $1 \mathrm{kHz}$ one could expect at least $600 \mathrm{~mJ}$.

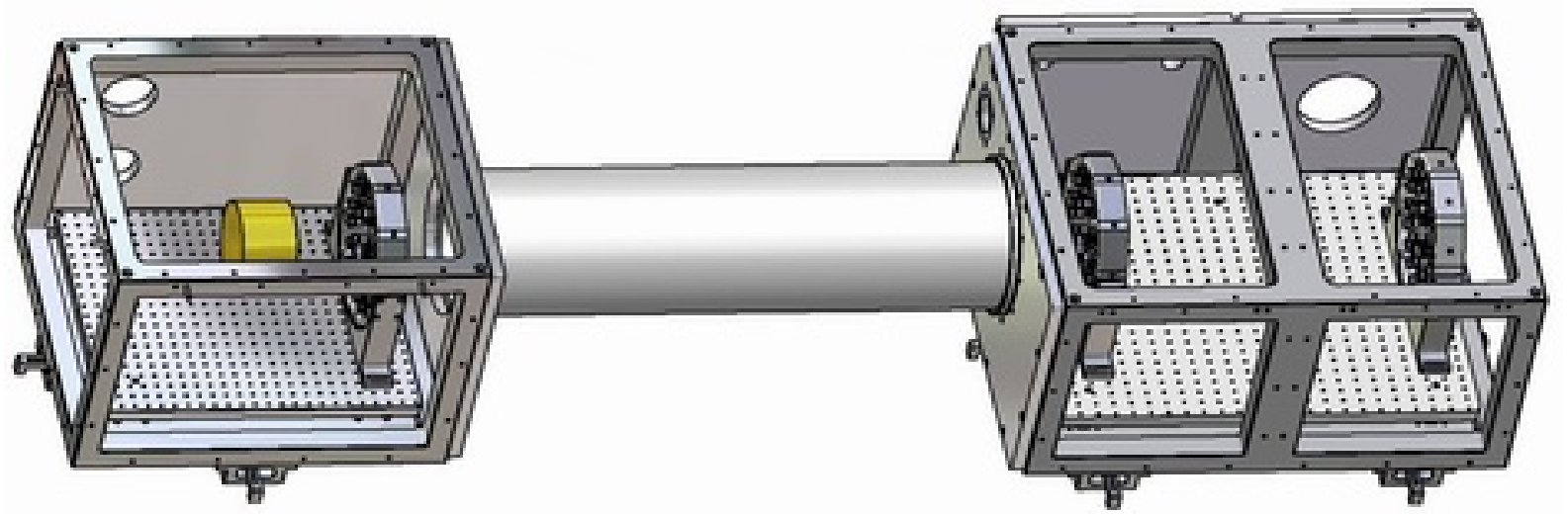

Fig. 9-3 General view of the multipass amplifier system. The vacuum chamber on the left side contains the disk amplifier head together with one mirror holder and in the right vacuum chamber the imaging mirrors shown in the picture above are enclosed.

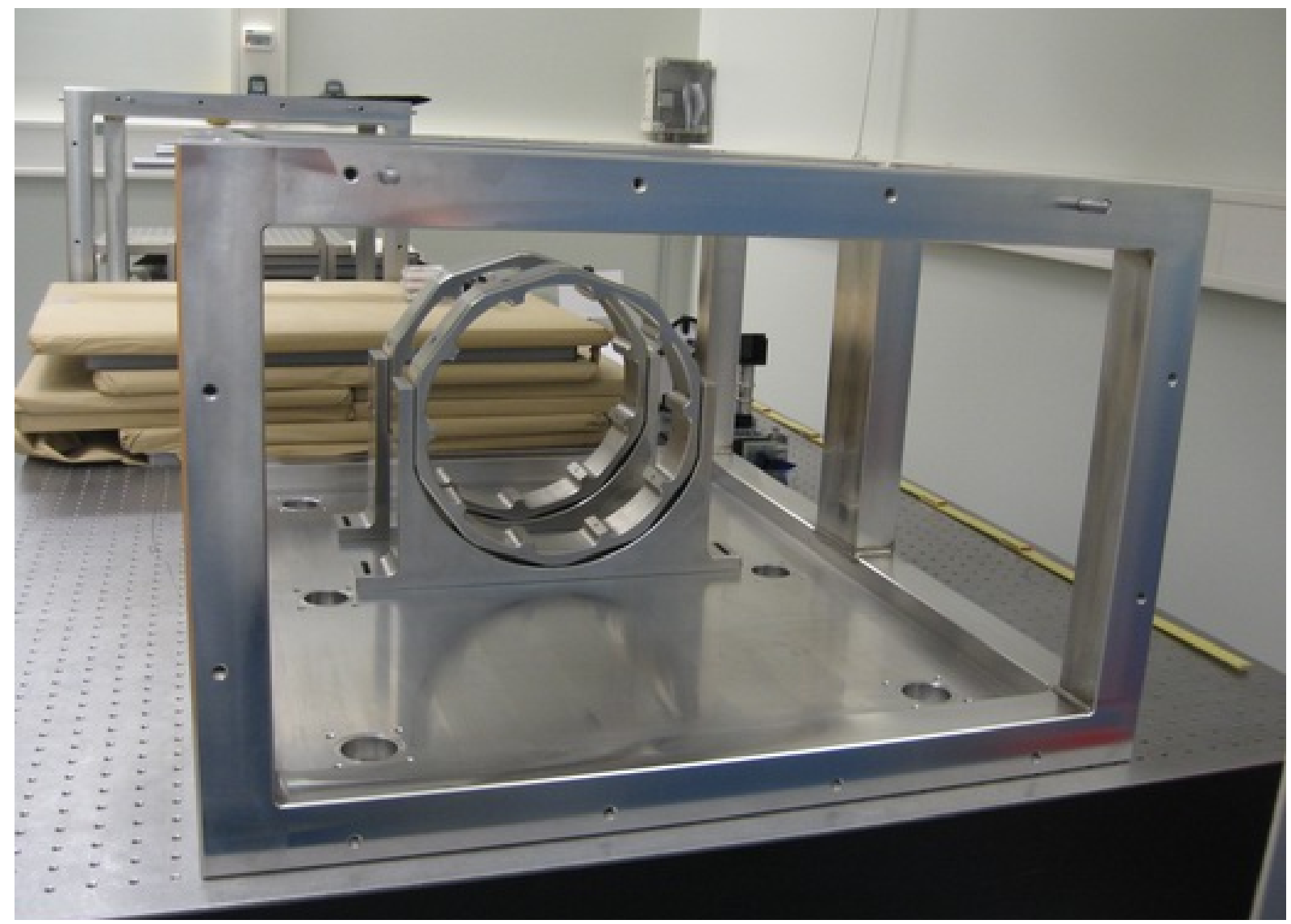

Fig. 9-4 Picture of the imaging vacuum chamber in the especially for the multipass amplifier established laboratory. 
Figure 9-2 shows partial views of the multi pass amplifier system. The left side shows the front view of the imaging mirrors and holders while the right side shows the three dimensional few of the imaging system. The complete apparatus of the constructed multipass amplifier is shown in figure 9-3 and a picture of the imaging vacuum chamber in the especially for the multipass amplifier established laboratory is shown in figure 9-4.

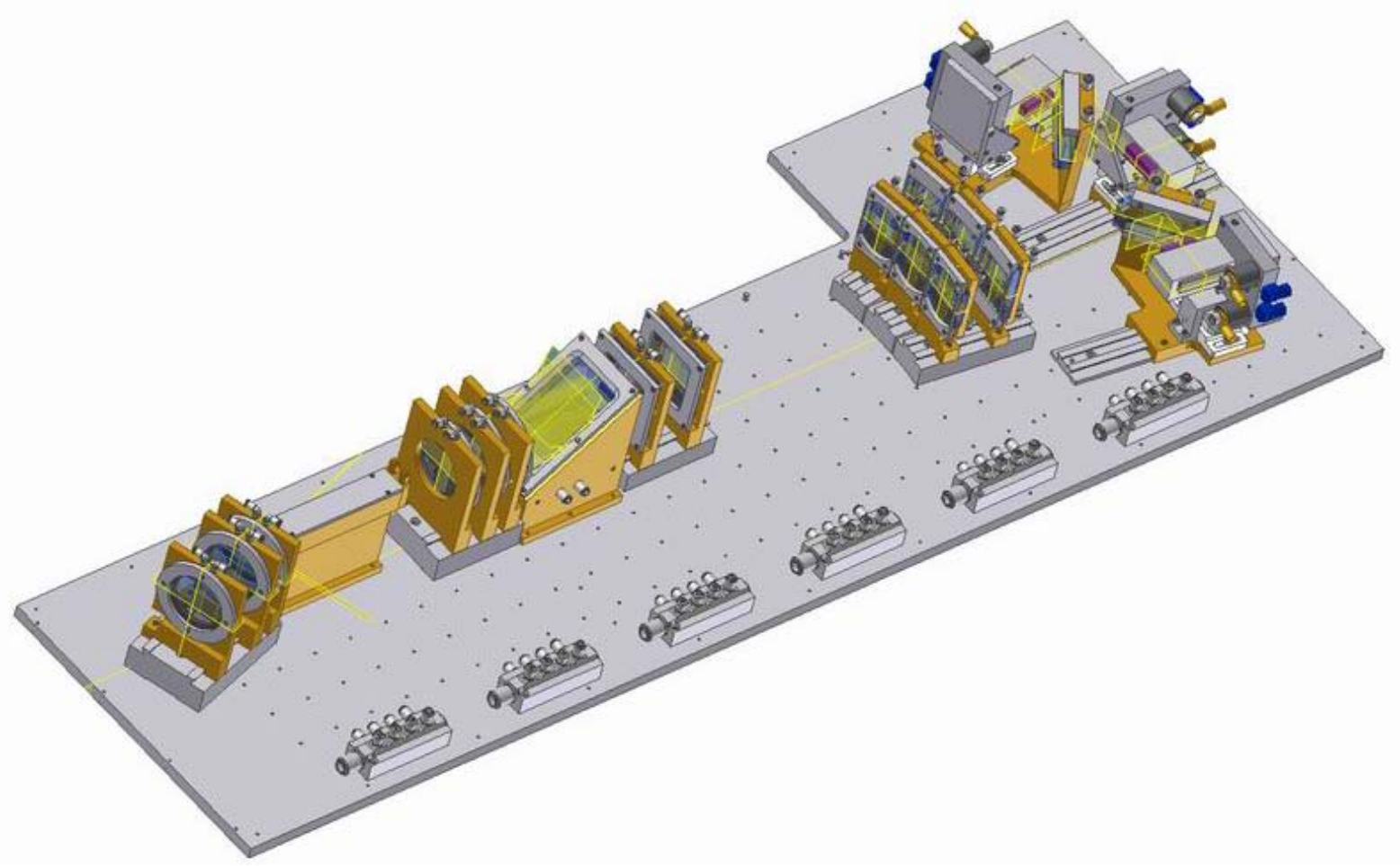

Fig. 9-5 Pump light homogenizer constructed at the IFSW (Technical University Stuttgart) for $5 \mathrm{~kW}$ of pump light provided by two diode stacks from Jenoptik Laserdiode GmbH with $2.5 \mathrm{~kW}$ each.

\begin{tabular}{c|c}
\hline mirror / distance / disk & radius of curvature / distance in $\mathrm{mm}$ \\
\hline disk & -2000 \\
distance & 1750 \\
mirror concave & -1500 \\
distance & 1500 \\
mirror concave & -1500 \\
distance & 1750 \\
mirror concave & -2000 \\
distance & 2000 \\
disk & -2000 \\
\hline
\end{tabular}

Tab. 9-1 Distances and used mirrors in the designed multipass amplifier shown in figure 9-3. Listed from the left to the right side of the shown multipass scheme presented in figure 9-6.

Currently at the completition of this work, all parts of the amplifier system have been already manufactured and are waiting for being assembled. The multipass amplifier will be pumped by the $5 \mathrm{~kW}$ pump light homogenizer designed and constructed at the Institut für Strahlwerkzeuge (IFSW, Technical University Stuttgart) shown in figure 9-5. The two $2.5 \mathrm{~kW}$ di- 
ode stacks from Jenoptik Laserdiode $\mathrm{GmbH}$ are collimated in both axis and focused into a $20 \mathrm{~cm}$ long glass rod and there homogenized via multiple reflection. Followed by a recollimation after three lenses the pump light can be transported via mirrors to the laser amplifier head.

As mentioned above the 10 passes through the disk are realized with an imaging system that steers the beams between the disk and three groups of imaging mirrors. Table 9-1 shows the mirrors and distances calculated for on imaging procedure from the disk back to itself. The beam path of the imaging system is shown in figure 9-6.

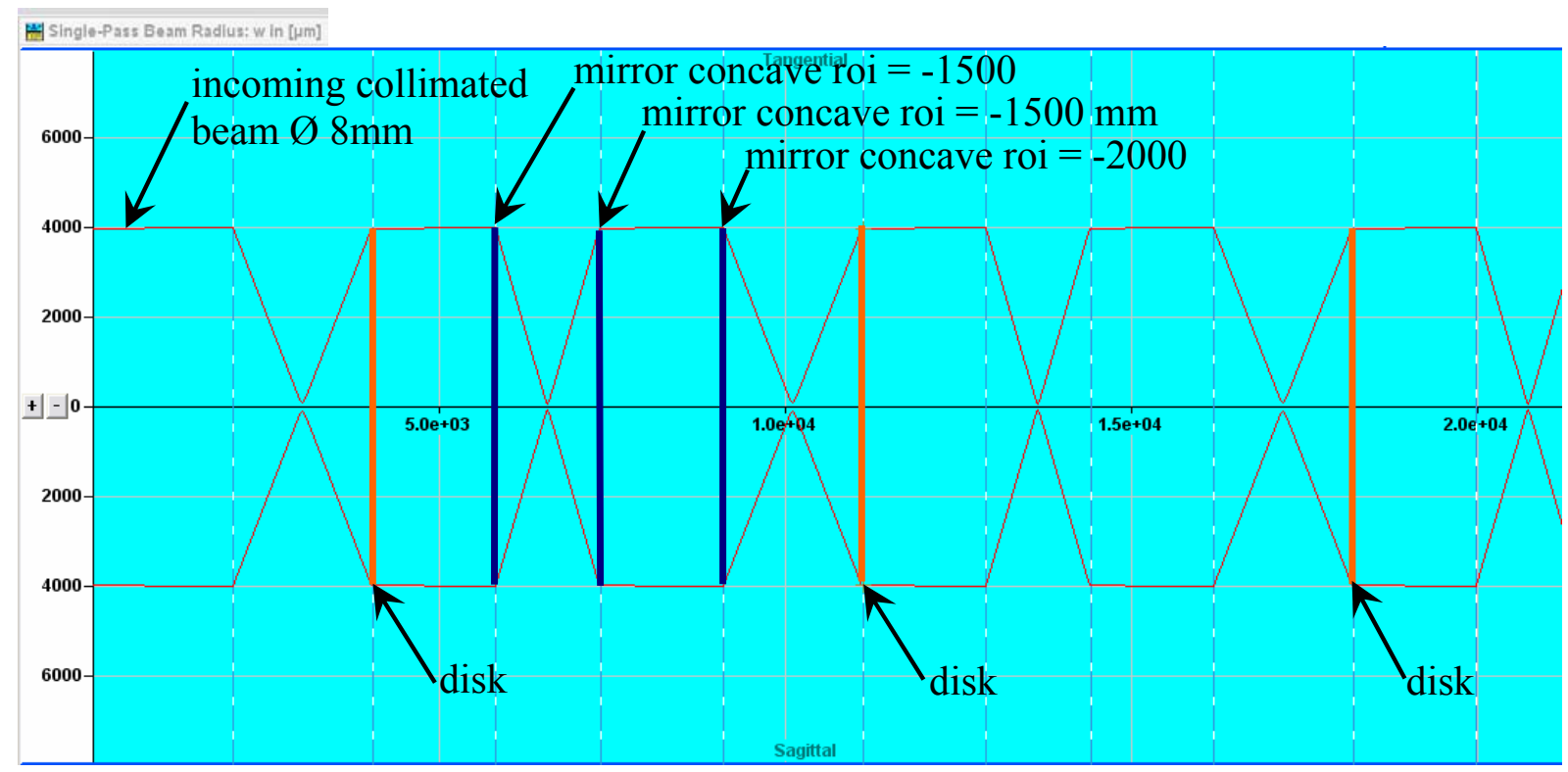

Fig. 9-6 Calculated beam path through the multipass amplifier. A mirror images the incoming $8 \mathrm{~mm}$ diameter beam onto the disk. Three imaging mirrors mounted in the holders shown in figure 94 steer the beam back to the disk. This imaging procedure is repeated 10 times.

To overcome damage problems on the disk increasing the beam diameter by a factor of two and the pump power by a factor of four pulse energies above $2 \mathrm{~J}$ could be achieved in an additional multipass amplifier based on the $12 \mathrm{~kW}$ head from Dausinger + Giesen GmbH shown in figure 2-3. A system delivering pulse energies in the joule range could be based on an optically synchronized regenerative amplifier similar to the system mentioned above and two post amplifiers. Chirped pulse amplification would be required to overcome nonlinear effects in the regenerative amplifier and optical damage on the optics. Stretching the pulses at a bandwidth of $1 \mathrm{~nm}$ (TBL $1.6 \mathrm{ps}$ ) to $200 \mathrm{ps}$ (group delay dispersion of $\sim 1.2 \cdot 10^{8} \mathrm{fs}^{2}$ ) would comply with the above mentioned requirements while still allowing a compact $\sim 2 \mathrm{~m}$ long compressor setup using dielectric reflection gratings with efficiencies at high as $99 \%, 1740$ lines $/ \mathrm{mm}$ on $\sim 300$ to $400 \mathrm{~mm}$ wide gratings. 


\section{Conclusion}

This work presents the experimental realization of different regenerative amplifiers based on an $\mathrm{Yb}$ :YAG gain medium in a thin disk configuration mounted and experimentally investigated in two different laser amplifier heads from the Institut für Strahlwerkzeuge (IFSW) and Trumpf Laser $\mathrm{GmbH} \& \mathrm{Co}$ respectively. Based on the principles of a chirped pulse amplifier a new apparatus was developed aiming at the improvement of several bottlenecks in optical parametric chirped pulse amplifiers.

Summarized, the generation of $25 \mathrm{~mJ}, 1.6 \mathrm{ps}$ pulses delivered in a near-diffraction-limited beam at a wavelength of $1030.2 \mathrm{~nm}$ with a root-mean-square pulse-to-pulse stability of smaller than $0.7 \%$ and a repetition rate of $3.0 \mathrm{kHz}$ from a regenerative chirped pulse amplifier based on a thin-disk Yb:YAG gain medium can be reported.

The implementation of a dispersive delay line led to a significant increase of the pulse energy from $1 \mathrm{~mJ}$ in previous regenerative amplifiers without CPA to $>32 \mathrm{~mJ}$ with a chirped pulse duration of $\sim 200 \mathrm{ps}$ directly available after the regenerative amplifier. The grating based compressor introduced $23 \%$ losses to the pulse energy leading to $25 \mathrm{~mJ}$ pulses with a nearly time bandwidth limited compressed pulse duration of $1.6 \mathrm{ps}$. The peak power after the compression exceeds $15 \mathrm{GW}$. To the best of our knowledge, this is the most powerful multi-kHz regenerative amplifier reported to date.

For the first time a broadly intermittent single-energy regime in the deterministic chaos of a pumped regenerative amplifier could be exploited to produce not only high pulse energies but also a remarkable and superior stability of a root-mean-square fluctuation in pulse energy of $<0.7 \%$. Complete saturation of the gain medium in every second amplification cycle ensures highly stable and reproducible operation of the developed laser system. Measured at the highest pulse energy of $25 \mathrm{~mJ}$ at $3.0 \mathrm{kHz}$ repetition rate $(75 \mathrm{~W}$ of average power) over a period of more than one hour a fluctuation of only $\pm 0.5 \mathrm{~W}$ in average power could be observed and corresponds to a long time stability of $0.66 \%$. This illustrates that highly stable performance of a regenerative amplifier can still be achieved at repetition rates close to the inverse upper state lifetime of the laser gain medium resulting usually in strongly chaotic pulse build up behavior. Utilizing the period doubling regime in the chaotic pulse build-up leads not only to a superior stable performance but also to a very high overall optical efficiency of $26 \%$ including the compression losses. Without compression losses the slope efficiency reaches $53 \%$ for the pulses directly out of the amplifier.

Experiments with an additional nonlinear compression scheme showed the potential of further reduction in pulse duration to at least $450 \mathrm{fs}$. The simple setup consisting of a gas cell and an extra transmission grating based compressor introduces additional $20 \%$ losses in pulse energy but increases the peak power at the same time by a factor of $>3$. In first experiments $8 \mathrm{~mJ}$ pulses at a repetition rate of $3.0 \mathrm{kHz}$ with $\sim 17 \mathrm{GW}$ peak power could be achieved. Adapting the nonlinear compression stage for higher input pulse energies, peak powers as high as $\sim 50 \mathrm{GW}$ seem to be attainable. The pulse energy provided by the regenerative amplifier is high enough to further amplify the pulses at kilohertz repetition rates in a multipass configura- 
tion. Based on simple scalable disk amplifiers higher energies can be reached with a reasonable small amount of passes through the gain medium although the thin disk laser active media provides small overall gain between 10 to $20 \%$. Calculations show that at repetition rates of $10.0 \mathrm{kHz}$ pulse energies of $80 \mathrm{~mJ}$ can be expected. Considering lower repetition rates around $1.0 \mathrm{kHz}$ pulse energies of $600 \mathrm{~mJ}$ seem to be realistic to achieve with an additional post amplifier directly after the regenerative system. 


\section{Outlook}

The following lines summarize the perspective of the presented laser system in the foreseeable time ahead and point out more future-orientated goals that the developed pump source should allow to tackle beside the advantages already mentioned in the introduction.

Employing new few-cycle laser systems generating pulse energies clearly exceeding the $\mathrm{mJ}$ level in the near infrared, the XUV radiation will be shifted towards higher photon energies enabling experimental studies on previously unexplored intermolecular- and atom-dynamics. Reaching the "water window", a wavelength range where water is transparent while carbon structures can be imaged, between 2.2 and $4.4 \mathrm{~nm}$, would allow in situ analysis of biological relevant molecules. The free electron laser in Hamburg (FLASH) generates currently about $50 \mathrm{fs}$ long pulses at wavelengths down to $6.9 \mathrm{~nm}$ with a flux of about $10^{13}$ photons per pulse [115]. Currently these pulses are too long and have too low photon energy to observe fast motions in long biological molecules via diffraction and allow only observation on frozen samples. Therefore short attosecond pulses with high photon flux are required to measure in a single shot the diffraction pattern delivering the information of the molecule allowing observations simultaneously in space and time with sub-atomic resolution. Currently the standard driving source for HHG and pump-probe experiments is a Ti:sapphire laser based laser system (Femtopower) from Femtolasers Produktions $\mathrm{GmbH}$ delivering $10^{7}$ to $10^{8} \mathrm{XUV}$ photons per shot which is currently not sufficient for XUV diffraction measurements on biological tissues. Additional the pulse energy is not efficient to generate two attosecond XUV-beams to use them in pump-probe experiments due to the low count rate monitored in such experiments.

Ishii et el demonstrated in 2005 a near infrared OPA [20] operating at $20 \mathrm{~Hz}$ with an conversion efficiency of $16 \%$ from the $50 \mathrm{~mJ}$ pump pulse at $532 \mathrm{~nm}$ to the compressed $10 \mathrm{fs}$ short NIR pulse amplified to $8 \mathrm{~mJ}$. Although the repetition rate of the presented system is too low for pump-probe experiments it showed the feasibility of a multi-millijoule few-cycle OPA. Such a system pumped with the in this work developed regenerative amplifier could achieve few cycle pulses with pulse energies above $2.5 \mathrm{~mJ}$ at $3 \mathrm{kHz}$ allowing XUV-XUV pump-probe experiments and would abolish the current limitation on observations of systems witch are sensitive to the NIR infrared and the XUV-beam at the same time.

Infrared OPCPAs similar to the one demonstrated from Gu et al. [19] pumped with a powerful ps-laser could possibly generate IR terawatt scale pulses close to the single-cycle regime leading via HHG to broadband XUV pulses close to the atomic unit of time of 24 as.

The simple scalability of thin disk lasers and their elegantly solved thermal issues of high average power lasers could possibly lead to powerful pump sources for petawatt scale lasers with repetition rates in the kilohertz regime leading to table-top free electron accelerators. The theoretical maximum upper diameter for disk based amplifier media is limited by amplified spontaneous emission which travels in a zigzag course between the two surfaces of the disk and was calculated to be $5 \mathrm{~m}$, being able to deliver a maximum output energy of $6500 \mathrm{~J}$ [116]. 


\section{Symbols and abbreviations}

\begin{tabular}{|c|c|c|}
\hline Symbol & Denotation & Unit \\
\hline $\mathrm{a}_{0}$ & Overall Gain & dimensionless \\
\hline $\mathrm{B}$ & B-integral & dimensionless \\
\hline $\mathrm{c}$ & Speed of light & $\mathrm{m} / \mathrm{s}$ \\
\hline $\mathrm{d}$ & Thickness & $\mathrm{m}$ \\
\hline $\mathrm{d}$ & grating constant & $\mathrm{m}$ \\
\hline$d_{\text {eff }}$ & Effective second order nonlinear coefficient & $\mathrm{m} / \mathrm{V}$ \\
\hline e & Electron charge & $\mathrm{C}$ \\
\hline $\mathrm{E}$ & Field strength & $\mathrm{V} / \mathrm{m}$ \\
\hline $\mathrm{E}_{0}$ & Seed energy & $\mathrm{J}$ \\
\hline $\mathrm{E}_{1}$ & Intra cavity energy & $\mathrm{J}$ \\
\hline $\mathrm{f}$ & focal length & $\mathrm{m}$ \\
\hline G & Gain & dimensionless \\
\hline $\mathrm{g}$ & Gain & dimensionless \\
\hline GD & Group delay & $\mathrm{s}$ \\
\hline GDD & Group delay dispersion & $\mathrm{s}^{2}$ \\
\hline $\mathrm{g}_{\mathrm{RT}}$ & Total roundtrip gain & dimensionless \\
\hline$\hbar$ & Reduced Planck constant & $\mathrm{eVs}$ \\
\hline I & Intensity & $\mathrm{W} / \mathrm{cm}^{2}$ \\
\hline $\mathrm{I}_{0}$ & Peak intensity & $\mathrm{W} / \mathrm{cm}^{2}$ \\
\hline $\mathrm{I}_{\mathrm{p}}$ & Ionization potential & $\mathrm{eV}$ \\
\hline $\mathrm{I}_{\mathrm{p}}$ & Pump intensity & $\mathrm{W} / \mathrm{cm}^{2}$ \\
\hline $\mathrm{k}_{\mathrm{i}}$ & Idler wave vectors & $\mathrm{s} / \mathrm{m}$ \\
\hline $\mathrm{k}_{\mathrm{p}}$ & Pump laser wave vectors & $\mathrm{s} / \mathrm{m}$ \\
\hline $\mathrm{k}_{\mathrm{s}}$ & Signal wave vectors & $\mathrm{s} / \mathrm{m}$ \\
\hline $\mathrm{L}$ & Length & $\mathrm{m}$ \\
\hline $\mathrm{L}_{\mathrm{b}}$ & Gain medium length & $\mathrm{m}$ \\
\hline $\mathrm{L}_{\mathrm{PC}}$ & Pockels cell length & $\mathrm{m}$ \\
\hline $\mathrm{m}$ & Electron mass & $\mathrm{MeV} / \mathrm{c}^{2}$ \\
\hline
\end{tabular}




\begin{tabular}{|c|c|c|}
\hline Symbol & Denotation & Unit \\
\hline $\mathrm{n}$ & Number of round trips & dimensionless \\
\hline $\mathrm{n}$ & Total refractive index & dimensionless \\
\hline $\mathrm{n}_{0}$ & Refractive index & dimensionless \\
\hline $\mathrm{n}_{2}$ & Nonlinear refractive index & $\mathrm{cm}^{2} / \mathrm{V}$ \\
\hline $\mathrm{n}_{\mathrm{i}}$ & Refractive index idler & dimensionless \\
\hline $\mathrm{n}_{\mathrm{p}}$ & Refractive index pump laser & dimensionless \\
\hline $\mathrm{n}_{\mathrm{s}}$ & Refractive index signal & dimensionless \\
\hline $\mathrm{n}_{\text {tot }}$ & Total number of roundtrips & dimensionless \\
\hline$\overline{\mathrm{n}}_{2}$ & Nonlinear refractive index & $\mathrm{cm}^{2} / \mathrm{W}$ \\
\hline$\overline{\mathrm{n}}_{2 \mathrm{PC}}$ & Pockels cell refractive index & dimensionless \\
\hline $\mathrm{P}_{\mathrm{cr}, 1}$ & Self trapping power & $\mathrm{W}$ \\
\hline $\mathrm{P}_{\mathrm{cr} 1}$ & Critical Power & 1 \\
\hline $\mathrm{r}_{22}^{\mathrm{T}}$ & Electro-optic coefficient & $\mathrm{m} / \mathrm{V}$ \\
\hline $\mathrm{r}_{\mathrm{b}}$ & Beam diameter in th gain medium & $\mathrm{m}$ \\
\hline $\mathrm{r}_{\mathrm{PC}}$ & Beam diameter in the Pockels cell & $\mathrm{m}$ \\
\hline $\mathrm{t}$ & Time & $\mathrm{s}$ \\
\hline $\mathrm{T}_{0}$ & initial pulse duration & $\mathrm{s}$ \\
\hline $\mathrm{T}_{1}$ & output pulse duration & $\mathrm{s}$ \\
\hline $\mathrm{T}_{\mathrm{FWHM}}$ & Full width half maximum pulse duration & $\mathrm{s}$ \\
\hline $\mathrm{T}_{\text {in }}$ & Input pulse duration & $\mathrm{s}$ \\
\hline $\mathrm{T}_{\text {out }}$ & Output pulse duration & $\mathrm{s}$ \\
\hline $\mathrm{U}_{\mathrm{p}}$ & Ponderomotive potential & $\mathrm{eV}$ \\
\hline $\mathrm{V}_{1 / 4}$ & Quarter-wave voltage & V \\
\hline $\mathrm{w}_{0}$ & Center frequency & $1 / \mathrm{s}$ \\
\hline $\mathrm{z}$ & distance & $\mathrm{m}$ \\
\hline$\beta$ & Angle of incidence & radiant \\
\hline$\beta^{\prime}$ & Diffraction angle & radiant \\
\hline$\beta_{2}$ & Group velocity dispersion & $\mathrm{s}^{2} / \mathrm{m}$ \\
\hline$\chi^{(3)}$ & Third order susceptibility & dimensionless \\
\hline$\varepsilon_{0}$ & Permittivity of free space & $\mathrm{As} / \mathrm{Vm}$ \\
\hline
\end{tabular}




\begin{tabular}{clc} 
Symbol & Denotation & Unit \\
\hline$\Delta \lambda$ & Free spectral range & $\mathrm{m}$ \\
$\Delta \lambda_{\mathrm{g}}$ & Supported bandwidth & $\mathrm{m}$ \\
$\Delta \omega_{\mathrm{g}}$ & Spectral FWHM of the gain curve & $1 / \mathrm{s}$ \\
$\lambda$ & Wavelength & $\mathrm{m}$ \\
$\lambda_{\mathrm{c}}$ & Center wavelength & $\mathrm{m}$ \\
$\lambda_{\mathrm{i}}$ & Idler wave length & $\mathrm{m}$ \\
$\lambda_{\mathrm{p}}$ & Pump wavelength & $\mathrm{m}$ \\
$\lambda_{\mathrm{s}}$ & Signal wavelength & $\mathrm{m}$ \\
$\mu_{0}$ & Permeability of free space & $\mathrm{N} / \mathrm{A}^{2}$ \\
$\tau$ & Pulse duration & $\mathrm{s}$ \\
$\tau_{\mathrm{in}}$ & Input pulse duration & $\mathrm{s}$ \\
$\tau_{\mathrm{out}}$ & Output pulse duration & $\mathrm{s}$ \\
$\omega$ & Frequency & $\mathrm{radiant}$ \\
$\omega_{1}$ & Signal frequency & $1 / \mathrm{s}$ \\
$\omega_{2}$ & Idler frequency & $1 / \mathrm{s}$ \\
$\omega_{3}$ & Pump field of the frequency & $1 / \mathrm{s}$ \\
$\omega_{\mathrm{s}}$ & Signal frequency & $1 / \mathrm{s}$ \\
$\psi$ & Spectral phase & $1 / \mathrm{s}$ \\
& &
\end{tabular}

\section{Abbreviation Denotation}

$\mathrm{Al}_{2} \mathrm{O}_{3} \quad$ Aluminum oxide

ASE Amplified spontaneous emission

BBO $\quad \beta$-barium borate

CEP Carrier-envelope phase

CM Chirped mirror

CP Compensating plate

CPA Chirped pulse amplification

cw Continuous wave

DFG Difference frequency generation

DM Dichroic mirror 


\begin{tabular}{|c|c|}
\hline Abbreviation & Denotation \\
\hline $\mathrm{F}$ & Fluoride \\
\hline FS & Fused silica \\
\hline FWHM & Full width half maxiumum \\
\hline GD & Group delay \\
\hline GDD & Group-delay dispersion \\
\hline GVD & Group-velocity dispersion \\
\hline HHG & High-order harmonic generation \\
\hline HR & High reflecting mirror \\
\hline $\mathrm{HV}$ & high-voltage \\
\hline IFSW & Institut für Strahlwerkzeuge \\
\hline IR & Infrared \\
\hline KGW & Potassium gadolinium tungstate \\
\hline KTP & Potassium Titanyl Phosphate \\
\hline LLNL & Lawrence Livermore National Laboratory \\
\hline $\mathrm{Lu}_{2} \mathrm{O}_{3}$ & Lutetium oxide \\
\hline $\mathrm{Mg}$ & Magnesium \\
\hline $\mathrm{Mg}_{2} \mathrm{~F}_{2}$ & Magnesium fluoride \\
\hline OPA & Optical parametric amplifier \\
\hline OPCPA & Optical parametric chirped pulse amplification \\
\hline OPO & Optical parametric oscillator \\
\hline $\mathrm{PC}$ & Pockels cell \\
\hline PP-MgO:LN & Magnesium-oxide-doped periodically poled lithium niobate \\
\hline RMS & Root-mean-square \\
\hline RTP & Rubidium titanyl phosphate \\
\hline $\mathrm{Sc}_{2} \mathrm{O}_{3}$ & Scandium oxide \\
\hline SF & Self focusing \\
\hline SHG & Second harmonic generation \\
\hline SPM & Self phase modulation \\
\hline TBL & Time bandwidth limited \\
\hline $\mathrm{Te}$ & Tellurium \\
\hline
\end{tabular}




\begin{tabular}{cl} 
Abbreviation & Denotation \\
\hline $\mathrm{TFP}$ & Thin film polarizer \\
$\mathrm{XUV}$ & Extreme ultraviolet \\
$\mathrm{Y}_{2} \mathrm{O}_{3}$ & Yttrium oxide \\
$\mathrm{YAG}$ & Ytterbium aluminum garnet \\
$\mathrm{YALO}$ & Yttrium aluminum oxide \\
$\mathrm{Yb}$ & Ytterbium
\end{tabular}




\section{Bibliography}

[1] T.H. Maiman, “Stimulated Optical Radiation in Ruby,” Nature 187, 493-494 (1960).

[2] W. E. Lamb, “Theory of an optical laser,” Phys. Rev. 134, A1429-A1450 (1964).

[3] L. E. Hargrove, R. L. Fork, and M. A. Pollack, "Locking of He-Ne laser modes induced by synchronous intracavity modulation,” Appl. Phys. Lett. 5, 4-5 (1964).

[4] E. Goulielmakis, M. Schultze, M. Hofstetter, V. S. Yakovlev, J. Gagnon, M. Uiberacker, A. L. Aquila, E. M. Gullikson, D. T. Attwood, R. Kienberger, F. Krausz, and U. Kleineberg, "Single-Cycle Nonlinear Optics," Science 320, 1614-1617 (2008).

[5] A. Baltuška, Takao Fuji, and T. Kobayashi, "Controlling the Carrier-Envelope Phase of Ultrashort Light Pulses with Optical Parametric Amplifiers," Phys. Rev. Lett. 88, 133901-133904 (2002).

[6] F. Krausz, and M. Ivanov, "Attosecond physics," Rev. Mod. Phys. 81, 163-234 (2009).

[7] A. L. Cavalieri, E. Goulielmakis, B. Horvath, W. Helml, M. Schultze, M. Fieß, V. Pervak, L. Veisz, V. S. Yakovlev, M. Uiberacker, A. Apolonski, F. Krausz, R. Kienberger, "Intense 1.5-cycle near infrared laser waveforms and their use for the generation of ultra-broadband soft-x-ray harmonic continua," New J. Phys. 9 , $242-254$ (2007).

[8] P. B. Corkum, "Plasma perspective on strong field multiphoton ionization," Phys. Rev. Lett. 71, 1994-1997 (1993).

[9] Ch. Spielmann, N. H. Burnett, S. Sartania, R. Koppitsch, M. Schnürer, C. Kan, M. Lenzner, P. Wobrauschek, and F. Krausz, "Generation of Coherent X-rays in the Water Window Using 5-Femtosecond Laser Pulses,” Science 278, 661-664 (1997).

[10] R. Kienberger, E. Goulielmakis, M. Uiberacker, A. Baltuška, V. Yakovlev, F. Bammer, A. Scrinzi, T. Westerwalbesloh, U. Kleineberg, U. Heinzmann, M. Drescher, and F. Krausz, “Atomic transient recorder," Nature 427, 817-821 (2004).

[11] M. Schultze, E. Goulielmakis, M. Uiberacker, M. Hofstetter, J. Kim, D. Kim, F. Krausz, and U. Kleineberg, "Powerful 170-attosecond XUV pulses generated with few-cycle laser pulses and broadband multilayer optics," New J. Phys. 9, 243- 253 (2007).

[12] S. Sartania, Z. Cheng, M. Lenzner, G. Tempea, Ch. Spielmann, F. Krausz, and K. Ferencz, "Generation of 0.1-TW 5-fs optical pulses at a 1-kHz repetition rate", Opt. Lett. 22, 1562-1564 (1997). 
[13] M. Nisoli, S. De Silvestri, O. Svelto, R. Szipöcs, K. Ferencz, Ch. Spielmann, S. Sartania, and F. Krausz, "Compression of high-energy laser pulses below 5 fs," Opt. Lett. 22, 522-524 (1997).

[14] J. H. Sung, J. Y. Park, T. Imran, Y. S. Lee, and C. H. Nam, “ Generation of 0.2-TW 5.5-fs optical pulses at $1 \mathrm{kHz}$ using a differentially pumped hollow-fiber chirpedmirror compressor,” Appl. Phys. B 82, 5-8 (2006).

[15] S. Backus, R. Bartels, S. Thompson, R. Dollinger, H. C. Kapteyn, and M. M. Murnane, "High-efficiency, single-stage 7-kHz high-average-power ultrafast laser system," Opt. Lett. 26, 465-467 (2001).

[16] F. Tavella, A. Marcinkevičius, and F. Krausz, "90 mJ parametric chirped pulse amplification of 10 fs pulses, “ Opt. Expr. 14, 12822-12827 (2006).

[17] S. Adachi, N. Ishii, T. Kanai, A. Kosuge, J. Itatani, Y. Kobayashi, D. Yoshitomi, K. Torizuka, and S. Watanabe, "5-fs, multi-mJ, CEP-locked parametric chirped-pulse amplifier pumped by a 450-nm source at 1 kHz," Opt. Expr. 16, 14341-14352 (2008).

[18] S. Witte, R. Th. Zinkstok, A. L. Wolf, W. Hogervorst, W. Ubachs, and K. S. E. Eikema, "A source of 2 terawatt, 2.7 cycle laser pulses based on noncollinear optical parametric chirped pulse amplification,” Opt. Express 14, 8168-8177 (2006).

[19] X. Gu, G. Marcus, Y. Deng, T. Metzger, C. Y. Teisset, N. Ishii, T. Fuji, A. Baltuška, R. Butkus, V. Pervak, H. Ishizuki, T. Taira, T. Kobayashi, R. Kienberger, and F. Krausz, "Generation of carrier-envelope-phase-stable 2-cycle $740-\mu \mathrm{J}$ pulses at $2.1-\mu \mathrm{m}$ carrier wavelength,” Opt. Express 17, $62-69$ (2009).

[20] N. Ishii, L. Turi, V. S. Yakovlev, T. Fuji, F. Krausz, A. Baltuška, R. Butkus, G. Veitas, V. Smilgevicius, R. Danielius, and A. Piskarskas, "Multimillijoule chirped parametric amplification of few-cycle pulses," Opt. Lett. 30, 567-569, (2005).

[21] F. Tavella, Y. Nomura, L. Veisz, V. Pervak, A. Marcinkevičius, and F. Krausz, "Dispersion management for a sub-10-fs, 10 TW optical parametric chirped-pulse amplifier," Opt. Lett. 32, 2227-2229 (2007).

[22] N. Ishii, C. Y. Teisset, T. Fuji, S. Köhler, K. Schmid, L. Veisz, A. Baltuška, and F. Krausz, "Seeding of an eleven femtosecond optical parametric chirped pulse amplifier and its $\mathrm{Nd}^{3+}$ picosecond pump laser from a single broadband Ti:sapphire oscillator," IEEE J. Sel. Top. Quantum Electron. 12, 173-180 (2006).

[23] C. Dorrer, I. A. Begishev, A. V. Okishev, and J. D. Zuegel, "High-contrast opticalparametric amplifier as a front end of high-power laser systems," Opt. Lett. 32, 21432145 (2007). 
[24] R. Szipocs, K. Ferencz, C. Spielmann, and F. Krausz, "Chirped multilayer coatings for broadband dispersion control in femtosecond lasers,” Opt. Lett. 19, 201-203 (1994).

[25] B. C. Stuart, M. D. Feit, S. Herman, A. M. Rubenchik, B. W. Shore, and M. D. Perry, "Nanosecond-to-femtosecond laser-induced breakdown in dielectrics," Phys. Rev. B 53, 1749 - 1761 (1996).

[26] G. Cerullo, and S. De Silvestri, "Ultrafast optical parametric amplifiers," Rev. Sci. Instrum. 74, 1 (2003).

[27] D. Strickland, and G. Mourou, "Compression of amplified chirped optical pulses," Opt. Comm. 56, 219-221 (1985).

[28] A. Giesen, and J. Speiser, "Fifteen Years of Work on Thin-Disk Lasers: Results and Scaling Laws,” IEEE J. Sel. Top. Quantum Electron. 13, 598-609 (2007).

[29] C. Stolzenburg, and A. Giesen, "Picosecond regenerative Yb:YAG thin disk amplifier at $200 \mathrm{kHz}$ repetition rate and $62 \mathrm{~W}$ output power," in Advanced Solid-State Photonics, Technical Digest (Optical Society of America, 2007), paper MA6.

[30] T. Clausnitzer, J. Limpert, K. Zöllner, H. Zellmer, H.-J. Fuchs, E.-B. Kley, A. Tünnermann, M. Jupé, and D. Ristau, "Highly Efficient Transmission Gratings in Fused Silica for Chirped-Pulse Amplification Systems,” Appl. Opt. 42, 6934-6938 (2003).

[31] N. Destouches, A. Tishchenko, J. Pommier, S. Reynaud, O. Parriaux, S. Tonchev, and M. Ahmed, "99\% efficiency measured in the -1 st order of a resonant grating," Opt. Express 13, 3230-3235 (2005).

[32] D. Gloge, and R. Roldan, "Investigation of low power laser signals with picosecond resolution,” Appl. Phys. Lett. 14, 3 (1969).

[33] D. J. Kuizenga, and A. E. Siegman, "FM and AM mode locking of the homogeneous laser - Part I: Theory”, IEEE J. Quantum Electron. 6, 694-708 (1970).

[34] D. J. Kuizenga, and A. E. Siegman, "FM and AM mode locking of the homogeneous laser - Part II: experimental results in a Nd:YAG laser with internal FM modulation", IEEE J. Quantum Electron. 6, 709-715 (1970).

[35] T. Fuji, A. Unterhuber, V. S. Yakovlev, G. Tempea, A. Stingl, and F. Krausz, W. Drexler, "Generation of smooth, ultra-broadband spectra directly from a prism-less Ti:sapphire laser,” Appl. Phys. B 77, 125-128 (2003).

[36] J J. Neuhaus, D. Bauer, Ch. Scharfenberg, J. Kleinbauer, S. Weiler, A. Killi, D. H. Sutter, T. Dekorsy, " Ultrafast Yb:YAG thin-disk oscillator with pulse energies exceeding $25 \mu \mathrm{J}$ suitable for efficient ablation with negligible heat affects, “ in Proceedings of SPIE Volume: 7203, 72030T (2009). 
[37] Koechner, W., Solid-State Laser Engineering, $5^{\text {th }}$ edition, Springer, Berlin, 1999

[38] J. E. Murray, and W.H. Lowdermilk, "Nd:YAG regenerative amplifier," J. Appl. Phys. 51, 3548-3555 (1980).

[39] D. Nickel, C. Stolzenburg, A. Beyertt, A. Giesen, J. Häußermann, F. Butze, and M. Leitner, "200 kHz electro-optic switch for ultrafast laser systems," Rev. Sci. Instrum. 76, 0331111-0331117 (2005).

[40] Y. Akahane, M. Aoyama, K. Ogawa, K. Tsuji, S. Tokita, J. Kawanaka, H. Nishioka, and K. Yamakawa, "High-energy, diode-pumped, picosecond Yb:YAG chirped-pulse regenerative amplifier for pumping optical parametric chirped-pulse amplification,“ Opt. Lett. 32, 1899-1901 (2007).

[41] B. Beyertt, D. Nickel, and A. Giesen, "Femtosecond thin-disk Yb:KYW regenerative amplifier," Appl. Phys. B 80, 1-6 (2005).

[42] G. Vaillancourt, T. B. Norris, J. Scott Coe, P. Bado, and G. A. Mourou, "Operation of a 1-kHz pulse-pumped Ti:sapphire regenerative amplifier”, Opt. Lett. 15, 317 (1990).

[43] W. H. Lowdermilk, and J. E. Murray, "The multipass amplifier: Theory and numerical analysis”, J. Appl. Phys. 51, 2436- 2444 (1980).

[44] Reider, G. A., Photonik. Eine Einführung in die Grundlagen, Springer Verlag, Wien, 1997.

[45] C. C. Wang and G.W. Racette, "Measurement of parametric gain accompanying optical difference frequency generation", Appl. Phys. Lett. 6, 169-171 (1965).

[46] N. M. Kroll, "Parametric amplification in spatially extended media and application to the design of tuneable oscillators at optical frequencies", Phys. Rev. 127, 1207-1211 (1962).

[47] T.Wilhelm, J. Piel, and E. Riedle, "Sub-20-fs pulses tunable across the visible from a blue-pumped single-pass noncollinear parametric converter”, Opt. Lett. 22, 1494-1496 (1997).

[48] J. Piel, M. Beutter, and E. Riedle, "20-50-fs pulses tunable across the near infrared from a blue-pumped noncollinear parametric amplifier", Opt. Lett. 25, 180-182 (2000).

[49] A. Dubietis, G. Jonusauskas, and A. Piskarskas, "Powerful femtosecond pulse generation by chirped and stretched pulse parametric amplification in BBO crystal", Opt. Comm. 88, 437-440 (1992). 
[50] A. Piskarskas, A. Stabinis, and A. Yankauskas, "Phase phenomena in parametric amplifiers and generators of ultrashort light pulses”, Sov. Phys. Usp. 29, 869879 (1986).

[51] D. Xue, K. Betzler, H. Hesse and D. Lammers, "Nonlinear optical properties of borate crystals", Sol. State Comm. 114, 21-25 (2000).

[52] I. N. Ross, P. Matousek, M. Towrie, A. J. Langley, and J. L. Collier, "The prospects for ultrashort pulse duration and ultrahigh intensity using optical parametric chirped pulse amplifiers", Opt. Comm. 144, 125-133, (1997).

[53] I. Jovanovic, C.A. Ebbers, and C.P.J. Barty, "Hybrid chirped-pulse amplification", Opt. Lett. 27, 1622-1624 (2002).

[54] R. Butkus, R. Danielius, A. Dubietis, A. Piskarskas and A. Stabinis, "Progress in chirped pulse optical parametric amplifiers”, Appl. Phys. B 79, 693-700 (2004).

[55] C. Manzoni, D. Polli, G. Cirmi, D. Brida, S. De Silvestri, and G. Cerullo, "Tunable few-optical-cycle pulses with passive carrier-envelope phase stabilization from an optical parametric amplifier", Appl. Phys. Lett. 90, 171111 1-3 (2007).

[56] A. Baltuška, T. Fuji and T. Kobayashi, "Self-referencing of the carrier-envelope slip in a 6-fs visible parametric amplifer”, Opt. Lett. 27, 1241-1243 (2002).

[57] P. B. Corkum, and F. Krausz, “Attosecond science,” Nature Phys. 3, 381-387 (2007).

[58] E. Goulielmakis, V. S. Yakovlev, A. L. Cavalieri, M. Uiberacker, V. Pervak, A. Apolonski, R. Kienberger, U. Kleineberg, and F. Krausz, "Attosecond Control and Measurement: Lightwave Electronics,” Science 317, 769-775 (2007).

[59] I. P. Christov, M. M. Murnane, and H. C. Kapteyn, "High-Harmonic Generation of Attosecond Pulses in the "Single-Cycle" Regime," Phys. Rev. Lett. 78, 1251 (1997).

[60] C. Winterfeldt, C. Spielmann, and G. Gerber, "Optimal control of high-harmonic generation," Rev. Mod. Phys. 80, 117-140 (2008).

[61] M. Lewenstein, P. Balcou, M. Y. Ivanov, A. L'Huillier, and P. Corkum, "Theory of high-harmonic generation by low-frequency laser fields," Phys. Rev. A 49, 2117-2132 (1994).

[62] P. Corkum, "Plasma perspective on strong-field multiphoton ionization," Phys. Rev. Lett. 71, 1994-1997 (1993).

[63] M. Lewenstein, P. Balcou, M. Y. Ivanov, A. L'Huillier, and P. Corkum," Theory of high-harmonic generation by low-frequency laser fields," Phys. Rev. A 49, 2117-2132 (1994). 
[64] J. L. Krause, K. J. Schafer, and K. C. Kulander, "High-order harmonic generation from atoms and ions in the high-intensity regime," Phys. Rev. Lett. 68, 3535-3538 (1992).

[65] P. Colosimo, G. Doumy, C. I. Blaga, J. Wheeler, C. Hauri, F. Catoire, J. Tate, R. Chirla, A. M. March, G. G. Paulus, H. G. Muller, P. Agostini, and L. F. Dimauro, "Scaling strong-field interactions towards the classical limit," Nature Phys. 4, 386-389 (2008).

[66] J. Tate, T. Auguste, H. G. Muller, P. Salieres, P. Agostini, and L. F. DiMauro, "Scaling of wave-packet dynamics in an intense midinfrared field," Phys. Rev. Lett. 98 (2007).

[67] A. Gordon and F. X. Kärtner, "Scaling of keV HHG photon yield with drive wavelength," Opt. Express 13, 2491-2497 (2005).

[68] T. Fuji, N. Ishii, C. Y. Teisset, X. Gu, T. Metzger, A. Baltuška, N. Forget, D. Kaplan, A. Galvanauskas, and F. Krausz, "Parametric amplification of few-cycle carrier-

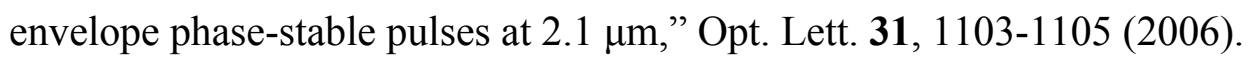

[69] P. Agostini, and L. DiMauro, "The physics of attosecond light pulses," Rep. Prog. Phys 67, 6, 813-855 (2004).

[70] A. Gordon, and F. Kärtner, "Scaling of keV HHG Photon Yield with Drive Wavelength,” Opt. Exp. 13, 8, 2941-2947 (2005).

[71] J. Tate, T. Auguste, H. G. Muller, P. Salières, P. Agostini, and L. F. Di Mauro, "Scaling of Wave-Packet Dynamics in an Intense Midinfrared Field. Phys. Rev. Lett. 98, 013901 1-4 (2007).

[72] Shan, and Z. Chang. "Dramatic extension of the high-order harmonic cutoff by using a long-wavelength pump,” Phys. Rev. A 65, 011804 1-4 (2002).

[73] K. Schiessl, K. Ishikawa, E. Persson, and J. Burgdörfer, "Quantum path interference in the wavelength dependence of high-harmonic generation," Phys. Rev. Lett. 99, 253903 (2007).

[74] T. Ditmire, J. K. Crane, H. Nguyen, L. B. DaSilva, and M. D. Perry, "Energy-yield and conversion-efficiency measurements of high-order harmonic radiation," Phys. Rev. A 51, R902-R905 (1995).

[75] M. Protopapas, C. Keitel, and P. Knight, "Atomic physics with super-high intensity lasers,” Rep. Prog. Phys 60, 389-486 (1997).

[76] T. Brabec, and F. Krausz, "Intense few-cycle laser fields: Frontiers of nonlinear optics,” Rev. Mod. Phys. 72, 545-591 (2000). 
[77] S. Brawer, "Phenomenological theory of laser damage in insulators," Phys. Rev. B 20, 3422-3441 (1979).

[78] A. Killi, I. Zawischa, D. Sutter, J. Kleinbauer, S. Schad, J. Neuhaus, and C. Schmitz, "Current status and development trends of disk laser technology," in Proceedings of SPIE, Volume 6871, pp. 68710L-68710L-10 (2008).

[79] T.Y. Fan," Heat generation in Nd:YAG and Yb:YAG," IEEE J. Quantum Electron. 29, 1457-1459 (1993).

[80] S. V. Marchese, C. R. E. Baer, R. Peters, C. Kränkel, A. G. Engqvist, M. Golling, D. J. H. C. Maas, K. Petermann, T. Südmeyer, G. Huber, and U. Keller, "Efficient femtosecond high power $\mathrm{Yb}: \mathrm{Lu}_{2} \mathrm{O}_{3}$ thin disk laser,” Opt. Express 15, 16966-16971 (2007).

[81] A. Giesen, H. Hiige, P. A. Voss, K. Wittig, U. Brauch, and H. Opower, "Scalable Concept for Diode-Pumped High-Power Solid-State Lasers," Appl. Phys. B 58, 365372 (1994).

[82] Jochen Speiser, "Thin Disk Laser - Energy Scaling," Laser Physics 19, 274-280 (2009).

[83] R.T. Zinkstok, S. Witte, W. Hogervorst, and K.S.E. Eikema, "High-power parametric amplification of 11.8 fs laser pulses with carrier-envelope phase control," Opt. Lett. 30, 78-80, (2004).

[84] S. Witte, R. T. Zinkstok, W. Hogervorst, and K. S. E. Eikema, "Generation of fewcycle terawatt light pulses using optical parametric chirped pulse amplification," Opt. Express 13, 4903-4908, (2005).

[85] M. J. W. Rodwell, D. M. Bloom, and K. J. Weingarten, "Subpicosecond laser timing stabilization,” IEEE J. Quantum Electron. 25, 817-827, (1989).

[86] C. Y. Teisset, N. Ishii, T. Fuji, T. Metzger, S. Köhler, R. Holzwarth, A. Baltuška, A. M. Zheltikov, and F. Krausz, "Soliton-based pump-seed synchronization for few-cycle OPCPA,” Opt. Express 13, 6550-6557 (2005).

[87] T. Fuji, A. Unterhuber, V. S. Yakovlev, G. Tempea, A. Stingl, and F. Krausz, W. Drexler, "Generation of smooth, ultra-broadband spectra directly from a prism-less Ti:sapphire laser,” Appl. Phys. B 77, 125-128 (2003).

[88] F. Krausz, M.E. Fermann, T. Brabec, P. F. Curley, M. Hofer, M. H. Ober, C. Spielmann, E. Wintner, and A. J. Schmidt, "Femtosecond solid-state lasers", IEEE J. Quantum Electron. 28, 2097-2122 (1992). 
[89] T. Fuji, J. Rauschenberger, C. Gohle, A. Apolonski, T. Udem, V. S. Yakovlev, G. Tempea, T. W. Hänsch, and F. Krausz, "Attosecond control of optical waveforms," New J. Phys. 7, 116-124 (2005).

[90] Diels, J.-C., and Rudolph, W., Ultrashort Laser Pulse Phenomena, $2^{\text {nd }}$ edition, Academic Press, Amsterdam, 2006

[91] R. DeSalvo, A. A. Said, D. J. Hagan, E. W. Van Stryland, and M. Sheik-Bahae, "Infrared to ultraviolet measurements of two-photon absorption and $\mathrm{n}_{2}$ in wide bandgap solids,” IEEE J. Quantum Electron. 32, 1324-1333 (1996).

[92] Y. Shimoji, A. T. Fay, R. S. F. Chang, and N. Djeu, "Direct measurement of the nonlinear refractive index of air,” J. Opt. Soc. Am. B 6, 1994-1998 (1989).

[93] R. Adair, L. L. Chase, and S. A. Payne, "Nonlinear refractive index of optical crystals," Phys. Rev. B 39, 3337-3350 (1989).

[94] Agrawal, G. P., Nonlinear Fiber Optics, Academic Press, London, 1989

[95] A. Baltuška, Z. Wei, M. S. Pshenichnikov, and D. A. Wiersma, "Optical pulse compression to $5 \mathrm{fs}$ at a 1-MHz repetition rate," Opt. Lett. 22, 102-104 (1997).

[96] D. Nickel, C. Stolzenburg, A. Beyertt, and A. Giesen, “200 kHz electro-optic switch for ultrafast laser systems, ’Rev. Sci. Instrum. 76, 033111-1-033111-7 (2005).

[97] Siegman, A. E., Lasers, University Science Books, Sausalito, 1986

[98] M. Abarkan, J. P. Salvestrini, M. D. Fontana, and M. Aillerie, "Frequency and wavelength dependences of electro-optic coefficients in inorganic crystals," Appl. Phys. B 76, 765-769 (2003).

[99] G. D. Goodno, Z. Guo, R. J. D. Miller, I. J. Miller, J. W. Montgomery, S. R. Adhav, and R. S. Adhav, "Investigation of beta- $\mathrm{BaB}_{2} \mathrm{O}_{4}$ as a $\mathrm{Q}$ switch for high power applications,” Appl. Phys. Lett. 66, 1575-1578 (1995).

[100] H. Nakatani, W. R. Bosenberg, L. K. Cheng, and C. L. Tang, "Laser-induced damage in beta-barium metaborat, "Appl. Phys. Lett. 53, 2587-2589 (1988).

[101] C. Hönninger, I. Johannsen, M. Moser, G. Zhang, A. Giesen and U. Keller, "Diodepumped thin-disk Yb:YAG regenerative amplifier," Appl. Phys. B 65, 423-426 (1997).

[102] D. Strickland, and G. Mourou, "Compression of amplified chirped optical pulses," Opt. Comm. 56, 219-221 (1985). 
[103] O. E. Martinez, "3000 times grating compressor with positive group velocity dispersion: Application to fiber compensation in 1.3-1-6 $\mu \mathrm{m}$ region,” IEEE J. Quantum Electron. 23, 59-64 (1987).

[104] Müller, D., Pulsenergiestabilität bei regenerativen Kurzpulsverstärkern im Scheibenlaserdesign, Herbert Utz Verlag, München, 2005

[105] K. I. Martin, W. A. Clarkson, and D. C. Hanna, "High-power single-frequency operation, at $1064 \mathrm{~nm}$ and $1061.4 \mathrm{~nm}$ of a Nd:YAG ring laser end-pumped by a beamshaped diode bar," Opt.Comm. 135, 89-92 (1997).

[106] M. D. Skeldon, and S. T. Bui, "Temporal pulse-width control of a regenerative amplifier with intracavity etalons," in Proceedings of SPIE 1869, 120-127 (1993).

[107] J. Desbois, F. Gires, and P. Turnois, "A new approach to picosecond laser pulse analysis shaping and coding," IEEE J. Quantum Electron. 9, 213-218 (1973).

[108] Klein, M., V., and Furtac, E., Optics, John Wiley \& Sons, New-York, 1986

[109] Hecht, E. Optik, $3^{\text {rd }}$ edition, Oldenbourg, München, 2001

[110] J. Dörring, A. Killi, U. Morgner, A. Lang, M. Lederer, and D. Kopf, "Period doubling and deterministic chaos in continuously pumped regenerative amplifiers," Opt. Express 12, 1759-1768 (2004).

[111] M. Grishin, V. Gulbinas, and A. Michailovas, "Dynamics of high repetition rate regenerative amplifiers," Opt. Express 15, 9434-9443 (2007).

[112] M. Nisoli, S. De Silvestri, O. Svelto, R. Szipöcs, K. Ferencz, Ch. Spielmann, S. Sartania, and F. Krausz, "Compression of high-energy laser pulses below 5 fs," Opt. Lett. 22, 522-524 (1997).

[113] T. Südmeyer, F. Brunner, E. Innerhofer, R. Paschotta, K. Furusawa, J. C. Baggett, T. M. Monro, D. J. Richardson, and U. Keller, "Nonlinear femtosecond pulse compression at high average power levels by use of a large-mode-area holey fiber," Opt. Lett. 28, 1951-1953 (2003).

[114] E. Goulielmakis, S. Koehler, B. Reiter, M. Schultze, A. J. Verhoef, E. E. Serebryannikov, A. M. Zheltikov, and F. Krausz, "Ultrabroadband, coherent light source based on self-channeling of few-cycle pulses in helium,” Opt. Lett. 33, 1407-1409 (2008).

[115] K. Tiedtke, A. Azima, N. Bargen, L. Bittner, S. Bonfigt, S. Düsterer, B. Faatz, U. Frühling, M. Gensch., C. Gerth., N. Guerassimova, U. Hahn, T. Hans, M. Hesse, K. Honkavaar, U. Jastrow, P. Juranic, S. Kapitzki, B. Keitel, T. Kracht, M. Kuhlmann, W. B. Li, M. Martins, T. Núñez, E. Plönjes, H. Redlin, E. L. Saldin, E. A. Schneidmiller, J. R. Schneider, S. Schreiber, N. Stojanovic, F. Tavella1, S. Toleikis, R. Treusch, H. 
Weigelt, M. Wellhöfer, H. Wabnitz, M. V. Yurkov, and J. Feldhaus, "The soft x-ray free-electron laser FLASH at DESY: beamlines, diagnostics and end-stations," New J. Phys. 11023029 (2009).

[116] J. Speiser, “Thin Disk Laser-Energy Scaling,” Laser Physics 19, 274-280 (2009). 


\section{Addendum}

\section{List of publications}

[1] T. Metzger, J. Rauschenberger, A. Apolonski, K. Schmid, A. Baltuška, and F. Krausz, "Ultrakurze Laserpulse,” Laser Tech. J. 4, 27-33 (2005).

[2] E. E. Serebryannikov, A. M. Zheltikov, N. Ishii, C. Y. Teisset, S. Köhler, T. Fuji, T. Metzger, F. Krausz, and A. Baltuška, "Soliton self-frequency shift of 6-fs pulses in photonic-crystal fibers," Appl. Phys. B 81, 585-588 (2005).

[3] C. Y. Teisset, N. Ishii, T. Fuji, T. Metzger, S. Köhler, R. Holzwarth, A. Baltuška, A. M. Zheltikov, and F. Krausz, "Soliton-based pump-seed synchronization for few-cycle OPCPA,” Opt. Exp. 13, 6550-6557 (2005).

[4] E. E. Serebryannikov, A. M. Zheltikov, N. Ishii, C. Teisset, St. Köhler, T. Fuji, Th. Metzger, F. Krausz, A. Baltuška, "Nonlinear-optical spectral transformation of fewcycle laser pulses in photonic-crystal fibers," Phys. Rev. E 72, 056603-1-056603-6 (2005).

[5] C. Y. Teisset, N. Ishii, T. Fuji, T. Metzger, S. Koehler, A. Baltuska, and F. Krausz, "All-Optical Pump-Seed Synchronization for Few-Cycle OPCPA," in Conference on Lasers and Electro-Optics/Quantum Electronics and Laser Science and Photonic Applications Systems Technologies, Technical Digest (CD) (Optical Society of America, 2005), paper CMB3.

[6] N. Ishii, A. Baltuška, C.Y. Teisset, T. Fuji, T. Metzger, S. Köhler, G. Veitas, R. Danielius, A.M. Zheltikov, and F. Krausz, "Passively synchronized multimillijoule optical parametric chirped pulse amplifier with a positive-dispersion sub-10-fs pulse compression," in Conference on Lasers and Electro-Optics Europe, 2005. CLEO/Europe, 349 (2005).

[7] T. Fuji, N. Ishii, A. Baltuska, T. Metzger, F. Krausz, and A. Galvanauskas, "Efficient 50-THz-wide phase-stabilized chirped parametric amplification at $2.1 \mu \mathrm{m}$," in Conference on Lasers and Electro-Optics Europe, 2005. CLEO/Europe, 372 (2005).

[8] T. Fuji, N. Ishii, T. Metzger, L. Turi, A. Baltuska, F. Krausz, and A. Galvanauskas, "Infrared optical parametric chirped pulse amplifier for high harmonic generation", in Conference on Ultrafast Optics V, Sep. 2005, Nara, Japan.

[9] T. Fuji, N. Ishii, Th. Metzger, L. Turi, A. Baltuska, F. Krausz, and A. Galvanauskas, "Infrared chirped parametric amplification with self-stabilized carrier-envelope phase," in Conference on Frontiers in Optics 2005 / the 89th OSA Annual Meeting, Laser Science XXI, Oct. 2005, Tucson, Arizona, USA. 
[10] N. Ishii, C. Y. Teisset, S. Koehler, E. E. Serebryannikov, T. Fuji, T. Metzger, F. Krausz, A. Baltuška, and A. M. Zheltikov, "Widely tunable soliton frequency shifting of few-cycle laser pulses,” Phys. Rev. E 74, 036617-1-036617-10 (2006).

[11] T. Fuji, N. Ishii, C. Y. Teisset, X. Gu, Th. Metzger, A. Baltuška, N. Forget, D. Kaplan, A. Galvanauskas, and F. Krausz, "Parametric amplification of few-cycle carrierenvelope phase-stable pulses at 2.1 um," Opt. Lett. 31, 1103-1105 (2006).

[12] T. Metzger, C. Y. Teisset, and F. Krausz, "High-Repetition-Rate Picosecond Pump Laser Based on a Yb:YAG Disk Amplifier for Optical Parametric Amplification," in Conference on Advanced Solid-State Photonics, OSA Technical Digest Series (CD) (Optical Society of America, 2008), paper TuA2.

[13] X. Gu, G. Marcus, Y. Deng, T. Metzger, C. Teisset, N. Ishii, T. Fuji, A. Baltuška, R. Butkus, V. Pervak, H. Ishizuki, T. Taira, T. Kobayashi, R. Kienberger, and F. Krausz, "Generation of carrier-envelope-phase-stable 2-cycle $740-\mu \mathrm{J}$ pulses at $2.1-\mu \mathrm{m}$ carrier wavelength," Opt. Express 17, 62-69 (2009).

[14] T. Metzger, A. Schwarz, C. Y. Teisset, D. Sutter, A. Killi, R. Kienberger, and F. Krausz, "High-repetition-rate picosecond pump laser based on an Yb:YAG disk amplifier for optical parametric amplification," Opt. Lett. to be published (2009). 


\section{Acknowledgement}

Finally I would like to thank everybody helping and supporting me during the time of the above mentioned work.

First of all, I would like to thank Prof. Ferenc Krausz for giving me the opportunity to work in his group at the Faculty of Electrical Engineering at the University of Technology in Vienna and later at the Max-Planck-Institute of Quantum Optics in Garching. His absolute support and indefatigable efforts provided ideal research conditions - technically and humanly.

I would like to thank Prof. Heinz Lehr and Alexander Binder as appraiser of this work. Many thanks to Prof. Reinhard Kienberger for his complaisant support and supervision.

I appreciate the help of Catherine Yuriko Teisset sharing all the disastrous and great moments in the laboratory together with me. Thanks to the climbers Franz Tavella, Hauke Engel und Jens Rauschenberger which made a few throwbacks in the lab forgotten in the verticals in Europe. For the pleasant teamwork for the past six years I would like to thank Takao Fuji, Martin Schultze, Wolfram Helml, Alexander Schwarz, Adrian Cavalieri, Eleftherios Goulielmakis, Karl Schmid, Xun Gu, Yunpei Deng, Gilad Marcus and László Turi.

Dirk Sutter from Trumpf Laser GmbH \& Co. I would like to thank for all his richness of ideas, the time he spent in discussions with me and making the collaboration between Trumpf and the MPQ possible. I would like to thank Adolf Giesen and Friedrich Dausinger for the collaboration with the IFSW. For the great support during the time we spent in the laboratory at the IFSW I would like to thank Detlef Nickel, Daniel Kuntze, Mikhail Larionov, Christian Stoltzenburg, Aldo Antognini, Karsten Contag, Yalcin Yarimca, Roland Greschner and Jochen Speiser.

The author acknowledges the funding of this work by the German "Bundesministerium für Bildung, Wissenschaft, Forschung und Technologie" (BMBF) in the frame of the FEMTONIK research program FULMINA, project no. (FKZ): 13 N8724.

Special thanks to all my officemates during my time as a Ph.D. student in Vienna and Garching.

Many thanks to Georg Korn for pushing me to think about building a multi-joule and high repetition rate disk based laser.

Further I owe due to many reasons thank to a lot of persons, some are listed below:

Klaus Witte, Alois Böswald, Manfred Fischer, Harald Haas, Georg Brandl, Walter Fölsner, Anton Horn, Axel Raufer, Hans-Peter Schönauer, Thomas Strobl and his great team in the workshop, Monika Wild, Bettina Schütz, Chantal Arz, Barbara Podolski, Regina Fass, Alexander Apolonskiy, Roswitha Graf, Carsten Fallnich, Dietmar Kracht, Jörg Baier, Dirk Nodop, Tina Clausnitzer, Jens Limpert, Takunori Taira, ...

My parents I thank for their worldly wisdom in many respects and their benevolence in accompanying my dissertation. 


\section{Curriculum Vitae}

THOMAS METZGER

MAX-PLANCK-INSTITUT FÜR QUANTENOPTIK

HANS-KOPFERMANN-STR. 1

85748 GARCHING

GERMANY

E-MAIL: THOMAS.METZGER@MPQ.MPG.DE

TEL.: +49(89)32905-603

WWW.ATTOWORLD.DE

\section{Personal data}

\begin{tabular}{ll}
\hline Date of birth: & 18.03.1973 \\
Place of birth: & Mutlangen \\
Citizenship: & German \\
Marital status: & unmarried
\end{tabular}

\section{Education}

08/2004 - present Ph.D. student in the group of Prof. Krausz at the Max-PlanckInstitute of Quantum Optics, Garching, Germany.

Subject: High repetition rate ps-pump lasers for OPCPAs, infrared OPA pumped by Nd:YLF rod lasers and Yb:YAG thin disk regenerative chirped pulse amplifiers, Nd:YLF amplifier.

10/2002 - 07/2004 Ph.D. student in the group of Prof. Krausz at the Vienna University of Technology, Faculty of Electrical Engineering, Austria.

Subject: Design of diode end pumped ps-pump lasers, all-in-one laser for OPCPAs.

01/2001 - 02/2002 Diploma thesis in the group of Prof. Hügel at the University Stuttgart, Faculty of Strahlwerkzeuge IFSW, Germany, realized at the Laser- und Medizin-Technologie, Berlin GmbH, Berlin, Germany.

Subject: High aspect laser drilling of ceramics with different wavelength and pulse durations, heat conduction model for pulsed laser ablation of ceramics, SHG and FHG of pulsed high average power Nd:YAG lasers.

12/1998-11/1999 Research assistant in the group of Prof. Nagel at the University Stuttgart, Faculty of Biomedizinische Technik, realized at the Laserund Medizin-Technologie, Berlin GmbH, Berlin, Germany.

Subject: Time-resolved laser scattering measurement for bone density characterization.

10/1995-12/1998 Research assistant in the group of Prof. Hügel at the University Stuttgart, Faculty of Strahlwerkzeuge IFSW, Germany.

Subject: Chemical vapor deposition of diamond by $\mathrm{CO}_{2}$ laser induced plasma. Laser coating with Nd:YAG laser.

10/1992 - 02/2002 Master of Science in Mechanical Engineering (Dipl.-Ing.) at the University Stuttgart, Germany.

Main subjects: Laser technology and biomedical technique.

Abitur (high school graduation) in Schwäbisch Gmünd, Germany. 


\section{Extra-educational experience}

\begin{tabular}{|c|c|}
\hline $03 / 2002-09 / 2002$ & $\begin{array}{l}\text { Internship at Spectra-Physics in Mountainview, USA. } \\
\text { Subject: End pumped and Q-switched intracavity third harmonic } \\
\text { Nd:Vanadate laser design. }\end{array}$ \\
\hline $07 / 2001-10 / 2001$ & $\begin{array}{l}\text { Cook and kitchen help at Club der Freischwimmer in Berlin, Ger- } \\
\text { many. }\end{array}$ \\
\hline $02 / 1999-12 / 2000$ & $\begin{array}{l}\text { Research Assistant at INPRO GmbH in Berlin, Germany. } \\
\text { Subject: Laser welding and cutting of steel and aluminum with a } \\
6 \mathrm{~kW} \text { cw Nd:YAG laser. Laser tempering of steel. Transla- } \\
\text { tion of laser courses (sensor controlled laser welding) for } \\
\text { Spanish VW workers. }\end{array}$ \\
\hline $05 / 1996-10 / 1996$ & $\begin{array}{l}\text { Teaching street children in Spanish and Mathematics in Quito, Ecua- } \\
\text { dor. }\end{array}$ \\
\hline $12 / 1994-03 / 1995$ & $\begin{array}{l}\text { Internship at Sudosilo S.A. in Córdoba, Argentina. } \\
\text { Subject: Tempering of steel and hardness measurements of tempered } \\
\text { parts with different testing processes. }\end{array}$ \\
\hline $04 / 1992-05 / 1996$ & $\begin{array}{l}\text { Research Assistant at ARADEX GmbH in Lorch, Germany. } \\
\text { Subject: Repair of electronic coin changers of Mars Electronics Inc. } \\
\text { in vending machines, soldering of boards, assembling of } \\
\text { PCs and control panels for CNC machines. }\end{array}$ \\
\hline
\end{tabular}




\title{
High-repetition-rate picosecond pump laser based on a Yb:YAG disk amplifier for optical parametric amplification
}

\author{
Thomas Metzger, ${ }^{1, *}$ Alexander Schwarz, ${ }^{2}$ Catherine Yuriko Teisset, ${ }^{1}$ Dirk Sutter, ${ }^{3}$ Alexander Killi, ${ }^{3}$ \\ Reinhard Kienberger, ${ }^{2,4}$ and Ferenc Krausz ${ }^{1,2}$ \\ ${ }^{1}$ Department of Physics, Ludwig-Maximilians-Universität München, Am Coulombwall 1, 85748 Garching, Germany \\ ${ }^{2}$ Max-Planck-Institute of Quantum Optics, Hans-Kopfermann-Strasse 1, 85748 Garching, Germany \\ ${ }^{3}$ TRUMPF-Laser GmbH + Co. KG, Aichhalder Straße 39, 78713 Schramberg, Germany \\ ${ }^{4}$ Department of Physics, Technische Universität München, James Franck Straße, 85748 Garching, Germany \\ *Corresponding author: thomas.metzger@mpq.mpg.de \\ Received April 23, 2009; accepted May 26, 2009; \\ posted June 8, 2009 (Doc. ID 110518); published July 7, 2009 \\ We report an optically synchronized picosecond pump laser for optical parametric amplifiers based on an \\ $\mathrm{Yb}$ :YAG thin-disk amplifier. At $3 \mathrm{kHz}$ repetition rate, pulse energies of $25 \mathrm{~mJ}$ with $1.6 \mathrm{ps}$ pulse duration \\ were achieved with an rms fluctuation in pulse energy of $<0.7 \%$ by utilizing a broadly intermittent single- \\ energy regime in the deterministic chaos of a continuously pumped regenerative amplifier. (c) 2009 Optical \\ Society of America \\ OCIS codes: $140.3280,140.7090,140.1540,140.3480$.
}

Producing isolated attosecond extreme-UV pulses via high harmonic generation requires high-energy fewcycle pulses preferably with kilohertz repetition rates for exploring hyperfast electronic phenomena [1]. Ultrashort pulses in the range of a few femtoseconds at approximately kilohertz repetition rates have been demonstrated using a complex design consisting of an oscillator, a multipass chirped pulse amplifier, and an additional nonlinear compression stage $[2,3]$. However, it has been difficult to extend these systems to the multimillijoule level [4]. Optical parametric chirped pulse amplifiers (OPCPAs) have emerged as a powerful alternative for creating broadband fewcycle pulses and are the only method by which highenergy multimillijoule few-cycle coherent light pulses have been generated $[5,6]$. However, current OPCPA designs suffer from complex stretcher and compressor elements. Stretching the seed pulse to a significant fraction of the pump pulse duration is required for efficient energy extraction, but extensive stretching to tens of picoseconds requires highly dispersive prisms or grating components and subsequently intricate adaptive dispersion management schemes for proper recompression [7,8]. The use of shorter pump pulses in the range of a few picoseconds would eliminate the need for such a large stretching and compression ratio and allow stretching of the seed pulses by passing through a few-centimeters-long dispersive optical material and recompression by a highly efficient compressor made up of a few chirped multilayer mirrors [9]. Furthermore, the threshold intensity for optical damage of transparent materials increases even faster than the $1 / \tau^{1 / 2}$ for laser pulse durations decreasing below 20 ps [10]. As a consequence, exposing the nonlinear crystal to higher intensities allows the same optical parametric amplification (OPA) gain to be attained with a shorter crystal and hence over a broader bandwidth [11]. Recent experiments in the area of IR multikilohertz few picosecond amplifiers without chirped pulse amplification (CPA) [12] showed temporal pulse broadening owing to selfphase-modulation (SPM) for energies below $1 \mathrm{~mJ}$ [13]. Fortunately, in the near-IR spectral range, CPA can be implemented with low loss owing to the availability of gratings with diffraction efficiencies of $\sim 99 \%$ [14].

In this Letter, we present an Yb:YAG picosecond pump laser operated within a broadly intermittent stable single-energy regime in the deterministic chaos of a continuously diode-laser-pumped regenerative amplifier that produces $1.6 \mathrm{ps}, 25 \mathrm{~mJ}$ pulses at a repetition rate of $3 \mathrm{kHz}$. Chaotic behavior and bifurcations in a regenerative amplifier were first theoretically described and partially experimentally analyzed in [15]. But only in [16] was it theoretically discovered that intermittent stable regimes could be used for creating a stable multikilohertz regenerative amplifier with small energy fluctuations. In this work the laser has been designed to especially use intermittent stable regimes for creating high-energy pulses for use in pumping an efficient, high gain, high contrast, IR OPCPA with a simplified stretchercompressor system. The repetition rate of the regenerative amplifier was matched to that of our $3 \mathrm{kHz}$ Ti:sapphire-based OPA seed source but can be tuned up to $f_{r}=20 \mathrm{kHz}$. The experimental apparatus, schematically shown in Fig. 1, consists of a seed laser, a stretcher, a fiber preamplifier, a pulse picker, an amplifier resonator, and a compressor. The seed pulses are delivered by an ultrabroadband chirped-mirror Ti:sapphire oscillator (Rainbow, Femtolasers Produktions $\mathrm{GmbH}$ ) covering the spectral range of $650-1100$ $\mathrm{nm}$ [17]. This approach allows for nearly jitter-free optical synchronization between pump and signal pulses in the OPCPA, as they are both derived from the same source [8]. The resultant seed energy in the 


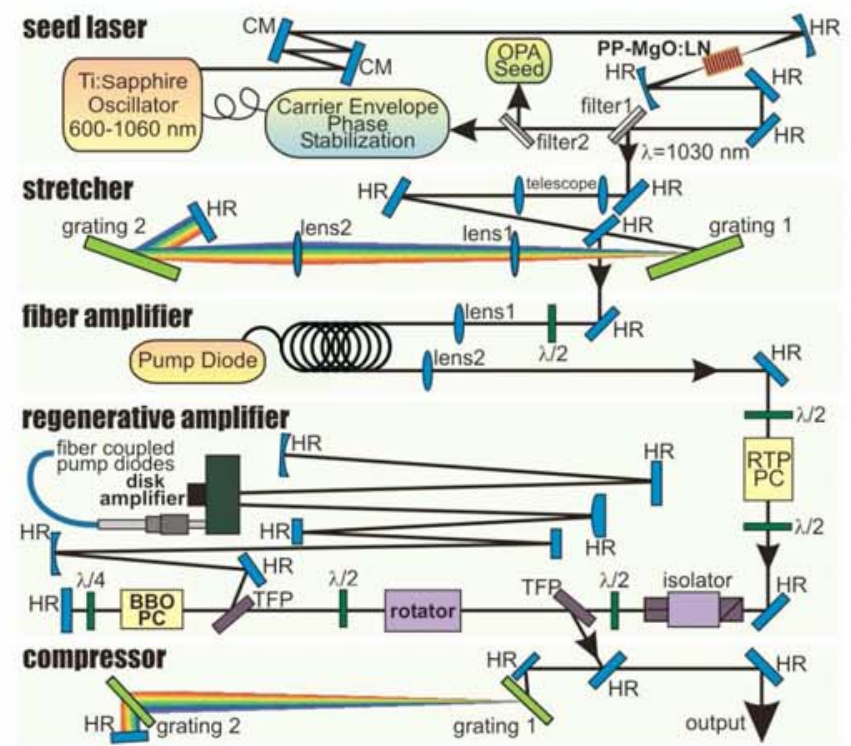

Fig. 1. (Color online) Schematical laser setup. HR, high reflecting mirror; CM, chirped mirror; RTP, rubidium titanyl phosphate; PC, Pockels cell; TFP, thin-film polarizer; $\lambda / 2$, half-wave-plate; $\lambda / 4$, quarter-wave-plate; PP$\mathrm{MgO}: \mathrm{LN}, \mathrm{MgO}$-doped periodically poled $\mathrm{LiNbO}_{3}$ crystal.

spectral region of the $\sim 5.3 \mathrm{~nm}$ broad gain bandwidth of $\mathrm{Yb}$ :YAG, centered at $1030 \mathrm{~nm}$, is $\sim 2 \mathrm{pJ}$. Before amplification, the seed pulse is temporally dispersed in a $4 f$ stretcher consisting of a pair of reflection gratings with 1740 lines $/ \mathrm{mm}$, inserted at an angle of incidence of $59^{\circ}$, and two lenses $(f=1000 \mathrm{~mm})$ [18]. In a double pass the stretcher introduces a group delay dispersion of $1.19 \times 10^{8} \mathrm{fs}^{2}$ and has $70 \%$ throughput efficiency. The stretcher supports a $4 \mathrm{~nm}$ broad spectrum centered at $1030 \mathrm{~nm}$ and cuts off all other spectral components owing to the limited size of the used optical components. The calculated Fourier-limited pulse duration of the $4 \mathrm{~nm}$ broad seed spectrum supported by the stretcher is $\sim 0.77 \mathrm{ps}$ (FWHM). After stretching to $\sim 430 \mathrm{ps}$, a $40 \mathrm{~dB}$ homebuilt doublestage fiber amplifier from Friedrich-SchillerUniversity Jena increases the total seed energy to $\sim 1.2 \mathrm{~nJ}$, and a rubidium titanyl phosphate (RTP) Pockels cell is used to slice pulses from the $\sim 70 \mathrm{MHz}$ oscillator pulse train at a frequency of $6 \mathrm{kHz}$. An isolator protects the Pockels cell against feedback from the amplifier. To avoid nonlinear effects, a Pockels cell using a relatively short $(20 \mathrm{~mm}) \beta$-barium borate (BBO) crystal (quarter-wave voltage of $16 \mathrm{kV}$ ) has been chosen for switching the selected pulses in and out of the amplifier cavity. The $1 / e^{2}$ beam diameter in this element is $2.8 \mathrm{~mm}$, leading to a calculated $B$ integral of 1.67. Inside the cavity, pulses are amplified in an arrangement similar to that described in [19]. The amplifier head from TRUMPF Laser contains a $\sim 1 / 10 \mathrm{~mm}$ thin, $12.5 \%$ doped, Yb:YAG disk mounted on a heat sink, and is pumped in a 20 pass cavity with up to $500 \mathrm{~W}$ at $940 \mathrm{~nm}$ by laser diodes (LDM 500 , Laserline $\mathrm{GmbH}$ ) in a region of $2.8 \mathrm{~mm}$ in diameter. Following amplification, recompression is performed using two transmission gratings with 1400 lines/mm arranged in a double-pass Littrow configu- ration $\left(\mathrm{GDD}=-1.19 \times 10^{8} \mathrm{fs}^{2}\right)$, with overall transmission of $\sim 77 \%$.

Operating in an intermittent stable regime in the deterministic chaos of the continuously pumped regenerative amplifier, we reproducibly achieve an average output power of $75 \mathrm{~W}$ at $f_{r}=3 \mathrm{kHz}$ with pulse energies exceeding $25 \mathrm{~mJ}$, a pulse-to-pulse stability of $<0.7 \%$ (rms), a pulse duration of $1.6 \mathrm{ps}$ and a bandwidth of $1 \mathrm{~nm}$ (FWHM) at a center wavelength of $1030.2 \mathrm{~nm}$. Figure 2 shows the measured autocorrelation $(\mathrm{AC})$ trace and the optical spectrum of the laser output at a pulse energy of $25 \mathrm{~mJ}$. The resulting time-bandwidth product of 0.46 is within $5 \%$ of the transform limit of 0.441 for Gaussian pulses. Gain narrowing reduces the bandwidth to $1 \mathrm{~nm}$ and shortens the pulse duration inside the amplifier cavity to 200 ps before compression.

Figure 3 shows the energy distribution in a bifurcation diagram acquired by incrementing the pump power in steps of $2 \mathrm{~W}$ over the range of $P_{p}=100-300 \mathrm{~W}$ with the number of round trips in the amplifier being kept constant at $N_{\mathrm{rt}}=150$. Owing to low thermal lensing in the disk, the amplifier cavity and the output beam profile were stable and did not change over this entire pump power range. For each set value of the pump power, 200 amplified pulses extracted with the Pockels cell from the cavity were recorded by a fast, energy-calibrated, photodiode (rise time $<175 \mathrm{ps}$ ). Figure 3 plots the range of pulse energies attained this way for each individual pump power. For $P_{p}<140 \mathrm{~W}$ normal amplification at the full $6 \mathrm{kHz}$ repetition rate of the Pockels cell is observed. For $P_{p}>140 \mathrm{~W}$ the first bifurcation occurs, leading to completely chaotic behavior above $170 \mathrm{~W}$. A stable regime of period doubling commences for $P_{p}>240 \mathrm{~W}$, resulting in a repetition rate of $3 \mathrm{kHz}$ of the amplified pulses.

The energy of the intermediate pulses extracted from the cavity amounts to $\sim 0.2 \%$ of that of the main amplified pulses. For pumping an OPCPA this is negligible owing to the exponential scaling of nonsaturated OPA gain with the pump intensity [11]. At $3 \mathrm{kHz}$ repetition rate the maximum output power of $75 \mathrm{~W}$ after the compression is reached at a pump power of $284 \mathrm{~W}$. The total optical efficiency was cal-

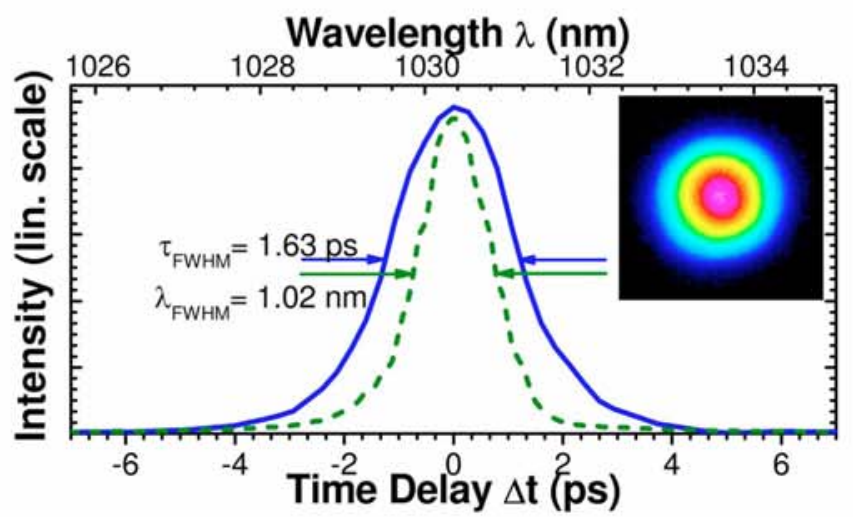

Fig. 2. (Color online) AC trace, optical spectrum, and beam profile (measured $M^{2}<1.1$ ) of the laser output. The $\mathrm{AC}$ trace corresponds to a FWHM pulse duration of $1.6 \mathrm{ps}$, assuming a Gaussian pulse shape. 


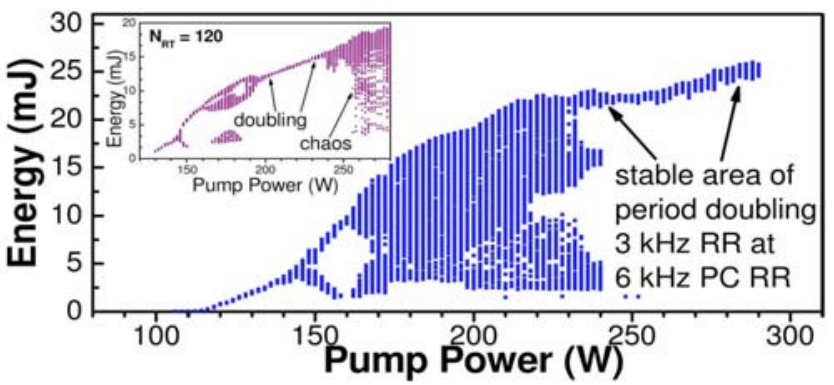

Fig. 3. (Color online) Measured bifurcation diagram for 150 round trips in dependence of the pump power at a repetition rate of $6 \mathrm{kHz}$. The inset shows the result of the same measurement with 120 round trips.

culated to be $26 \%$, including compression losses. Complete saturation of the gain medium in every second amplification cycle ensures highly stable operation, with pulse-to-pulse energy fluctuations lower than $0.7 \%$ rms. By varying parameters such as the number of round trips and the pump power, which affect the laser's bifurcation characteristics $[15,16]$, the intermittent stable pulse regime of period doubling can be shifted to different pulse-energy regimes, allowing output energies at a repetition rate of $3 \mathrm{kHz}$ between 2 and $25 \mathrm{~mJ}$. For example, by lowering the number of round trips in the amplifier, the intermittent stable regime is followed by a further chaotic interval and stable output energy occurs between a lower and upper pump power limit for period doubling; see the inset of Fig. 3. The disk amplifier also exhibits an excellent beam quality, which is also critical for parametric amplification. The amplifier cavity supports only the $\mathrm{TEM}_{00}$ fundamental laser mode, resulting in a near-diffraction-limited beam with $M^{2}<1.1$ measured at the maximum pulse energy. The measured beam profile is shown as an inset in Fig. 2.

In conclusion, we have reported the generation of $25 \mathrm{~mJ}, 1.6 \mathrm{ps}$ pulses delivered in a near-diffractionlimited beam at a wavelength of $1030.2 \mathrm{~nm}$ with a rms pulse-to-pulse stability of smaller than $0.7 \%$ and a repetition rate of $3 \mathrm{kHz}$ from a regenerative chirped pulse amplifier based on a thin-disk Yb:YAG gain medium. The peak power exceeds $15 \mathrm{GW}$. To the best of our knowledge, this is the most powerful multikilohertz regenerative amplifier reported to date.

We acknowledge assistance from TRUMPF Laser. R. Kienberger acknowledges funding from the Sofja Kovalevskaja Award of the A. v. Humboldt Foundation, the European Research Council Starting Grant, and the Deutsche Forschungsgemeinschaft (DFG) Cluster of Excellence: Munich Centre for Advanced Photonics. The German Federal Ministry of Education and Research (BMBF) funded this work, project $13 \mathrm{~N} 8724$.

\section{References}

1. E. Goulielmakis, M. Schultze, M. Hofstetter, V. S. Yakovlev, J. Gagnon, M. Uiberacker, A. L. Aquila, E. M. Gullikson, D. T. Attwood, R. Kienberger, F. Krausz, and U. Kleineberg, Science 320, 1614 (2008).

2. S. Sartania, Z. Cheng, M. Lenzner, G. Tempea, Ch. Spielmann, F. Krausz, and K. Ferencz, Opt. Lett. 22, 1562 (1997).

3. M. Nisoli, S. De Silvestri, O. Svelto, R. Szipöcs, K. Ferencz, Ch. Spielmann, S. Sartania, and F. Krausz, Opt. Lett. 22, 522 (1997).

4. S. Backus, R. Bartels, S. Thompson, R. Dollinger, H. C. Kapteyn, and M. M. Murnane, Opt. Lett. 26, 465 (2001).

5. F. Tavella, A. Marcinkevičius, and F. Krausz, Opt. Express 14, 12822 (2006).

6. S. Witte, R. Th. Zinkstok, A. L. Wolf, W. Hogervorst, W. Ubachs, and K. S. E. Eikema, Opt. Express 14, 8168 (2006).

7. F. Tavella, Y. Nomura, L. Veisz, V. Pervak, A. Marcinkevičius, and F. Krausz, Opt. Lett. 32, 2227 (2007).

8. N. Ishii, C. Y. Teisset, T. Fuji, S. Köhler, K. Schmid, L. Veisz, A. Baltuška, and F. Krausz, IEEE Sel. Top. Quantum Electron. 12, 173 (2006).

9. R. Szipocs, K. Ferencz, C. Spielmann, and F. Krausz, Opt. Lett. 19, 201 (1994).

10. B. C. Stuart, M. D. Feit, S. Herman, A. M. Rubenchik, B. W. Shore, and M. D. Perry, Phys. Rev. B 53, 1749 (1996).

11. G. Cerullo and S. De Silvestri, Rev. Sci. Instrum. 74, 1 (2003).

12. D. Strickland and G. Mourou, Opt. Commun. 56, 219 (1985).

13. A. Giesen and J. Speiser, IEEE J. Sel. Top. Quantum Electron. 13, 598 (2007).

14. N. Destouches, A. Tishchenko, J. Pommier, S. Reynaud, O. Parriaux, S. Tonchev, and M. Ahmed, Opt. Express 13, 3230 (2005).

15. J. Dörring, A. Killi, U. Morgner, A. Lang, M. Lederer, and D. Kopf, Opt. Express 12, 1759 (2004).

16. M. Grishin, V. Gulbinas, and A. Michailovas, Opt. Express 15, 9434 (2007).

17. T. Fuji, A. Unterhuber, V. S. Yakovlev, G. Tempea, A. Stingl, F. Krausz, and W. Drexler, Appl. Phys. B 77, 125 (2003).

18. O. E. Martinez, IEEE J. Quantum Electron. 23, 59 (1987).

19. C. Hönninger, I. Johannsen, M. Moser, G. Zhang, A. Giesen, and U. Keller, Appl. Phys. B 65, 423 (1997). 